\title{
International \\ Nuclear Fuel Cycle Fact Book
}

\author{
K. M. Harmon \\ L. T. Lakey \\ I. W. Leigh
}

Issued November 1980

Latest Revision March 1984

Prepared for the U.S. Department of Energy under Contract DE-AC06-76RLO 1830

Pacific Northwest Laboratory

Operated for the U.S. Department of Energy by Battelle Memorial Institute 


\title{
DISCLAIMER
}

This report was prepared as an account of work sponsored by an agency of the United States Government. Neither the United States Government nor any agency thereof, nor any of their employees, makes any warranty, express or implied, or assumes any legal liability or responsibility for the accuracy, completeness, or usefulness of any information, apparatus, product, or process disclosed, or represents that its use would not infringe privately owned rights. Reference herein to any specific commercial product, process, or service by trade name, trademark, manufacturer, or otherwise, does not necessarily constitute or imply its endorsement, recommendation, or favoring by the United States Government or any agency thereof. The views and opinions of authors expressed herein do not necessarily state or reflect those of the United States Government or any agency thereof.

\author{
PACIFIC NORTHWEST LABORATORY \\ operated by \\ BATTELLE \\ for the \\ UNITED STATES DEPARTMENT OF ENERGY \\ under Contract DE-AC06-76RLO 1830
}

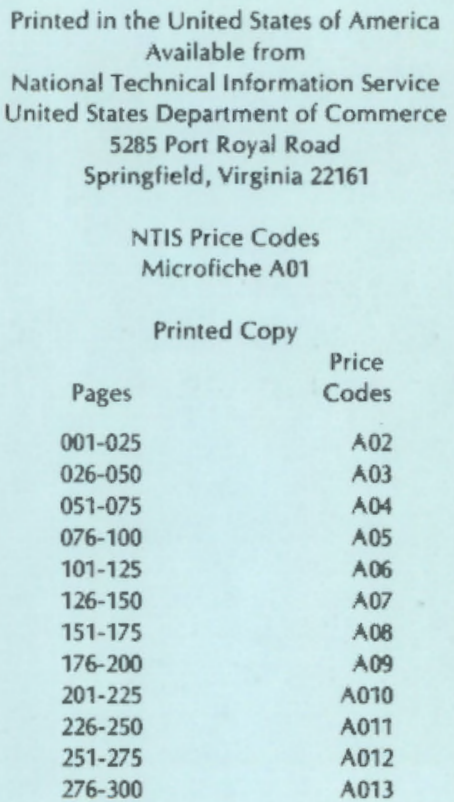




\section{5}

PNL-3594 Rev. 4 UC -70

INTERNATIONAL NUCLEAR

FUEL CYCLE FACT BOOK
K. M. Harmon
L. T. Lakey
I. W. Leigh

Issued November 1980

Latest Revision March 1984

Prepared for

the U.S. Department of Energy

under Contract DE-AC06-76RL0 1830

Pacific Northwest Laboratory

Richland, Washington 99352 


\section{PREFACE}

As the US Department of Energy (DOE) and DOE contractors have become inore and more involved in cooperative activities with other nations in nuclear fuel cycle and waste management activities, a need has developed for a ready source of information concerning foreign fuel cycle programs, facilities, and personnel. This Fact Book was compiled to meet that need.

The information contained herein has been obtained from many unclassified sources: nuclear trade journals and newsletters; reports of foreign visits and visitors; CEC, IAEA, and OECD/NEA activities reports; proceedings of conferences and workshops; etc.

The information in the fact Book is subject to frequent change. If you have suggestions which would improve the usefulness of the book or uncover more current information, let us know so that these changes can be included in periodic updates.

Manager, International Program Support Office Nuclear Waste Technology Program Office Pacific Northwest Laboratory

P.0. Box 999

Richland, WA 99352

Telephone/FTS: (509) $375-2697$ or $375-2758$

Telex: $\quad 15-2874$ 
Introduction and 0verview. . . ....... Intro-1 National Summaries

Argentina................... AR -

Australia................... AS-1

Austria............... AU-1

Belgium. . . . . . . . . . . . . . BE-1

Brazil .................... BR -1

Canada ............... . . CA-1

China (People's Repub1ic of)......... $\mathrm{CH}-1$

Denmark. . . . . . . . . . . . DA-1

Finland. .................. FI-1

France ............... . . FR -1

Germany (Federal Republic) .......... GW-1

India. . . . . . . . . . . . . . IN-1

Italy.................. IT-1

Japan. ................ JA-1

Korea (Republic of Korea)............ KS-1

Mexico................ MX

Netherlands............. NE - 1

Pakistan ................ PK 1

South Africa.............. . SF -1

Spain.................. SP -1

Sweden ................. SW-1

Switzerland. . . . . .......... SZ-1

Tajwan (Republic of China)......... . . TA-1

Union of Soviet Socialist Republics. . . . . UR-1

United Kingdom ............... UK-1

International Agencies

CMEA (Council for Mutual Economic Assistance). . Int 1-1

EC (European Communities). . . . . . . . . Int $1-2$

CEC (Commission of the European Communities). . Int 1-2

CEC Joint Research Centre.......... Int 1-3

JRC-Ispra Establishment........... Int $1-3$

JRC-Karlsruhe Establishment......... Intl-4

IAEA (International Atomic Energy Agency).... Intl-5

ICRP (International Commission on Radiological

Protection)............. Int $1-7$

OECD Nuclear Energy Agency (NEA) ....... Int 1-8

Energy Supply and Demand......... Energy-1

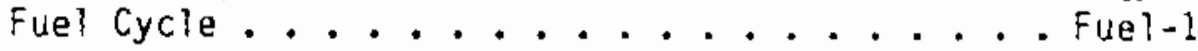


TABLE OF CONTENTS (contd)

Page

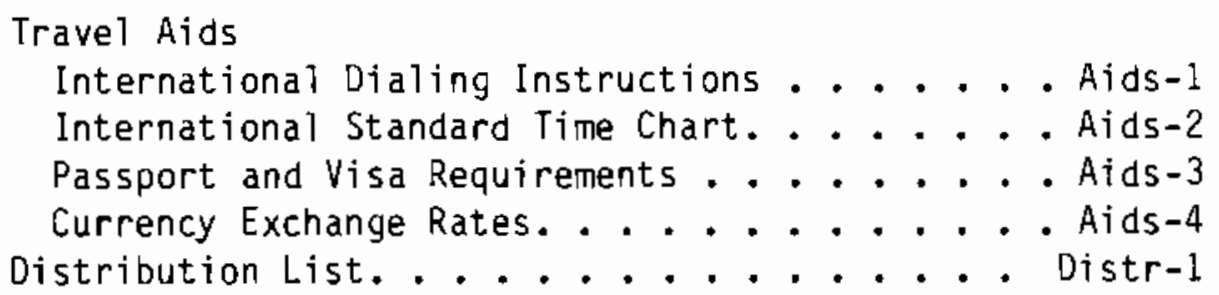




\section{ACRONYMS}

ORGANIZATIONS AND FACILITIES

\begin{tabular}{|c|c|}
\hline AAEC & Australian Atomic Energy Commission \\
\hline AEB & Atomic Energy Bureau (Korea) \\
\hline AEC & Atomic Energy Commission (Korea) \\
\hline AEC & Atomic Energy Council (Taiwan) \\
\hline AECB & Atomic Energy Control Board (Canada) \\
\hline AECL & Atomic Energy of Canada, Limited \\
\hline AERB & Atomic Energy Regulation Board (India) \\
\hline ANDRA & $\begin{array}{l}\text { Agence Nationale pour la Gestion des } \\
\text { Déchets Radioactifs (France) }\end{array}$ \\
\hline AWRE & Atomic Weapons Research Establishment (UK) \\
\hline BARC & Bhabha Atomic Energy Centre (India) \\
\hline BGR & $\begin{array}{l}\text { Bundesanstalt für Geowissenschaften und } \\
\text { Rohstoffe (FRG) }\end{array}$ \\
\hline BMFT & $\begin{array}{l}\text { Bundesministerium für Forschung und } \\
\text { Technologie (FRG) }\end{array}$ \\
\hline BMI & Bundesministerium des Innern (FRG) \\
\hline BNFL & British Nuclear Fuels Limited \\
\hline BRE & Building Research Establishment (UK) \\
\hline BRGM & $\begin{array}{l}\text { Bureau de Recherches Géologiques et } \\
\text { Minières(France) }\end{array}$ \\
\hline CANMET & $\begin{array}{l}\text { Canada Centre for Metals and Energy } \\
\text { Technology }\end{array}$ \\
\hline CDTN & $\begin{array}{l}\text { Centro de Desenvolvimento de Technologia } \\
\text { Nuclear de Nuclebràs (Brazil) }\end{array}$ \\
\hline CEA & $\begin{array}{l}\text { Commissariat à l'Énergie Atomique } \\
\text { (France) }\end{array}$ \\
\hline CEC & Commission of the European Communities \\
\hline CEGB & Central Electricity Generating Board (UK) \\
\hline CEN/SCK & $\begin{array}{l}\text { Centre d'Études de l'Énergie Nucledire/ } \\
\text { Studiecentrum voor Kernenerqíe } \\
\text { (Belgium) }\end{array}$ \\
\hline$C E N-C A$ & Centre d'Études Nucléaires de Cadarache \\
\hline CEN-F aR & $\begin{array}{l}\text { Centre d'Études Nucléaires de } \\
\text { Fontenay-aux-Roses (France) }\end{array}$ \\
\hline CEN-G & $\begin{array}{l}\text { Centre d'Études Nucléaires de Grenoble } \\
\text { (France) }\end{array}$ \\
\hline CEN-S & Centre d'Études Nucléaires de Saclay \\
\hline CFC & COGEMA Framatone Combustible (France) \\
\hline CMEA & Council for Mutual Economic Assistance \\
\hline CNEA & $\begin{array}{l}\text { Comisión Nacional de Energía Atomica } \\
\quad \text { (Argentina) }\end{array}$ \\
\hline
\end{tabular}




\begin{tabular}{|c|c|}
\hline CNEN & $\begin{array}{l}\text { Commissao Nacional de Energía Nuciear } \\
\text { (Brazil) }\end{array}$ \\
\hline CNSNS & $\begin{array}{l}\text { Comisión Nacional de Seguridad Nuclear y } \\
\text { Salvaguardias (Mexico) }\end{array}$ \\
\hline COGEMA & $\begin{array}{l}\text { Compagnie Générale des Matières } \\
\text { Nucléaires (France) }\end{array}$ \\
\hline CPE & Countries with Centrally Planned Economies \\
\hline CRIEPI & $\begin{array}{l}\text { Central Research Institute of Electric } \\
\text { Power Industry (Japan) }\end{array}$ \\
\hline CRPPH & $\begin{array}{l}\text { Committee on Radiation Protection and } \\
\text { Public Health (NEA) }\end{array}$ \\
\hline $\operatorname{CSN}$ & Consejo de Seguridad Nuclear (Spain) \\
\hline DAM & Direction Des Applications Militaires \\
\hline DBE & $\begin{array}{l}\text { Deutsche Gesellschaft zum Bau und Betrieb } \\
\text { von Endlagern für Abfallstoffe mbH } \\
\text { (FRG) }\end{array}$ \\
\hline DHI & Deutsches Hydrographisches Institut (FRG) \\
\hline DOE & Department of the Environment (UK) \\
\hline DWK & $\begin{array}{l}\text { Deutsche Gesellschaft für Wiederaufar- } \\
\text { beitung von Kernbrennstoffen mbH (FRG) }\end{array}$ \\
\hline EC & European Communities \\
\hline ECN & Energy Centrum Nederland \\
\hline EIR & $\begin{array}{l}\text { Eidgenössisches Institut für Reaktor } \\
\text { Forschung (Switzerland) }\end{array}$ \\
\hline EMR & Energy, Mines and Resources (Canada) \\
\hline ENEA & $\begin{array}{l}\text { Energia Nucleare e Delle Energie } \\
\text { Alternative (Italy) }\end{array}$ \\
\hline ENEL & $\begin{array}{l}\text { Ente Nazionale per l'Energia Elettrica } \\
\text { (Italy) }\end{array}$ \\
\hline ENI & Ente Nazionale Idrocarburi (Italy) \\
\hline ENUSA & Empresa Nacional del Uranio S.A. (Spain) \\
\hline EPB & Electric Power Bureau (Korea) \\
\hline ESCOM & $\begin{array}{l}\text { Electricity Supply Commission (South } \\
\text { Africa) }\end{array}$ \\
\hline FBFC & $\begin{array}{l}\text { Société Franco-Belge de Fabrication de } \\
\text { Combustibles (France) }\end{array}$ \\
\hline GIRIO & $\begin{array}{l}\text { Government Industrial Research Institute- } \\
\text { Osaka (Japan) }\end{array}$ \\
\hline GSF / IfT & $\begin{array}{l}\text { Gesellschaft für Strahlen-und- } \\
\text { Umweltforschung } \mathrm{mbH} \text { / Institut fur } \\
\text { Tieflagerung (FRG) }\end{array}$ \\
\hline HMI & Hahn-Meitner Institut (FRG) \\
\hline IAEA & International Atomic Energy Agency \\
\hline ICRP & $\begin{array}{l}\text { International Commission on Radiological } \\
\text { Protection }\end{array}$ \\
\hline IGS & Institute of Geological Sciences (UK) \\
\hline
\end{tabular}




\begin{tabular}{|c|c|}
\hline INER & $\begin{array}{l}\text { Institute of Nuclear Energy Research } \\
\text { (Taiwan) }\end{array}$ \\
\hline ININ & $\begin{array}{l}\text { Instituto Nacional de Investigaciones } \\
\text { Nucleares (Mexico) }\end{array}$ \\
\hline IPEN & $\begin{array}{l}\text { Instituto de Pesquisas Energéticas e } \\
\text { Nucleares (Brazil) }\end{array}$ \\
\hline IPSN & $\begin{array}{l}\text { Institut de Protection et de Sûreté } \\
\text { Nucléaire(France) }\end{array}$ \\
\hline IRD I & $\begin{array}{l}\text { Institut de Recherche Technologie et de } \\
\text { Developpement Industriel }\end{array}$ \\
\hline JAER I & Japan Atomic Energy Research Institute \\
\hline JEN & Junta de Energía Nuclear (Spain) \\
\hline $\mathrm{JRC}$ & Joint Research Center (CEC) \\
\hline KAERI & Korea Advanced Energy Research Institute \\
\hline KBS & $\begin{array}{l}\text { Kärnkräftavfallets Behandling och } \\
\text { Slutförvaring(Sweden) }\end{array}$ \\
\hline KEMA & $\begin{array}{l}\text { N.V. Tot Keuring van Electrotechnische } \\
\text { Materialen Arnhem (Netherlands) }\end{array}$ \\
\hline KEPKO & Korea Electric Power Corporation \\
\hline KEWA & $\begin{array}{l}\text { Kernbrennst off-Wiederaufarbeitungs - GmbH } \\
\quad(F R G)\end{array}$ \\
\hline KFA & Kernforschungsanlage Jülich GmbH (FRG) \\
\hline KfK & $\begin{array}{l}\text { Kernforschungszentrum Karlsruhe GmbH } \\
\text { (FRG) }\end{array}$ \\
\hline KOPEL & Korea Power Engineering Company \\
\hline KTM & Royal Institute of Technology \\
\hline KWU & Kraftwerk Union $A G$ (FRG) \\
\hline LDC & Less Developed Country \\
\hline MAFF & $\begin{array}{l}\text { Ministry of Agriculture, Fisheries and } \\
\text { Food (UK) }\end{array}$ \\
\hline MITI & $\begin{array}{l}\text { Ministry of International Trade and } \\
\text { Industry }\end{array}$ \\
\hline NAGRA/CEDRA & $\begin{array}{l}\text { Nationalen Genossenschaft für de Lagerung } \\
\text { Radioaktiver Abfälle (Switzerland) }\end{array}$ \\
\hline NAK & $\begin{array}{l}\text { Naennden foer Hantering av Atvaenr } \\
\text { Kernsbrensle (Sweden) }\end{array}$ \\
\hline NEA & Nuclear Energy Agency (OECD) \\
\hline NI I & Nuclear Installations Inspectorate (UK) \\
\hline NIREX & $\begin{array}{l}\text { Nuclear Industry Radioactive Waste } \\
\text { Executive (UK) }\end{array}$ \\
\hline NRPB & $\begin{array}{l}\text { National Radiological Protection Board } \\
\text { (UK) }\end{array}$ \\
\hline NSC & Nuclear Safety Commission (Japan) \\
\hline NTL & $\begin{array}{l}\text { Nuclear Transport Limited (UK, France } \\
\text { and FRli) }\end{array}$ \\
\hline
\end{tabular}




\begin{tabular}{|c|c|}
\hline OECD & $\begin{array}{l}\text { Organization for Economic Cooperation } \\
\text { and Development }\end{array}$ \\
\hline ÖFZS & $\begin{array}{l}\text { Österreichisches Forschungszentrum } \\
\text { Seibersdorf GmbH (Austria) }\end{array}$ \\
\hline ONDRAF & $\begin{array}{l}\text { Organisme National de Déchets Radioactifs } \\
\text { et des Matières Fissiles (Belgium) }\end{array}$ \\
\hline PNC & $\begin{array}{l}\text { Power Reactor and Nuclear Fuel Deveiopment } \\
\text { Corporation (Japan) }\end{array}$ \\
\hline PTB & $\begin{array}{l}\text { Physikalisch-Technische Bundesanstalt } \\
\text { (FRG) }\end{array}$ \\
\hline RWC & $\begin{array}{l}\text { Radioactive Waste Management Center } \\
\text { (Japan) }\end{array}$ \\
\hline RWMAC & $\begin{array}{l}\text { Radioactive Waste Management Advisory } \\
\text { Committee (UK) }\end{array}$ \\
\hline RWMC & $\begin{array}{l}\text { Radioactive Waste Management Committee } \\
\text { (NEA) }\end{array}$ \\
\hline SGN & $\begin{array}{l}\text { Société Générale pour des Techniques } \\
\text { Nouvelles (France) }\end{array}$ \\
\hline SKI & Statens Kärnkraftinspektion (Sweden) \\
\hline SRD & Safety and Reliability Directorate (UK) \\
\hline STA & Science and Technology Agency (Japan) \\
\hline TN & Transnucleaire (France) \\
\hline UKAEA & United Kingdom Atomic Energy Authority \\
\hline UKOR/UCOR & $\begin{array}{l}\text { Uranium Enrichment Corporation (South } \\
\text { Africa) }\end{array}$ \\
\hline UNIREP Or URG & $\begin{array}{l}\text { United Reprocessors GmbH (FRG, France, } \\
\text { UK consortium) }\end{array}$ \\
\hline URAMEX & Uranio Mexicano \\
\hline URENCO & $\begin{array}{l}\text { British-German-Dutch uranium enrichment } \\
\text { consortium }\end{array}$ \\
\hline WAK & $\begin{array}{l}\text { Wiederaufarbeitungsanlage Karlsruhe } \\
\text { Betriebsgesselschaft mbH (FRG) }\end{array}$ \\
\hline WOCA & $\begin{array}{l}\text { World Outside Centrally Planned } \\
\text { Economies Areas }\end{array}$ \\
\hline
\end{tabular}

TECHNICAL

$\begin{array}{ll}\text { AFR } & \begin{array}{l}\text { Away-from-reactor spent fuel storage } \\ \text { facility }\end{array} \\ \text { AGR } & \text { Advanced gas-cooled reactor, } \mathrm{UO}_{2} \text { fuel } \\ (U K) & \text { FRG acid digestion plant to be built at } \\ \text { MLONA } & \text { Advanced thermal reactor }\end{array}$


AVB

AVH

AVM

BLWR

BWR

CANDU

D\&D

ESTER

FBR

FBTR

FIPS

FRP

GCHWR

GCR

GWd

GWe

HAO

HARVEST

HL.LW

HLW

HTGR

HTR

HWLWR

HWR

IFTF

ILW

$\mathrm{KgHM}$

$\mathrm{KgU}$

LGR

LHGW

LLW

LLLW

LMFBR

LWCHW

LWR

MLW

MOX
AVM-type HLW vitrification plant to be built at Eurochemic (Belgium)

AVM-type waste vitrification plant to be built at La Hague (France)

Atelier de vitrification de Marcoule (France)

Boiling light water reactor

Boiling water reactor

Canada deuterium uranium reactor

Decontamination and decommissioning

Multistage pot calcination-vitrification process for HLW (Italy)

Fast breeder reactor

Fast breeder test reactor

Fission product solidification process (FRG/KFA)

Fuel reprocessing plant

Gas-cooled, heavy water moderated reactor Gas-cooled, graphite moderated reactor Gigawatt days

$10^{9}$ watts of electricity (1000 MWe)

Oxide head-end

British waste vitrification process

High-level liquid waste

High-level waste

High-temperature, gas-cooled reactor

High-temperature reactor

Heavy water moderated, light water cooled (same as LWCHW)

Heavy water reactor

Immobilized Fuel Test Facility (Canada)

Intermediate-level waste

Kilograms heavy metal

Kilograms uranium

Light water cooled, graphite moderated reactor

Low heat generating waste

Low-level waste

Low-level liquid waste

Liquid metal fast breeder reactor

Light water cooled, heavy water moderated (same as HWLWR)

Light water reactor

Medium-level waste (same as intermediatelevel)

Mixed (plutonium/uranium) oxide 


$\begin{array}{ll}\text { Mtce } & \text { Million metric tons of coal equivalent } \\ \text { Mtoe } & \text { Million metric tons of oil equivalent } \\ \text { MTR } & \text { Materials test reactor } \\ \text { MW } & \text { Megawatts } \\ \text { MWd/t } & \text { Megawatt days per tonne } \\ \text { MWe } & \text { Megawatts electric } \\ \text { MWt } & \text { Megawatts thermal } \\ \text { NPT } & \text { Non-Proliferation Treaty } \\ \text { PAMELA } & \text { West German vitrification pilot plant } \\ \text { PFR } & \text { (Mol, Belgium) } \\ \text { PHWR } & \text { Prototype Fast Reactor (UK) } \\ \text { PLWR } & \text { Pressurized heavy water reactor } \\ \text { PURR } & \text { Pressurized light water reactor } \\ & \text { Prototype d'usine de retraitement des } \\ \text { PWR } & \text { rapids (France) } \\ \text { QUAD } & \text { Pressurized water reactor } \\ \text { t/a } & \text { 10ls Btu } \\ \text { tHM } & \text { Tons per annum } \\ \text { tSWU/a } & \text { Tons heavy metal } \\ \text { tU } & \text { Tons of separative work (uranium } \\ \text { TEK0 } & \text { Tonrichnent) per annum } \\ \text { THORP } & \text { Nons uranium per annum } \\ \text { THTR } & \text { Theradioactive semi-works facility (FRG) } \\ \text { Th/U } & \text { Thorium high-temperature reactor } \\ \text { TRU } & \text { Thorium/Uranium } \\ \text { TWh } & \text { Transuranic } \\ \text { URL } & \text { Terawatt hour (million megawatt hours) } \\ \text { WIP } & \text { Wasterground Research Laboratory (Canada) } \\ & \end{array}$




\section{INTRODUCTION AND OVERVIEW}




\section{INTRODUCTION}

The International Fuel Cycle Fact Book has been compiled in an effort to provide 1) an overview of worldwide nuclear power and fuel cycle programs and 2) current data concerning fuel cycle and waste management facilities, R\&D programs, and key personnel in countries other than the United States. Additional information on each country's program is available in the International Source Book: Nuclear Fuel cycle Research and Development, PNL-2478, Rev. 2.

The Fact Book is organized as follows:

1. Overview section--summary tables which indicate national involvement in nuclear reactor, fuel cycle, and waste management development activities.

2. National summaries--a section for each country which summarizes nuclear policy, describes organizational relationships and provides addresses, names of key personnel, and facilities information.

3. International agencies-a section for each of the international agencies which has significant fuel cycle involvement.

4. Energy supply and demand--summary tables, including nuclear power projections.

5. Fuet cycle--summary tables.

6. Travel aids--international dialing instructions, international standard time chart, passport and visa requirements, and currency exchange rate. 
TABLE 1. Reactor Mix

No. of Reactors

On1ine, Ordered, or

Under Construction (12-31-83) Reactor

Country LWR HWR GCR Other

Argentina

3

Belgium 7

Brazil 3

Bulgaria 4

Canada 24

China (People's 1

Republic)

Czechoslovakia 10

Egypt 2

Finland 4

France 52

Germany (FRG) 26

Germany (GDR) 7

Hungary 4

India

It aly

Japan

Korea (South)

Mexico

Netherlands 2

Pakistan

Philippines 1

Poland

Rumania

South Africa

Spain

Sweden

2

2

4

39

81

2

2

Switzerland

Taiwan

USSR

UX

USA

140

2 FBR, 22 LGR

1 FBR

FBR

1 HTR, 1 LGR,

1 FBR

Yugoslavia

1 
TABLE 2. Fuel Production Program(a)

\begin{tabular}{|c|c|c|c|c|c|}
\hline \multirow[b]{2}{*}{ Country } & \multirow{2}{*}{$\begin{array}{l}\text { Mining(b) } \\
\text { \& Milling }\end{array}$} & \multirow{2}{*}{$\begin{array}{l}\text { Conver- } \\
\text { sion }\end{array}$} & \multirow{2}{*}{$\begin{array}{l}\text { Enrich- } \\
\text { ment }\end{array}$} & \multicolumn{2}{|c|}{$\begin{array}{c}\text { Fuel } \\
\text { Fabrication }\end{array}$} \\
\hline & & & & & \\
\hline Argentina & $x$ & $x$ & $R \& 0$ & $x$ & $R \& D$ \\
\hline Australia & $x$ & & R\&D & & \\
\hline Belgi um & & $x$ & (c) & $x$ & $x$ \\
\hline Brazil & $x$ & $x$ & $x$ & $x$ & \\
\hline Canada & $x$ & $x$ & & $x$ & \\
\hline France & $x$ & $x$ & $x$ & $x$ & $x$ \\
\hline Germany (FRG) & $x$ & $x$ & $x$ & $x$ & $x$ \\
\hline India & $x$ & $x$ & & $x$ & $x$ \\
\hline Italy & $x$ & $x$ & (c) & $x$ & $x$ \\
\hline Japan & $x$ & $x$ & $x$ & $x$ & $x$ \\
\hline Korea (South) & $R \& D$ & R\&D & & $R \& D$ & \\
\hline Mexico & $x$ & $x$ & & $x$ & \\
\hline Netherl ands & & & $x$ & & \\
\hline South Africa & $x$ & $x$ & $x$ & & \\
\hline Spain & $x$ & & & $x$ & \\
\hline Sweden & & & (c) & $x$ & \\
\hline USSR & $x$ & $x$ & $x$ & $x$ & $x$ \\
\hline UK & & $x$ & $x$ & $x$ & $x$ \\
\hline USA & $x$ & $x$ & $x$ & $x$ & $x$ \\
\hline Yugoslavia & $x$ & & & & \\
\hline
\end{tabular}

(a) Legend: $x$ - industrial plant in operation or on order R\&D - R\&D stages only

(b) Commercial mining and milling operations are also conducted in the following countries: Central African Republic, Gabon, German Democratic Republic, Namibia, Niger, Philippines and Portugal.

(c) Partnership in Eurodif enrichment plant in France. 
TABLE 3. Spent Fue1 Management and Waste Treatment Programs

\begin{tabular}{|c|c|c|c|c|}
\hline \multirow[b]{2}{*}{ Country } & \multirow{2}{*}{$\begin{array}{c}\text { Spent } \\
\text { Fuel } \\
\text { Transport } \\
\end{array}$} & \multirow{2}{*}{$\begin{array}{c}\text { Fuel Repro- } \\
\text { cessing }\end{array}$} & \multicolumn{2}{|c|}{$\begin{array}{c}\text { Waste } \\
\text { Conditioning }\end{array}$} \\
\hline & & & HLW & Non-HLW \\
\hline Argentina & & $R \& D$ & R\&D & $x$ \\
\hline Australia & & & $R \& D$ & \\
\hline Austria & & & $R \& D$ & $\mathrm{R} \& \mathrm{D}$ \\
\hline Belgium & & $x$ & $x$ & $x$ \\
\hline Brazil & & $R \& D$ & $R \& D$ & $R \& D$ \\
\hline Canada & & R\&D & $R \& D$ & $x$ \\
\hline Denmark & & & & $R \& D$ \\
\hline Finland & & & & $x$ \\
\hline France & $x$ & $x$ & $x$ & $x$ \\
\hline Germany (FRG) & $x$ & $x$ & $x$ & $x$ \\
\hline India & & $x$ & $x$ & $x$ \\
\hline Italy & & $x$ & R\&D & $x$ \\
\hline Japan & $x$ & $x$ & $x$ & $x$ \\
\hline Mexico & & & & $x$ \\
\hline Nether lands & & & & $x$ \\
\hline Spain & & & & $x$ \\
\hline Sweden & $x$ & & $R \& D$ & $x$ \\
\hline Switzerland & & & & $x$ \\
\hline USSR & $x$ & $x$ & $R \& D$ & $x$ \\
\hline UK & $x$ & $x$ & $x$ & $x$ \\
\hline USA & $x$ & $x$ & $x$ & $x$ \\
\hline
\end{tabular}


IABLE 4. Spent Fuel and Waste Disposal R\&D Programs

\begin{tabular}{|c|c|c|c|c|}
\hline Country & $\begin{array}{c}\text { Spent } \\
\text { Fuel } \\
\text { Disposal } \\
\end{array}$ & $\begin{array}{c}\text { HLW } \\
\text { Disposal }\end{array}$ & $\begin{array}{l}\text { LLW/ILW } \\
\text { Disposal }\end{array}$ & $\begin{array}{c}\text { Spent } \\
\text { Fuel } \\
\text { Storage }\end{array}$ \\
\hline Argentina & & $x$ & $x$ & $x$ \\
\hline Austria & & $x$ & & \\
\hline Belgium & & $x$ & $x$ & \\
\hline Brazil & & & $x$ & \\
\hline Canada & $x$ & $x$ & $x$ & $x$ \\
\hline Denmark & $x$ & $x$ & & \\
\hline Finland & $x$ & $x$ & $x$ & $x$ \\
\hline France & & $x$ & $x$ & $x$ \\
\hline Germany (FRG) & $x$ & $x$ & $x$ & \\
\hline Germany (DGR) & & & $x$ & \\
\hline India & & $x$ & $x$ & \\
\hline Italy & & $x$ & $x$ & $x$ \\
\hline Japan & & $x$ & $x$ & $x$ \\
\hline Mexico & & & $x$ & $x$ \\
\hline Netherlands & & $x$ & $x$ & $x$ \\
\hline South Africa & $x$ & $x$ & $x$ & $x$ \\
\hline Spain & $x$ & $x$ & $x$ & $x$ \\
\hline Sweden & $x$ & $x$ & $x$ & $x$ \\
\hline Switzerland & $x$ & $x$ & $x$ & $x$ \\
\hline USSR & & $x$ & $x$ & $x$ \\
\hline UK & & $x$ & $x$ & $x$ \\
\hline USA & & $x$ & $x$ & $x$ \\
\hline
\end{tabular}


.

• 
NATIONAL SUMMARIES 
ARGENTINA 
ARGENTINE HOLIDAYS

*Asterisks denote movable holidays

January 1

January 6

*February

*March/April

*March/April

May 1

May 25

*June

June 20

July 9

August 15

August 17

October 12

November 1

November 6

December 8

December 25
New Year's Day

Epiphany

Carnival

Holy Thursday

Good Friday

Labor Day

Anniv. of 1810 Revolution

Corpus Christi Day

Flag Day

Independence Day

Assumption Day

Death of Gen. San Martin

Columbus Day

All Saints' Day

Bank Holiday

Immaculate Conception

Christmas

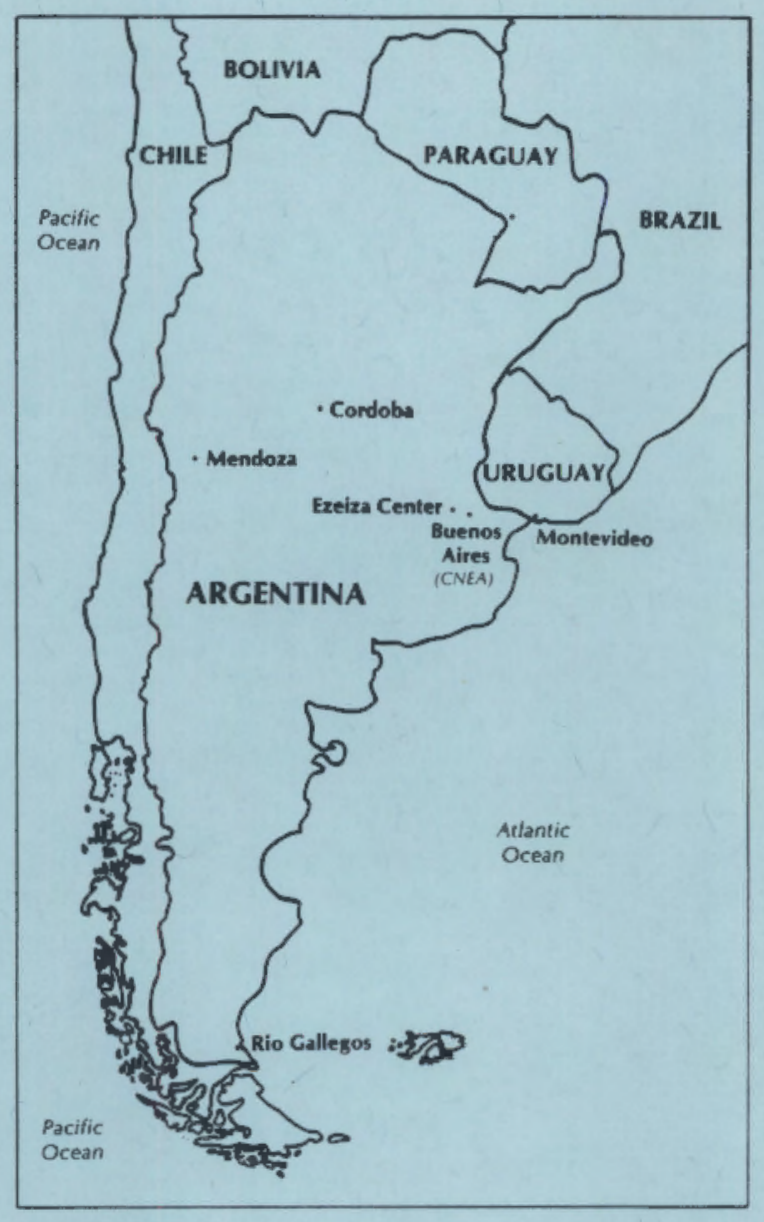




\section{ARGENTINA}

ENERGY

$\begin{array}{lcc}\begin{array}{l}\text { Population } \\ \text { Energy Demand }\end{array} & 1980 & 27 \text { million } \\ \text { Electric Power Plant Capacity } & 1979 & 40 \text { Mtoe } \\ \text { Electric Power Production } & 1982 & 12 \text { GWe } \\ & & 27 \text { TWh-- } \\ 8 \% \text { nuclear } & >50 \% \text { fossil } \\ & & \text { Balance hydro }\end{array}$

NUCLEAR POWER

National Policy. High priority on self-sufficient CANDUbased nuclear power industry; government ownership and operation of all nuclear power plants; develop nuclear plant and services export capability.

Nuclear Power Plant Capacity $1980 \quad 0.3$ GWe

$1985 \quad 0.9$ GWe

$1990 \quad 1.6$ GWe

$2000 \quad 3.0$ GWe

Reactor Mix

1982 HWR: 1 (1974);

$2(1983-88)$

INDUSTRIAL FUEL CYCLE

Policy. Develop domestic capability for $\mathrm{D}_{2} \mathrm{O}$ production and al1 phases of the CANDU-type PHWR fuel cycle; may export Pu to breeder nations.

Waste Management Strategy. Build repository in granite formation, use it for interim (dry) storage of spent fuels until ready to dispose of $H L W$.

$\begin{array}{rrr}\text { Cumulative Spent Fuel } & 1980 & 416 \mathrm{tU} \\ \text { Arisings (HWR) } & 1985 & 940 \mathrm{tU} \\ & 1990 & 1,900 \mathrm{tU} \\ & 2000 & 5,800 \mathrm{tU}\end{array}$


$\underline{\text { Industrial-Scale Activities }}$

1. $D_{20}$ production: $1984--200 \mathrm{t} / \mathrm{a} \mathrm{D}_{2} 0$ enrichment plant, supplied by a Swiss firm; develop domestic technology.

2. Uranium mining and milling ( $t / a)$ : 1981--260; $1985--680$.

3. Conversion of yellowcake to $\mathrm{UO}_{2}$ (tU/a): 1983--150.

4. Uranium enrichment: $500 \mathrm{~kg} / \mathrm{a}$ of $20 \%$ enriched U-- 1985 .

5. Fuel fabrication: $\cup 0_{2}$ fuels - see Ezeiza Atomic Centre; MOX fuel R\&D.

6. Fuel reprocessing: R\&D (see Ezeiza Atomic Centre).

INTERNATIONAL RELATIONSHIPS

1. Member of IAEA but opposed to NPT; has signed but not ratified Tlatalclo Treaty.

2. Nuclear plants and technology purchased from Canada, $F R G$, and Switzerland.

3. Technical cooperation with Latin America countries, primarily Brazil and Peru.

ORGANI ZATION

The Comision Nacional de Energía Atomica (CNEA, National Atomic Energy Commission), owns and operates all facilities.

\section{CNEA}

Comision Nacional de Energía Atomica (CNEA)

Avenida del Libertador 8250

1429 Buenos Aires

Argentina

Tel: (54) 1707711

Telex: 121388

President (Interim)

Renato Radi Chela 


\section{EZEIZA ATOMIC CENTRE}

Location. 10 miles northwest of Buenos Aires, near airport.

\section{Facilities}

1. Conversion of yellowcake to $\mathrm{UO}_{2}(150 \mathrm{tU} / \mathrm{a})$.

2. Fuel production: the first of three planned fabrication lines started up in 1982; capacity, 1-1/2 PHWR assembly per day.

3. Fuel reprocessing: Ezeiza pilot plant, planned capacity of $20 \mathrm{kgU} /$ day feed, $10-15 \mathrm{kgPu} / \mathrm{a}$ product; cold runs--1985; hot runs--1986. Potential--expansion of pilot plant to commercial facility by early 1990 s. 



\section{AUSTRALIA}


AUSTRALIAN HOLIDAYS

*Asterisks denote movable holidays

January 1

January 29

*March/April

New Year's Day

*March/April

April 25

December 25

December 26

Australia Day

Good Friday

Easter Monday

ANZAC Day

Christmas

Boxing Day

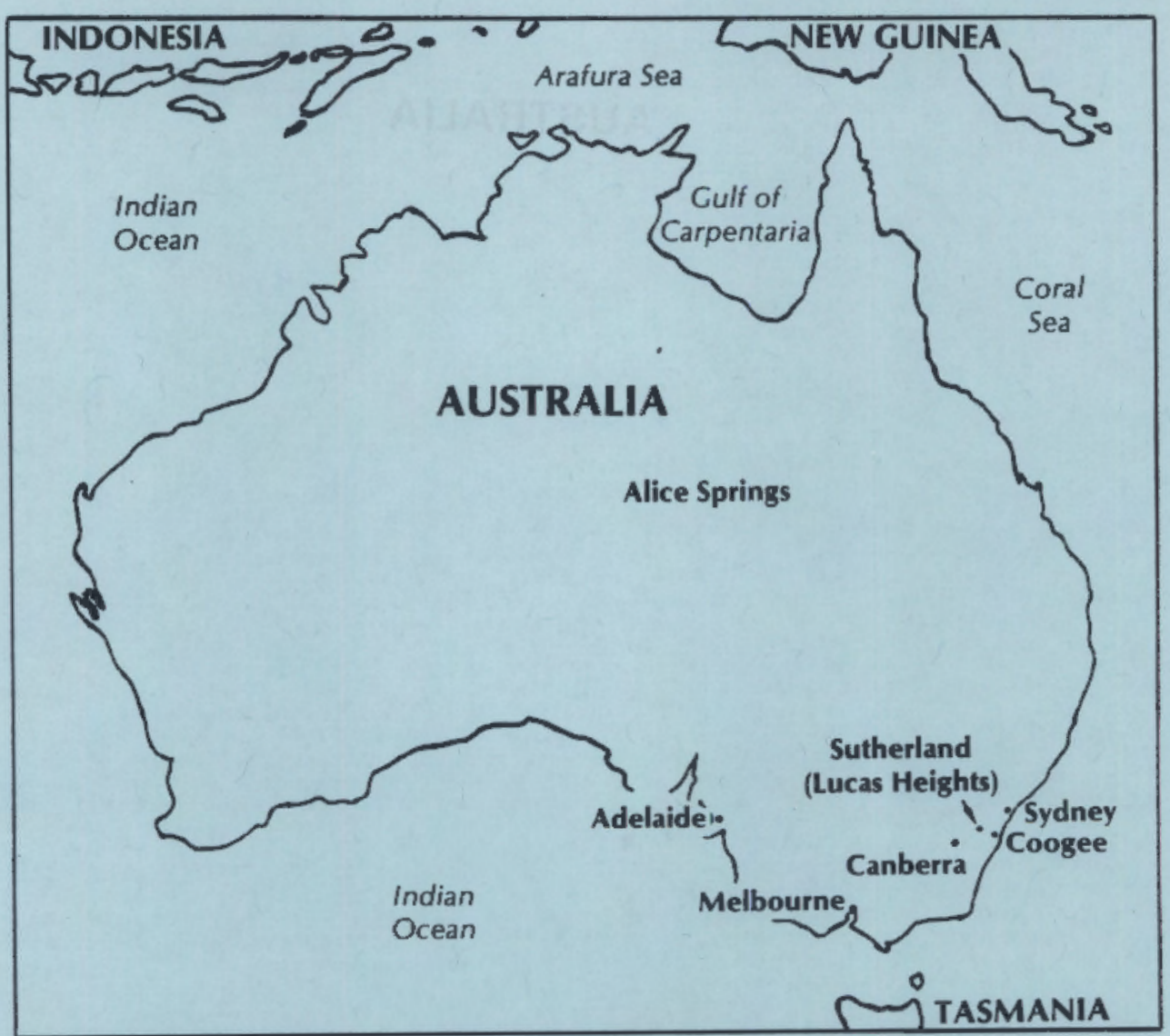




\section{AUSTRALIA}

ENERGY

Population

198215 million

Energy Resources: production exceeds consumption by about $20 \%$, but Australia imports $20 \%$ of its oil

Electric Power Production

1979

$$
\begin{aligned}
& 90.9 \text { TWh-- } \\
& 74 \% \text { solid fuels } \\
& 15 \% \text { hydro } \\
& 6 \% \text { oil } \\
& 5 \% \text { gas }
\end{aligned}
$$

NUCLEAR POWER POLICY

1. No nuclear power installed; none planned.

2. Large uranium reserves; uranium currently produced for export, but labor government opposes opening new mines and recently terminated discussions with URENCO of a proposed joint enrichment project.

3. Government sponsors nuclear R\&D, including development of:

- uranium enrichment technology

- HTGR and LMFBR systems

- SYNROC process for immobilizing HLW.

ORGANIZATION

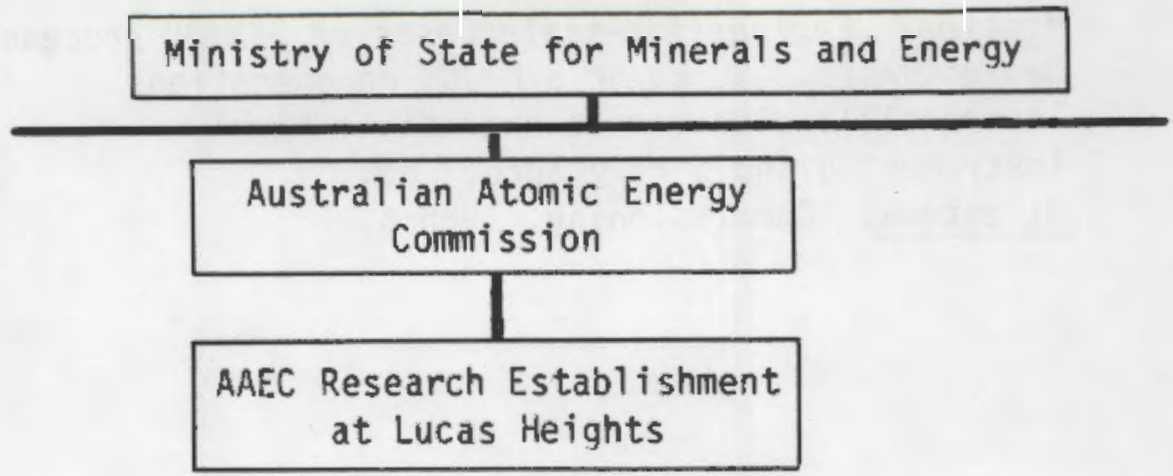




\section{AAEC LUCAS HEIGHTS RESEARCH LABORATORIES}

Australian Atomic Energy Commission Lucas Heights Research Laboratories New Illawarra Road, Lucas Heights Private Mail Bag, Sutherland, 2232 New South Wales, Australia

Tel: (61) 25430111

Chairman Telex: AA 24562

Director Deputy Director, Research

Prof. D. W. George Prof. S. T. Butler

Chemical Technology Division Materials Division Dr. J. N. Gregory Dr. P. G. Alfredsen Environmental Science Division Dr. P. M. Kelly Dr. D. R. Davy Nuclear Fuel Cycle Dr. C. J. Hardy Chemical Engineering Section Dr. D. M. Levins

Mission. Conduct nuclear R\&D; support university research and training projects in all branches of nuclear science and engineering.

Sponsors. AAEC and 17 universities.

Fuel Cycle R\&D. Uranium enrichment, HLW immobilization (SYNROC process development and waste form properties), mill tailings treatment (actinide transport, surface hydrology, and radionuclide release).

\section{Facilities}

1. Nonradioactive SYNROC Fabrication Plant

Mission. Engineering-scale tests of SYNRDC process. Design Basis. $10 \mathrm{~kg} / \mathrm{hr}$ SYNROC; no operations incompatible with remote operation; highly instrumented and partly automated. Milestone. Commissioning, 1985/6. 
Australia

AAEC LUCAS HEIGHTS RESEARCH LABORATORIES (contd)

2. SYNROC Glove Box Line

Mission. Produce SYNROC containing actinides and ${ }^{99} \mathrm{TC}$. Process Scale. Hundreds of grams. Milestone. Startup, 1984.

Hot-Cell Processing Line for SYNROC

Milestone. Startup, 1984.

AUSTRALIAN ATOMIC ENERGY COMMISSION (AAEC)

Australian Atomic Energy Commission (AAEC)

P.0 Box 41

Coogee 2034

New South Wales

Te1: (61) 26651211

Australia

Telex: AA 20273

General Manager

Maxwell Brennan

AUSTRALIAN NATIONAL UNIVERSITY

Australian National University

P.0. Box 4

Canberra 2600

Australia

Director, Research School of Prof. A. E. Ringwood Earth Sciences

Fuel Cycle R\&D. HLW immobilization (SYNROC process). 


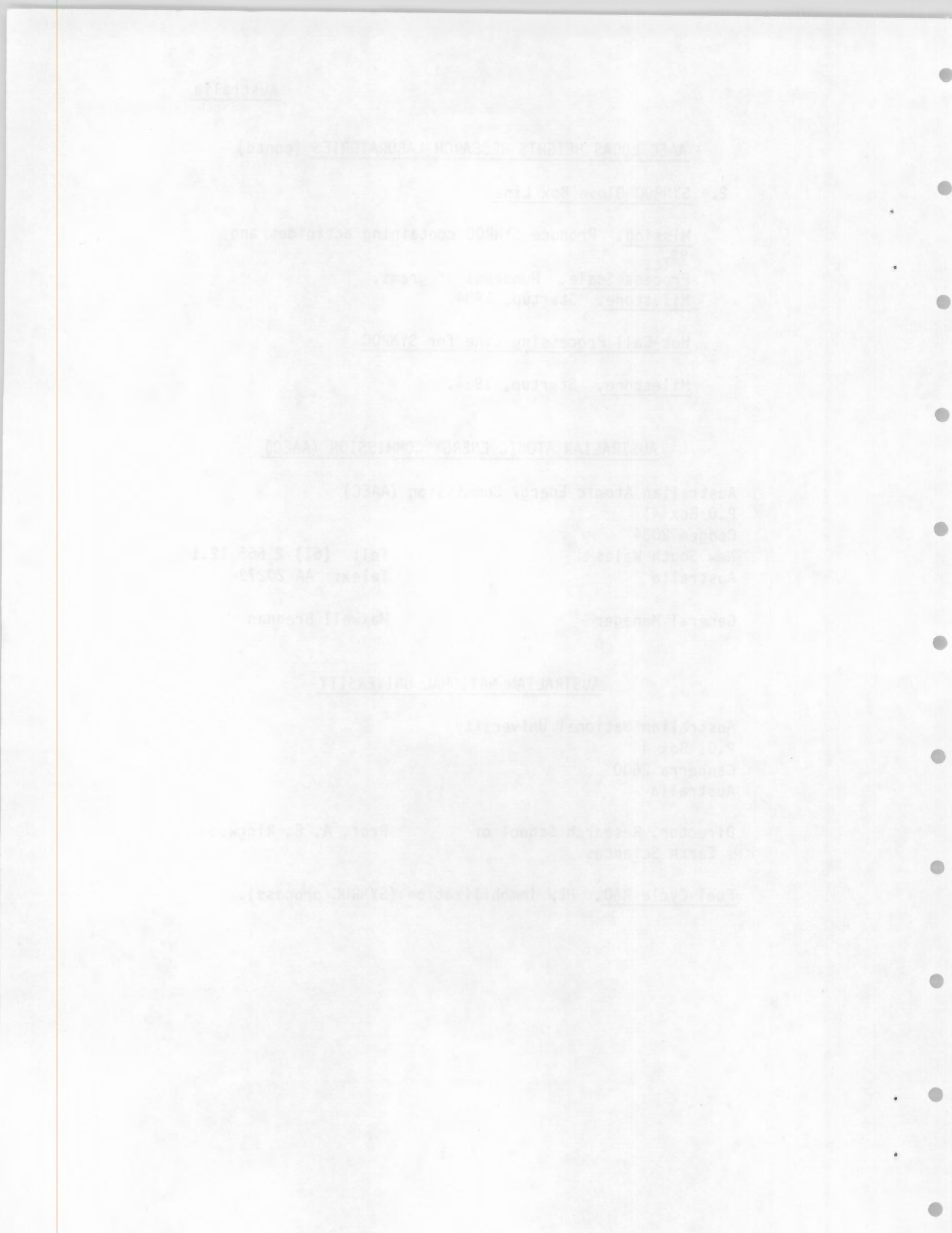




\section{AUSTRIA}




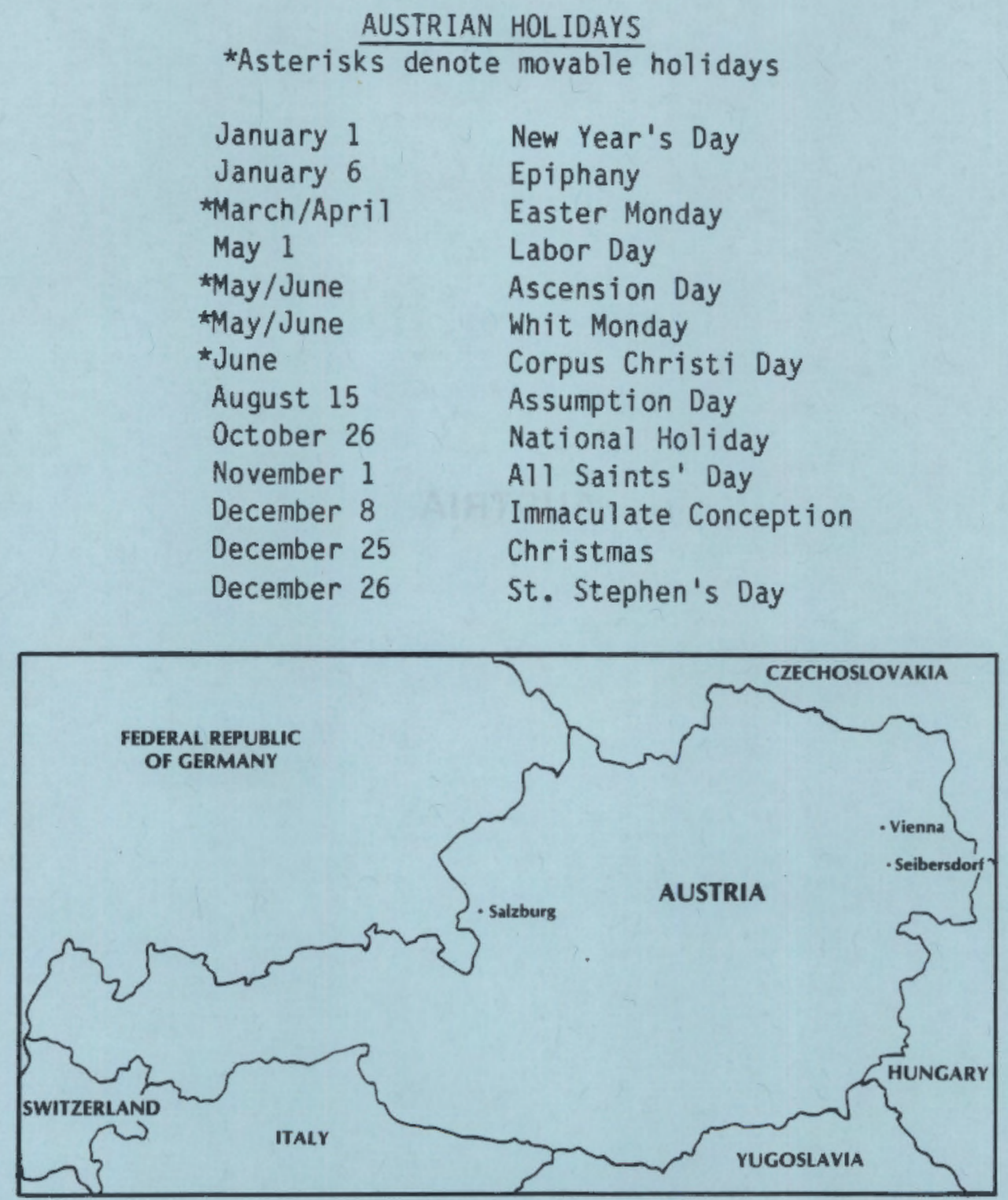


AUSTRIA

ENERGY

$\begin{array}{lcl}\begin{array}{l}\text { Population } \\ \text { Energy Demand }\end{array} & 1982 & 7.6 \text { million } \\ \text { Oil Demand } & 1979 & \begin{array}{c}27 \text { Mtoe } \\ (50-60 \% \text { imported })\end{array} \\ \text { Electric Power Plant Capacity } & 1980 & 13 \text { GWe } \\ \text { Electric Power Production } & 1980 & \begin{array}{l}12.7 \text { Mtoe } \\ (88 \% \text { imported })\end{array} \\ & & \begin{array}{l}40.6 \text { TWh-- } \\ 73 \% \text { hydro/geo. } \\ \end{array} \\ & & \begin{array}{l}12 \% \text { oil } \\ 9 \% \text { gas } \\ 6 \% \text { solid fuels }\end{array}\end{array}$

NUCLEAR POWER POLICY

1. 0.7 GWe nuclear plant at Tulnerfeld completed but not approved for operation; exercise of nuclear option requires change in Austrian law.

2. Government sponsors R\&D on reactor safety, waste treatment and waste isolation.

INDUSTRIAL FUEL CYCLE

Policy. Initial--depend on foreign suppliers. Reprocessing contract with COGEMA canceled in 1979.

Waste Management Strategy. Prepare to take back HLW canisters from foreign reprocessor, in case the nuclear option becomes acceptable. The government has joined with Egypt in studies of potential cooperative waste storage in Egypt.

ORGANIZATION

Waste treatment and disposal R\&D are the responsibility of the Austrian Research Centre Seibersdorf LTD (ÜFZS), which is headquartered in Vienna and has a laboratory at Seibersdorf. 
Austria

\section{GEOLOGICAL SURVEY}

Geological Survey of Austria

Rasumof skygasse 23

A-1031 Wien

Austria

\section{ÖFZS (AUSTRIAN RESEARCH CENTER SEIBERSDORF)}

Österreichisches Forschungszentrum

Seibersdorf GmbH

Lenaugasse 10

A-1082 Vienna

Austria

Tel: (43) 222427511

Telex: $07-5400$

A-2444 Seibersdorf

Tel: (43) $225480-0$

Austria

Telex: 014-353

Waste Management

Dr. Peter Krejsa

(43) 2254802541

Incineration Materia1

Dr. Peter Patek

Test, Cementation

Bituminization

(43) 2254802568

Dr. Karl Knotik

(43) 2254802405

Mission. R\&D in peaceful applications of nuclear energy and radiation.

Sponsors. National government, industry, and electric utilities.

Waste Management R\&D. HLW immobilization, LLW and ILW treatment, waste isolation in granite formations (geology, hydrology, heat transfer, corrosion, barrier materials, and risk analysis). 


\section{BELGIUM}


BELGIAN HOLIDAYS

*Asterisks denote movable holidays

January 1

*March/April

May 1

*May/June

*May/June

July 21

August 15

November 1

November 11

December 25
New Year's Day

Easter Monday

Labor Day

Ascension Day

Whit Monday

National Day

Assumption Day

All Saints' Day

Armistice Day

Christmas

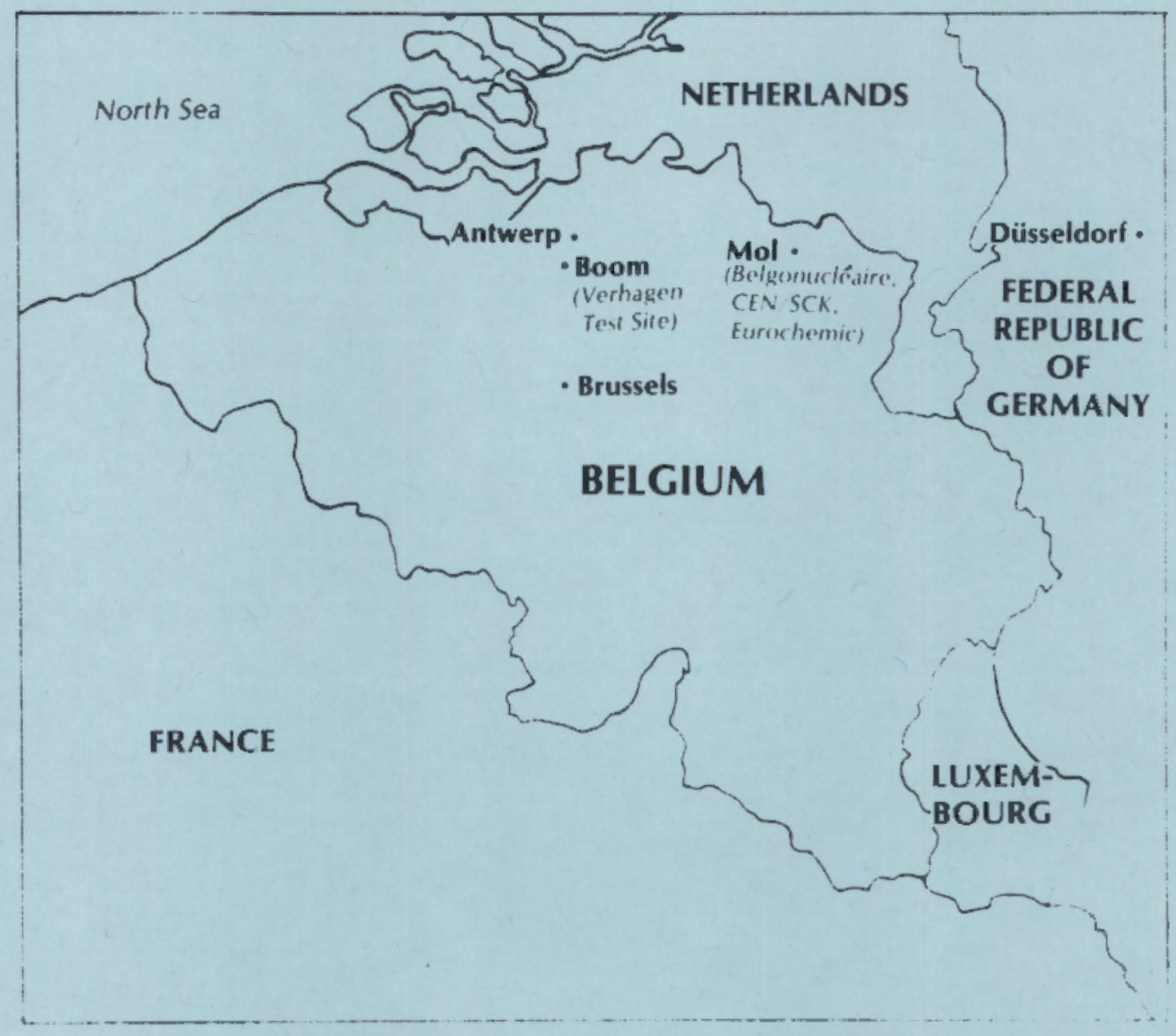




\section{BELGIUM}

ENERGY

Population

1982

9.9 million

Energy Demand

1980

49.5 Mtoe

(84\% imported)

$0 i 1$ Demand

1980

25.8 Mtoe

(100\% imported)

Electric Power Plant Capacity $1982 \quad 15$ GWe--

$23 \%$ nuclear

Electric Power Production

1982

48 TWh--

$30.2 \%$ nuclear

$1.5 \%$ hydro/geo.

$1985>50 \%$ nuclear

NUCLEAR POWER

National Policy. Complete the LWRs currently under construction; evaluate addition of an eighth (1300 MWe) unit.

Nuclear Power Plant Capacity

$\begin{array}{ll}1983 & 3.5 \text { GWe } \\ 1985 & 5.4 \text { GWe } \\ 1995 & 6.7 \text { GWe } \\ 2000 & 8.0 \text { GWe }\end{array}$

Reactor Mix

1982 PWR: $4(1975-82)$

$3(1983-85)$

Reactor Development

Participation in FRG

Kalkar SNR-300 FBR project

INDUSTRIAL FUEL CYCLE

Policy. Well-rounded capability--uranium enrichment (share in Eurodif); MOX and $\mathrm{UO}_{2}$ fuel fabrication; purchase of foreign reprocessing services; and operation of former Eurochemic plant and waste treatment facilities. 
Waste Management Strategy. Vitrify HLW in AVM-type plant and store for 50 years in surface facility; treat and immobilize other wastes; sea-dump LLW drums and dispose of HLW in clay-formation repository.

Cumulative Spent Fuel

$\begin{array}{rr}1980 & 196 \mathrm{tU} \\ 1985 & 560 \mathrm{tU} \\ 1990 & 1,290 \mathrm{tU} \\ 2000 & 3,000 \mathrm{tU}\end{array}$

Major Milestones

- Underground research laboratory

- Industrial repository (ILW, TRU)

Mid-1990s

- Startup of German HLW vitrification 1986 pilot plant (PAMELA) at Mol

- Vitrification plant (AVM Process)

- Decision on startup of renovated Eurochemic Reprocessing Plant

- Startup of Eurochemic Plant

INTERNATIONAL RELATIONSHIPS

- Member of European Communities, IAEA, and OECD-NEA.

- Partnership in Belgian-Dutch-German FBR project.

- Partnership in Eurodif and Coredif enrichment projects.

- Cooperative waste management agreement with U.S. DOE.

- Home of the Eurochemic Company and facilities (transferred to Belgian ownership in 1980). 
ORGANIZATION

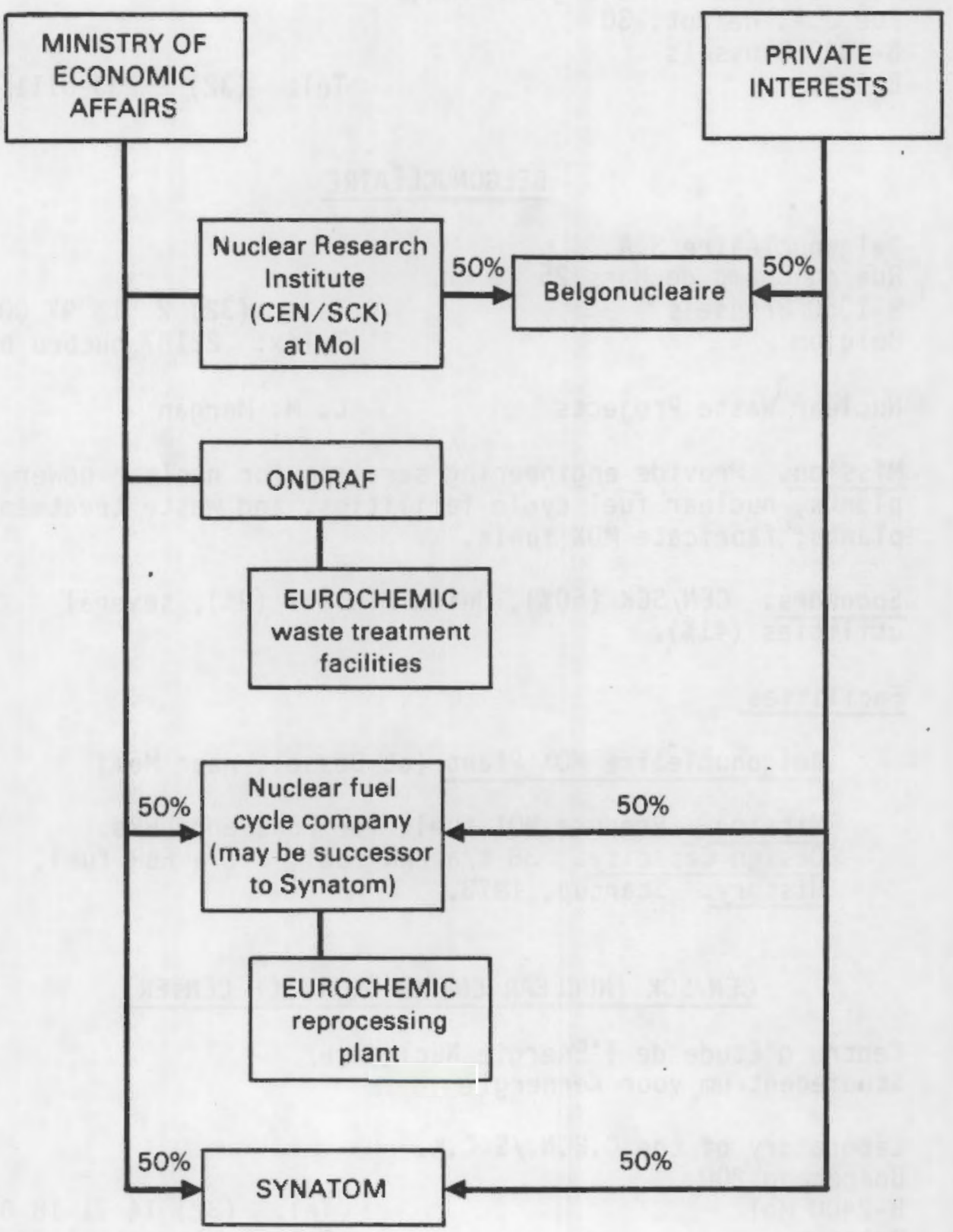


$\underline{\text { Belgium }}$

\section{BELGIAN ATOMIC ENERGY COMMISSION}

Commissariat à l'Énergie Atomique

Rue J.A. de Mot, 30

B-1040 Brussels

Belgium

Te1: (32) 2 233-6111

\section{BELGONUCLÉAIRE}

Belgonucléaire S.A.

Rue du Champ de Mars 25

B-1050 Brussels

Belgium

Tel: (32) 25139700

Telex: 22187 nucbru b.

Nuclear Waste Projects

L. M. Mergan

Mission. Provide engineering services for nuclear power plants, nuclear fuel cycle facilities, and waste treatment plants; fabricate MoX fuels.

Sponsors. CEN/SCK (50\%), Union Minière (9\%), several utilities (41\%).

\section{Facilities}

Belgonucléaire MoX Plant (at Dessel, near Mol)

Mission. Produce MOX fuels for FBRs and LWRs. Design Capacity. $38 \mathrm{t} / \mathrm{a}$ LWR fuel, 7 t/a FBR fuel. History. Startup, 1973.

\section{CEN/SCK (NUCLEAR ENERGY RESEARCH CENTER)}

Centre d'Etude de T'Energie Nucleaire/

Studiecentrum voor Kernergie

Laboratory of the C.E.N./S.C.K.

Boeretang 200

B-2400 Mol

Tel: (32) $1431 \quad 1801$

Belgium

Telex: Atomol 31922

General Manager

Deputy General Manager

Chemistry Department

Waste Programs

Geological Disposal Projects

Prof. A. S. Amelinckx

Prof. P. Dejonghe

Dr. L. H. Baetslé

N. L. C. Van de Voorde

$R$. Heremans 


\section{CEN/SCK (NUCLEAR ENERGY RESEARCH CENTER) (contd)}

Sponsor. Federal government--Ministry of Economic Affairs and Industry.

Fuel Cycle R\&D. FBR fuel reprocessing (head-end and of $f-$ gas treatment), incineration of TRU wastes, immobilization of cladding hulls, LLW treatment, geologic waste isolation in clay formations.

\section{Facilities}

1. HERMES Pilot Plant (Head-End Research facility on Mockup Engineering scale)

Mission. Develop head-end and of fgas treatment technology for LWR fuels. Design Basis. Chop-leach or chop-grind-leach; silver zeolite and cryogenic treatment of offgas. Process Components. Single-pin chopper, critically safe dissolver, mechanical pulverizer, centrifugal filter for solution clarification, fuel residue dissolver, "super dissolver" for cleanup of hulls, offgas scrubbers, silver zeolite bed, cryogenic unit. Throughput. $10 \mathrm{~kg}$ irradiated fuel $\left(20-30 \% \mathrm{PuO}_{2}\right.$ in $\mathrm{UO}_{2}$ ) per batch. History. Hot operation, early 1982.

2. FLK Slagging Incinerator (radioactive)

Mission. Volume reduction of combustible lowactivity TRU wastes.

Design Basis. High-temperature combustion (1200$1500^{\circ} \mathrm{C}$ ); capacity, $50-100 \mathrm{~kg} / \mathrm{hr}$; product, granular slag.

History. First tests with Pu-bearing wastes (tens of grams Pu in several tons of waste), 1983.

3. CEN/SCK Bituminization Plant

Mission. Immobilize Belgian LLW. Design Basis. Stirred evaporator, batch process; capacity, $60-80 \mathrm{l} / \mathrm{hr}$ liquid LLW or $15-20 \mathrm{l} / \mathrm{hr}$ dried sludge. History. Startup, 1964. 
Belgium

CEN/SCK (NUCLEAR ENERGY RESEARCH CENTER) (contd)

4. Underground Research Laboratory

Mission. Develop technology for disposal of ILW, TRU waste, and HLW in clay repositories. Description. Access shaft to $-215 \mathrm{~m}$ level, $4.5 \mathrm{~m}$ dia; laboratory gallery, $3.5 \mathrm{~m}$ useful dia by $30 \mathrm{~m}$ length; cast iron liner.

Test Program. Water-flow measurements, in situ heater tests, clay stability studies, liner stresses, borehole atmospheres, corrosion; test emplacement of HLW and TRU incinerator residues.

Milestone. Laboratory operational, early 1984.

\section{EUROCHEMIC}

Eurochemic B-2400 Mol

Tel: (32) $14312 \quad 861-70$

Belgium

Telex: EUROCHEM ML 31924

[Brussels National Airport (Zaventem); then by rental car, Eurochemic driver (1-1/2 hours), or train to Mol.]

$\begin{array}{cl}\text { Managing Director } & \text { Dr. E. J. Detilleux } \\ \text { Deputy Director } & \text { H. Eschrich } \\ \text { Industrial Development } & \text { J. Van Geel } \\ \text { Laboratories } & \\ \begin{array}{c}\text { Plant Operation } \\ \text { Department }\end{array} & \text { Werner Hild }\end{array}$

Mission. Treatment of reprocessing plant wastes. Facilities and site transferred to Belgian ownership in 1980.

Technical Activities. Ceramic melter development (technical support to FRG PAMELA pilot plant being built at Mol), glass marble encapsulation in lead matrices, and non-HL waste conditioning.

Facilities

1. Eurowetcomb (hot pilot plant-acid digestion)

Mission. Wet combustion of combustible TRU wastes. Design Basis. Acid digestion with $\mathrm{H}_{2} \mathrm{SO}_{4}-\mathrm{HNO}_{3}$. History. Startup, 1980. 


\section{EUROCHEMIC (contd)}

2. Eurobitum (bituminization plant)

Mission. Immobilize ILW.

Design Basis. Batch chemical pretreatment; screw

extruder-evaporator (continuous); capacity, $650 \mathrm{~m}^{3} / \mathrm{a}$ ILW.

History. Startup, 1978; on-stream time, $80 \%$ through July 1980.

3. Eurowatt (hot pilot plant-solvent treatment)

Mission. Treat Purex (TBP-kerosene) solvent.

Design Basis. Extract TBP with concentrated $\mathrm{H}_{3} \mathrm{PO}_{4}$, pyrolyze $\mathrm{H}_{3} \mathrm{PO}_{4}$ fraction; capacity, $1 \mathrm{~m}^{3} /$ day. History. Startup, early 1980.

4. Eurochemic Fuel Reprocessing Plant

Original Mission. Reprocess low- and high-enriched metal and oxide fuels from test reactors for Eurochemic partners.

Original Design Basis. Chemical decladding; PUREX

flowsheet; contact maintenance. Capacity, $350 \mathrm{kgJ} /$

day for magnesium- or aluminum-clad fuels, $20 \mathrm{kgU} / \mathrm{day}$

for SS- or $\mathrm{Zr}-\mathrm{clad}$ fuels, $5-10 \mathrm{kgJ}-\mathrm{Al} /$ day for HEU

fuels.

Operating History. Operated from 1966-1974 and

treated: 180 tU in low-enriched fuels, including $100 \mathrm{tU}$ in LWR/oxides, to yield $67 \mathrm{~m}^{3} \mathrm{HLW} ; 30 \mathrm{t} \mathrm{high-}$ enriched fuels, to yield $806 \mathrm{~m}^{3} \mathrm{HLW}$. The plant has been decontaminated and is now under Belgium ownership. Renovation and recommissioning, with a target startup date of $1988 / 9$ is currently under study by a Belgian (SYNATOM)-French (Cogema)-German (DWK) consortium.

Renovation Design Basis. $600 \mathrm{kgU} /$ day; chop-leach head-end for LWR fuels.

5. ALONA Acid Digestion Plant (see FRG/KfK).

6. PAMELA HLW Vitrification Pilot Plant (see FRG/DWK-KEWA). 
Service Géologique de Belgique

Jennerstraat 13

1040 Brussels

Belgium

Te1: (32) 26492094

Director-Geologist

J. Bouckaert

Nuclear Waste

N. Vandenberghe

H. Goethals

M. Van den Broeck

MINISTRY OF PUBLIC AND FAMILY HEALTH

Ministère de la Santé Publique

Service de Protection des Radiations Ionisantes

Quatier Vésale

B-1010 Brussels Tel: (32) 2 564-1755

Belgium

Telex: 21034 IHEBRU

\section{ONDRAF (NATIONAL INSTITUTE FOR RADIOACTIVE WASTES AND FISSILE MATERIALS)}

Organisme National des Déchets Radioactifs

et des Matières Fissiles (ONDRAF)

Boulevard du Regent 54

B-1000 Brussels

Tel: (32) 25137460

Be1 gium

Telex: 65784 Nirond

Chairman, Board of Directors

M. Frerotte

Chairman, Permanent Technical Committee

F. Deconinck

Director

Technical Manager \& Deputy to General

E. Detilleux

Manager in charge of Exploitation

$\mathrm{F}$. Decamps

G. Claes

Sponsor. Federal government.

Mission. Provide for transportation of radioactive mate-

rials; waste conditioning and interim storage; spent fuel

AFR storage; waste disposal; Pu storage; definition of waste management $R \& D$ requirements. Operate Eurochemic waste treatment facilities.

Organization. ONDRAF (NIRAS in Dutch) is governed by a Board of Directors composed of a president, vicepresident, and board members representing various national ministries and local government executives. The Board will be advised by a permanent Technical Committee. 
ONDRAF (NATIONAL INSTITUTE FOR RADIOACTIVE WASTES AND FISSILE MATERIALS) (contd)

\section{Facilities}

AVB (vitrification plant)

Mission. Solidify stored Eurochemic HEWC and future Belgian HLW. Design Basis. French AVM process: rotary calciner and metallic melter; capacity, $30 \mathrm{l} / \mathrm{hr}$ feed; product, borosilicate $\mathrm{glass}$ blocks, $0.4 \mathrm{~m}$ dia $\times 1.5 \mathrm{~m}$ high. Milestone. Startup, 1987 (will not be built if Eurochemic reprocessing plant is not recommissioned).

\section{SYNATOM}

\section{SYNATOM S.A.}

Avenue Marnix, 13

B-1050, Brussels

Belgium

General Manager

Fuel Reprocessing Service

Dr. Pierre A. Erkes

Jean Danguy

Mission. Provide commercial fuel cycle and waste management services for the Belgian nuclear utilities.

Sponsors. Government (50\%), INTERCOM (20\%), EBES (20\%), UNERG (10\%).

\section{US EMBASSY - BRUSSELS}

USEC Brussels

American Embassy

40 , Boulevard du Regent, $B 3$

B-1000 Brussels

Belgium

Tel: (32) 2 513-3830

US DOE Representative;

Energy and Resources 'Office

Telex: $846-21336$

Dana M. Marshall 

BRAZIL 
BRAZILIAN HOLIDAYS

*Asterisks denote movable holidays

January 1 New Year's Day

*February

Carnival

*March/April

Good Friday

April 21

Tiradentes Day

May 1

Labor Day

*June

Corpus Christi Day

September 7

Independence Day

November 2

All Souls' Day

November 15

Proclamation of the Republic

December 25

Christmas

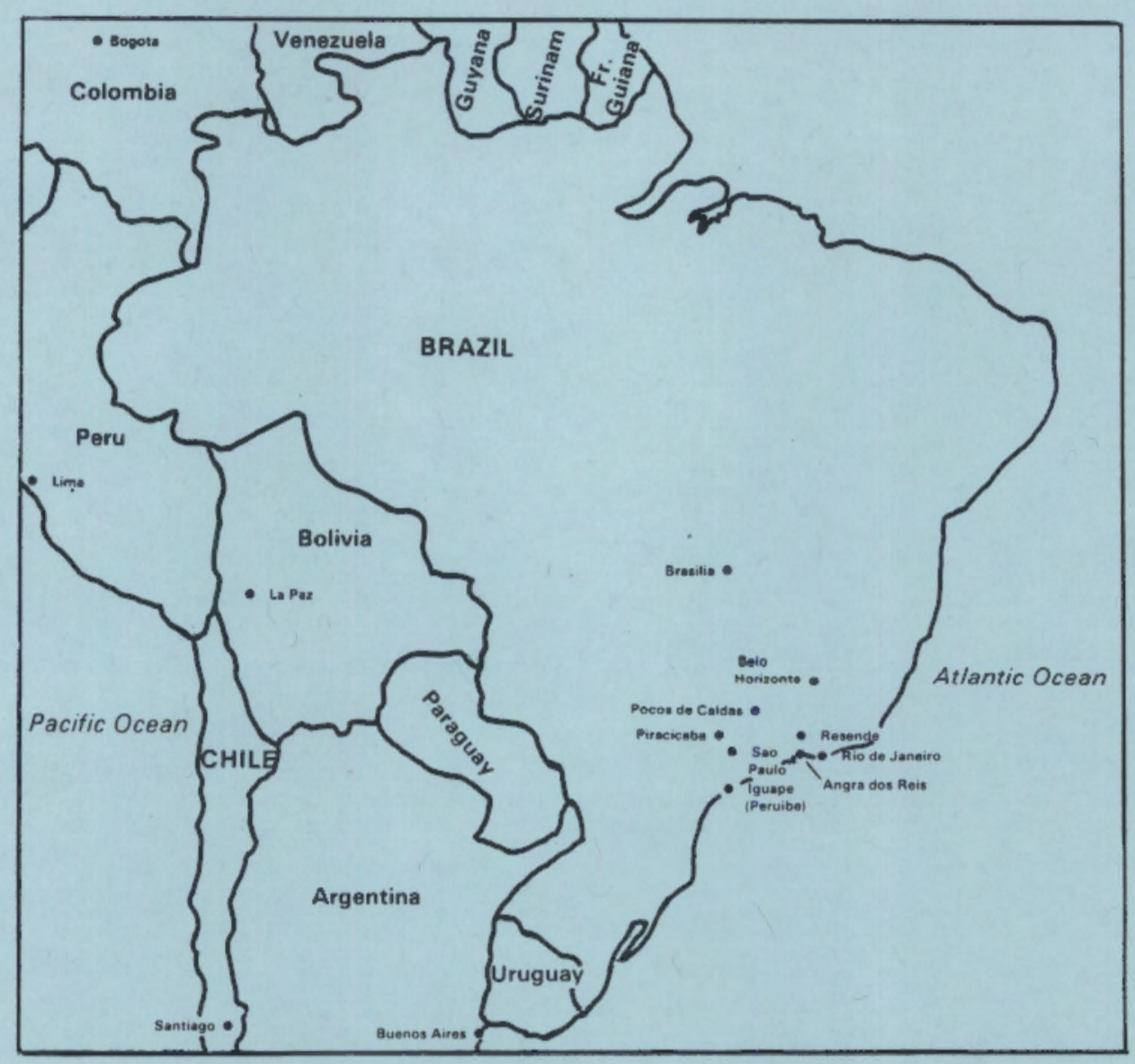




\section{BRAZIL}

ENERGY

$\begin{array}{lcc}\text { Population } & 1982 & 122 \text { million } \\ \text { Energy Demand } & 1980 & \sim 95 \text { Mtoe } \\ \text { Indigenous Energy Sources: limited crude oil, shale oil, } \\ \text { natural gas, and coal. 0il imports }-80 \% . \\ \text { Electric Power Plant Capacity } & 1982 & \sim 30 \mathrm{GWe} \\ & 1987 & 60 \mathrm{GWe} \\ & 1980 & 125 \text { TWh } \\ \text { Electric Power Production } & 1985 & 88 \% \text { hydro } \\ & & 8 \% \text { nuclear } \\ & & 4 \% \text { thermal }\end{array}$

NUCLEAR POWER

National Policy. Ambitious program to develop complete government-owned nuclear industry, based upon technology transfer from FRG and other countries.

$\begin{array}{lll}\text { Nuclear Power Plant Capacity } & 1982 & 0.6 \mathrm{GWe} \\ 1988 & 1.9 \mathrm{GWe} \\ 2000 & 4.4 \mathrm{GWe}\end{array}$

INDUSTRIAL FUEL CYCLE

Policy. Full commercial capability through a governmentowned corporation--conversion of $\mathrm{U}_{3} \mathrm{O}_{8}$ to $\mathrm{UF}_{6}$; enrichment; $\mathrm{UO}_{2}$ fuel fabrication; fuel reprocessing.

Waste Management Strategy. Not yet defined. 


$\begin{array}{clr}\text { Cumulative Spent Fuel } & 1985 & 32 \mathrm{tU} \\ \text { Arisings (LWR) } & 1990 & 180 \mathrm{tU} \\ & 1995 & 510 \mathrm{tU} \\ & 2000 & 1,000 \mathrm{tU}\end{array}$

Demonstration/Production Activities

1. Uranium mining and milling: $500 \mathrm{tU} / \mathrm{a}--i$ in operation.

2. UF 6 production: $2000 \mathrm{tUF}_{6} / \mathrm{a}$ (1984).

3. Uranium enrichment (Becker nozzle process):

First Cascade, 24 stages (1984)

200-300 tSWU/a demo plant (1988).

4. Fuel fabrication: $80 \mathrm{t} / \mathrm{a}$ (1982); design capacity-$400 \mathrm{tU} / \mathrm{a}$.

5. Spent fuel reprocessing: $10 \mathrm{~kg} / \mathrm{d}$ pilot plant (1986).

INTERNATIONAL RELATIONSHIPS

1. A member state of IAEA--but has not signed NPT.

2. Depends heavily upon nuclear technology transfer (mainly from FRG).

3. Joint US-Brazil R\&D program to study radionuclide transport from a thorium deposit.

4. Current interest in receiving US waste management technology.

ORGANIZATION

Federal Ministry of Mines and Energy: planning, execution and control of nuclear power program.

- Nuclear Energy Commission (CNEN): regulatory and R\&D

- Nuclebrás: plant engineering, construction and operation; Nuclebrás operates through partly-owned subsidiaries--Nucon (construction), Nuclen (design), Nuclep (heavy components), Nuclam (mining), Nuclei and Nustep (enrichment), and Nuclemen (thorium production). 


\section{CENTER FOR THE DEVELOPMENT OF NUCLEAR TECHNOLOGY}

Centro de Desenvolvimento de

Tecnologia Nuclear de Nuclebrás (CDTN)

Rua Goncalves Dias No. 1054

Belo Horizonte, MG

Brazil

Te1: (55) 31 226-6988

Mission. Applied research and industrial development of uses of atomic energy.

\section{CNEN (NUCLEAR ENERGY COMMISSION)}

CNEN (Comissao Nacional de Energía Nuclear)

Rua General Severiano 90

Botafogo ZC-82

20000 Rio de Janeiro, RJ

Brazil

Tel: (55) 21 246-1747

Telex: 2121280

President

Hervásio Guimaraes de Carvalho

Fuel Cycle and Waste Management R\&O. Uranium enrichment; migration of radionuclides from a thorium deposit, a joint Brazil-US study; spent fuel reprocessing. The government is building a $10 \mathrm{~kg} \mathrm{HM} /$ day pilot reprocessing facility in the Rio de Janeiro area.

\section{IPEN}

Instituto de Pesquisas Energéticas e Nucleares

(Energy and Nuclear Research Institute)

Cidade Universitária

Caixa Postal 11.049

Pinheiros - CEP 01000

Sao Paulo, Brazil

Te1: (55) 112116011

Superintendent

Telex: (011) 23592-IPEN

Mission. Nuclear R\&D. 


\section{IPEN (contd)}

Areas of Activity. Physics; nuclear physics; high energy physics. Life Sciences; nuclear medicine; radiobiology; radiation health and safety. Engineering and reactor technology; instrumentation. Chemistry; materials in the nuclear industry. Isotope and radiation applications and production. Nuclear waste disposal. Nuclear metallurgy. Radiochemistry.

\section{NUCLEBRÁS}

Nuclebrás

Avenida Presidente Wilson 231

CEP 20030, Rio de Janeiro

Tel: (55) $21292-1144$

Brazil

Telex: 23128,23830

President

Dario Gomes

Director

John Forman

Mission. Establish a nuclear fuel cycle industry, promote nuclear technology, transfer to private industry, design and build nuclear power plants, pursue R\&D programs.

Sponsor. Federal government. 
CANADA 


\begin{tabular}{|c|c|}
\hline \multicolumn{2}{|c|}{ CANADIAN HOLIDAYS } \\
\hline *Asterisks & movable holidays \\
\hline January 1 & New Year's Day \\
\hline *March/April & Good Friday \\
\hline \#March/April & Easter Monday \\
\hline *May & $\begin{array}{c}\text { Victoria Day (Monday } \\
\text { preceding May 25) }\end{array}$ \\
\hline July 1 & Canada Day \\
\hline *September & $\begin{array}{l}\text { Labor Day (1st Monday } \\
\text { in September) }\end{array}$ \\
\hline *October & $\begin{array}{l}\text { Thanksgiving Day } \\
\text { (2nd Monday in October) }\end{array}$ \\
\hline December 25 & Christmas \\
\hline December 26 & $\begin{array}{c}\text { Boxing Day (observed in all } \\
\text { provinces except Quebec) }\end{array}$ \\
\hline & TE \\
\hline November 11 & $\begin{array}{l}\text { Remembrance Day observed in } \\
\text { all provinces but most } \\
\text { businesses remain open }\end{array}$ \\
\hline
\end{tabular}

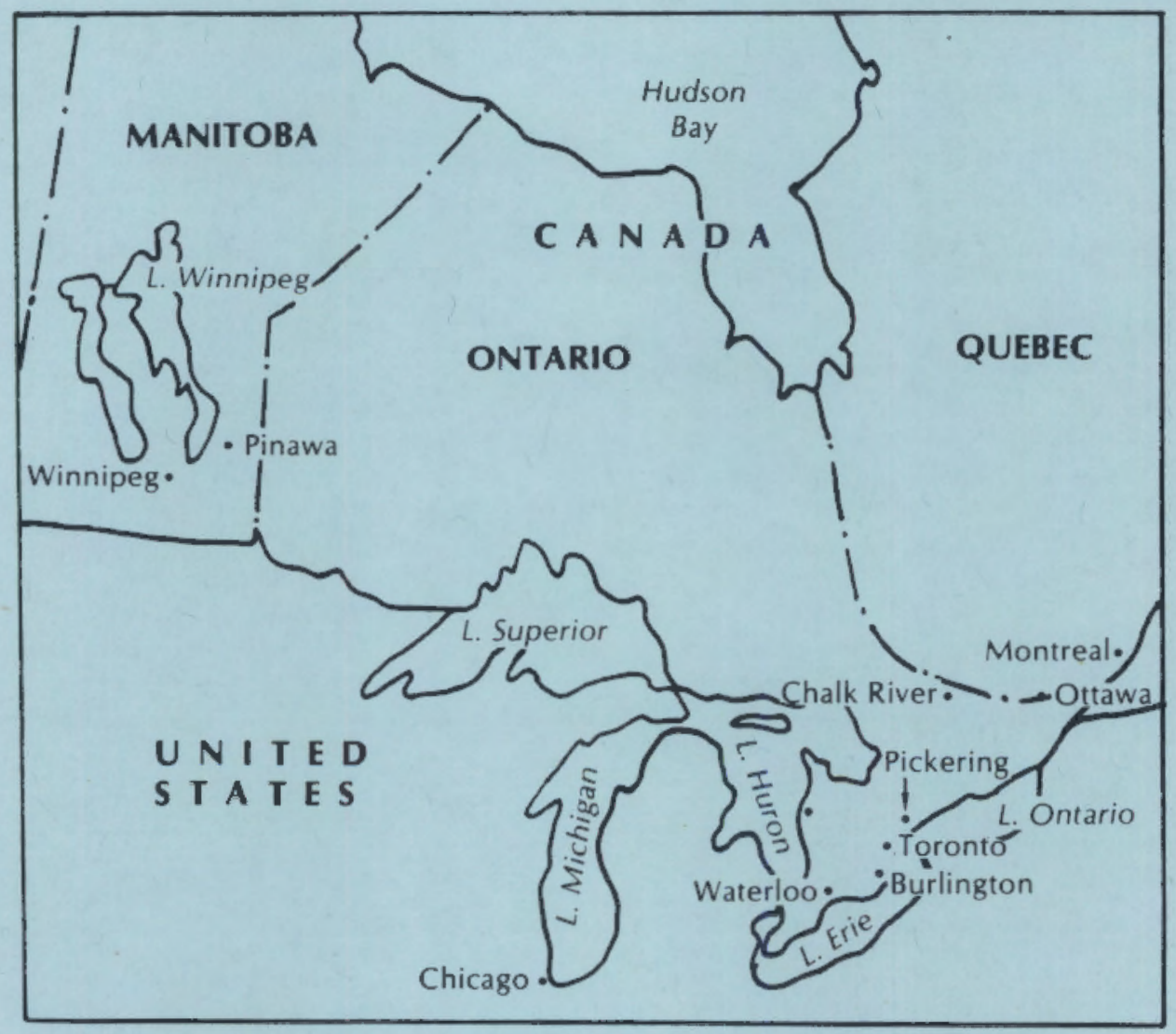


ENERGY

$\begin{array}{lcl}\begin{array}{l}\text { Population } \\ \text { Energy Demand }\end{array} & 1982 & 24.3 \text { million } \\ \text { Oil Demand } & 1979 & 281.3 \text { Mtoe } \\ & 1979 & \begin{array}{l}90.4 \text { Mtoe } \\ (9 \% \text { imported })\end{array} \\ \text { Electric Power Plant Capacity } & 1980 & 79 \text { GWe } \\ \text { Electric Power Production } & 1982 & \begin{array}{l}375 \text { TWh-- } \\ 10 \% \text { nuclear }\end{array} \\ & & 68 \% \text { hydro } \\ & & 22 \% \text { thermal }\end{array}$

NUCLEAR POWER

National Policy. Strong support of the domestic use and the export of the CANDU reactor system, with possible conversion to a thorium/uranium-233 fuel cycle.

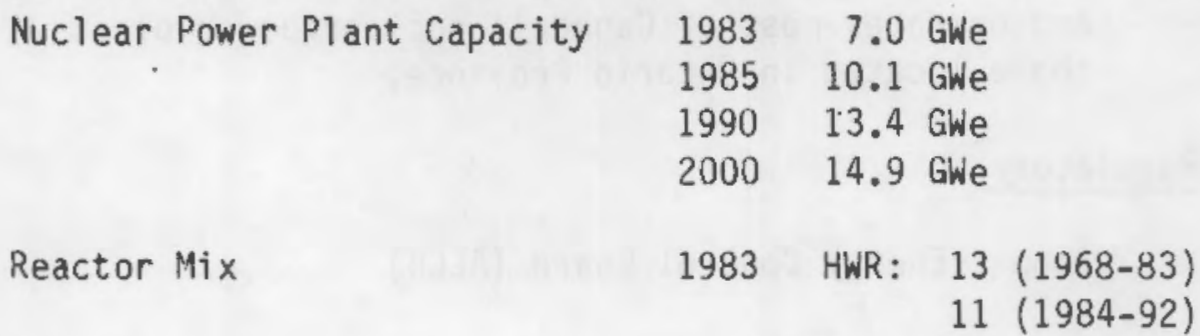

INDUSTRIAL FUEL CYCLE

Policy. Retrievable storage of spent fuels, pending decision as to reprocessing for recovery and recycle of $\mathrm{Pu}$ or reprocessing to implement the Th/U fuel cycle; continue small reprocessing $R \& D$ activity.

Waste Management Strategy. Geologic disposal of "nuclear fuel waste," either spent CANDU fuel or immobilized HLW, in a crystalline rock repository; disposal of reactor wastes in engineered surface facilities. 


$\begin{array}{crr}\text { Cumulative Spent Fuel } & 1980 & 3,650 \mathrm{tU} \\ \text { Arisings (HWR) } & 1985 & 8,800 \mathrm{tU} \\ & 1990 & 17,700 \mathrm{tU} \\ & 2000 & 38,000 \mathrm{tU}\end{array}$

Major Milestones

- Start of shaft excavation, Underground Research Laboratory (URL)

- Spent fuel immobilization pilot plant

- Complete concept verification for a 1990 geologic repository

ORGANIZATION

Commercial Nuclear Enterprise

1. Atomic Energy of Canada Limited (AECL), a Crown Corporation owned by the federal government. Nuclear R\&D; design, engineering and sale of CANDU reactors; operation of heavy-water plants.

2. Ontario Hydro, provincial public utility which owns and operates most of Canada's nuclear power plants-those located in Ontario Province.

Regulatory

Atomic Energy Control Board (AECB)

WASTE MANAGEMENT R\&D

- AECL-Whiteshell and Chalk River

- Ontario Hydro

- Supporting studies by various other governmental agencies, consulting firms and universities. 


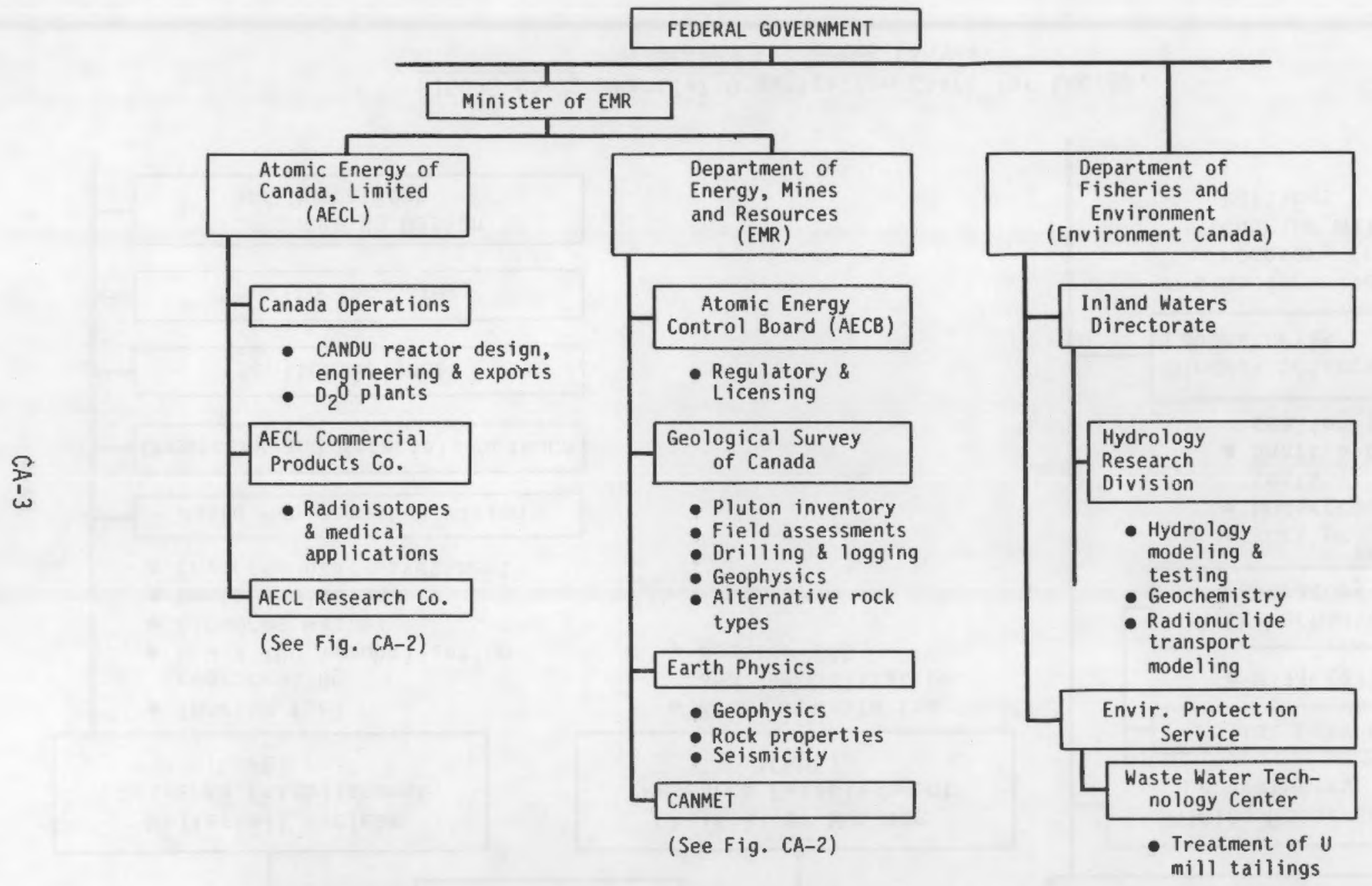

FIGURE CA-1. Government Responsibilities--Fuel Cycle and Waste Management 

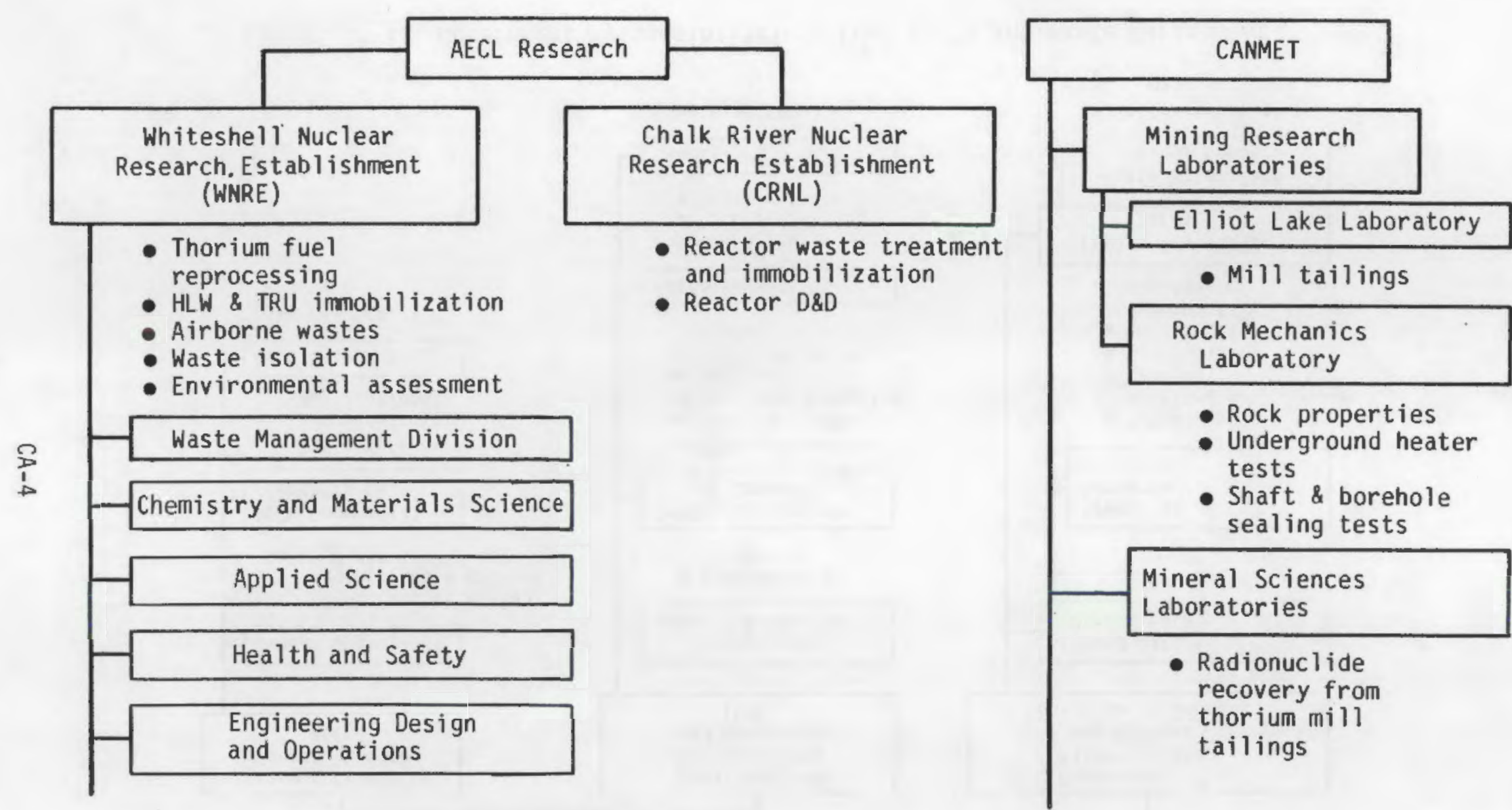

Chalk River Nuclear Research Establishment (CRNL)

- Reactor waste treatment and immobilization

- Reactor D\&D

FIGURE CA-2. Partial Organization Chart for Energy,

- Rock properties

- Underground heater tests

- Shaft \& borehole sealing tests

Mineral Sciences

Laboratories

- Radionuclide recovery from thorium mill

tailings Mines and Resources Canada 


\section{AECB}

Atomic Energy Control Board

P.0. Box 1046

270 Albert Street

Ottawa, Ontario K1P 559

Tel: (613) 995-5894

Canada

Telex: $\quad 533771$

President
Director, Fuel Cycle/Materials

J. H. Jennekens

Regulation

Waste Management Division

W. D. Smythe

L. C. Henry

(613) 995-3181

Fuel and Heavy Water Plant

J. P. Didyk

Division

Director, Regulatory Research

Branch

Safety and Safeguards Section

J. W. Bears

J. R. Coady

(613) $995-7651$

AECL

Atomic Energy of Canada Limited

275 Slater Street

Dttawa, Ontario K1A DS4

Tel: (613) $237-3270$

Canada

Telex: 0533126

Chairman

President

President, AECL Research

Vice President, R\&D

Robert Despres

James Donnelly

Robert Hart

Dr. A. J. Mooradian

\section{CANMET (CANADA CENTRE FOR MINERAL}

AND ENERGY TECHNOLOGY)

CANMET

555 Booth Street

Ottawa, Ontario K1A OGI

Canada

Tel: (613) 995-4029 
CANMET (contd)

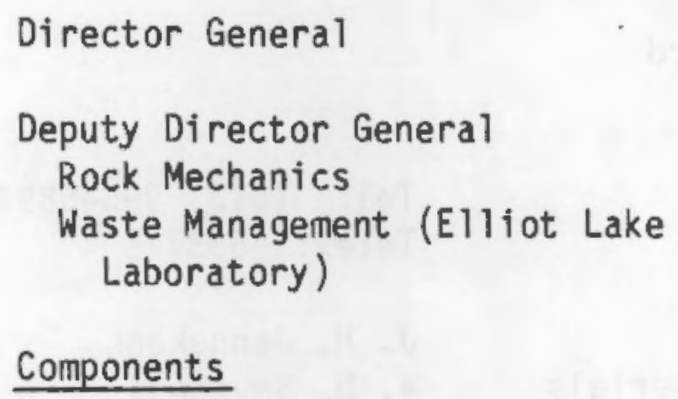

- Mineral Sciences Laboratories (Ottawa)

- Rock Mechanics Laboratory (Ottawa)

- Elliot Lake Laboratory Mining Research Laboratories

Elliot Lake, Ontario P5A 2K2 Canada

\section{CHALK RIVER}

AECL Chalk River Nuclear Laboratories

Chalk River, Ontario KOJ 1JO Canada

Te1: (613) 687-5581

Telex: 053-34555

Chalk River Environmental

Dr. D. H. Charlesworth

Authority

Director, Applied R\&D

(613) 687-2441

Dr. H. K. Rae

Systems Materials

(613) $687-2624$

Dr. G. F. Taylor

(613) $687-2771$

Facilities

Waste Treatment Centre (WTC)

Mission. Develop reactor waste treatment processes. 
EMR (ENERGY, MINES AND RESOURCES CANADA)

Energy, Mines and Resources Canada

Energy Sector

580 Booth Street

Ottawa, Ontario K1A OE4

Tel: (613) 995-3065

Canada

Telex: 0533117

Adviser, Nuclear Waste Management Joseph Howieson

Energy, Mines and Resources Canada

Earth Sciences

Geological Survey of Canada

601 Booth Street

Ottawa, Ontario K1A OE8

Canada

Tel: (613) 995-4938

Telex: 053-3117

Director

Director, Terrain Sciences

Dr. D. G. McLaren

Dr. J. S. Scott

\section{ONTARIO HYDRO}

Ontario Hydro

700 University Avenue

Toronto, Ontario M5G $1 \times 6$

Canada

Tel: (416) 592-5111

Telex: 06217662

Nuclear Design and Development Division

Nuclear Materials Management

Department

Reactor Waste Management

D. A. Meneley

(416) $592-5210$

H. N. Isaac

(416) 592-5539

T. J. Carter

Irradiated Fuel Management

(416) 592-6024

R. C. Oberth

(416) 592-2856

Mission. Design, construction, and operation of CANDU power stations. 


\section{ONTARIO HYDRO (contd)}

\section{Facilities}

1. RWVRF (Radioactive Waste Volume Reduction Facility)

Location. Bruce Nuclear Complex, Tiverton, Ontario. Components. Pyrolysis incinerator (2-chamber), solid waste compactor. History. Startup, 1977.

2. Intermediate-Level Waste Storage

Location. Bruce Nuclear Power Development Waste Management Site, Tiverton, Ontario. Components. In-ground concrete tile holes or trenches, above-ground concrete "Quadricel1."

\section{WASTEWATER TECHNOLOGY CENTRE}

Wastewater Technology Centre

Environmental Protection Service

Department of Fisheries and the Environment

P.0. Box 5050

Burlington, Ontario L7R 4A6

Canada

\section{WHITESHELL}

Atomic Energy of Canada Limited (AECL)

Whiteshell Nuclear Research

Establishment

Pinawa, Manitoba ROE 1 LO

Tel: (204) 753-2311

Canada

Telex: 0757553 


\section{WHITESHELL (contd)}

$\begin{array}{ll}\text { Vice President and General Manager } & \text { R. E. Green } \\ \text { Director, Chemistry and Materials } & \text { M. Tomlinson } \\ \text { Science } & \text { W. T. Hancox } \\ \text { Director, Applied Science } & \text { D. R. McLean } \\ \text { Fuel Recycle } & \text { S. A. Mayman } \\ \text { Engineering Design } & \\ \text { and Operations } & \text { T. E. Rummery } \\ \text { Director, Waste Management } & \text { (2D4) 753-8432 } \\ \begin{array}{ll}\text { Scientific Assistant to the } & \text { E. L. J. Rosinger } \\ \text { Director } & \text { K. Nuttall } \\ \text { Director, Fuel Waste } & \text { F. P. Sargent } \\ \text { Technology } & \text { K. W. Dormuth } \\ \text { Director, Geochemistry and } & \text { R. B. Lyon } \\ \text { Applied Chemistry } & \\ \text { Director, Applied Geoscience } & \end{array} \\ \begin{array}{ll}\text { Director, Environmental and } \\ \text { Safety Assessment }\end{array}\end{array}$

\section{Facilities}

1. WIPE (cold pilot plant-vitrification)

Mission. Develop HLW conditioning process for the CANDU-Thorium fuel cycle.

Design Basis. $10 \mathrm{~kg} / \mathrm{hr}$ glass--rotospray calciner/ ceramic melter.

History. Startup, 1983.

2. Hot Pilot Plant-Reprocessing

Mission. Develop CANDU-Thorium fuel cycle technology; provide HLW for waste studies. Design Basis. Thorex process, mixer-settlers; capacity, $0.3 \mathrm{~kg} /$ day. History. Hot operation, 1980. 
WHITESHELL (contd)

3. URL (Underground Research Laboratory)

Mission. Provide a research facility in a virgin granite pluton characteristic of the Canadian granite formations which may be selected for waste repository construction.

Design Basis. Location about $20 \mathrm{~km}$ from the Whiteshell reservation, on the Lac du Bonnet Batholith; horizontal tunnel with adjoining rooms located $300 \pm$ $200 \mathrm{~m}$ below the surface, with vertical shaft or ramp access. Licensed radioactive sources and selected tracers may be used in the facility, but no radioactive wastes are to be emplaced there. Milestones. Start site evaluation, Fall 1980; start URL construction, 1984; start underground operation, 1986.

Construction Cost. $\$ 19.2$ million.

4. Immobilized Fuel Test Facility (IFTF)

Mission. Experimental studies of the behavior of spent fuels and HLW forms in a radiation field. Test Beds. Concrete canisters, $2 \mathrm{~m}$ dia $\times 2 \mathrm{~m}$ high, which hold heated pressure vessels containing individual experiments. History. Licensing and commissioning were under way in May 1983. 
Canada

\section{WINNIPEG AIRPORT TO PINAWA (120 km)}

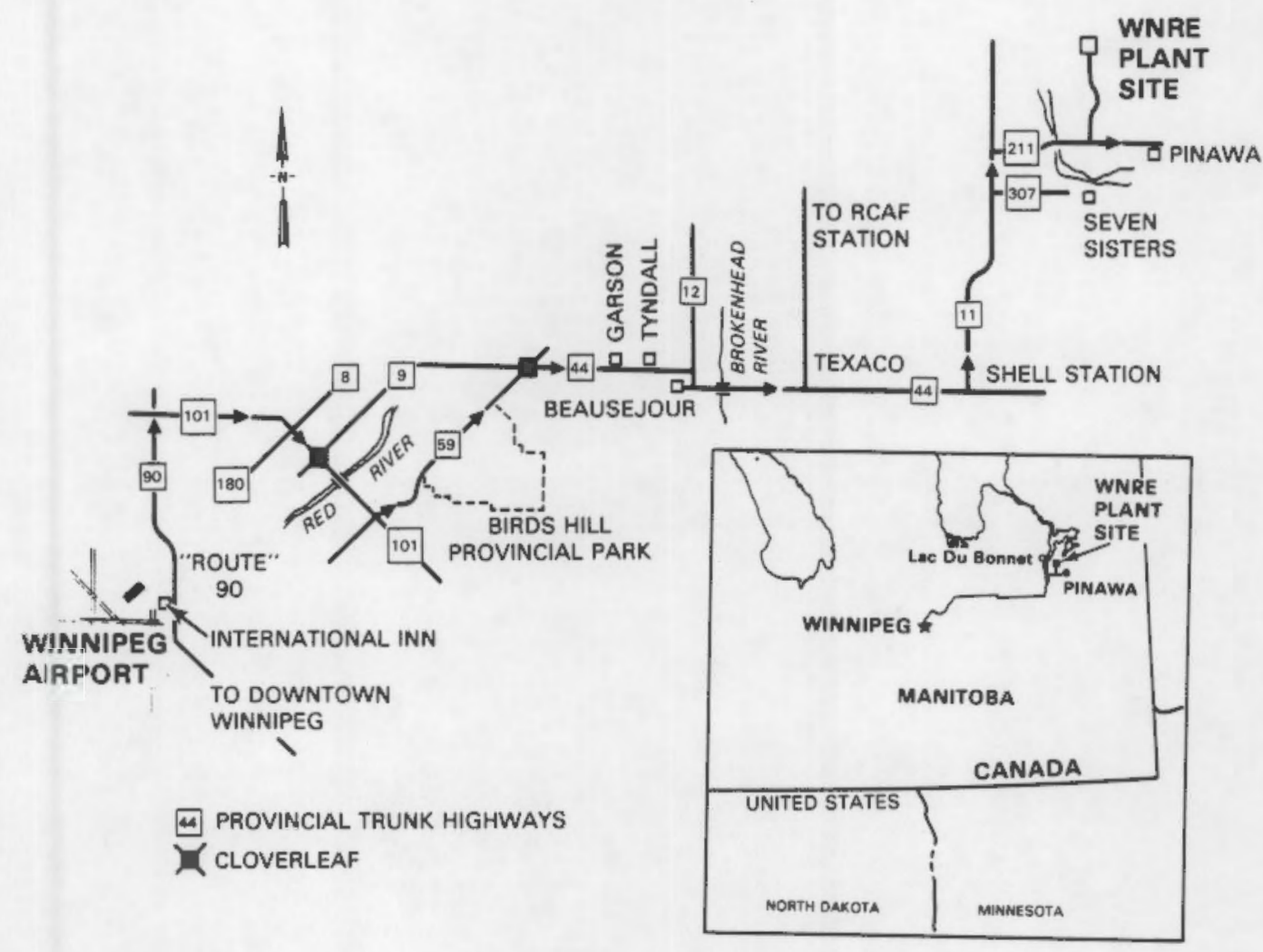





\section{CHINA}

(People's Republic of) 


\section{CHINESE HOLIDAYS}

Late January/ Lunar New Year

early February

May 1

Aug 1

Det 1-2

Labor Day

Army Day

National Liberation Days

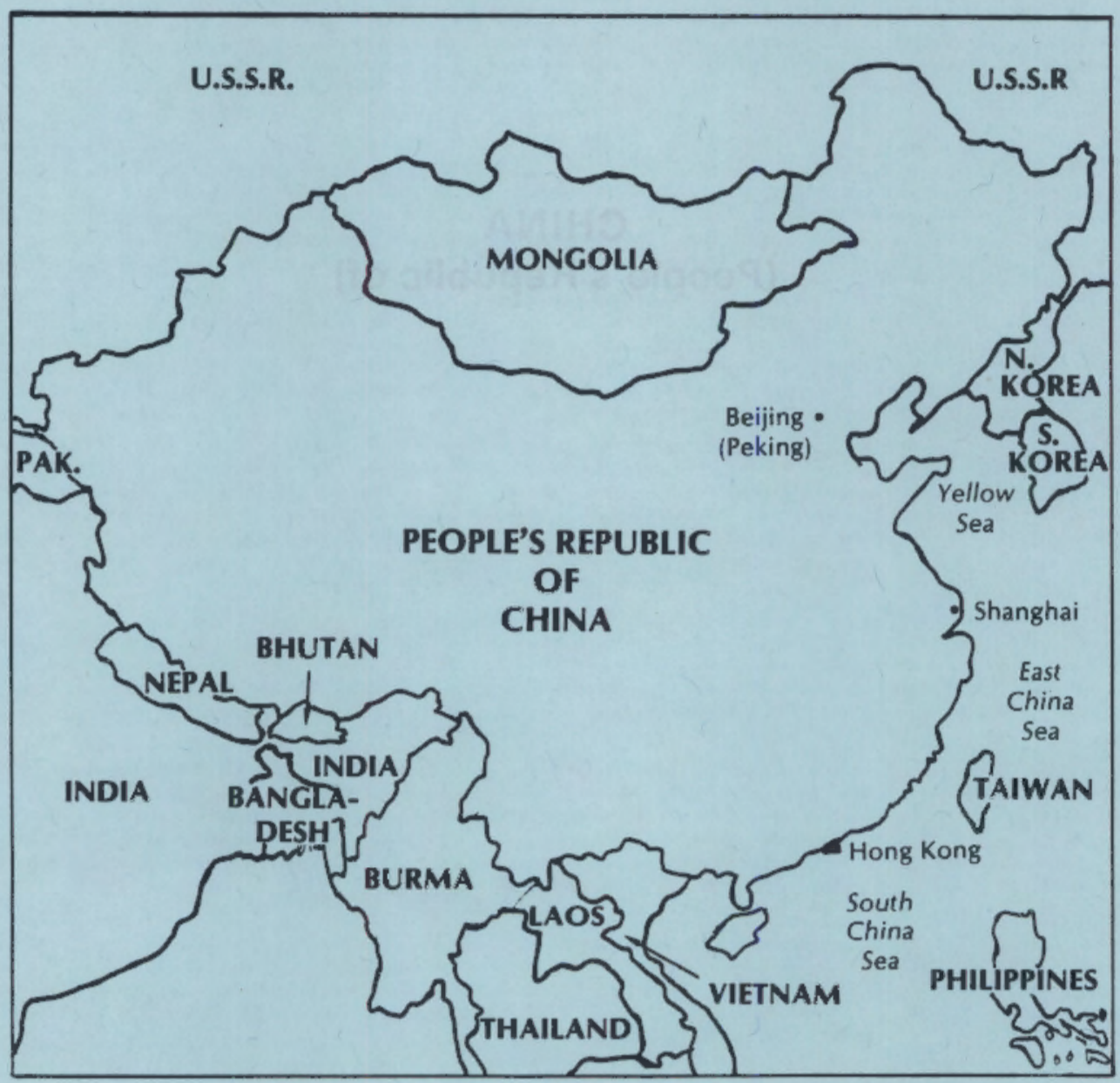


ENERGY

Population

Energy Demand

Electric Power Plant

Capacity

Electric Power Production
1982

1980

$$
\begin{gathered}
637.2 \text { Mtce-- } \\
69.4 \% \text { coal } \\
23.8 \% \text { oi } 1 \\
3.0 \% \text { gas } \\
3.8 \% \text { hydro }
\end{gathered}
$$

$1980 \quad 55-60$ GWe

$1980 \quad 300.6$ TWh

\section{NUCLEAR POWER}

National Policy. Develop nuclear power as one of three major sources of energy, in response to imperative need to solve problems caused by uneven distribution of resources; be self-sufficient, but introduce foreign advanced technology.

Nuclear Power Plant Capacity

Reactor Mix

Reactor Development
1990 2000

2 GWe 10 GWe

1983 PWR: 7 in construction or planning stage

HWR, BWR, HTGR

INDUSTRIAL-SCALE FUEL CYCLE ACTIVITIES

Uranium mining, milling and enrichment; fuel fabrication, reprocessing of defense fuels.

\section{ORGANIZATION}

- Ministry of the Nuclear Industry--all fields of nuclear power development

- Atomic Energy Research Institute of the Academy of Sciences (Peking Area)--nuclear R\&D. 


\section{INSTITUTE OF ATOMIC ENERGY}

Institute of Atomic Energy

Academia Sinica, Beijing

People's Republic of China

Director

Wang Ganchang

\section{INSTITUTE OF NUCLEAR ENERGY TECHNOLOGY}

Institute of Nuclear Energy Technology

Qinghua University

P.0. Box 1021

Beijing, People's Republic of China

Director

Deputy Director, Division, Radiochemistry Technology

Prof. Lu Yingzhong

Prof. Zhu Yong-jun

\section{MINISTRY OF THE NUCLEAR INDUSTRY \\ (formerly the Second Ministry of Machine Building)}

Ministry of the Nuclear Industry

Beijing, People's Republic of China

Minister

Schen Gie

Vice-Minister

Chairman, Science and Techn.

Committee

Jiang Sheng-Jie

Please Note: The customary form of address may be to use the surname first, followed by the given name. In case of uncertainty it is recommended to use both names. 


\section{DENMARK}




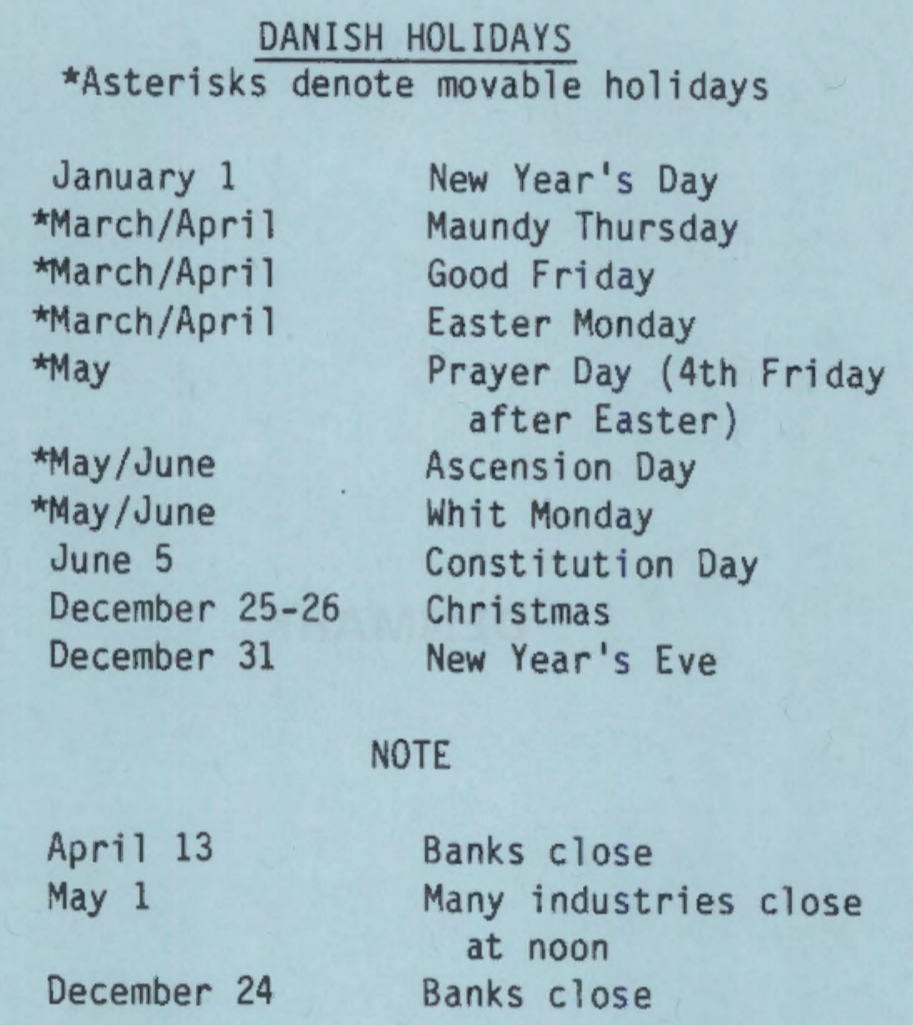

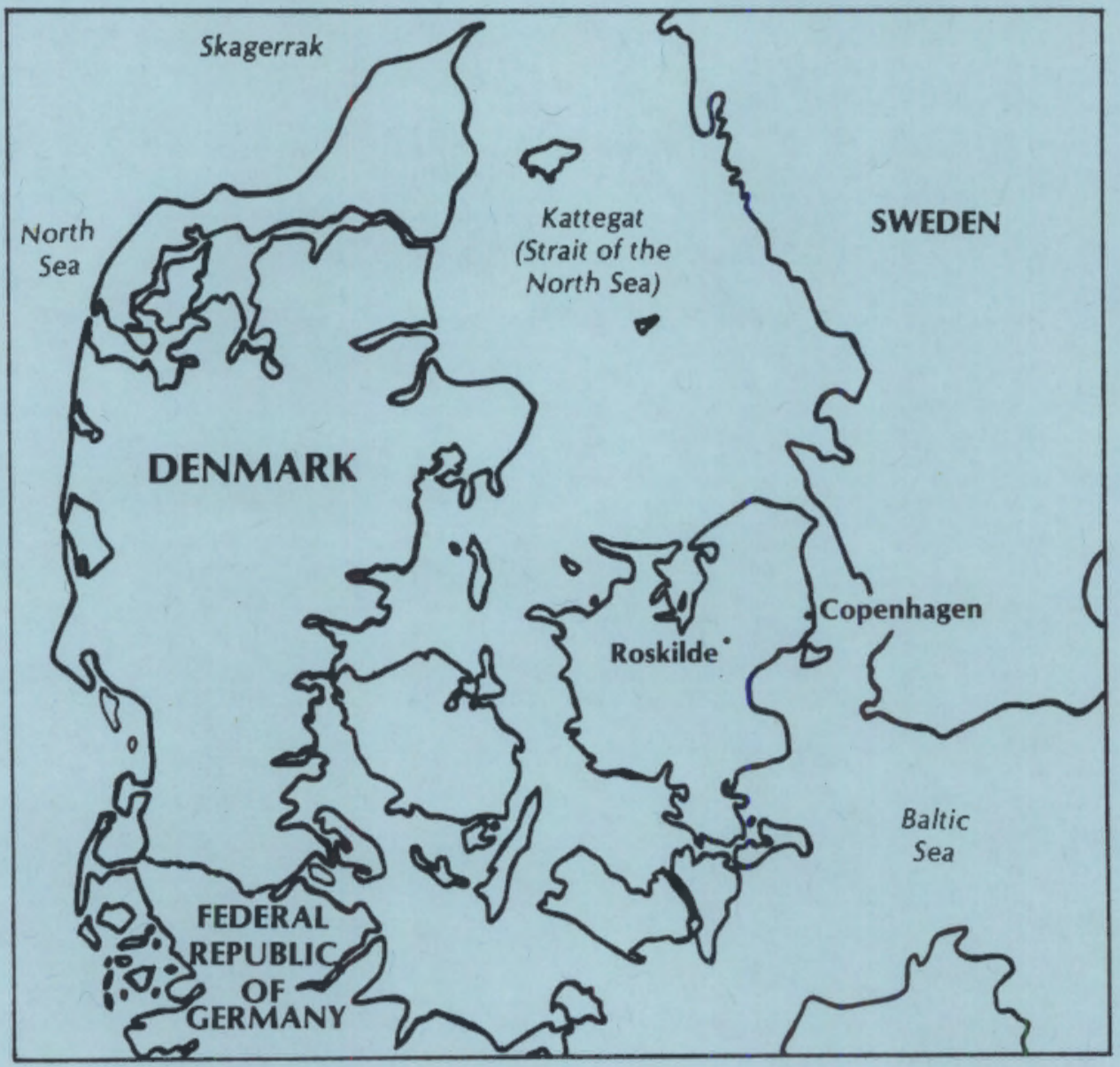




\section{DENMARK}

\section{ENERGY}

$\begin{array}{lll}\begin{array}{lll}\text { Population } & 1981 & 5.12 \text { million } \\ \text { Energy Demand } & 1980 & \begin{array}{l}19.7 \text { Mtoe } \\ (97 \% \text { imported })\end{array} \\ \text { 0il Demand } & 1980 & \begin{array}{l}13.5 \text { Mtoe } \\ (98 \% \text { imported })\end{array} \\ \text { Electric Power Plant Capacity } & 1980 & 7 \text { GWe } \\ \text { Electric Power Production } & 1980 & \begin{array}{l}27.1 \text { TWh-- } \\ 80 \% \text { solid fuels }\end{array} \\ & & 19.6 \% \text { petroleum } \\ \text { products }\end{array} \\ & & 0.1 \% \text { hydro }\end{array}$

NUCLEAR POWER

National Policy. National energy plan calls for nuclear power, but public opposition has prevented implementation; power companies continue to plan for reactor plant construction and for management of wastes.

INDUSTRIAL FUEL CYCLE

Policy. Not defined; power companies are conducting design, feasibility, and site characterization studies for a Danish salt-dome repository.

ORGANIZATION

- Danish Atomic Energy Commission--overall responsibility for nuclear energy matters

- Risф Research Establishment--nuclear R\&D

- Elkraft and Elsam (power companies)--waste disposal studies. 


\section{ATOMIC ENERGY COMMISSION}

Atomenergikommisionen

Strandgade 29

DK-1401 Copenhagen

Denmark

\section{ELKRAFT}

ELKRAFT .

Power Company Ltd., Copenhagen

Lautruph $\varnothing j 5$

P.0. Box 230

DK-2750 Ballerup

Denmark

Tel: (45) 2660022

Waste Repository Project

Finn Hasted

\section{ELSAM}

Elsam

DK-7000 Fredericia

Denmark

Tel: (45) 5562500

Telex: 51151

Waste Repository Project

Soren Mehlsen

\section{GEOLOGICAL SURVEY}

Geological Survey of Denmark

Thoravej 31

DK-2400 Copenhagen NV

Denmark 


\section{RISØ NATIONAL LABORATORY}

Ris $\varnothing$ National Laboratory

P.0. Box 49

DK-4000 Roskilde

Tel: (45) 3371212

Denmark

Telex: 43116

Chemistry Department:

Chairman

Bror Skytte Jensen

Ext. 5300

Section Leader

Knud Brodersen

Ext. 5340

Project Leader

Lars Carlsen

Ext. 5352

Mission. Scientific and technological research on atomic energy.

Sponsor. Federal government. 



\section{FINLAND}


FINNISH HOLIDAYS

*Asterisks denote movable holidays

$\begin{array}{ll}\text { January 1 } & \text { New Year's Day } \\ \text { January 6 } & \text { Epiphany } \\ \text { *March/Apri1 } & \text { Good Friday } \\ \text { *March/Apri1 } & \text { Easter Monday } \\ \text { May 1 } & \text { May Day } \\ \text { *May/June } & \text { Ascension Day } \\ \text { *May/June } & \text { Whitsunday } \\ \text { June 26 } & \text { Midsummer's Day } \\ \text { November 1 } & \text { A11 Saints'Day } \\ \text { December 6 } & \text { Independence Day } \\ \text { December 25-26 } & \text { Christmas }\end{array}$

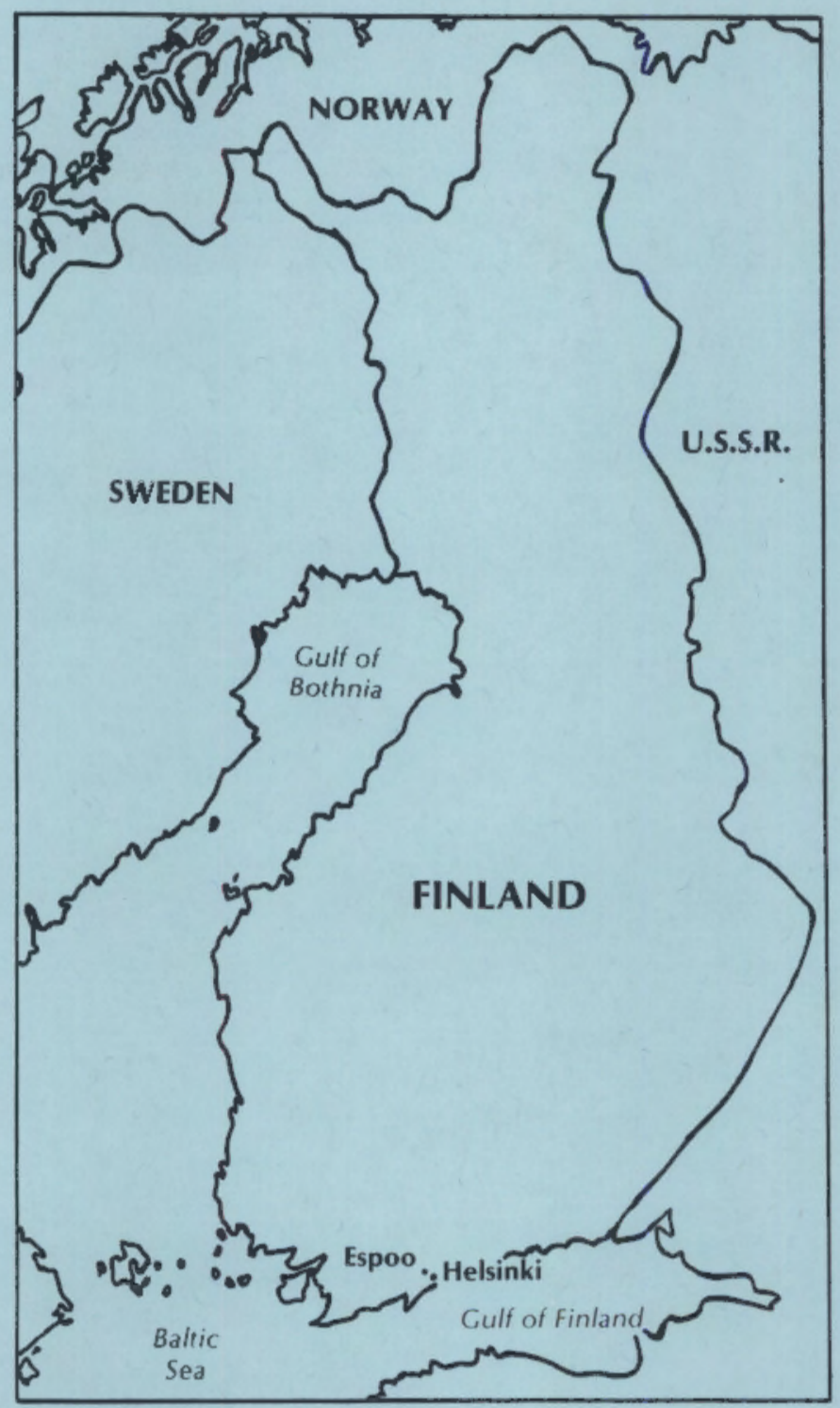




\section{F INLAND}

ENERGY

\begin{tabular}{|c|c|c|}
\hline Population & 1982 & 4.8 million \\
\hline Energy Demand & 1981 & $\begin{array}{l}25.3 \text { Mtoe } \\
\text { (73\% imported) }\end{array}$ \\
\hline $0 i 1$ Demand & 1981 & $\begin{array}{l}10.6 \text { Mtoe } \\
\text { (100\% imported) }\end{array}$ \\
\hline Indigenous Energy Sources & \multicolumn{2}{|c|}{ Hydropower, wood, peat } \\
\hline Electric Power Plant Capacity & 1982 & 11.9 GWe \\
\hline Electric Power Production & 1982 & $\begin{array}{l}39.5 \text { TWh-- } \\
40 \% \text { nuclear } \\
33 \% \text { hydro } \\
9 \% \text { coal } \\
10 \% \text { oil \& gas }\end{array}$ \\
\hline
\end{tabular}

NUCLEAR POWER

Nuclear Power Plant Capacity 19832.2 GWe

19953.2 GWe

20003.2 GWe

Reactor Mix

1982 PWR: $2(1977,1981)$

BWR: $2(1979,1982)$

INDUSTRIAL FUEL CYCLE

Policy. Purchase fuel and fuel cycle services from other countries (spent fuel from Russian-built reactors is returned to USSR).

Waste Management Strategy. Not fully defined; spent fuel or HLW from non-Russian fuels will be stored for 40 years, then placed in a granite repository; reactor wastes are bituminized and will be stored underground.

$\begin{array}{crr}\text { Cumulative Spent Fuel } & 1980 & 48 \mathrm{tU} \\ \text { Arisings (LWR) } & 1985 & 350 \mathrm{tU} \\ & 1990 & 650 \mathrm{tU} \\ & 2000 & 1,400 \mathrm{tU}\end{array}$


Major Milestones

- Geologic repository, complete site 1992 characterization

- Complete site selection 2000

- Repository completion 2020

ORGANIZATION

1. IVO and TV0: state-owned power companies; IV0 operates two Russian-built reactors, while TV0 operates two Swedish-built plants.

2. Nuclear Waste Commission of Finnish Power Companies-established by IVO and TVO to do joint analysis and planning for managing their nuclear wastes.

3. Technical Research Center (VTT)--nuclear research, including waste management R\&D.

\section{ATOMIC ENERGY COMMISSION}

Atomic Energy Commission

Ministry of Trade and Industry

Pohjois Makasiinikatu 6

SF-00130 Helsinki 13

Finl and

Telex: 125452 EDEPT SF

Chairman

Prof. Pekka Jauho

Tel: (358) 04564100

Secretary-Genera 1

Telex: 122972

Prof. Jorma Routti

Tel: (358) 04512450

Telex: 125161 


\section{GEOLOGICAL SURVEY OF FINLAND}

Geological Survey of Finland

Kivimiehentie 1

SF-02150 Espoo 15

Tel: (358) 046931

Finland

Telex: 123185 geolo SF

Director

Prof. L. K. Kauranne

Research Director

Nuclear Waste Disposal Study

Prof. K. Korpela

Mr. M. Salmi

(358) 08037988

\section{INSTITUTE OF RADIATION PROTECTION}

Institute of Radiation Protection

P.0. Box 268

SF-00101 Helsinki 10

Tel: (358) 061671

Finland

Telex: 122691 STL-SF

Department of Reactor Safety:

Chief Inspector

Inspectors

S. Våisånen

E. Ruokola

I. Aro

TECHNICAL RESEARCH CENTRE OF FINLAND

Technical Research Centre of Finland

Nuclear Engineering Laboratory

P.0. Box 169

SF-00181 Helsinki 18

Tel: (358) 04561

Finland

Telex: 125175

Director, Nuclear Waste Section Nuclear Waste Management

Dr. Esko Peltonen

Dr. Seppo Vuori

Reactor Laboratory

Otakaari 3A

SF-02150 Espoo 15

Finland

Tel: (358) 04561

Telex: 125175

Nuclear Waste Management

Dr. Pekka Hi ismaki 

FRANCE 


\begin{tabular}{|c|c|}
\hline \multicolumn{2}{|c|}{ FRENCH HOL IDAYS } \\
\hline *Asterisks & movable holidays \\
\hline January 1 & New Year's Day \\
\hline *March/April & Easter Monday \\
\hline May 1 & Labor Day \\
\hline «May/June & Ascension Day \\
\hline 太May/June & Whit Monday \\
\hline July 14 & Bastille Day \\
\hline August 15 & Assumption Day \\
\hline November 1 & All Saints' Day \\
\hline November 11 & Remembrance Day \\
\hline December 25 & Christmas \\
\hline
\end{tabular}

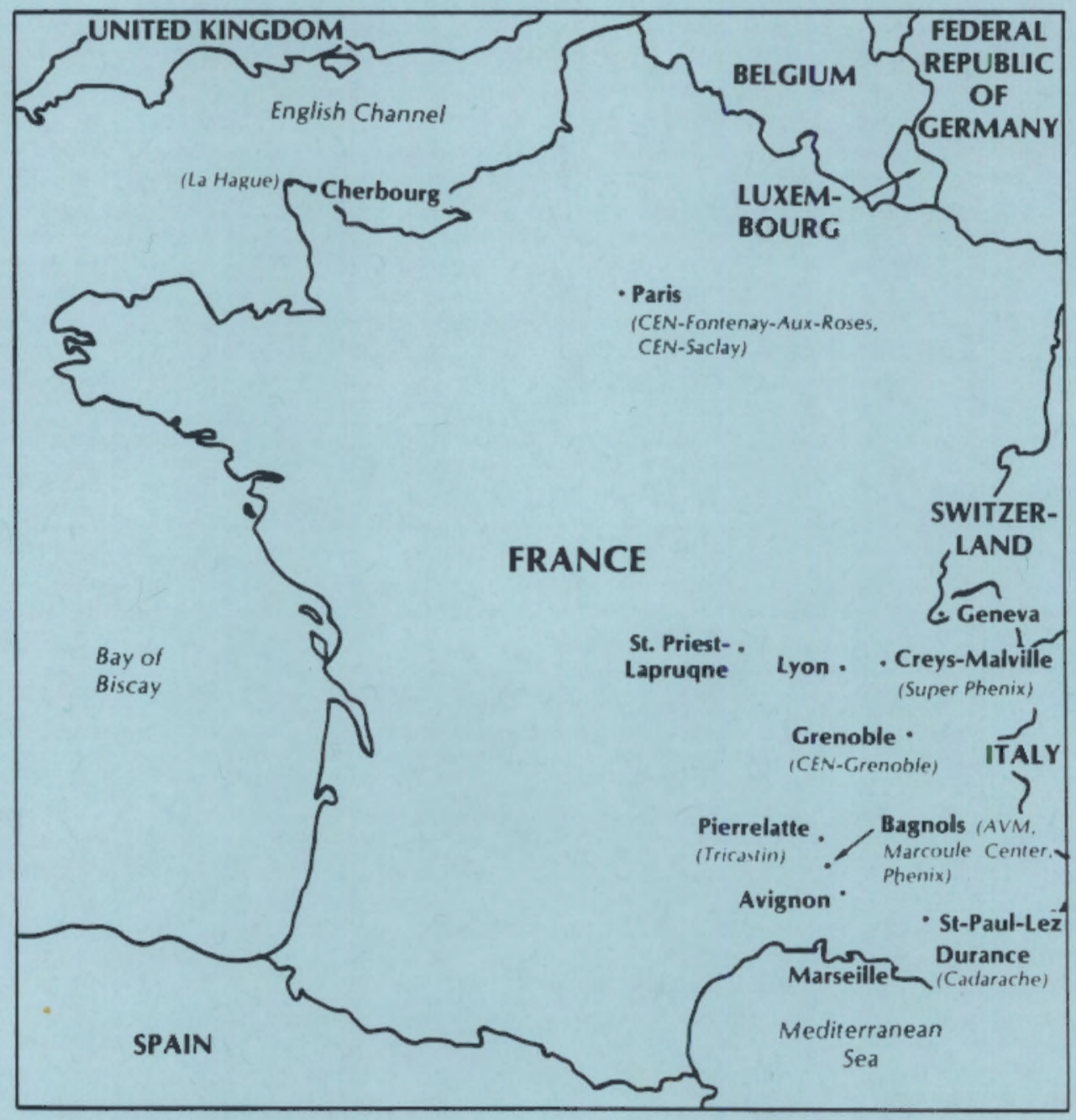


FRANCE

ENERGY

\begin{tabular}{|c|c|c|}
\hline Population & 1981 & 53.8 million \\
\hline Energy Demand & 1980 & $\begin{array}{l}198 \text { Mtoe } \\
\text { ( } 74 \% \text { imported) }\end{array}$ \\
\hline Oil Demand & 1980 & $\begin{array}{l}115 \text { Mtoe } \\
\text { (98\% imported) }\end{array}$ \\
\hline Electric Power Plant Capacity & 1980 & 66 GWe \\
\hline Electric Power Production & 1980 & $\begin{array}{l}258 \text { TWh-- } \\
23.7 \% \text { nuclear } \\
27.4 \% \text { hydro/geo. } \\
25.9 \% \text { solid fuels } \\
23.0 \% \text { oil \& gas } \\
266 \text { TWh-- } \\
38.7 \% \text { nuclear }\end{array}$ \\
\hline
\end{tabular}

NUCLEAR POWER

National Policy. Aggressive nuclear power program, scaled down recently to construction of one or two new reactors per year; commercialization of the breeder reactor; export of nuclear plants and services.

$\begin{array}{lll}\text { Nuclear Power Plant Capacity } & 1983 & 24.6 \text { GWe } \\ & 1985 & 35.6 \text { GWe } \\ & 1990 & 51.0 \text { GWe } \\ & 2000 & 61.2 \text { GWe }\end{array}$

Reactor Mix

1982 GCR: $8(1959-72)$

PWR: $20(1967-82)$

$32(1983-90)$

LMFBR: $2(1973,1985)$

Reactor Development: LMFBR commercialization; Super Phenix--1985; follow-on work still under debate. 
INDUSTRIAL FUEL CYCLE

Policy. Maintain full domestic fuel cycle capability; aggressive export of fuel cycle plants, equipment and services (including uranium enrichment and spent fuel reprocessing).

Waste Management Strategy. HLW--vitrify and store in engineered storage facility for indefinite period, then emplace in geologic repository (granite, salt, clay or seabed). LLW--immobilize in bitumen, concrete or resin and store in surface facility.

\section{Fuel Arisings, tU}

$$
\frac{1980}{248} \quad \frac{1985}{2,700} \quad \frac{1990}{7,700} \quad \frac{2000}{22,000}
$$

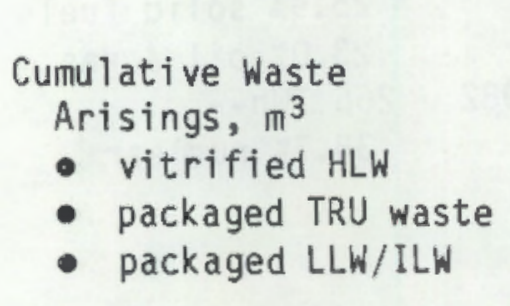

$$
\begin{array}{rrr}
1983 & 1990 & \multicolumn{2}{c}{2000} \\
& & \\
400 & 750 & 3,000 \\
10,000 & 15,000 & 45,000 \\
210,000 & 410,000 & 800,000
\end{array}
$$

Industrial-Scale Activities

1. Uranium mining and milling $(t U)$ : $1981--2,000$; 1985-3,900 .

2. Uranium enrichment ( $t$ SwU/a)

- Pierrelatte, gaseous diffusion: 400-600

- Eurodif, gaseous diffusion: 1982--10,800.

3. Fuel fabrication (tHM/a)

- $\mathrm{UO}_{2}$ : current capacity--400; $1983--600 ; 1986--$ 1,100

- MOX: for FBR fuels--5; for LWR fuels--15.

4. Spent fuel reprocessing

- Marcoule: $3 \mathrm{tU} /$ day, U metal fuels

- La Hague: $3 \mathrm{tU} /$ day, U metal fuels; $0.7 \mathrm{tU} /$ day, $\mathrm{UO}_{2}$ fuels (to be expanded to $5 \mathrm{tHM} /$ day). 
Major Milestones

- TOR demonstration reprocessing plant 1985 for FBR fuels (Marcoule)

- UP3 reprocessing plant (La Hague)

$1988-9$

- UP2-800 reprocessing plant (La Hague) 1990

- Completion of foreign reprocessing 1995 contracts

- AVH waste vitrification plants (La Hague) 1987-9

- New LLW disposal site

- Underground Research Laboratory

INTERNATIONAL RELATIONSHIPS

1. Membership in European Communities, IAEA and OECD/NEA.

2. Major role in international uranium enrichment companies, Eurodif and Coredif.

3. Partnership with DWK (FRG) and BNFL (UK) in United Reprocessors GmbH and in Nuclear Transport, Limited.

4. Leadership of international consortium involved in Super Phenix project.

5. Participation of EdF in SERENA, international FBR technology marketing organization.

\section{ORGANIZATION}

1. Electricité de France, EdF (100\% government)--public power generation; owns and operates all nuclear plants except Phenix and Super Phenix

2. Atomic Energy Commission, CEA--controls all nuclear $R \& D$ and industrial fuel cycle activities

R\&D: Nuclear research centers at Cadarache, Fontenay-aux-Roses, Grenoble, Marcoule, Saclay; Institute for Nuclear Protection and Safety Commercial fuel cycle: COGEMA (mining, uranium conversion, enrichment, reprocessing); SGN, a COGEMA subsidiary (design, construction and marketing of fuel cycle facilities)

Commercial waste disposal: ANDRA. 


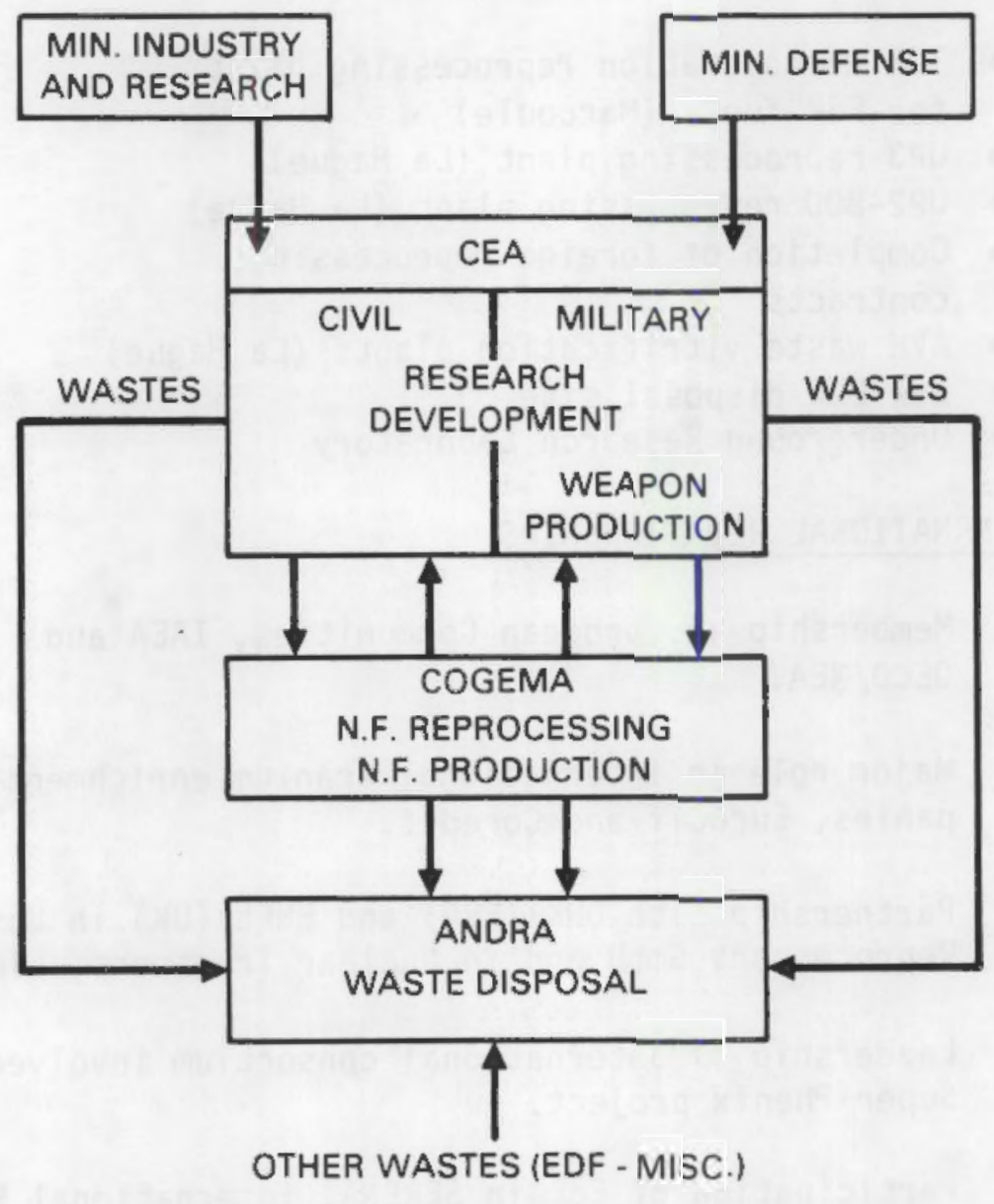

FIGURE FR-1. CEA-COGEMA-ANDRA Activities 


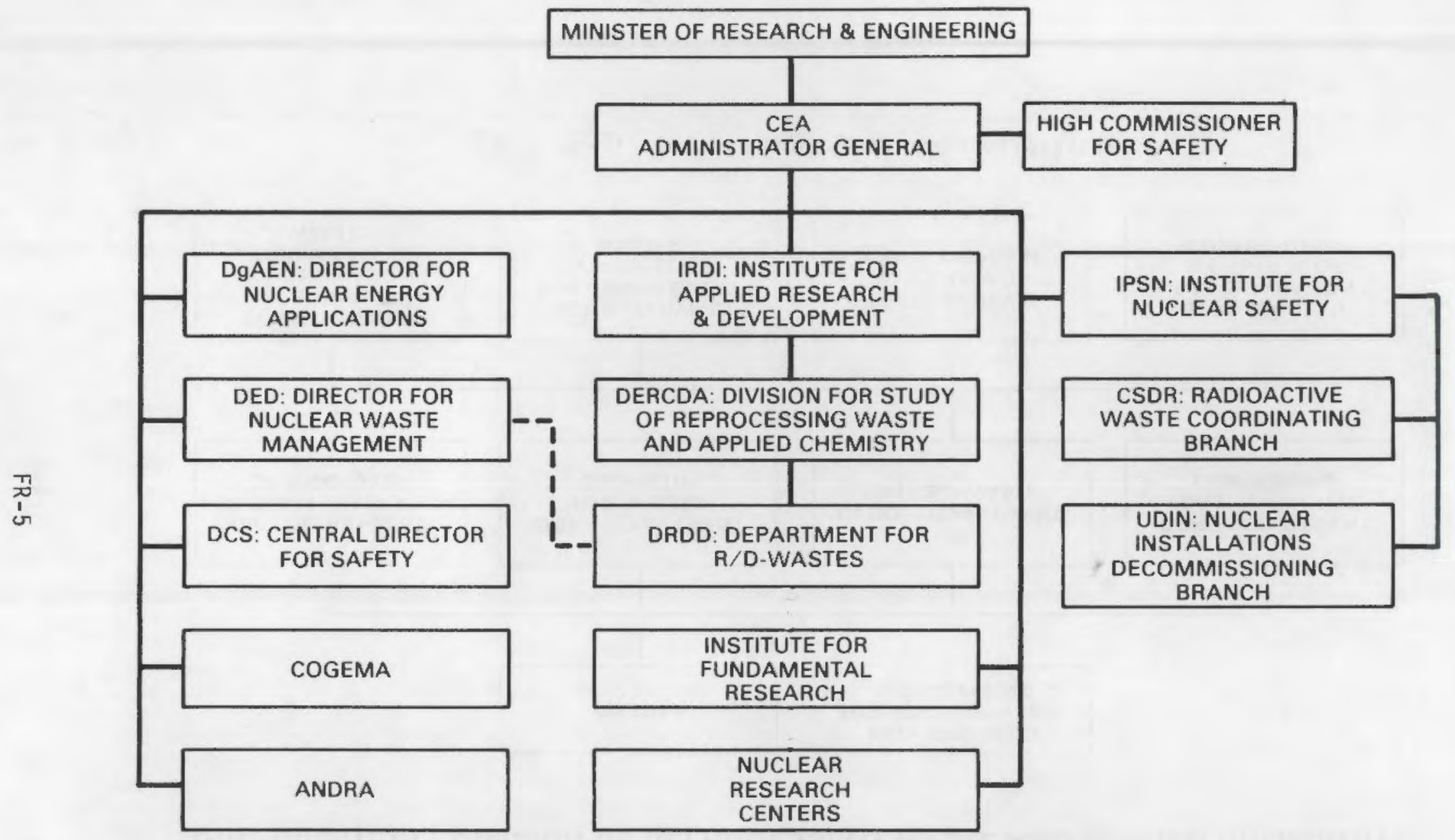

FIGURE FR-2. COGEMA and CEA Research Centers 


\section{ORGANIZATION - DIVISION OF REPROCESSING WASTE AND APPLIED CHEMISTRY}

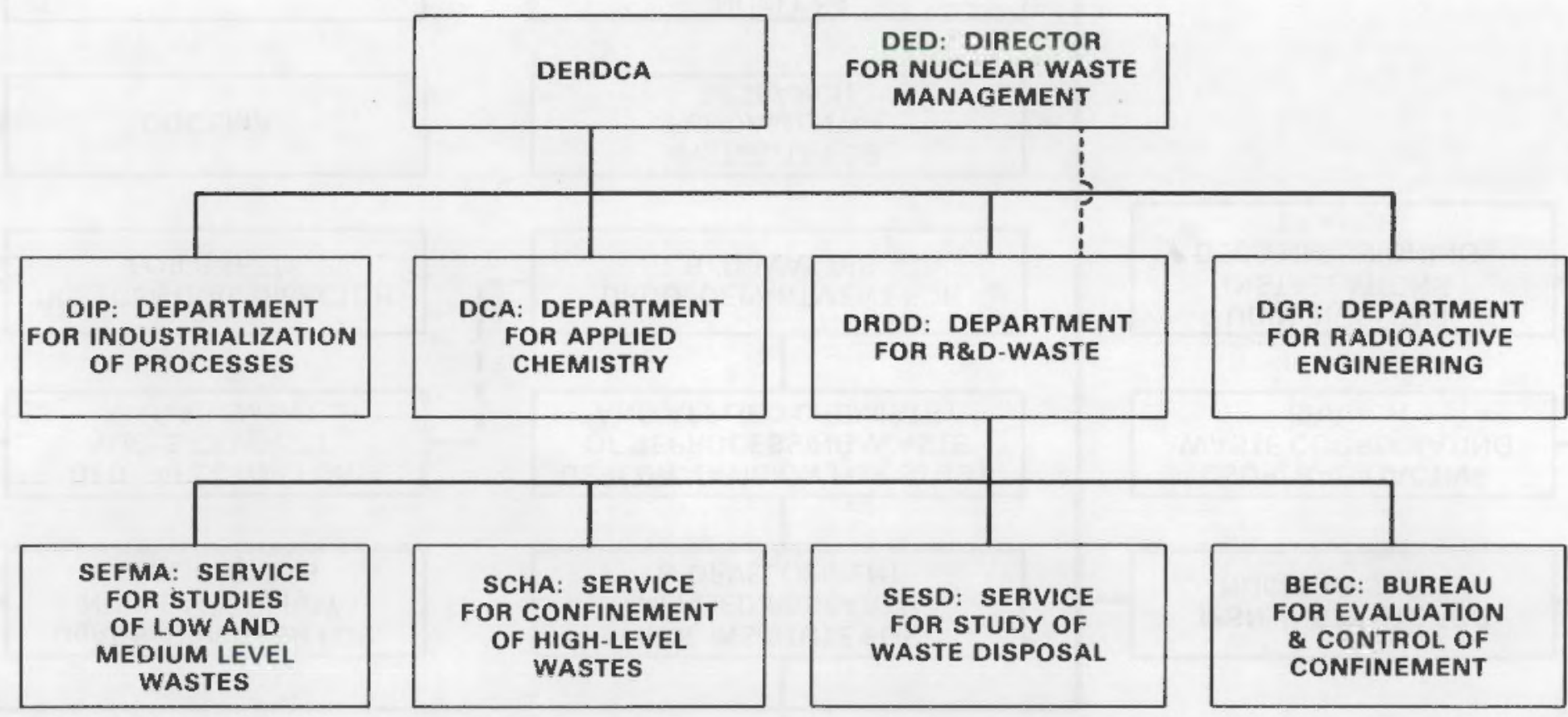

FIGURE FR-3. Nuclear Fuel Cycle Responsibilities 


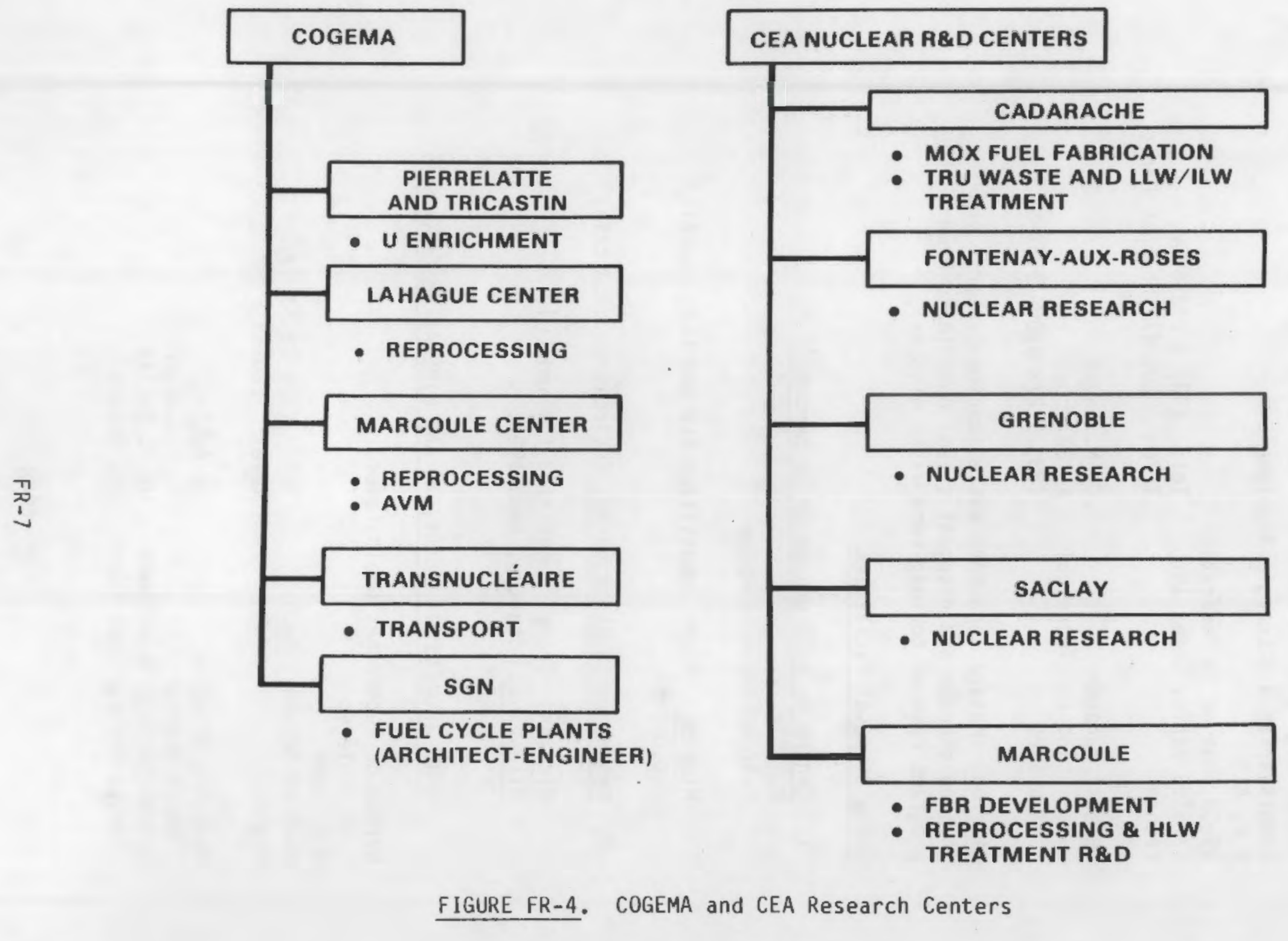


ANDRA (NATIONAL AGENCY FOR RADIOACTIVE WASTE MANAGEMENT)

Agence Nationale pour la Gestion des Déchets

Radioactifs

Commissariat à l'Energie Atomique

B.P. 510

29-33, Rue de la Fédération

F-75752 Paris, Cedex 15

Tel: (33) 12736000 ,

France

Telex: 200671 Energat Paris

General Manager

J. M. Lavie

Assistant for Technical

Matters

A. Barthoux

(33) $12736000-49.60$

Mission. Manage long-term waste storage centers; provide for new storage and disposal sites; coordinate French R\&D programs related to long-term waste disposal.

Waste Disposal Facilities

1. Centre de la Stockage de la Manche (CSM)

B.P. 71

F-50440 Beaumont-Hague

Mission. Store immobilized ILW and LLW; capacity: $300,000 \mathrm{~m}^{3}$.

2. Centre de la Stockage No. 2 (location uncertain)

Mission. Store immobilized ILW and LLW. Milestone. Startup, 1986/1987.

BRGM (BUREAU OF GEOLOGICAL AND MINERAL RESEARCH)

Bureau de Recherches Géologiques

et Minières

B.P. 6009

F-45060 Orleans, Cedex Tel: (33) 38638001

France

Telex: $780258 \mathrm{~F}$

$\begin{array}{ll}\text { Managing Director } & \text { H. Astie } \\ \text { Waste Storage } & \text { P. Peaudecerf } \\ \text { Hydrogeology Department } & \text { J. J. Collin } \\ \text { Geotechnology Department } & \text { Ph. Masure }\end{array}$

$\mathrm{FR}-8$ 


\section{CEA (ATOMIC ENERGY COMMISSION).}

Commissariat a l'Énergie Atomique (CEA)

Centre d'Etudes Nucléaires (CEN)

29-33, Rue de la Fédération

F-75752 Paris, Cedex 15

France

Tel: (33) 12736000

Telex: 200671 Energat

Administrator General

High Commissioner for Safety

Gerard Renon

Director, Nuclear Safety

Director for Nuclear Waste

Management

(Fontenay-aux-Roses)

Assistant Director,

Internationa 1

Assistant Director, R\&D

Director, Safety

Jean Teillac

Pierre Tanguy

Jean Lefevre

(33) 16547471

Pierre Jourde

Annie Sugier

Director, Nuclear Energy

Applications

Jacques Bellot

Michael Rapin

CEA-IRDI (INSTITUTE FOR APPLIED RESEARCH AND DEVELOPMENT)

\begin{tabular}{|c|c|}
\hline $\begin{array}{l}\text { irector, Division, Reprocessing } \\
\text { Waste and Applied Chemistry }\end{array}$ & J. Megy \\
\hline $\begin{array}{l}\text { Director, Department, Indus- } \\
\text { trialization of Processes }\end{array}$ & Courouble \\
\hline $\begin{array}{l}\text { Director, Department, } \\
\text { Radioactive Engineering }\end{array}$ & J. Sauteron \\
\hline $\begin{array}{l}\text { Director, Department, Applied } \\
\text { Chemistry }\end{array}$ & Leveque \\
\hline $\begin{array}{l}\text { Director, Department, R\&D-- } \\
\text { Waste (Fontenay-aux-Roses) }\end{array}$ & $\begin{array}{l}\text { Guy Baudin } \\
\text { Tel: (33) } 16548222 \\
\text { Telex: FAR } 204841\end{array}$ \\
\hline Studies of LLW/ILW (Cadarache) & D. Alexandre \\
\hline $\begin{array}{l}\text { Confinement of High Level } \\
\text { Waste (Marcoule) }\end{array}$ & M. Bonniaud \\
\hline $\begin{array}{l}\text { Waste Disposal Study } \\
\text { (Fontenay-aux-Roses) }\end{array}$ & Jorda \\
\hline $\begin{array}{l}\text { Waste R\&D; Evaluation and } \\
\text { Control of Waste Confine- } \\
\text { ment (Cadarache) }\end{array}$ & $\begin{array}{l}\text { Paul Pottier } \\
\text { Tel: (33) } 42257364 \\
\text { Telex: CEACA } 440678 \mathrm{~F}\end{array}$ \\
\hline
\end{tabular}


CEA-IPSN (INSTITUTE OF PROTECTION AND NUCLEAR SAFETY)

Institut de Protection et de Sûreté Nucléaires (ISPN)

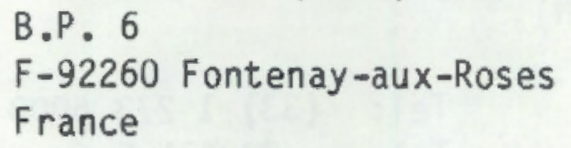

Tel: (33) 16547080

Telex: 204841 Energat Fnayr

P. Tanguy

0. Queniart

J. Pradel

A. M. Chapuis

Bailly

\section{CEA-MARCOULE CENTER}

CEA Etablissement de la Vallée du Rhone

B.P. 171

F-30200 Bagnols-sur-Cèze

Tel: (33) 66895390

Marcoule, France

Telex: $480232 \mathrm{~F}$
Manager, Service for Confinement
M. Bonniaud
of High-Level Wastes
Deputy Manager
C. Sombret
Manager, Laboratory Studies
N. Jacquet-Francillon
Manager, Cold Technology
A. Jouan
Manager, Hot Technology
F. Laude

\section{$\underline{\text { Facilities }}$}

1. SAP (hot pilot plant-reprocessing)

Mission. Develop technology for LWR and LMFBR fuels. Design Basis. PUREX flowsheet, mixer-settlers and pulsed columns; capacity, $10-30 \mathrm{~kg} /$ day (to be increased to $5 \mathrm{tHM} / \mathrm{a}$ with addition of TOR head-end). History. SAP has processed $50 \mathrm{~kg} H M$ from Rapsodie, $2600 \mathrm{~kg}$ HM-uranium from Phenix, and $1210 \mathrm{~kg} \mathrm{HM} \mathrm{(MOX)}$ from Phenix. A new head-end (TOR) is being added to allow reprocessing of $F B R$ fuels. 


\section{CEA-MARCOULE CENTER (contd)}

2. PIVER (hot pilot plant-vitrification)

Mission. Test batch vitrification processes

(1969-1973); produce samples for characterization (1978- ).

Design Basis. Pot calciner/melter; capacity, $90 \mathrm{~kg}$ glass/batch or 25-30 $\mathrm{m}^{3} \mathrm{HLLW} / \mathrm{a}$; product, borosilicate glass blocks, $25 \mathrm{~cm}$ dia by $2.5 \mathrm{~m}$ high.

History. Produced 12 tons glass, 1969-1973. Now used for special process studies.

3. ATLAS (hot pilot plant-vitrification)

Mission. Conduct off-gas treatment studies for AVM process.

Design Basis. AVM: rotary calciner, metallic melter; capacity, 1/2 AVM scale; product, borosilicate glass blocks.

History. Startup, 1978.

4. $\quad$ TOR (reprocessing head-end pilot plant)

Mission. Equip Marcoule SAP pilot plant to reprocess Phenix fuels. Design Basis. $5 \mathrm{tHM} / \mathrm{a}$. Milestones. Startup, 1984-1985.

\section{CEN-CA (CADARACHE NUCLEAR RESEARCH CENTER)}

Centre d'Études Nucléaires de Cadarache

B.P. 1

F-13115 Saint-Paul-lez Durance Tel: (33) 42257000

France

Telex: CEACA $440678 \mathrm{~F}$

(Marseille-Marignane Airport; $65 \mathrm{~km}$ to Cadarache by car provided by Center, or rental car.) 
CEN-CA (CADARACHE NUCLEAR RESEARCH CENTER) (contd)

$\begin{array}{ll}\text { Department of Waste R\&D (D.R.D.D.); } & \text { Paul Pottier } \\ \text { Bureau for Evaluation and } & \text { (33) } 42257364 \\ \text { Control of Waste Confinement } & \\ \text { (B.E.C.C.) } & \text { Y. Francois } \\ \text { Information Center for Nuclear } & \text { (33) } 42257882 \\ \text { Waste } & \text { D. Alexandre } \\ \text { Service for Studies of LLW/ILW } & \text { (33) } 42257123\end{array}$

Fuel Cycle R\&D. Treatment of TRU waste, LLW, and ILW; properties of non-HLW waste forms; MOX fuel fabrication; and waste isolation (radionuclide migration).

Facilities

1. Solid Waste Treatment Pilot Plant

Mission. Develop methods to handle solid wastes (cryogenic crushing and ultrasonic washing). Design Capacity. Eight 200-1b drums/hr. History. Startup, 1984.

2. Bituminization Plant

Design Basis. Immobilize reactor wastes; twin-screw extruder; capacity, $260 \mathrm{~m}^{3} / \mathrm{a}$.

History. Startup, 1977.

3. MOX Fuel Fabrication

Mission. Fabricate $\mathrm{PuO}_{2}-\mathrm{UO}_{2}$ fuels for FBRs and for recycle to LWRs. Design Capacity. $15 \mathrm{t} / \mathrm{a}$, LWR fuels; $5 \mathrm{t} / \mathrm{a}$, FBR fuels.

4. LLW Incinerator

5. Solvent Incinerator 


\section{CEN-FaR (FONTENAY-AUX-ROSES NUCLEAR RESEARCH CENTER)}

Centre d'Études Nucléaires

de Fontenay-aux-Roses

B.P. 6

F-92260 Fontenay-aux-Roses

France

(See CEA-IRDI Institute for Applied R\&D)

Waste Management R\&D. Safety-related research; airborne waste treatment; solvent extraction processes for waste partitioning; OKLO studies.

\section{CEN-G (GRENOBLE NUCLEAR RESEARCH CENTER)}

Centre d'Études Nucléaires

de Grenoble

Avenue des Martyrs $85 \mathrm{X}$

F-38041 Grenoble, Cedex

France

Director

Tel: (33) 76974111

Telex: 320323 Energat

Greno

Pierre Corbet

Waste Management R\&D Treatment of reactor wastes and removal of tritium from heavy water used as a reactor moderator.

\section{CEN-S (SACLAY NUCLEAR RESEARCH CENTER)}

Centre d'Études Nucléaires

de Saclay

F-91191 Gif-sur-Yvette, Cedex

France

Te1: (33) 69086000

Telex: $690641 \mathrm{~F}$ Energat

Saclay

Director

Paul Mirat

Nuclear Wastes

Henri Vialettes 


\section{CEN-S (SACLAY NUCLEAR RESEARCH CENTER) (contd)}

Facilities

Bituminization Plant (radioactive)

Mission. Immobilize non-HLW.

Design Basis. Turbulent film evaporator with heating jacket and internal rotor.

CFC (COGEMA FRAMATOME COMBUSTIBLE)

Location. Under construction at Pierrelatte.

Sponsors. Framatome and Cogema.

Facilities

CFC Fuel Plant

Mission. $\mathrm{UO}_{2}$ fuel fabrication for EdF. Design Capacity. $500 \mathrm{t} / \mathrm{a}$. Milestone. Startup, 1983.

COGEMA (COMPAGNIE GÉNÉRALE DES MATIERES NUCLÉAIRES)

COGEMA, Inc.

Direction Générale

B.P. 4

F-78141 Velizy Villacoublay Cedex Tel: (33) 39469641

France

Telex: COGEM 697833

President

Manager, Reprocessing Plants

Francois de Wissoca

Industrial Director, Reprocessing Plants

$U_{3}$ Reprocessing Plant

Claude Aycoberry

Maurice Delange

Francois Chenevier 
COGEMA-LA HAGUE CENTER

COGEMA, Centre de La Hague

B.P. 270

F-50107 Cherbourg

Tel: (33) 33036000

France

Telex: 170030 Energat Hag

Director

Jean Parmentier

Reprocessing Department

Waste Management

Jean-Louis Ricaud

Mr. Laurent

Fuel Cycle Program. Spent fuel reprocessing and HLW vitrification. The La Hague plant was originally designed to handle magnesium-clad $U$ metal fuels from gas/graphite power reactors. Current plans are to transfer all gas/ graphite fuels reprocessing to Marcoule UP-1 and devote La Hague to treating LWR fuels with occasional FBR fuel campaigns through UP-2.

\section{Facilities}

1. UP2 (fuel reprocessing plant)

Mission. Reprocess magnesium-clad, natural uranium metal fuels from gas/graphite reactors and oxide fuels from LWRs and Phenix FBR. (Phenix fuel is diluted with natural uranium fuel for criticality control.)

Design Basis. Metal fuels: mechanical decladding; PUREX flowsheet; capacity, 800 t/a and 1.6 tPu/a; contact maintenance. FBR and LWR oxide fuels: shear-leach HAO head-end; remote maintenance; capacity, $1.3 \mathrm{t} /$ day of LWR fuels. The nominal capacity was reduced to $250 \mathrm{t} / \mathrm{a}$ in 1980 , when one dissolver was taken out of service. HAO expansion to $800 \mathrm{t} / \mathrm{a}$ of LWR fuels started in late 1980 , to be completed in early 1990s.

History. Startup: UP-2, 1967; HAO, 1976. During 1967-1977, annual throughputs of gas/graphite reactor fuels reportedly averaged 220-270 tHM. 1980, subsequent throughputs: $253 \mathrm{tHM}$ from gas/graphite fuels, $105 \mathrm{t}$ from LWRs and $1.5 \mathrm{t}$ from Phenix; 1981, $101.3 \mathrm{t}$ from LWRs and Phenix; January 1-June 24, 1982, 153 t LWR fuel. Total HAO throughput from startup through $6 / 80=280 \mathrm{t}$ LWR fuel. 
COGEMA-LA HAGUE CENTER (contd)

2. UP3 (fuel reprocessing plant)

Mission. Reprocess French and foreign LWR fuels. Design Basis. Chop-leach head-end; PUREX flowsheet; capacity, 4 t/day each for UP-3A and UP-3B. Milestones. Startup, UP3--1988/9.

3. AT-1 (hot pilot plant-reprocessing)

Mission. Test technology, reprocess FBR fuels. Design Basis. Chop-leach head-end and mixersettlers; capacity, $1 \mathrm{~kg} \mathrm{HM} /$ day. History. Hot startup, 1972. Total throughput by $1979=1.1$ tHM from FBR fuel.

4. AVH (vitrification plant)

Mission. Vitrify La Hague HLW. Design Basis. AVM process: rotary calciner, metallic or ceramic melter; capacity, $600 \mathrm{~m}^{3} / \mathrm{a}$ HLLW (three 1 ines of $50 \mathrm{l} / \mathrm{hr}$ HLLW, $25 \mathrm{~kg} / \mathrm{hr}$ glass). Canister dimensions: $42 \mathrm{~cm}$ dia $\times 1.3 \mathrm{~m} \mathrm{high} \mathrm{(400} \mathrm{kg} \mathrm{glass).}$ Milestone. Startup, mid-1986.

\section{COGEMA-MARCOULE CENTER}

COGEMA

Centre de Marcoule

B.P. 170

F-30200 Bagnols-sur-Cèze Tel: (33) 66895390

Marcoule, France

Telex: 480232F MARCOGEMA

(Marseille-Marignane Airport; by train to Avignon; car provided by Center.)

Director Reprocessing Plant.

Maurice Allès

Maurice Chot in 


\section{COGEMA-MARCOULE CENTER (contd)}

\section{Facilities}

1. UP1 (reprocessing plant)

Mission. Reprocess magnesium-clad natural uranium metal fuels from military or gas/graphite power reactors.

Design Basis. Mechanical declad; PUREX flowsheet; contact maintenance; capacity, 4.5-6 tHM/day. Expansion is under way to allow treatment of 400-450 thM/a of gas/graphite reactor fuel, in addition to military fuel load.

History. Startup, 1958; 280 tHM of gas/graphite fuel processed in 1959, in addition to military fuels. Reportedly, total 1958-1981 throughput was about 12,000 tHM.

2. PURR (prototype d'usine de retraitement des rapids)

Mission. Reprocess FBR (MOX) fuels. Design Capacity. 50-100 thM/a. Milestone. Construction deferred, pending government decision on future of FBR program.

3. AVM (ateliers de vitrification de Marcoule)

Mission. Demonstrate AVM process: vitrify Marcoule UP-1 wastes. Design Basis. Rotary calciner feeding an inductionheated metallic melter; capacity, $30 \mathrm{l} / \mathrm{hr}$ HLLW feed and $360 \mathrm{~kg} /$ day (1 canister) borosilicate glass product; waste form, glass blocks $0.5 \mathrm{~m}$ dia $\times 1.0 \mathrm{~m}$ high. History. Hot startup, $6 / 78$; as of $7 / 1 / 82,546 \mathrm{~m}^{3}$ of HLLW from reprocessing 9657 tonnes of fuel had been vitrified to yield 251 tonnes of glass (734 canisters). Activity in the waste totaled $88 \times 10^{6} \mathrm{Ci}$. As of $3 / 31 / 83,623 \mathrm{~m}^{3}$ of HLLW had been treated. 
COGEMA-MARCOULE CENTER (contd)

4. Incinerator

Mission. Volume reduction of combustible wastes. Design Basis. Triple chamber, propane-heated; capacity, $60-70 \mathrm{~kg} / \mathrm{hr}$ solids.

5. Bituminization Facility

Mission. Immobilize LLW and ILW. Design Basis. Continuous screw extruder; capacity, $100 \mathrm{~kg} / \mathrm{hr}$. History. Startup, 1976.

\section{DAM (DIRECTORATE OF MILITARY APPLICATIONS)}

Direction des Applications Militaires

Commissariat a 1 'Energie Atomique

31-33 Rue de la Fédération

F-75752 Paris, Cedex 15

France

FBFC (FRANCO-BELGE COMPANY FOR FUEL FABRICATION)

Société Franco-Belge de Fabrication de Combustibles

Avenue Bertie Albrecht

F-75008 Paris

Te1: (33) 17665200

France

Telex: 290503

Facilities

1. Fuel Fabrication Plant (Romans, France)

Mission. Fabricate $\mathrm{UO}_{2}$ fuels for power reactors. Design Capacity. $400 \mathrm{t} / \mathrm{a}$ (to be increased to $600 \mathrm{t} / \mathrm{a})$.

2. Fuel Fabrication Plant (Dessel, Belgium)

Mission. Fabricate $\mathrm{UO}_{2}$ fuels. Design Capacity. $400 \mathrm{t} / \mathrm{a}$. 
PARIS SCHOOL OF MINES

Ecole Natínale Supérieure des Mines de Paris Centre d'Informatique Géologique

35 Rue Saint-Honoré

F-77305 Fontainebleau

Te1: (33) 64224821

France

Telex: 600736 MINEFON

Director, Mathematical Geology Dr. Ghislain de Marsily Center

Deputy Director

Dr. Emmanuel Ledoux

Research Engineers Dr. Patrick Goblet

Dr. Michel Poulin

Dr. Anne Ribstein

Waste Management R\&D. Geologic waste isolation (fluid flow, heat transport and mass transport studies--theoretical, laboratory and field tests).

SGN (SOCIÉTÉ GÉNÉRALE POUR LES TECHNIQUES NOUVELLES)

Société Générale pour les

Techniques Nouvelles.

1 Rue des Hérons, Montigny le Bretonneux

F-78184 Saint Quentin Yvelines, Tel: (33) 10586000

Cedex, France

Telex: $698316 \mathrm{~F}$

President

Louis Pradere

Managing Director

Technical Director

Pierre Boutaud de la Combe

Claude Bernard

Operations Vice President

Jean-Pierre Giraud

International Projects

Jacques Merlet

International Relations

Michel Lung

\section{TN (TRANSNUCLÉAIRE)}

Transnucléaire

11 Rue Christophe-Colomb

F-75008 Paris

France

Tel: (33) 17237850

Telex: 280992

General Manager

Armand Aupetit

Technical Manager

Paul Blum

FR-19 


\section{France}

\section{US EMBASSY - PARIS}

US Embassy

2 Avenue Gabriel

75382 Paris CEDEX 08

France

Science Counselor
Tel: (33) 1 296-1202

Telex: 650221

J. Boright 


\section{GERMANY (FRG)}




\begin{tabular}{|c|c|}
\hline January 1 & New Year's Day \\
\hline *March/April & Good Friday \\
\hline *March/April & Easter Monday \\
\hline May 1 & Labor Day \\
\hline *May/June & Ascension Day \\
\hline $\begin{array}{l}\text { *May/June } \\
\text { June } 17\end{array}$ & $\begin{array}{l}\text { Whit Monday } \\
\text { Day of Unity }\end{array}$ \\
\hline *November & Repentence Day \\
\hline December 25-26 & Christmas \\
\hline
\end{tabular}

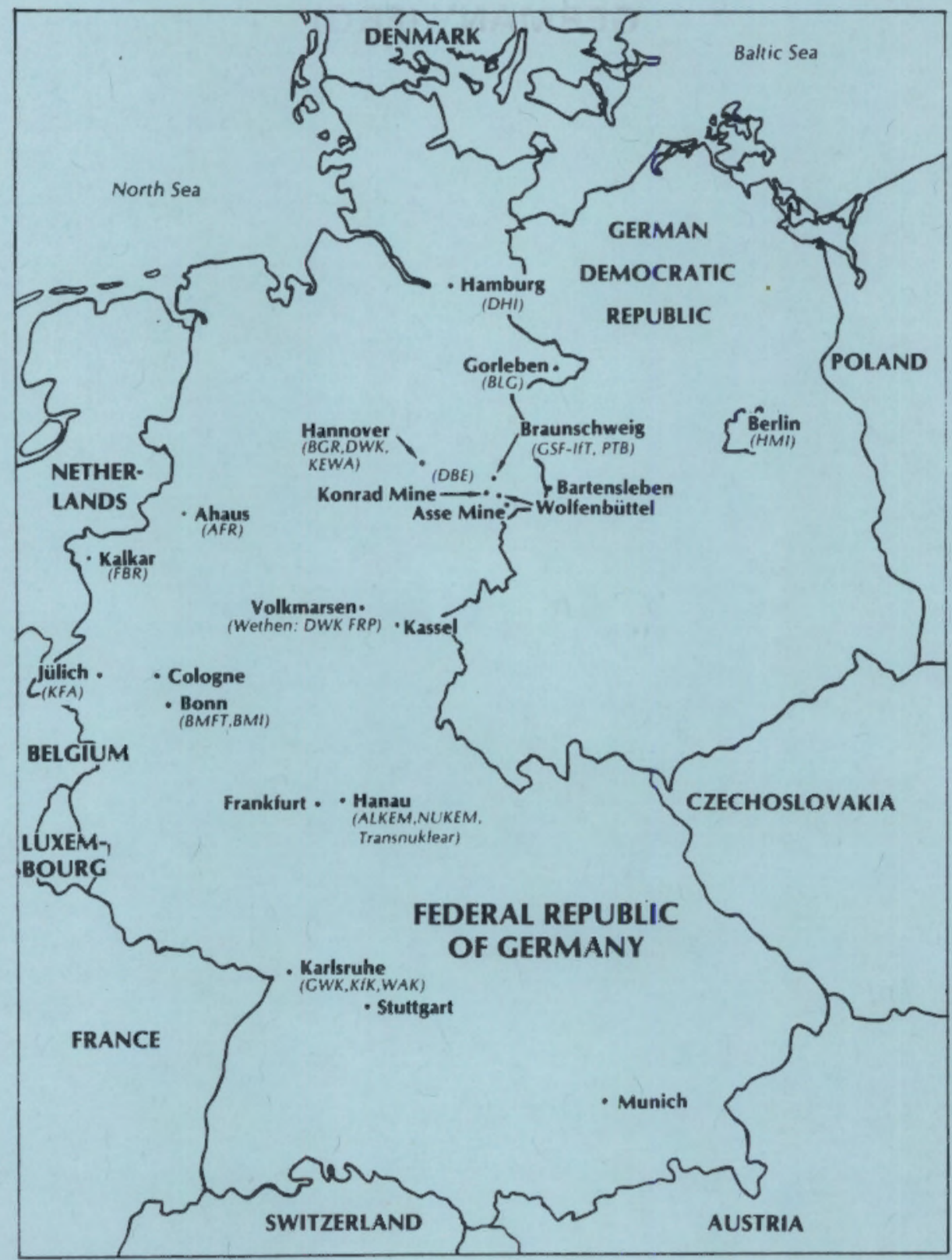




\section{GERMANY (FRG)}

\section{ENERGY}

Population

Energy Demand

0i1 Demand

Electric Power Plant Capacity

Electric Power Production
198061.6 million

1980272 Mtoe

(55\% imported)

1982257 Mtoe

1980133 Mtoe ( $96 \%$ imported)

$1980 \quad 86$ GWe

1980348 TWh--

$11.9 \%$ nuclear

$55.3 \%$ solid fuels

$26.4 \%$ oi 1 /gas

$6.4 \%$ hydro/other

$198217 \%$ nuclear

$198525 \%$ nuclear

1990 25-30\% nuclear

NUCLEAR POWER

National Policy: strong support for private-enterprise nuclear industry; federal funding of nuclear research; joint financing (federal/industry) of demonstration projects.

$\begin{array}{lrr}\text { Nuclear Power Plant Capacity } & 1983 & 9.8 \text { GWe } \\ 1985 & 16.4 \text { GWe } \\ 1990 & 22.9 \text { GWe } \\ & 2000 & 28.8 \text { GWe } \\ & 1982 & \text { PWR: } 8(1972-82) \text {; } \\ & & 10(1983-91) \\ \text { Reactor Mix } & & \text { BWR: } 5(1972-80) \text {; } \\ & & 3(1983-85)\end{array}$


INDUSTRIAL FUEL CYCLE

Policy. Full commercial capability (private companies)-enrichment; fuel fabrication; domestic reprocessing of standard LWR fuels; plutonium recycle to FBRs and LWRs. Immediate reprocessing load is to be handled by foreign plants. The federal government provides and operates waste disposal facilities; the power producers pay for the service.

Waste Management Strategy. Vitrification of HLW and interim storage of HLW glass for 20-30 years; disposal of HLW glass, TRU and possibly spent fuels in salt-dome repository; geologic disposal of other radioactive wastes in salt repository or abandoned iron mine.

Cumulative Spent Fuel Arisings (LWR)

Cumulative Waste Arisings

$$
\begin{array}{lr}
1980 & 960 \mathrm{tU} \\
1985 & 2,250 \mathrm{tU} \\
1990 & 4,550 \mathrm{tU} \\
2000 & 11,000 \mathrm{tU} \\
2000 & 250-500,000 \text { drums } \\
& \text { LLW; 70-100,000 } \\
& \text { drums ILW; 4,600 } \\
& \text { blocks HLW glass }
\end{array}
$$

Industrial-Scale Activities

1. Uranium mining and milling (tU/a): 100.

2. Uranium enrichment (tSWU/a): 1986--400.

3. Conversion of $\mathrm{UO}_{2}$ to UF $6(\mathrm{tU} / \mathrm{a}): 1000$.

4. Fuel fabrication

- $\mathrm{UO}_{2}$ fuels: $950 \mathrm{tU} / \mathrm{a}$

- MOX fuel: $40 \mathrm{tHM} / \mathrm{a}$ for LWR fuels, $10 \mathrm{thM} / \mathrm{a}$ for FBR fuel elements (ALKEM).

5. AFR spent fuel storage

- 1,500 t, dry storage (Anaus; demonstration license obtained)

- 1,500 t, dry storage (Gorleben; startup, 1983).

6. Construction of $350 \mathrm{t} / \mathrm{a}$ fuel reprocessing plant (DWK--Bavaria and/or Lower Saxony). 
Major Milestones

- Complete site characterization at Gorleben

- Policy decision between domestic

After 1985 fuel reprocessing and spent fuel disposal

- Spent fuel AFR at Gorleben

- First fuel reprocessing plant

- PAMELA HLW vitrification pilot plant

- HOVA HLW vitrification plant for the WAK pilot plant

- Konrad iron mine repository

- Gorleben repository

\section{INTERNATIONAL RELATIONSHIPS}

1. FRG is a member state of OECD/NEA, IAEA and the European Communities.

2. Bilateral agreement (BMFT/DOE) for waste management cooperation.

3. Commercial ties with other countries:

- URENCO, the UK-Netherlands-FRG uranium enrichment consortium

- United Reprocessors (UNIREP), the UK-France-FRG reprocessing consortium

- Nuclear Transport, Limited (NTL), with France and the UK

- Partnership with Belgium and The Netherlands in SNR-300 (FRG)

- Five percent interest in Super Phenix (France)

- Thirty-five percent interest in SERENA, FRG-France marketing agency

- Nuclear technology transfer to Brazil

- Cooperation with General Atomic in HTGR development

- Licensing arrangements with US reactor vendors. 
ORGANIZATION

1. Federal Government

- Coordinates FRG nuclear program

- Sponsors R\&D

- Builds and operates radioactive waste disposal facilities.

2. Privately-owned Utilities

- Provide spent fuel storage, reprocessing and waste treatment

- Pay for waste disposal.

3. States (Länder)

- License nuclear plants. 

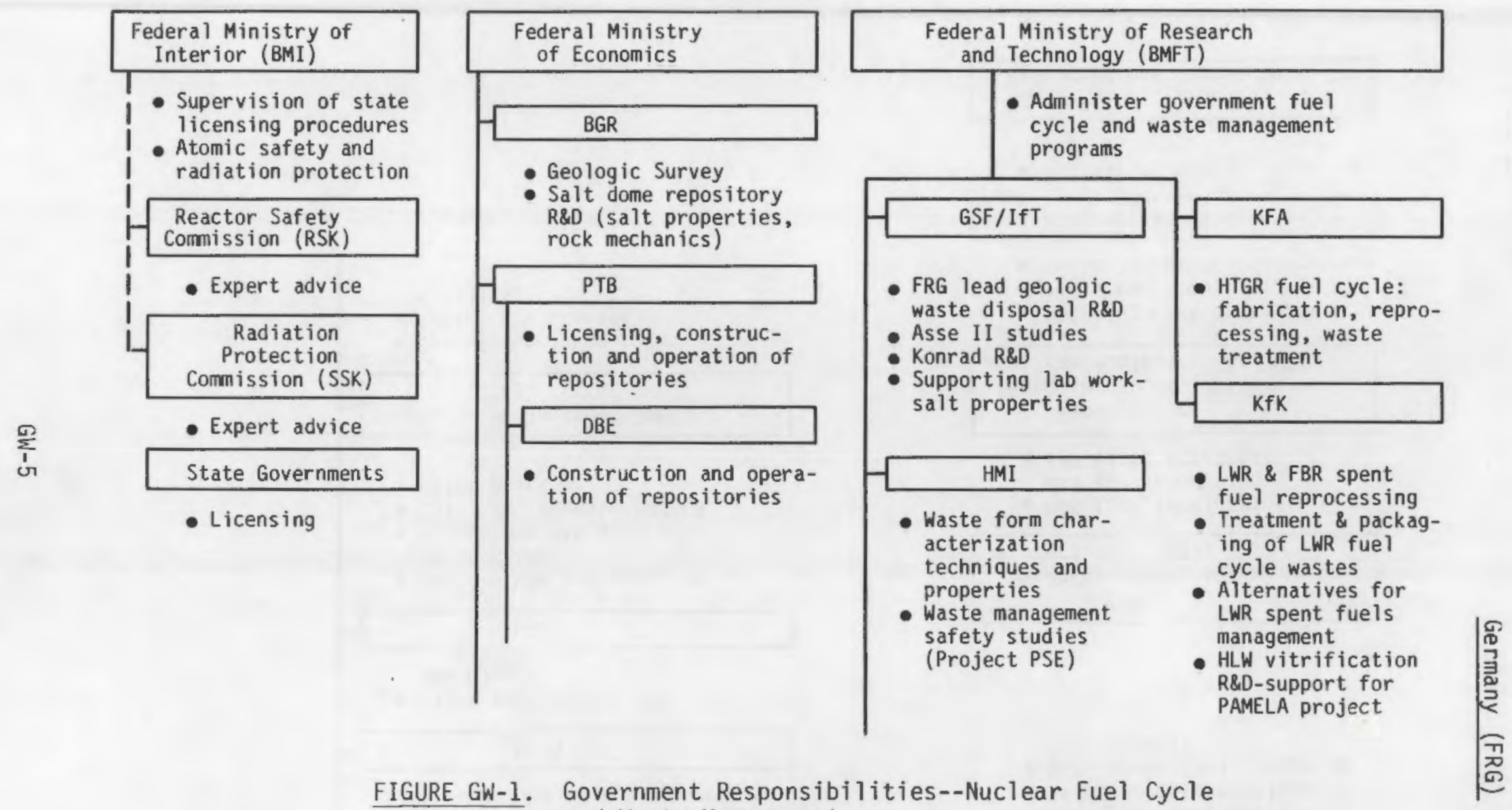

FIGURE GW-1. Government Responsibilities--Nuclear Fuel Cycle and Waste Management 
DWK

(Owned by FRG Nuclear Utilities)

- Provide fuel reprocessing and waste conditioning services for FRG utilities

- Finance waste disposal services provided by federal government

\section{KEWA}

- Plant engineering for DWk plants

WAK

- Operate WAK reprocessing pilot plant

- Manage DWK R\&D programs

- Build and operate PAMELA

pilot plant

Brennelement Gorleben GmbH (BLG)

- Operate Gorleben spent fuel \& LLW storage facilities
NUKEM

(Owned by DeGussa, 45\%;

RWE, 25\%; RTZ, 18\%; MG, 12\%)

- LLW \& TRU waste treatment R\&D \& facility design

- Shipping cask development

- R\&D--spent fuel packaging for disposal

ALKEM
(Owned by NUKEM, 40\%
and KWU, 60\%)

- MOX fuel fabrication

R\&D and production

- TRU waste treatment

Transnuk lear

(Owned by NUKEM, $80 \%$ and Transnucléaire SA, 20\%

- Shipping cask development and production

- Portable LLW solidification

\begin{tabular}{|l|}
\hline Berlin Technical University \\
\hline Repository shaft \\
sealing R\&D \\
\hline $\begin{array}{c}\text { University of Munich, } \\
\text { Radiochemical Institute }\end{array}$ \\
\hline
\end{tabular}

- Actinide chemistry R\&D

FIGURE GW-2. Industrial and University Responsibilities--Nuclear Fuel Cycle and Waste Management Activities 


\section{ALKEM}

ALKEM GmbH

Postfach 110069

D-6450 Hanau 11 (Wolfgang)

Tel: (49) $618158-0$

Federal Republic of Germany

Telex: 04184124 rbu d

Company Directors

Horst Roepenack

(49) $618158-4600$

Dr. Wolfgang Stoll

(49) $618158-4666$

Plutonium Waste Treatment

Dr. Volker Schneider

(49) $618158-4590$

Dr. F.-W. Ledebrink

(49) $618158-4155$

Facilities

MoX Fuel Fabrication Plant

Capacity. $40 \mathrm{t} / \mathrm{a}$, LWR fuel; $10 \mathrm{t} / \mathrm{a}$, FBR fuel.

BAM (FEDERAL INSTITUTE FOR MATERIAL TESTING)

Bundesanstalt für Material Prüfung (BAM)

Unter den Eichen 87

D-1000 Berlin 45

Tel: (49) 30 8104-1

Federal Republic of Germany Telex: 183261 BAMB D

BGR (FEDERAL INSTITUTE FOR GEOSCIENCES

AND NATURAL RESOURCES)

Bundesanstalt für Geowissenschaften und

Rohst of fe

Postfach 510153

D-3000 Hannover 51

Tel: (49) $5116468-1$

Federal Republic of Germany Telex: $923730 \mathrm{bfb}$ 
BGR (FEDERAL INSTITUTE FOR GEOSCIENCES

AND NATURAL RESOURCES) (contd)
Director, Division 2, General and Technical Geology Director, Subdivision, Engineering Geology
Rock Mechanics
Soil Mechanics
Salt Mechanics
Rock Mechanics in Mining and Technical Research
Disposal of Radioactive Waste
Underground Storage
Hydrogeology
Groundwater Geophysics

\author{
Prof. Helmut Venzlaff \\ Prof. M. Langer \\ Prof. A. Pahl \\ Dr. R. Lüdeling \\ Dr. H. Albrecht \\ Dr. D. Meister \\ Dr. W. Jaritz \\ Dr. H. Reum \\ Dr. H. Vierhuff \\ Dr. W. Giesel
}

\section{BMFT (FEDERAL MINISTRY FOR SCIENCE AND TECHNOLOGY)}

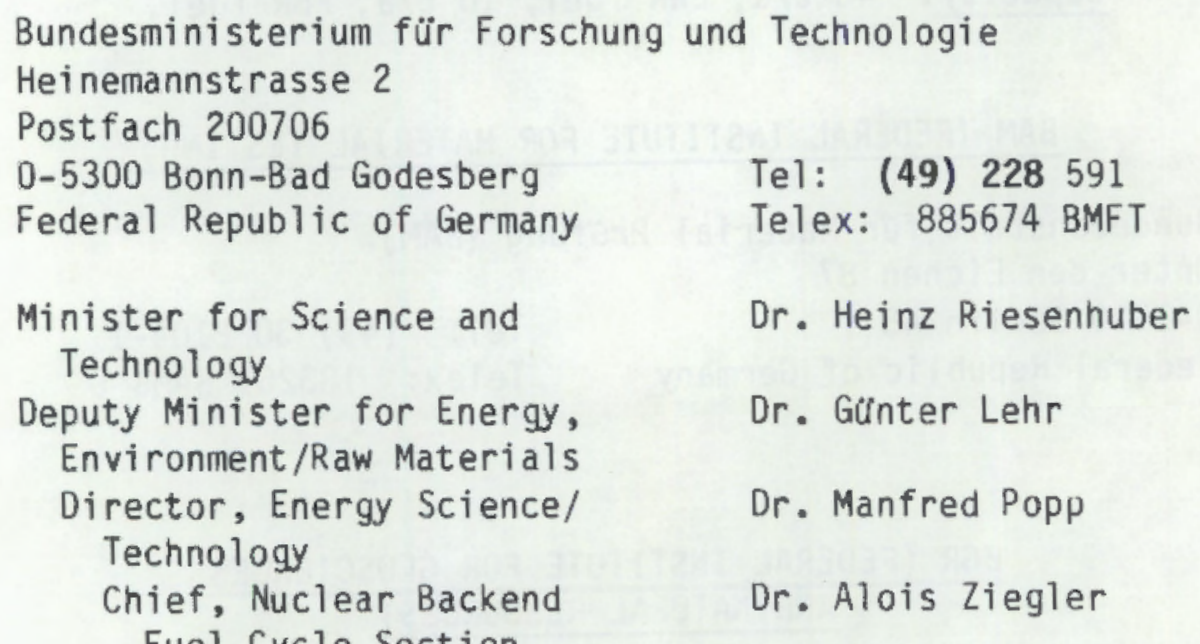




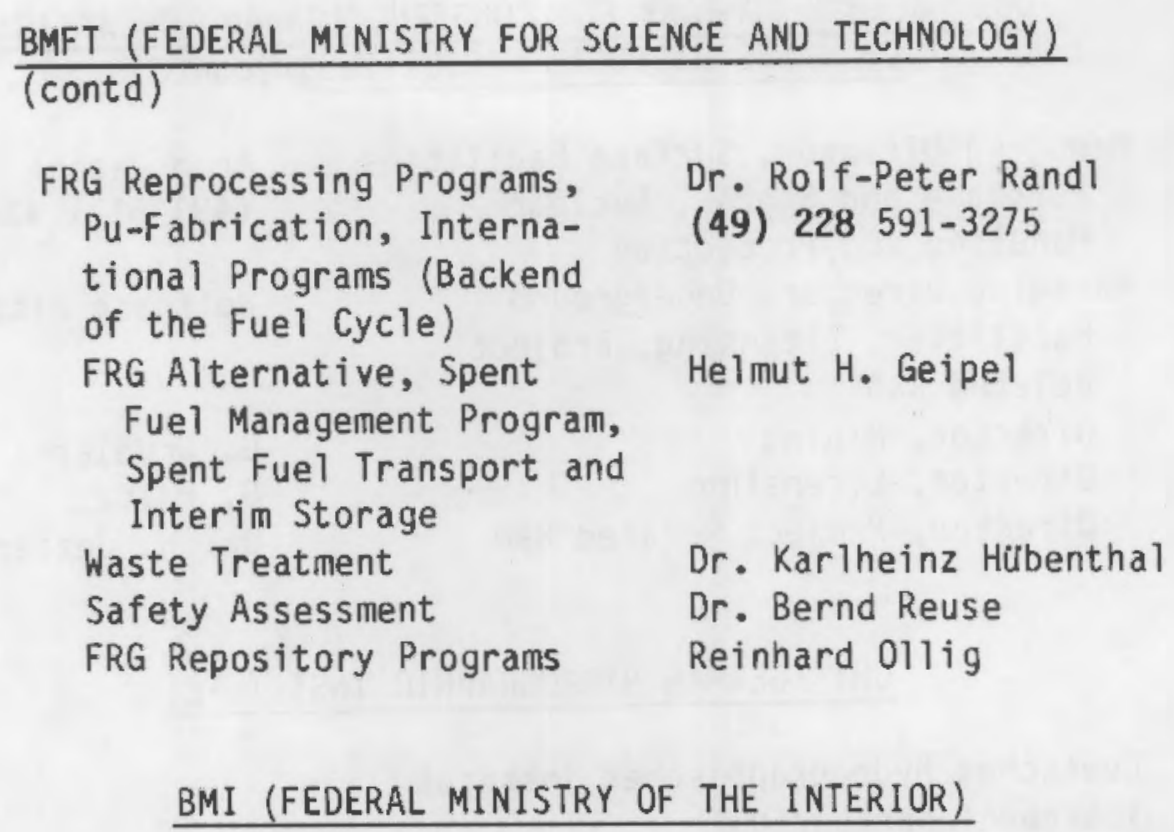

Bundesministerium des Innern

Graurheindorfer Strasse 198

D-5300 Bonn 1

Federal Republic of Germany

Chairman, Advisory Committee on Reactor Safeguards (RSK) Chairman, Advisory Committee on Radiological Protection (SSK)

D. Smidt

A. Kaul

DBE (GERMAN COMPANY FOR CONSTRUCTION AND OPERATION OF WASTE DISPOSAL FACILITIES)

Deutsche Gesellschaft zum Bau und Betrieb von Endlagern für Abfallstoffe mbH (DBE) Woltorfer Strasse 74

D-3150 Peine, 1

Tel: (49) 5171431

Federal Republic of Germany

Telex: $092646 \mathrm{dbe} d$ 
DBE (GERMAN COMPANY FOR CONSTRUCTION AND OPERATION OF WASTE DISPOSAL FACILITIES) (contd)

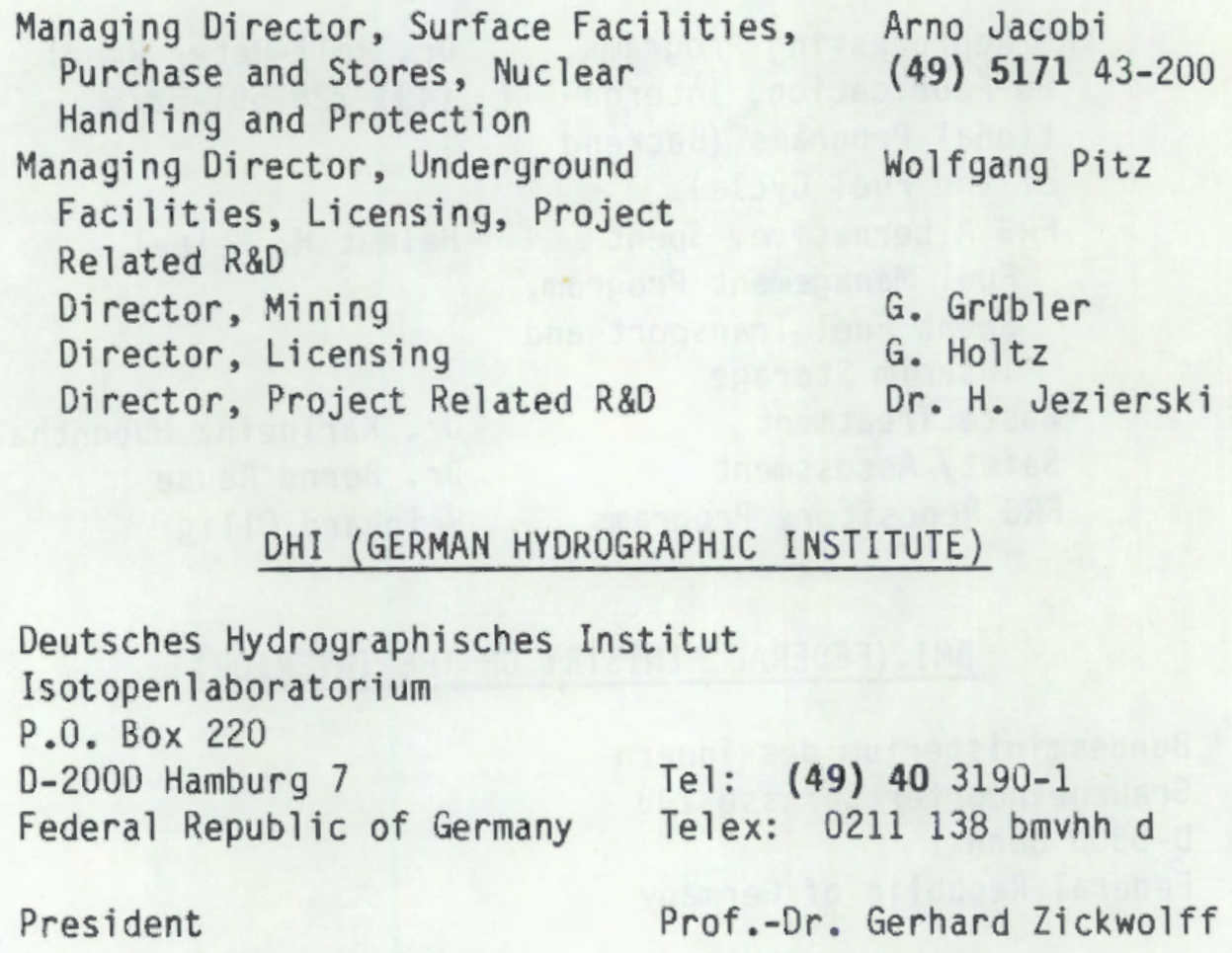


Germany (FRG)

DWK (KEWA DIVISION)

KEWA Kernbrennst of $f$-Wiederaufarbeitungs-GmbH

Hamburger Allee 4, Postfach 1407 Tel: (49) 51133901

D-3000 Hannover 1

Telex: $9222202 \mathrm{dwk}$

Federal Republic of Germany

( für kewa)

Company Directors

Dipl.-Ing. Joachim Mischke

Director, Process Engineering

Dr. H.-J. Mylord

Engineering and Technology

Mr. W. A. Issel

Dr. Knoch

Facilities

1. AFR Spent Fuel Storage Facilities

(Gorleben and Ahaus sites)

Design Basis. Dry storage in CASTOR casks -

400 casks in a building which has dimensions of

$600 \mathrm{ft} \times 125 \mathrm{ft} \times 62 \mathrm{ft}$ high; capacity, $1500 \mathrm{t}$.

Milestone. Startup of Gorleben AFR, 1983.

2. Fuel Reprocessing Plant (Bavaria or Lower Saxony)

Mission. Demonstrate operability and safety of small commercial plant; reprocess spent LWR fuels commercially.

Design Basis. Shear-leach head-end; PUREX process;

capacity, $350 \mathrm{t} / \mathrm{a}$.

Milestone. Startup, 1990.

3. PAMELA Pilot Plant (Mol, Belgium)

Mission. Demonstrate ceramic melter and VITROMET

production with stored Eurochemic LEWC.

Design Basis. Denitration; liquid-fed ceramic

melter, $0 . \overline{72} \mathrm{~m}^{2}$ surface area; capacity, $36-\mathrm{l} / \mathrm{hr}$ feed, $25 \mathrm{~kg} / \mathrm{hr}$ glass (3 canisters/day $150 \mathrm{~kg}$ glass/

canister); product, either borosilicate glass blocks or VITROMET, $0.3 \mathrm{~m}$ dia by $1.2 \mathrm{~m}$ high.

Milestone. Hot operation, 1985. 


\section{GSF/IfT (INSTITUTE FOR UNDERGROUND STORAGE, COMPANY}

FOR RADIATION AND ENVIRONMENTAL RESEARCH)

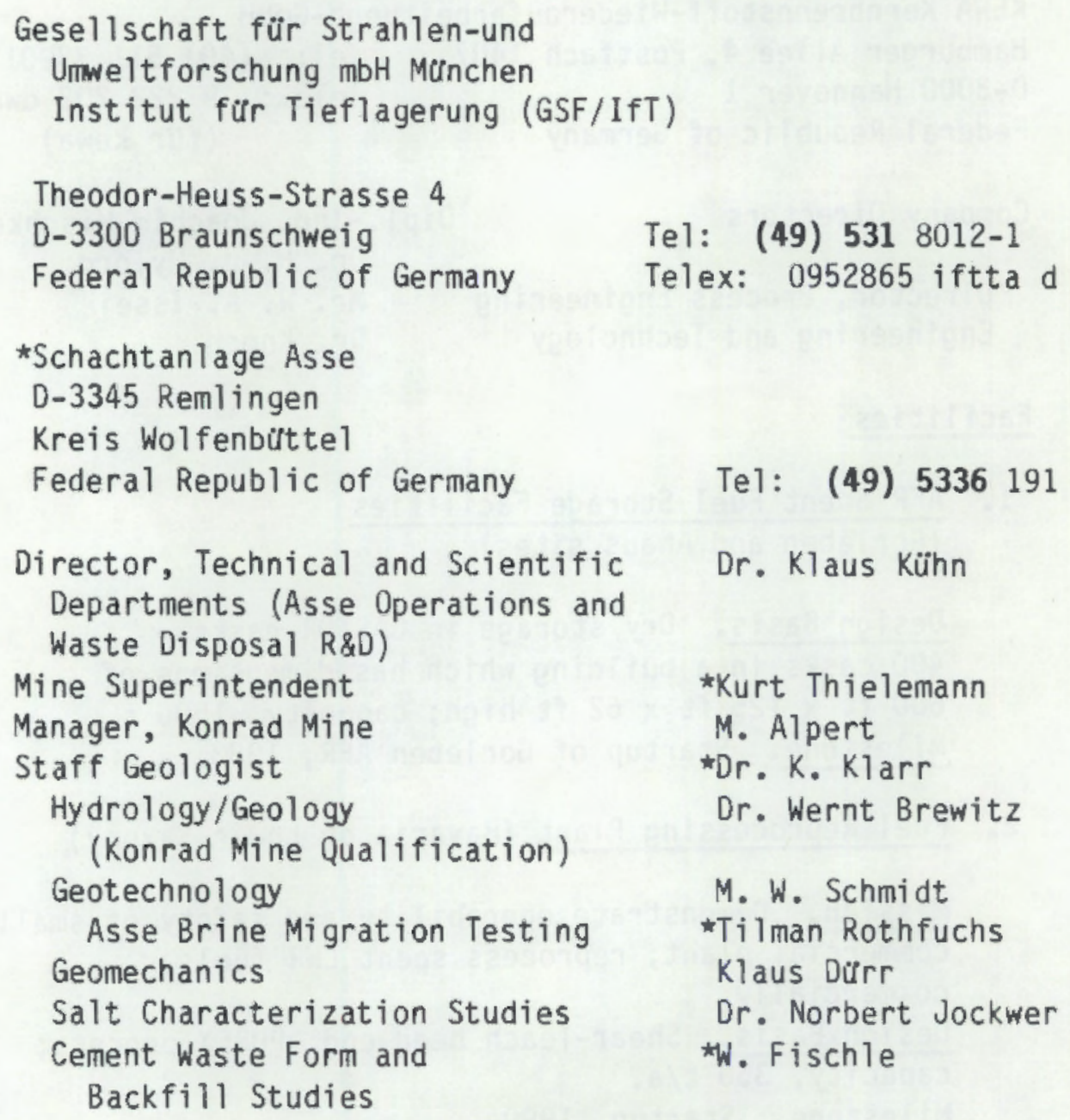

*Kurt Thielemann

M. Alpert

\#Dr. K. K1arr

Dr. Wernt Brewitz

M. W. Schmidt

*Tilman Rothfuchs

Klaus Dürr

Dr. Norbert Jockwer

*W. Fischle

\section{Facilities}

1. Asse II Salt Mine (10 km S.E. of Wolfenbüttel)

Mission. In situ testing and disposal technology development for a salt dome repository; through 1978, disposal of LLW and ILW. History. Startup, 1967.

2. Konrad Iron Mine (10 km S.W. of Braunschweig)

Mission. Disposal of non-TRU LLW; R\&D on hard-rock disposa1. Milestone. Startup as disposal site, 1988. 
GSF/IfT (INSTITUTE FOR UNDERGROUND STORAGE, COMPANY FOR RADIATION AND ENVIRONMENTAL RESEARCH) (contd)

3. Chemical and Hydrology Laboratories (Braunschweig) Mission. Provide supporting services for Asse Mine in-situ testing.

4. Rock Mechanics Laboratory (Braunschweig)

Mission. Rock mechanics R\&D for GSF/IfT waste disposal studies.

\section{HMI (HAHN-MEITNER INSTITUTE)}

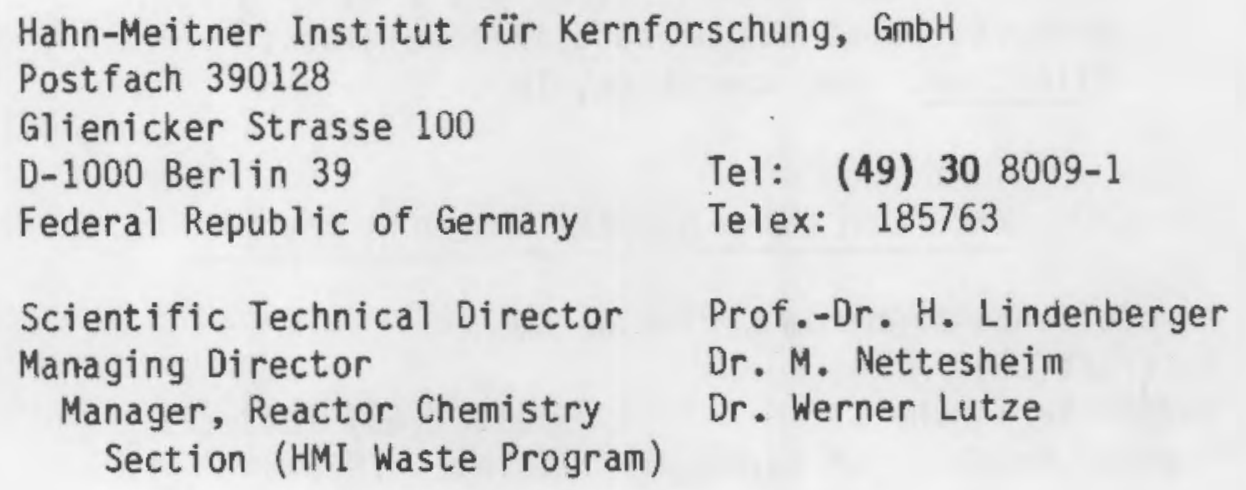

\section{KFA (JÜLICH NUCLEAR RESEARCH CENTER)}

Kernforschungsanlage Jülich GmbH

Postfach 1913

D-5170 Jülich

Federal Republic of Germany

Tel: (49) 2461610

Director, Institute of

Telex: 833556

Chemical Technology

Prof. Erich Merz

Reprocessing and Final

Prof. Erich Zimmer Waste Storage

HTGR Fuel Cycle Project/

G. Kaiser

JUPITER Project

Director, Decontamination

Dr. Manfred Laser

Institute

Director, Institute for Prof. H. Nickel

Reactor Materials 
KFA (JÜLICH NUCLEAR RESEARCH CENTER) (contd)

$\underline{\text { Facilities }}$

1. Jupiter Pilot Plant (radioactive)

Mission. Develop HTGR fuel reprocessing technology. Design Basis. Grind-burn-leach head-end; Thorex solvent extraction. Capacity, $2 \mathrm{~kg} /$ day. Milestone. Startup, 1985.

2. FIPS II (radioactive)

Mission. Develop vitrification technology for HTGR fuel cycle HLW. Design Basis. Denitration to form sludge, drum dryer, in-can melter. Capacity, $1 \mathrm{~kg} / \mathrm{hr}$ glass; product, phosphate or borosilicate glass. Milestone. Hot operation, TBD.

KfK (KARLSRUHE NUCLEAR RESEARCH CENTER)

Kernforschungszentrum Karlsruhe GmbH Postfach 3640

D-7500 Karlsruhe $1 \quad$ Tel: (49) 7247821

Federal Republic of Germany Telex: 7826484

(Most convenient way from US is to fly to Frankfurt and go by train or car to Karlsruhe.)

$\begin{array}{ll}\begin{array}{l}\text { Manager, Fuel Reprocessing and } \\ \text { Wr. Reinhard Kroebel } \\ \text { Project (PWA) }\end{array} & \text { (49) 7247 82-2032 } \\ \text { Director, Waste Treatment } & \text { Dr. Gunter Hơlein } \\ \text { Department (HDB) } & \\ \begin{array}{l}\text { Director, Institute for Hot } \\ \text { Chemistry }\end{array} & \text { Prof. Klaus Ebert } \\ \text { Deputy Director } & \text { Dr. Gunter Koch }\end{array}$


KFK (KARLSRUHE NUCLEAR RESEARCH CENTER) (contd)

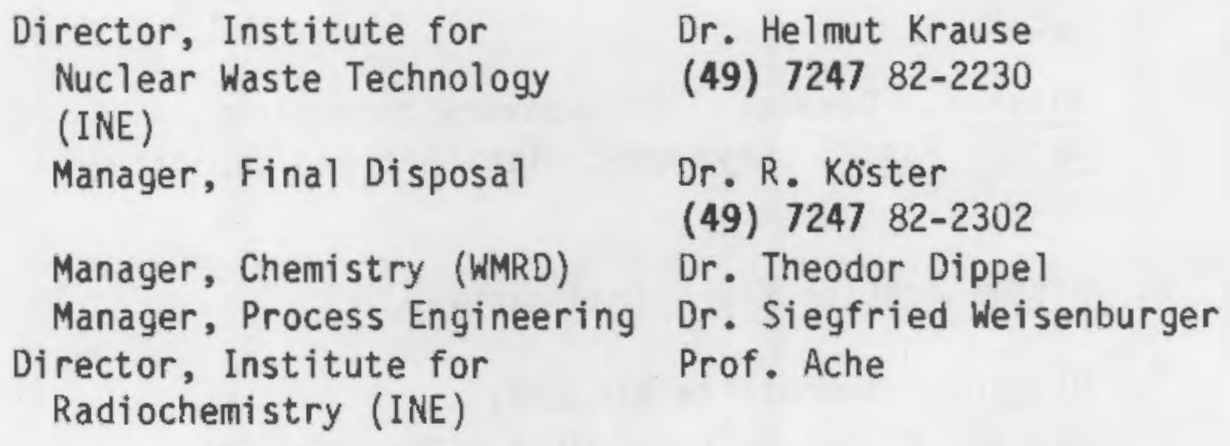

\section{Facilities}

1. Ceramic Melter (nonradioactive)

Mission. HLW vitrification process development, to support PAMELA pilot plant design and operation. Design Basis. Liquid-fed joule heated melter; capacity, $30 \mathrm{l} / \mathrm{hr}$ HLLW or $20 \mathrm{~kg} / \mathrm{hr}$ glass. History. Startup, May 1980.

2. MILLI Hot Cell Facility (fuel reprocessing)

Mission. LWR and FBR fuel reprocessing R\&D.

3. Ceramic Melter (nonradioactive)

Mission. Test remote operation of a ceramic melter facility which is a prototype of the PAMELA hot cell. Design Capacity. $30 \mathrm{\ell} / \mathrm{hr}$ HLLW.

History. Startup, Fall 1981. As of $5 / 83$, had produced $5+$ glass beads and $30+$ blocks, and had demonstrated $90 \%$ availability.

4. ALONA (radioactive acid digestion pilot plant built and operated by KfK at Mol, Belgium)

Mission. Demonstrate acid digestion of combustible TRU wastes. Design Basis. Treatment with $\mathrm{H}_{2} \mathrm{SO}_{4}-\mathrm{HNO}_{3}$ in thermal loop system. Capacity, $1000 \mathrm{~m}^{3} / \mathrm{a}$. History. Startup, 1982. 
KfK (KARLSRUHE NUCLEAR RESEARCH CENTER) (contd)

5. KRETA

Mission. Develop ${ }^{85} \mathrm{Kr}$ recovery technology. Design Basis. Cryogenic distillation; capacity, $50 \mathrm{~m}^{3} / \mathrm{hr}$.

6. Bituminization Plant (radioactive)

Mission. Immobilize KfK LLW.

Design Basis. Self-cleaning screw-extruder-

evaporator.

History. Startup, 1972.

7. Waste Concreting Plant (radioactive)

Mission. Immobilize KfK ILW.

Design Capacity. $2.5 \mathrm{t} /$ day waste.

History. Startup, 1977.

\section{MUNICH TECHNICAL UNIVERSITY}

Technische Universităt München

Walther-Meissner-Strasse

8046 Garching bei Munchen

Te1: (49) 8932092201

Federal Republic of Germany Telex: 522854 tumue d

Director, Institute for Prof. Dr. Franz Baumgärtner

Radiochemistry

NUKEM

NUKEM GmbH

Postfach 110080

D-6450 Hanau 11

Tel: (49) $618158-0$

Federal Republic of Germany Telex: 4184113 nuk d

Managing Directors $P$. Jelinek-Fink

Division Managers, H. Pirk

Process Engineering H. Dyroff

Department Manager, R\&D Dr. H. Huschka 
NUKEM (contd)

$\underline{\text { Facilities }}$

Pyrohydrolysis Incinerator

Mission. Volume reduction of TRU wastes.

Design Capacity. $25 \mathrm{~kg} / \mathrm{hr}$.

History. Startup, 1980.

PTB (FEDERAL SCIENCE AND ENGINEERING LABORATORY)

Physikalisch-Technische Bundesantalt

Bundesallee 100

Postfach 3345

0-3300 Braunschweig

Tel: (49) 531 592-1

Federal Republic of Germany

Telex: 952822 ptb $d$

Director, Division Storage/

Prof. H. Röthemeyer

Disposal of Radioactive

Wastes

(49) $531592-7620$

Subdivision Science/Technology

(Waste Disposal)

Waste Product

Repository Technical Mine Safety

Radiation Safety

R\&D, Accident Analysis

Explorations, Construction

of Mines

Director, Subdivision Fuel

Materials

Transport Licensing

Interim Storage Facilities

Licensing

Dr. E. Warnecke

Dr. P. Desterle

(49) $531 \quad 592-7635$

Dr. Ehrlich

Dr. Illi

G. Wosnick

Dr. W. Collin

M. B1echschmidt (acting)

M. Blechschmidt 
Facilities

Gorleben Disposal Site

Mission. Dispose of high-level and TRU wastes. Repository Concept. 65-m deep boreholes in tunnel floors at depths of $800-1000 \mathrm{~m}$ in the Gorleben salt dome.

Milestone. Startup, 1995.

\section{TRANSNUKLEAR}

Transnuklear GmbH

Postfach 110030

D-6450 Hanau 11

Federal Republic of Germany

Te1: (49) $6181500-1$

Feral Repubtic of Germany

Telex: 4184123 tnf $d$

Company Executives

Dr. Peter Vygen

Dr. Horst Keese

UHDE

Unde GmbH

Friedrich-Uhde-Strasse 15

Postfach 262

D-4600 Dortmund 1

Tel: (49) $231547-0$

Federal Republic of Germany

Telex: $822841-25$ ud $d$

Nuclear and Electrochemical

Dr. Bernd Strasser

Division

Nuclear Division

Mr. Karl-Heinz Knickel

Processing Engineering

Dr. Wolfgang Strewe

Department

\section{US EMBASSY - BONN}

American Embassy

Delchmannsaue

5300 Bonn 2

Federal Republic of Germany

Tel: (49) $228 \quad 339-3390$

Telex: 885452 


\section{WAK GmbH (FUEL REPROCESSING COMPANY)}

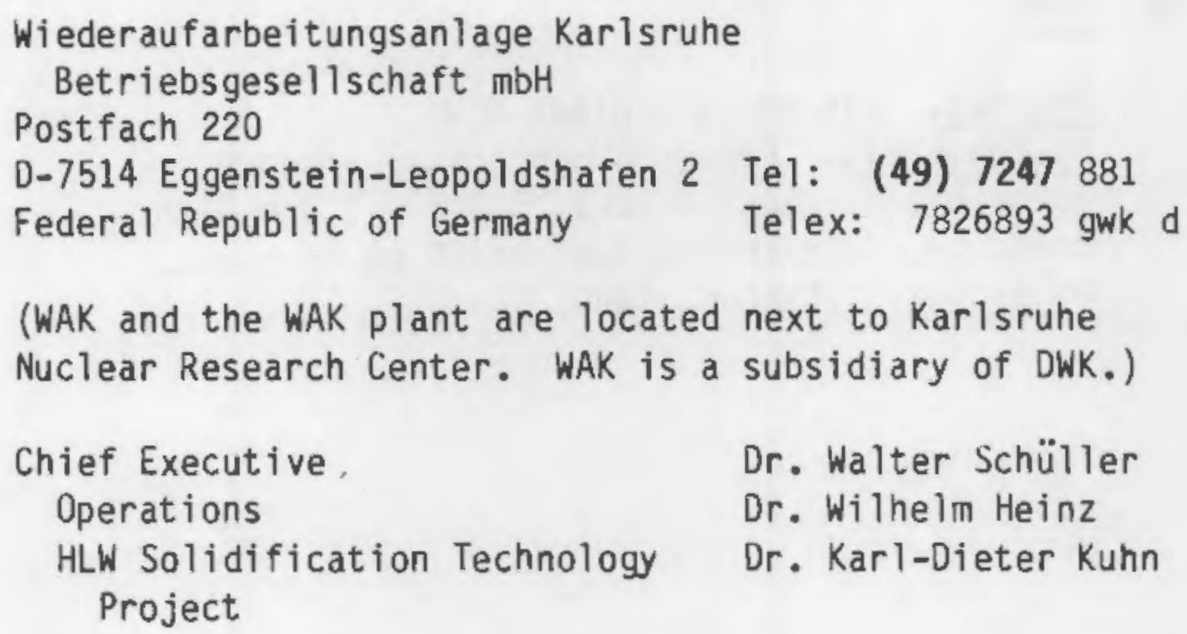

\section{Facilities}

1. WAK Reprocessing Plant (owned by KfK)

Mission. Reprocess $\mathrm{UO}_{2}$ fuels; recover plutonium for recycle; test advanced technology.

Design Basis. Chop-leach head-end; PUREX process; capacity, $175 \mathrm{~kg} \mathrm{HM} /$ day.

History. On-line from $9 / 71$ to early 1980 , when it was shutdown for dissolver replacement. Total throughput to 1980, 115 tHM (43 tHM from LWR fuel). Operation resumed, October 1982.

2. TEKO Hall (cold semi-works, owned by KfK)

Mission. Test fuel cycle components and unit operations; currently being equipped for fuel reprocessing studies.

Design Basis. Shear, dissolver, centrifuge, solvent extraction battery; head-end capacity, 4 thM/day. Milestones. Beneficial occupancy for uranium testing of solvent extraction system--late 1981; installation of head-end components--1982, 1983.

3. $A Z U R$

Mission. Demonstrate dissolver off-gas treatment. Design Basis. Varied; includes cryogenic recovery of ${ }^{85} \mathrm{Kr}$. History. Startup, late 1970s. 
WAK GmbH (FUEL REPROCESSING COMPANY) (contd)

4. HOVA

Mission. Vitrify WAK plant HLW. Design Basis. French AVM process; product, borosilicate glass blocks, $0.3 \mathrm{~m} \times 1.3 \mathrm{~m}$ high; capacity, $30 \mathrm{l} / \mathrm{hr}$ HLW feed or $15 \mathrm{~kg} / \mathrm{hr}$ glass. Milestone. Startup, 1990. 
INDIA 


\section{INDIAN HOLIDAYS}

*Asterisks denote movable holidays

$\begin{array}{cl}\text { January 1 } & \text { New Year's Day } \\ \text { *January } & \text { Id-ul-Fitr } \\ \text { January 26 } & \text { Republic Day } \\ \text { *February } & \text { Vasanta } \\ \text { *February } & \text { Maha Sivarati } \\ \text { *March } & \text { Holi } \\ \text { *March } & \text { Dulhendi } \\ \text { *April } & \text { Durga Ashtmi } \\ \text { *April } & \text { Muharram } \\ \text { *April } & \text { Mahavir Jayanti } \\ \text { *March/April } & \text { Good Friday } \\ \text { April 13 } & \text { Baisakhi } \\ \text { *August } & \text { Raksha Bandhan } \\ \text { August 15 } & \text { Independence Day }\end{array}$

*August

*September

*0ctober

October 2

$\star$ October

*October

*November

December 25

*December

December 31

Janmasht ami

Anant Choudas

Dusehra

Mahatma Gandhi's

Birthday

Diwali

Bhaiya Dooj

Guru Nanak's Birthday

Christmas

Guru Gobind

Singh's Birthday

Bank Holiday

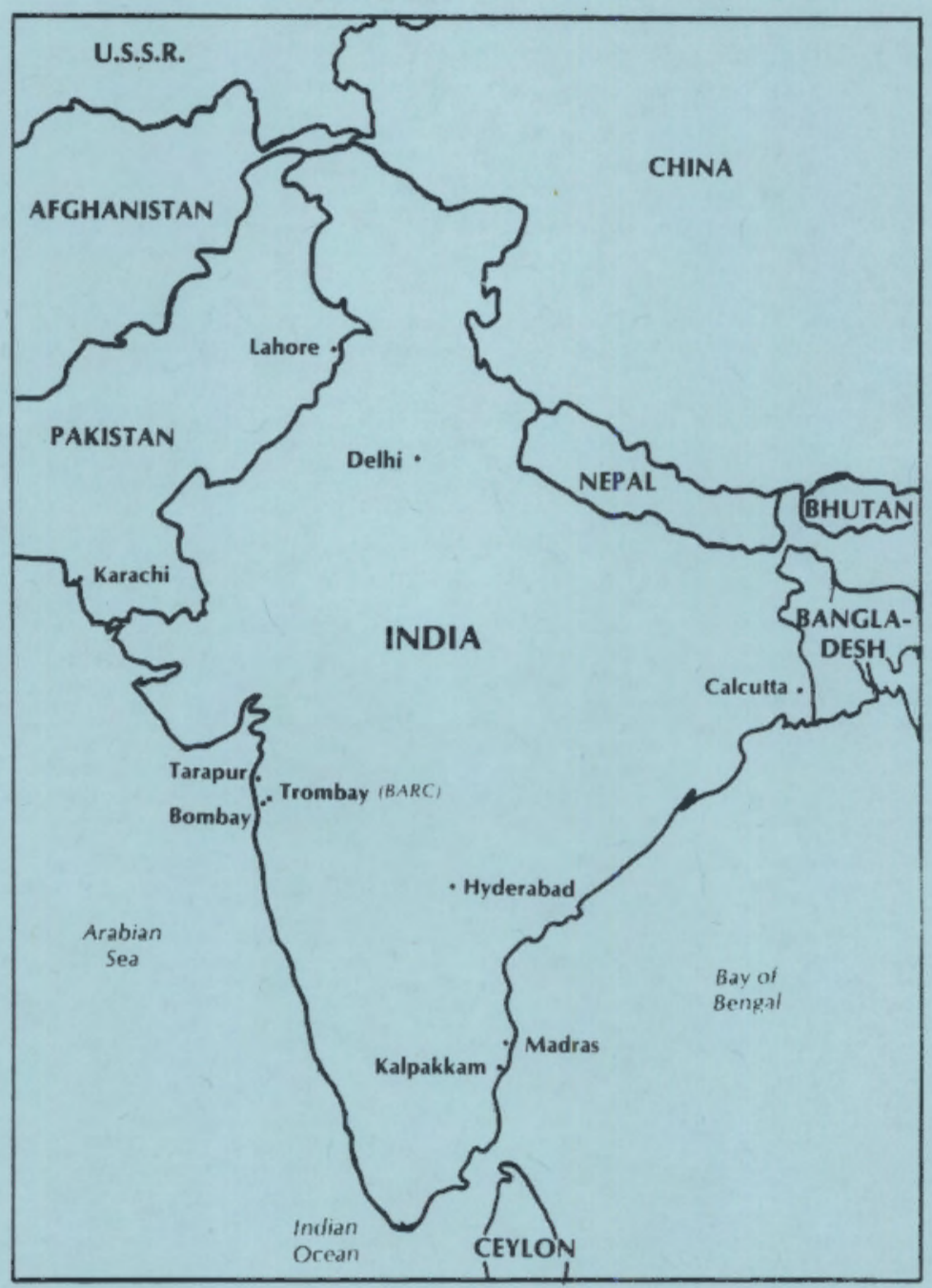




\section{INDIA}

ENERGY

$\begin{array}{lcc}\text { Population } & 1981 & 684 \text { million } \\ \text { Energy Demand } & 1979 & 94 \text { Mtoe } \\ \text { Oil Demand } & 1980 & 28 \text { Mtoe } \\ & & (65 \% \text { imported) } \\ \text { Electric Power Plant Capacity } & 1981-82 & 35 \text { GWe-- } \\ & 1985 & 2.2 \% \text { nuclear } \\ & & 50 \text { GWe } \\ & 1982 & 107 \text { TWh-- } \\ & & 2.7 \% \text { nuclear } \\ \text { Electric Power Production } & & 50 \% \text { coal } \\ & & 42 \% \text { hydro } \\ & & 6 \% \text { oil }\end{array}$

NUCLEAR POWER

National Policy. Heavy dependence on nuclear power to augment the nation's electric power generating capacity. A three-phase program--first phase, reactors fueled with natural uranium; second phase, FBRs fueled with $\mathrm{Pu}$ produced by first-phase reactors; third phase, selfsustaining thorium-uranium cycle reactors.

$\begin{array}{lll}\text { Nuclear Power Plant Capacity } & 1983 & 1.0 \mathrm{GWe} \\ & 1985 & 1.2 \mathrm{GWe} \\ & 1990 & 1.9 \mathrm{GWe} \\ & 2000 & 4.4 \mathrm{GWe}\end{array}$

Reactor Mix

1982 BWR: 2 (1969)

HWR: $2(1973-81)$;

4 (1984-89);

2 (1992)

Reactor Development

1983

40 MWt FBR test unit

1990500 MWe commercial FBR 
INDUSTRIAL FUEL CYCLE

Policy. Achieve self-sufficiency in CANDU-type fuel

cycle-uranium milling, conversion to $\mathrm{UO}_{2}$, fuel fabrication, reprocessing (in small plants adjacent to power stations); if enriched UF 6 supply for India's BWRs is cut of $f$, they may fuel with $\mathrm{UO}_{2}-\mathrm{PuO}_{2}$.

Waste Management Strategy. Vitrification of HLW, interim storage for at least 20 years and disposal in a granite or gneiss formation.

Cumulative Spent Fuel

Arisings (LWR and HWR)
1980

1985

1990

2000

Cumulative Waste Arisings

Primary solid wastes

LLW concentrates

ILW

HLW

\begin{tabular}{|c|c|}
\hline 1982 & 2000 \\
\hline $1,700 \mathrm{~m}_{3}^{3}$ & $107,000 \mathrm{~m}_{3}^{3}$ \\
\hline $\begin{array}{r}2,500 \mathrm{~m}_{3} \\
650 \mathrm{~m}_{3} \\
350 \mathrm{~m}^{3}\end{array}$ & $\begin{array}{c}77,000 \mathrm{~m}_{3}^{3} \\
20,000 \mathrm{~m}^{3} \\
8,000 \mathrm{~m}^{3}\end{array}$ \\
\hline
\end{tabular}

Industrial-Scale Activities

1. Heavy-water production ( $t / a)$ : 1981--300; additional capacity is planned, sufficient to yield a $13,000 \mathrm{t}$ inventory by the year 2000 .

2. Uranium mining and milling $(t / a): 200$.

3. $\mathrm{UO}_{2}$ fuel fabrication $(\mathrm{t} / \mathrm{a}): 1981--100 ; 1984--210$.

4. Fuel reprocessing: Trombay pilot plant, $30 \mathrm{t} / \mathrm{a}$ (1962--); Tarapur plant, 100 t/a (1982--); Kalpakkam plant, $100 \mathrm{t} / \mathrm{a}$.

5. HLW vitrification: Tarapur (1982--). 


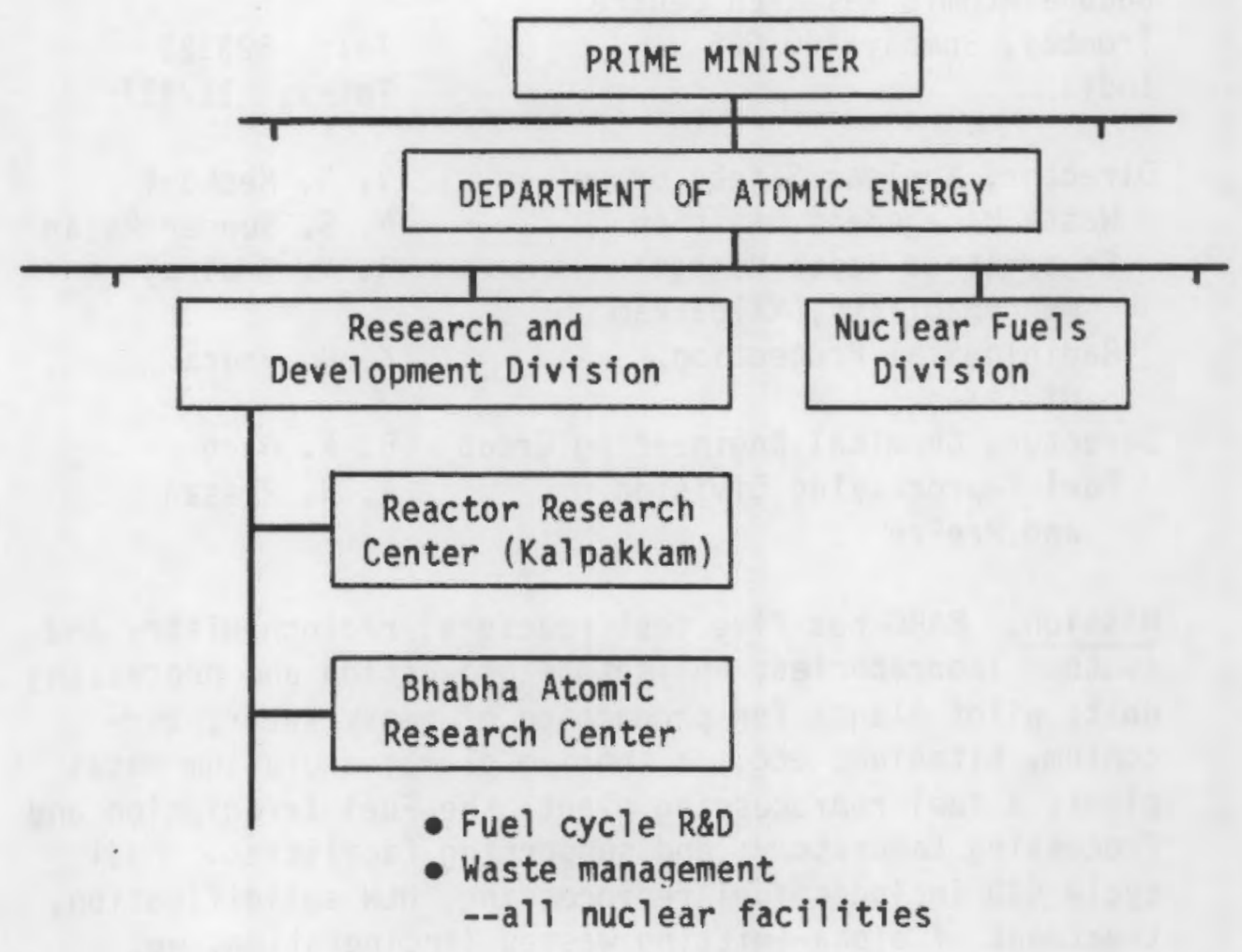

AEC (ATOMIC ENERGY COMMISSION)

Government of India

Department of Atomic Energy

Atomic Energy Comnission

Chhatrapati Shivaji Marharaj Marg

Bombay 400039

India

*Tel: $\quad 242823$

Chairman

Dr. Raja Ramanna

AERB (ATOMIC ENERGY REGULATION BOARD)

Mission. Regulation and Licensing of Nuclear Facilities.

Chairman

* No direct dialing to India.
A. X. De

(Indian Institute of Technology) 
India

BARC

Bhabha Atomic Research Centre

Trombay, Bombay 400085

India

Te1: 523321

Telex: 112817

Director, Nuclear Safety Group

V. N. Meckoni

Waste Management Division

N. S. Sunder Rajan

Centralized Waste Manage-

R. V. Amalraj

ment Facility, Kalpakkam

Radiological Protection

K. G. Vohra

Division

Director, Chemical Engineering Group

B. K. Garg

Fuel Reprocessing Division

A. N. Prasad

and PreFre

Mission. BARC has five test reactors; radiochemistry and isotope laboratories; an isotope production and processing unit; pilot plants for production of heavy water, zirconium, titanium, etc.; a thorium plant; a uranium metal plant; a fuel reprocessing plant; the Fuel Irradiation and Processing Laboratory; and supporting facilities. Fuel cycle R\&D includes fuel reprocessing, HLW solidification, treatment of alpha-emitting wastes (incineration, wet oxidation, decontamination, and immobilization of cladding hulls), D\&D, and waste isolation in geologic formations.

Facilities

1. Trombay Fuel Reprocessing Plant

Mission. Reprocess natural uranium metal fuels. Design Basis. Chemical declad, PUREX flowsheet; contact maintenance; capacity, 0.1-0.15 t HM/day. History. On-line, 1965-1974; modified and being readied to operate again.

2. HLW Vitrification Plant

History. Construction start, 1981. 
KOLAR WASTE DISPOSAL RESEARCH STATION

Location. In the Kolar gold mine area at the Karnataka State.

Mission. Assess the suitability of peninsular gneisses for location of a repository (in situ studies).

Description. Tunnel extended from abandoned section of one of the Kolar gold mines into a neighboring gneissic formation.

History. Startup, "soon" (after October 1979).

\section{MADRAS ATOMIC POWER PROJECT}

Madras Atomic Power Project

Kalpakkam

India

Mission. Nuclear power production, FBR development, fuel reprocessing and waste treatment, plutonium fuel fabrication for FBRs.

R\&D. FBR technology, reprocessing of FBR fuels.

\section{Facilities}

1. Kalpakkam Fuel Reprocessing Plant

Mission. Reprocess spent fuel from the Kalpakkam reactors and from the 15-MW FBTR due for commissioning in mid-1981. Design Basis. Purex process, with a separate line for FBTR mixed-carbide fuels; capacity, $0.5 \mathrm{tHM} /$ day for PHWR fuels.

2. Kalpakkan Fuel Reprocessing Laboratory

Mission. Develop and test equipment and unit operations for FBR fuel reprocessing.

3. Kalpakkam HLW Vitrification Plant

Milestone. Construction start, 1983. 
India

\section{NUCLEAR FUEL COMPLEX}

Nuclear Fuel Complex

Department of Atomic Energy

Cherlapalli, near Moula Ali

Hyderabad, 500040

India

Tel: $78371,71801-4$

Telex: 015304

Chief Executive

Dr. Rao

Mission. Integrated fuel and nuclear component manufacturing complex to supply Indian nuclear power plants.

\section{TARAPUR ATDMIC POWER STATION}

Tarapur Atomic Power Station

Tarapur, Maharashtra

India

Mission. Provide electric power for India, reprocess spent fuel from Tarapur reactors and immobilize the associated wastes.

Facilities

1. Tarapur Fuel Reprocessing Plant (Prefre)

Mission. Reprocess natural and low-enriched $\mathrm{UO}_{2}$ fuels (zircaloy clad).

Design Basis. Chop-leach head-end; PUREX flowsheet;

contact maintenance; capacity, 0.5 tHM/day.

History. Construction completed, 1975; hot operation, $12 / 82$.

2. WIP (Waste Immobilization Plant)

Mission. Vitrify Tarapur HLW.

Design Basis. Two-step calcination and melting in drainable pot; capacity, $25 \mathrm{l} / \mathrm{hr}$ HLLW, $125 \mathrm{~kg}$ glass/ canister, 1 canister/day; product, borosilicate glass blocks.

Milestone. Construction completed 1981; Startup, 1982. 


\section{TARAPUR ATOMIC POWER STATION (contd)}

3. Solid Storage Surveillance Facility

Mission. Provide air-cooled storage for WIP products.

Design Basis. Stack-induced natural-draft air cooling; capacity for 20 years' storage of Tarapur and Trombay WIPS.

4. ILW Bituminization Plant

Mission. Immobilize LLW.

Design Basis. Capacity--120 l/hr LLW; wiped film evaporation.

5. Polymerization Facility

Mission. Immobilize low heat generating waste (LHGW).

Design Basis. LHGW is mixed with polyesterstyrene and vermiculite in conditioning tanks emplaced in a concrete trench. 



\section{ITALY}




\begin{tabular}{ll} 
*Asterisks denote & \multicolumn{2}{c}{ ITALIAN HOLIDAYS } \\
January 1 & New Year's Day \\
*March/April & Easter Monday \\
April 25 & Liberation Day \\
May 1 & Labor Day \\
August 15 & Assumption Day \\
November 1 & All Saints' Day \\
December 8 & Immaculate Conception \\
December 25-26 & Christmas
\end{tabular}

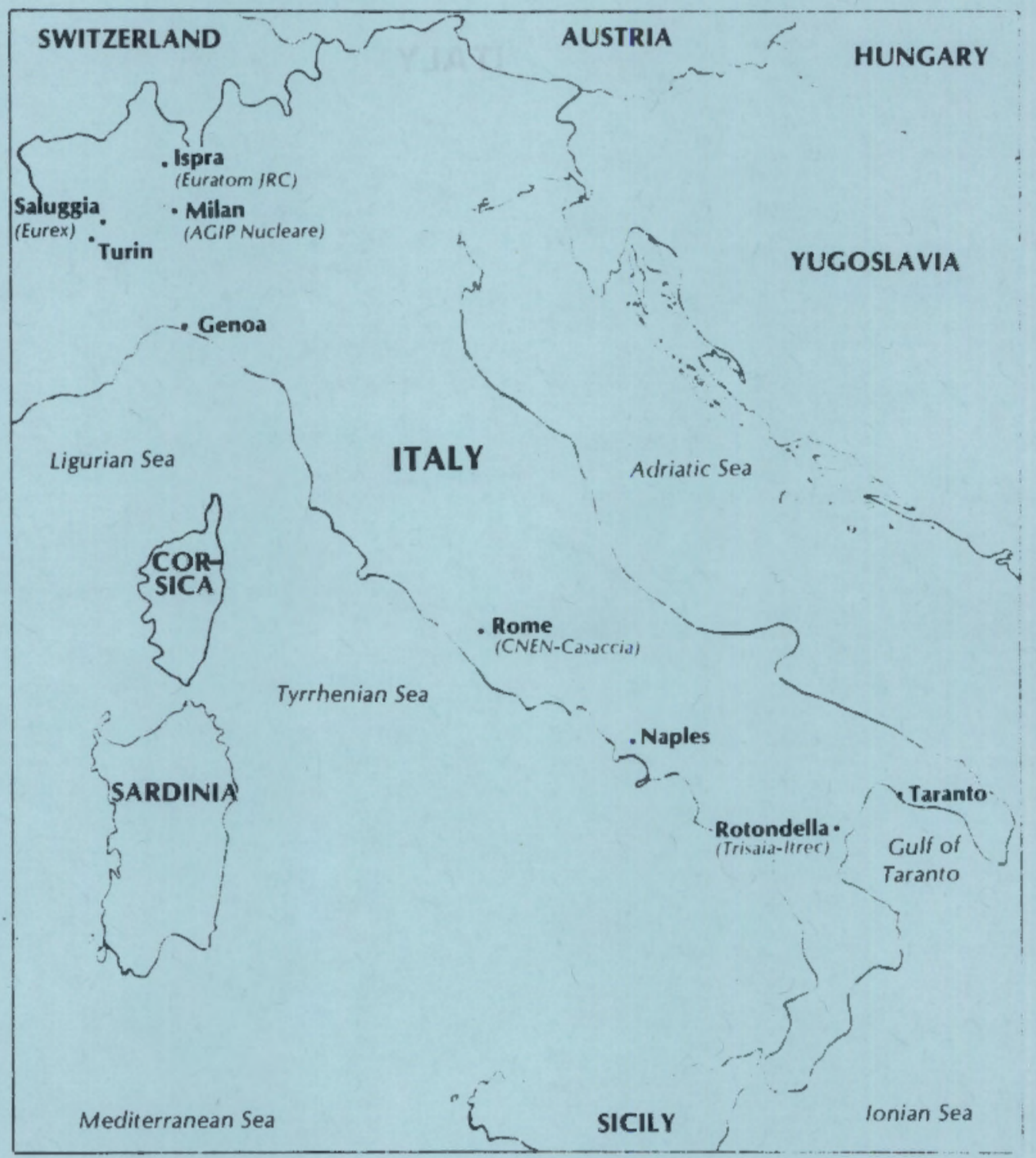


ITALY

ENERGY

\begin{tabular}{|c|c|c|}
\hline Population & 1981 & 57 million \\
\hline Energy Demand & 1980 & $\begin{array}{l}142.1 \text { Mtoe } \\
\text { (80\% imported) }\end{array}$ \\
\hline $0 i 1$ Demand & 1980 & $\begin{array}{l}95 \text { Mtoe } \\
\text { (97\% imported) }\end{array}$ \\
\hline Electric Power Plant Capacity & 1981 & $\begin{array}{l}47.4 \text { GWe-- } \\
2.7 \% \text { nuclear } \\
33.5 \% \text { hydro } \\
38.4 \% \text { oil \& gas } \\
25.4 \% \text { solid fuels }\end{array}$ \\
\hline Electric Power Production & 1982 & $\begin{array}{l}185.7 \text { Thh-- } \\
1.2 \% \text { nuclear } \\
61.3 \% \text { oil \& gas } \\
27.0 \% \text { hydro/geo. } \\
10.5 \% \text { solid fuels } \\
174 \text { TWh-- } \\
3.8 \% \text { nuclear }\end{array}$ \\
\hline
\end{tabular}

NUCLEAR POWER

National Policy. National government supports nuclear power growth, but is hampered by financial and siting problems.

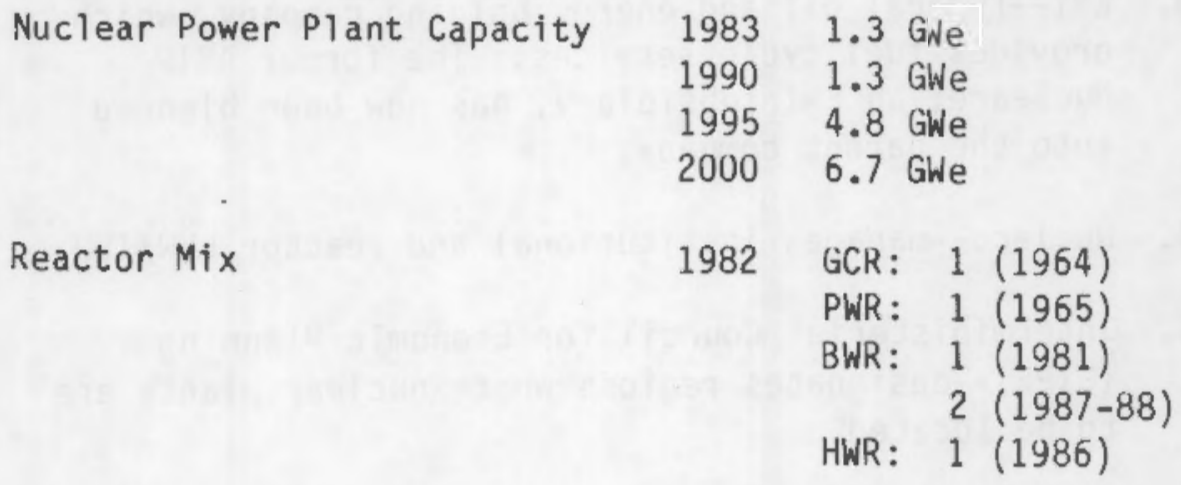

Reactor Development HWR, FBR; membership in Super Phenix consortium 
INDUSTRIAL FUEL CYCLE

Policy. Reprocess spent fuel and recycle Pu to breeders; develop indigenous fuel cycle and waste treatment capability.

Waste Management Strategy. HLW--vitrify and store in engineered surface facility for 50-60 years; emplace canisters in geologic repository (clay).

Cumulative Spent Fuel Arisings (LWR)

$$
\begin{array}{rr}
1980 & 160 \mathrm{tU} \\
1985 & 330 \mathrm{tU} \\
1990 & 520 \mathrm{tU} \\
2000 & 2,000 \mathrm{tU}
\end{array}
$$

INTERNATIONAL RELATIONSHIPS

1. Membership in European Communities, IAEA, OECD/NEA.

2. Participation in Eurodif, Coredif, Super Phenix projects.

3. A CEC Joint Research Centre laboratory is located in northern Italy at Ispra.

\section{ORGANIZATION}

1. Ministry of Industry--overall nuclear responsibility.

2. ENEA--safety and regulatory; nuclear R\&D (principally at the Casaccia, Saluggia and Trisaia Centres).

3. ENI--federal oil and energy holding company, which provides fuel cycle services. The former AGIP Nucleare, an ENI subsidiary, has now been blended into the parent company.

4. Nucleco--manages institutional and reactor LLW/ILW.

5. Interministerial Council for Economic Planning (CIPE)--designates regions where nuclear plants are to be located.

6. ENEL--state-owned power utility. 
ENEA (NATIONAL COMMISSION FOR RESEARCH AND DEVELOPMENT

OF NUCLEAR ENERGY AND ALTERNATIVE FORMS OF ENERGY)

ENEA Regina Margherita 125

I-00198 Rome

Italy

Tel: (39) 685281

Telex: 61183

Vice President

Prof. Carlo Salvetti

Director General

Fabio Pistella

Research Laboratory

Fuel Cycle Department

Guiseppe Grossi

Ing. Venditt $i$

Mission. Supervise pure and applied nuclear research, maintain technical control over nuclear power plants, cooperate in international program.

Sponsor. Federal government.

\section{ENEA: CASACCIA CENTER}

ENEA-Casaccia Center

S. Maria di Galeria

S.P. Anguilliarese km $1+300$

I-00060 Rome

Italy

For Correspondence:

Casella Postale 2400

I-00100 Rome

Italy

Director, Fuel Cycle

Waste Management

HLW Vitrification Project

Reprocessing and Waste
Tel: (39) 6 6948-1

Telex: 613296

Mission. Applied research--nuclear energy and alternative sources of energy.

Sponsor. ENEA.

Waste Management R\&D. MOX fuel reprocessing, HLW solidification, actinide transmutation, treatment of $\mathrm{LLW}$ and characterization of waste forms, waste isolation in clay formations (site characterization and thermal properties). 


\section{ENEA: SALUGGIA CENTER}

ENEA-Impianto Eurex

I-13040 Saluggia (Vercelli)

Tel: (39) 16148415

Italy

Telex: 380058 EURI

(Located about $35 \mathrm{~km}$ from Torino and $120 \mathrm{~km}$ from Milano.)

Director, Eurex Pilot Plant

Dr. Franco Pozzi

Deputy Director, Eurex

Arnoldo Hall

Sponsor. ENEA.

Mission. Applied nuclear research (EUREX fuel reprocessing pilot plant is located here).

\section{Facilities}

1. EUREX (fuel reprocessing pilot plant-radioactive)

Mission. Reprocess MTR and low-enriched uranium (including $\mathrm{UO}_{2}$ ) fuels.

Design Basis. PUREX process; chop-leach head-end has been added; capacity, $30 \mathrm{~kg} \mathrm{U-Al/day} \mathrm{of} \mathrm{MTR} \mathrm{fuels,}$ $50-100 \mathrm{~kg} /$ day of LWR oxides.

History. Built and operated under a CNEN-Euratom convention 1964-1983. Startup, 1970. CANDU fuels from Canada processed in 1983. A unit is to be added for MOX fuel reprocessing.

2. IVEX (HLW vitrification plant-radioactive)

Mission. Immobilize EUREX HLW.

3. IFEC (fuel element fabrication)

Mission. Fabricate ceramic uranium fuel for Cirene research reactor. 


\section{ENEA: TRISAIA CENTER}

ENEA-CRE Trisaia

S.S. 106 Jonica, Km. 419+500

I-75025 Policoro (Matera)

Italy
Tel: (39) 835972241

Telex: 760085 ENEATR I

(Located near Rotondella, about $5 \mathrm{~km}$ from the coast of the Ionian Seas in the Gulf of Taranto.)

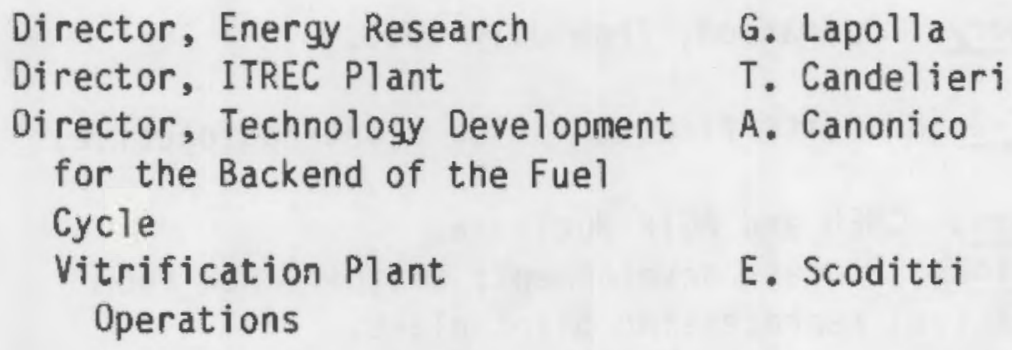

Mission. Applied nuclear research (ITREC fuel reprocessing pilot plant is located here).

Sponsor. ENEA.

Waste Management R\&D. Fuel reprocessing, centrifugal contactor development, cladding hulls compaction, HLW vitrification, D\&D, and waste isolation (clay repositories).

\section{Facilities}

1. ITREC (fuel reprocessing pilot plant-radioactive)

Mission. FBR fuel reprocessing R\&D; reprocess thorium and MOX (FBR) fuels. Design Basis. Chop-leach head-end; maintenance by remote removal of modules; capacity, $15 \mathrm{~kg} \mathrm{HM} /$ day $\left(\mathrm{ThO}_{2}\right.$ and $\mathrm{UO}_{2}$ ). History. Startup, 1975; $10 \mathrm{~m}^{3}$ HLLW produced. 


\section{ENEA: TRISAIA CENTER (contd)}

2. IVET-1 (vitrification pilot plant-nonradioactive)

Sponsors. CNEN and AGIP Nucleare. Mission. Develop HLW vitrification process. Design Basis. IVET-1 pot vitrification (two-stage, rising level process); capacity, $20 \mathrm{l} / \mathrm{hr}$ feed; product, borosilicate glass cylinders, $0.25 \mathrm{~m}$ dia $x$ $1 \mathrm{~m}$. History. Operation, from July 1980.

3. IVET-2 (HLW vitrification pilot plant-radioactive)

Owners. CNEN and AGIP Nucleare. Mission. Process development; solidify HLW from EUREX fuel reprocessing pilot plant. Design Basis. Pot vitrification (two-stage, rising level process); capacity, $15 \mathrm{l} / \mathrm{hr}$ feed (2 canisters/ wk) or $10 \mathrm{~m}^{3} \mathrm{HLLW} / \mathrm{a}$; product, borosilicate glass cylinders, $0.25 \mathrm{~m}$ dia $\times 1 \mathrm{~m}$. History. Startup, late 1980s.

\section{ENEL (NATIONAL ELECTRIC ENERGY AGENCY)}

Ente Nazionale per L'Energia Elettrica

Casella Postale 386

Via Giovan Battista Martini 3

I-00198 Rome

Italy

Vice President

Director General
Tel: (39) 685091

Telex: 610518

L. Magno

M. Morett $i$

Mission. Responsible for electric power produced by all sources.

Sponsor. Federal government. 
ENI

Ente Nazionale Indrocarburi SPA

San Donato Milanese

I-20093 Milan

Italy

Tel: (39) 2 5353-1

Telex: 34192

President

Mr. Franco Reviglio

Mission. 0il and energy holding company, owned by the federal government.

NUCLECO

Nucleco

Via Europa-Cologno Monzese 46

I-20093 Milan

Italy

Mission. Treat and dispose of low- and intermediate-level wastes from hospitals, laboratories, industrial establishments, and nuclear powerplants. Eventual plans include decommissioning work on nuclear installations.

Sponsor. Italian government (CNEN--40\%; AGIP--60\%).

\section{SNIA TECHINT}

Snia Techint

Tecnologie Energetiche Avanzate SPA

Via A. Bargoni 34

I-00153 Rome

Tel: (39) 65894041

Italy

Telex: 614354 SNITEC

General Manager

Marino Fiorelli

Mission. Provide architect-engineering services for reprocessing, fuel handling and HLW conditioning

facilities. 

JAPAN 


\begin{tabular}{ll} 
& \multicolumn{2}{c}{ JAPANESE HOLIDAYS } \\
*Asterisks denote movable holidays & \\
January 1 & New Year's Day \\
January 15 & Adult's Day \\
February 11 & National Foundation Day \\
*March & Vernal Equinox \\
April 29 & Emperor's Brithday \\
May 3 & Constitution Day \\
May 5 & Children's Day \\
September 15 & Respect for the Aged Day \\
*September & Autumnal Equinox \\
October 10 & Physical Culture Day \\
November 3 3 & Culture Day \\
November 23 & Labor Thanksgiving Day
\end{tabular}

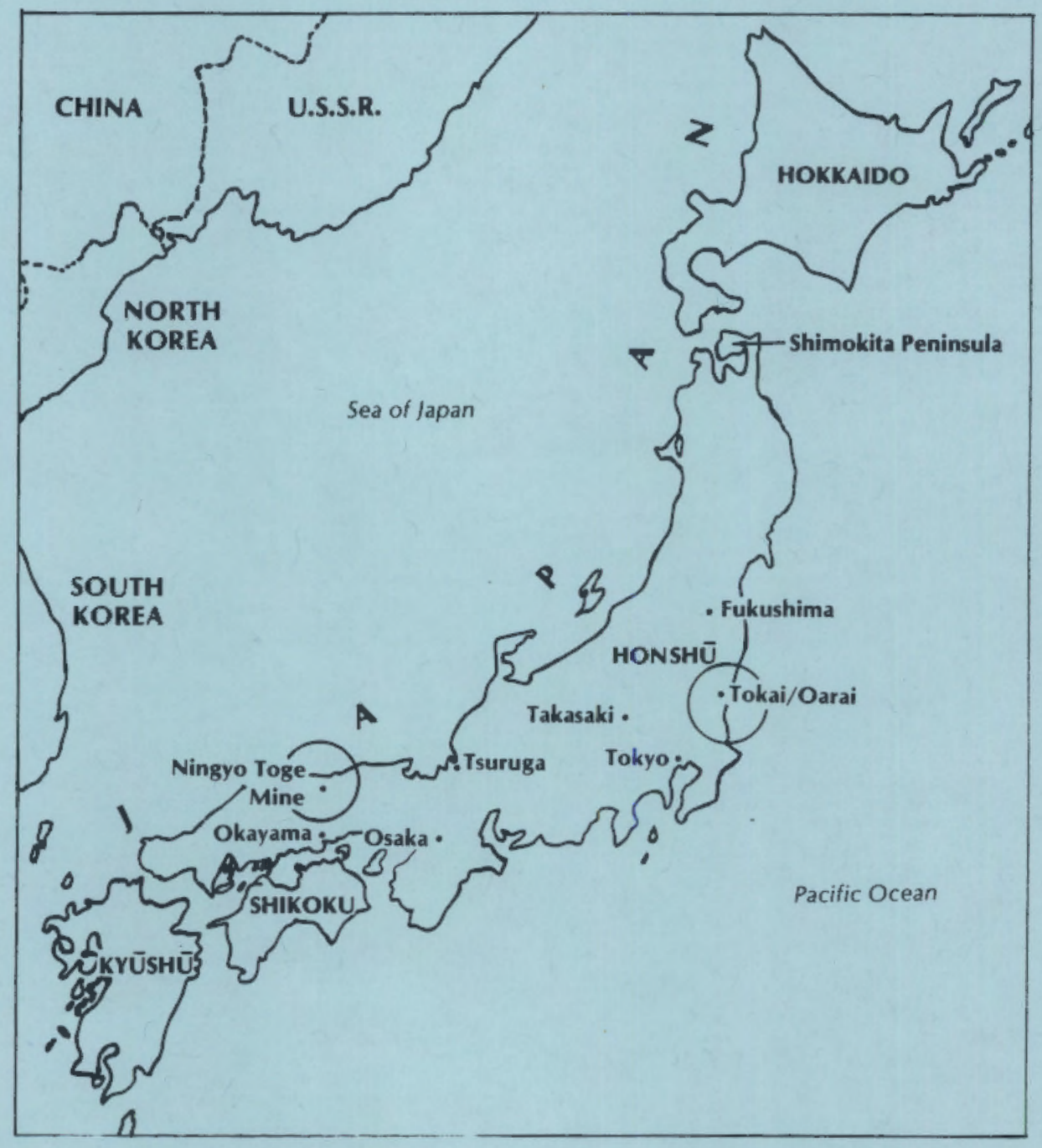


JAPAN

\section{ENERGY}

\begin{tabular}{|c|c|c|}
\hline Population & 1982 & 118.4 million \\
\hline Energy Demand & $\begin{array}{l}1980 \\
1990\end{array}$ & $\begin{array}{l}370 \text { Mtoe } \\
\text { ( } 85 \% \text { imported) } \\
622 \text { Mtoe } \\
\text { ( } 78 \% \text { imported) }\end{array}$ \\
\hline 0i] Demand & 1980 & $\begin{array}{l}255.4 \text { Mtoe } \\
\text { (99\% imported) }\end{array}$ \\
\hline Electric Power Plant Capacity & $\begin{array}{l}1982 \\
1985\end{array}$ & $\begin{array}{l}137 \text { GWe-- } \\
13 \% \text { nuclear } \\
170 \text { GWe }\end{array}$ \\
\hline Electric Power Production & 1982 & $\begin{array}{l}577.5 \text { TWh-- } \\
14.3 \% \text { nuclear } \\
61.6 \% \text { oil \& gas } \\
16.2 \% \text { hydro/geo. } \\
7.9 \% \text { solid fuels } \\
511 \text { TWh-- } \\
20.3 \% \text { nuclear }\end{array}$ \\
\hline
\end{tabular}

NUCLEAR POWER

National Policy. Strong nuclear power program, to lessen dependence on foreign energy sources--install LWRs for near-term needs; develop an advanced HWR; aim for commercial FBR operation by the year 2010. Supply domestic needs and build export business.

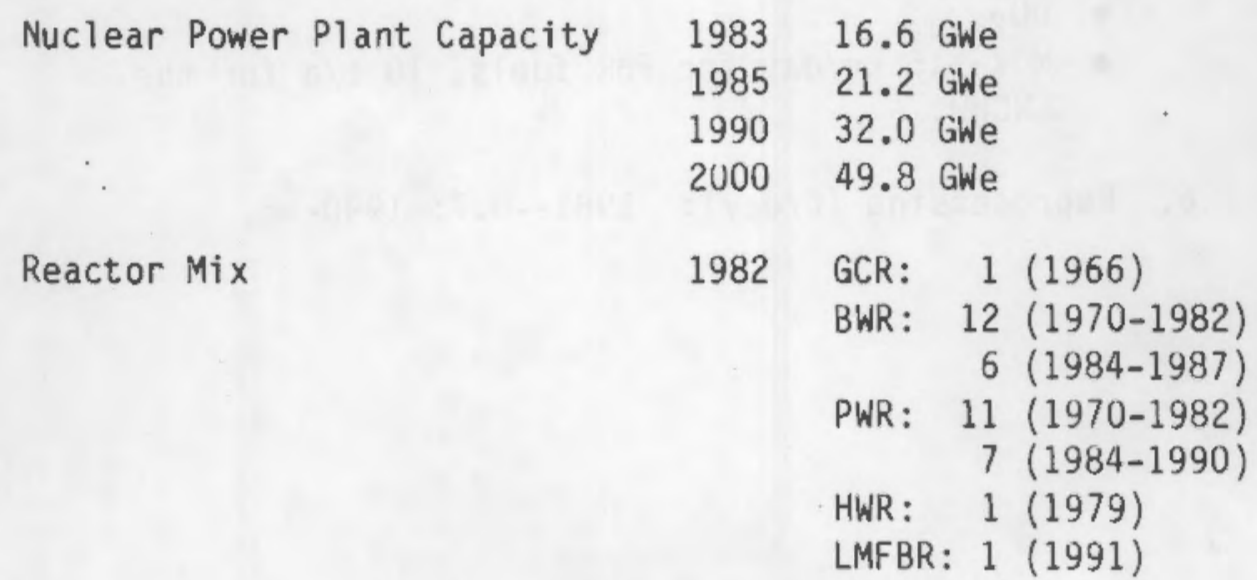




\section{INDUSTRIAL FUEL CYCLE}

Policy. Obtain ownership of foreign uranium resources; develop complete fuel cycle capability (enrichment, reprocessing and waste treatment, buying foreign reprocessing services as long as necessary); recycle Pu to FBRs, HWRs, and LWRs.

Waste Management Strategy. HLW--vitrify and store in engineered surface facility until repository is ready. LLW--sea dump is preferred, but land disposal is being developed in case sea dump option is not available. Preferred geology for HLW repository has not been selected.

Cumulative Spent Fuel Arisings (LWR)

$$
\begin{array}{rr}
1980 & 1,450 \mathrm{tU} \\
1985 & 4,100 \mathrm{tU} \\
1990 & 8,200 \mathrm{tU} \\
2000 & 20,000 \mathrm{tU}
\end{array}
$$

Industrial-Scale Activities

1. Power production (GWe): 1981--16.4; 1990--51.

2. Uranium mining and milling (tU/a): 30 (domestic).

3. Conversion (tUF $6 / a)$ : 200.

4. Uranium enrichment (tSWU/a): 1981--75; 1985--1000; $2000--3000$.

5. Fuel fabrication

- $\mathrm{UO}_{2}$

- MOX--15 kg/day for FBR fuels, $10 \mathrm{t} / \mathrm{a}$ for the LWCHW.

6. Reprocessing (t/day): 1981--0.7; 1990--6. 
Major Milestones

- HLW solidification pilot plant (PNC)

- Commercial reprocessing plant for LWR fuels

- FBR fuel reprocessing pilot plant

- HLW solidification commercial plant

- Return of HLW from COGEMA and BNFL

- Experimental sea-dumping of LLW

- Selection of candidate geological or $\sim 1985$ seabed repository formations

- Selection of repository test site for in situ test with a simulated waste package

- Selection of demonstration site for in situ test with actual waste package

- Startup of disposal site

- MONJU LMFBR

INTERNATIONAL RELATIONSHIPS

1. Member of IAEA and OECD/NEA.

2. LMFBR exchange agreement with DOE.

3. Licensing agreements with GE and Westinghouse.

ORGANIZATION

Government funds nuclear $R \& D$ and is responsible for $H L W$ disposal. Industry handles the commercial fuel cycle and LLW disposal, and pays for HLW disposal. See Figures $J A-1, J A-2, J A-3$ for organizational relationships and responsibilities. 
Industrial Sponsors

(Utilities, Manufacturers, etc.)

\section{CRIEPI}

- Properties of LLW

packages

apan Nuclear Fuel

Services Co., JNFS

- Comnercial reprocessing

and waste treatment

- HLW storage

Radioactive Waste

Management Center

- LLW disposal-R\&D

and operations

- Environmental monitoring \& safety

Kobe Steel, Ltd.

- Waste management

equipment development

Mitsubishi Metal Corp.

- Waste treatment equipment and systems

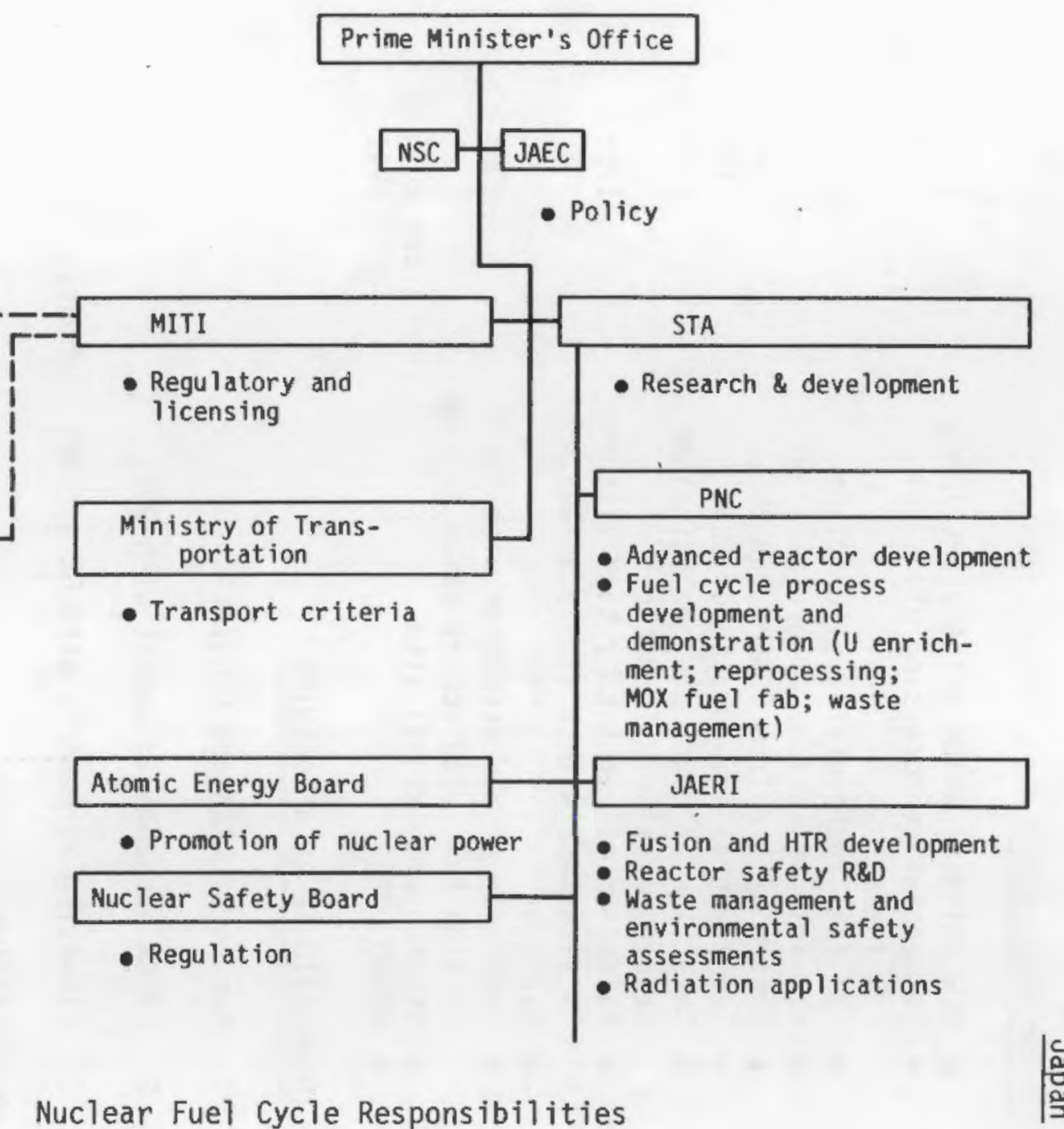

FIGURE JA-1. Nuclear Fuel Cycle Responsibilities 


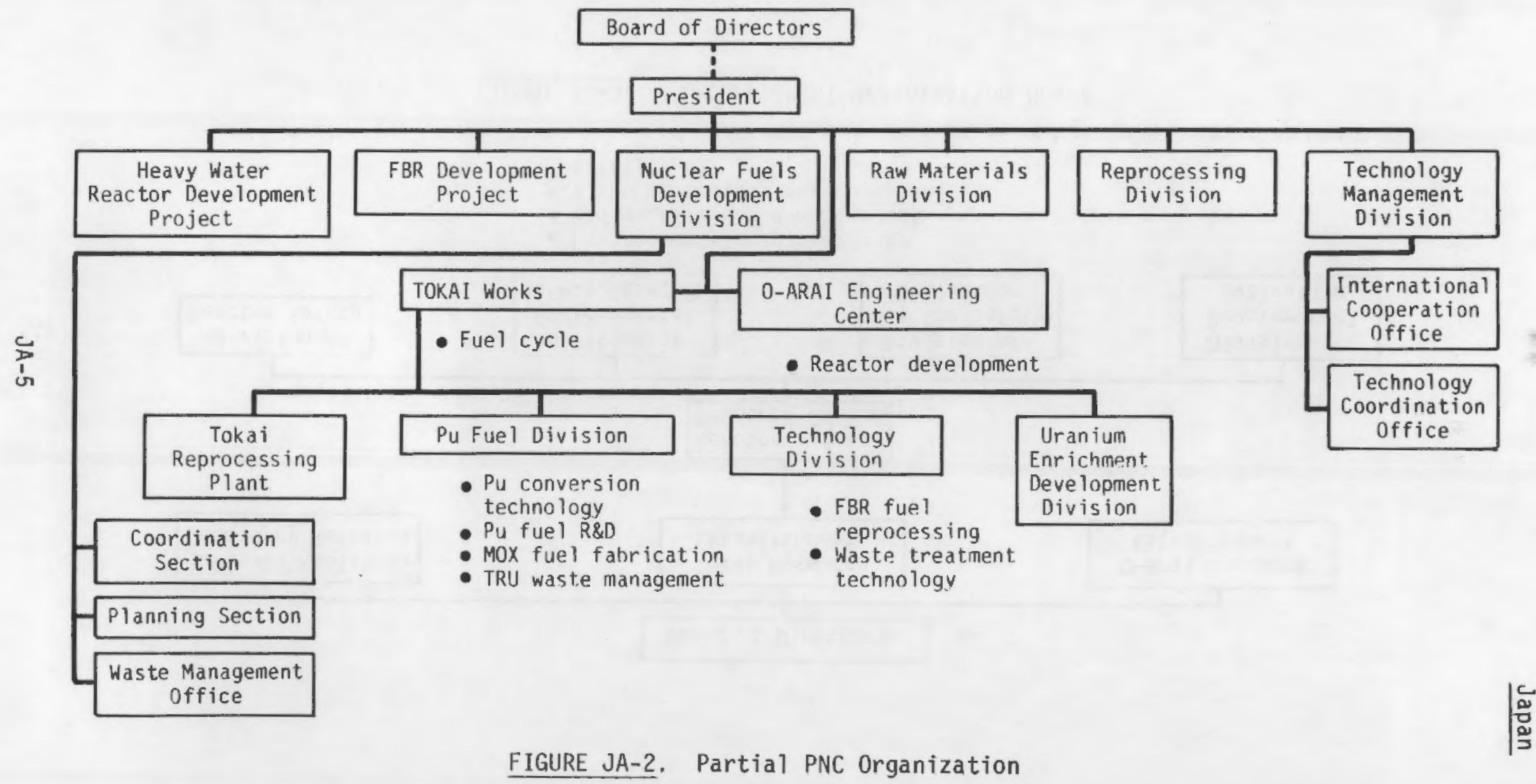




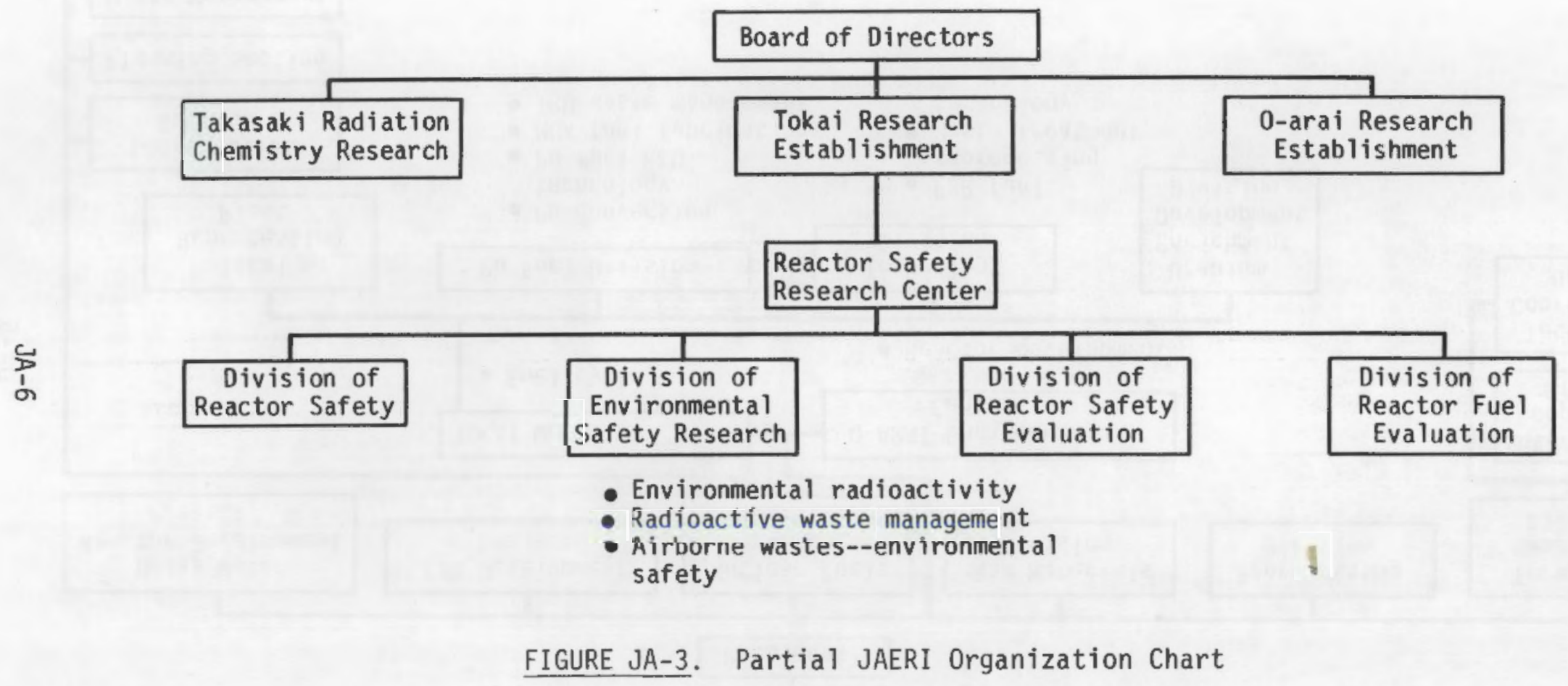




\section{AEC (ATOMIC ENERGY COMMISSION)}

Atomic Energy Commission

2-2-1 Kasumigaseki

Chiyoda-ku, Tokyo

Japan

Tel: (81) $3581-2585$

Chairman

T. Yasuta

Mission. Formulate national policy on nuclear energy

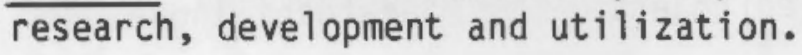

\section{CRIEPI (CENTRAL RESEARCH INSTITUTE \\ OF ELECTRIC POWER INDUSTRY)}

Civil Engineering Laboratory

1646, Abiko, Abiko-Shi,

Chiba Pref. 270-11

Japan

Te1: (81) $47182-1181$

Energy and Environmental Laboratory

11-1, Iwato-kita, 2-chome,

Komae-shi, Tokyo 201

Tel: (81) 3 480-2111

Japan

Telex: 2423098 CRIEPI J

GIRIO

Government Industrial Research

Institute, Osaka

1-8-31 Midorigaoka, Ikeda-shi

Osaka 563, Japan

Tel: (81) 727 51-8351

Director, 4th Department

Dr. Ryozo Hayami

Nuclear Waste Program

Dr. Ryohei Terai

R\&D. Alternatives for HLW solidification; waste form characterization. 
Japan

\section{HITACHI}

Hitachi Engineering Co., Ltd.

1-1 Saiwai-cho, 3-chome

Hitachi-shi, Ibaraki-ken, 317

Japan

Tel: (81) 294 21-1111

Telex: 03645511

Nuclear Power Plant Construction

Kiyoshi Shimizu

Department

Nuclear Fuel Project

Yasuo Hirose

Nuclear Fuel Cycle Project

Sadatoshi Inoue

Fuel Cycle R\&D. Develop technology to reprocess spent LWR fuel; fixation, storage, and disposal of HLW; spent fuel storage; $\mathrm{Pu}$ fuel production; and decommissioning.

\section{JAER I}

Japan Atomic Energy Research Institute

2-2-2, Uchisaiwaicho

Chiyoda-ku, Tokyo 100

Japan

Tel: (81) 35036111

Telex: $\mathbf{3 2 4 5 9 6}$

Location. JAERI headquarters and Radioisotope Center are in Tokyo. The Tokai and 0-arai Research Establishments share government reservations at Tokai-mura and 0-araimachi with PNC. Tokai and 0-arai are 120 and $100 \mathrm{~km}$, respectively, northeast of Tokyo, near the ocean. These sites can be reached by train from Tokyo to the city of Mito, then by taxi.

\section{JAERI： 0-ARAI}

0-Arai Research Establishment

0-Arai-machi, Hi gashi-Ibaraki-gun

Ibaraki-ken 311-13

Japan

Tel: (81) $29267-4111$ 


\section{JAERI: TOKAI}

Tokai Research Establishment

Tokai-mura, Naka-gun

Ibaraki-ken 319-11

Japan

Director

Director, Division of Environmental Safety Research (DERS)

Deputy Director, DERS
Tel: (81) 2928 2-5111

Telex: $3632340 \mathrm{~J}$ TOKAI J

Dr. H. Ishikawa

Dr. H. Amano

Dr. K. Araki

\section{$\underline{\text { Facilities }}$}

WASTEF (glove box and hot cell facilities)

Mission. Safety evaluations for high level waste. History. Startup, cold tests, 1981; hot tests, 1982.

\section{JAPAN NUCLEAR FUEL SERVICE COMPANY}

Japan Nuclear Fuel Service Company, Ltd.

2-2, 2-chome, Uchisaiwaicho

Chiyoda-ku, Tokyo 100

Japan

President

General Manager

Managing Director and Gen'l

Mgr., International

Affairs Dept.
Tel: (81) 35806911

Kiyoshi Goto

Shigeru Sato

Shigefumi Tamiya

\section{Facilities}

Commercial Fuel Reprocessing Plant

Mission. Reprocess Japanese fuels. Design Basis. Two lines $3 \mathrm{tHM} /$ day; $3000 \mathrm{tU}$ storage pool; HLW vitrification and storage. Cost estimate 690 billion yen.

Milestone. FRP startup, 1990; HLW vitrification and storage, 1993. 


\section{JGC CORPORATION}

Nuclear Project Division

New Ontemachi Bldg., 2-1

Ohtemachi 2-chome

Chiyoda-ku, Tokyo 100

Tel: (81) 3 279-5441

Japan

Telex: 02223096 JGCTOK J

Research and Development Division

14-1, Bessho 1-chome

Minami-ku,

Yokohama 232

Tel: (81) 45 712-1111

Japan

Telex: 03822451 JGCYOK J

Mission. Design and construction of fuel reprocessing and radwaste treatment facilities.

Waste Management R\&D. Treatment of reactor wastes (acid digestion of resins, immobilization of residues).

\section{Facilities}

Acid Digestion Pilot Plant

Mission. Provide engineering-scale test of process for treatment of reactor wastes. Milestone. Startup, 1984. 


\section{KOBE STEEL}

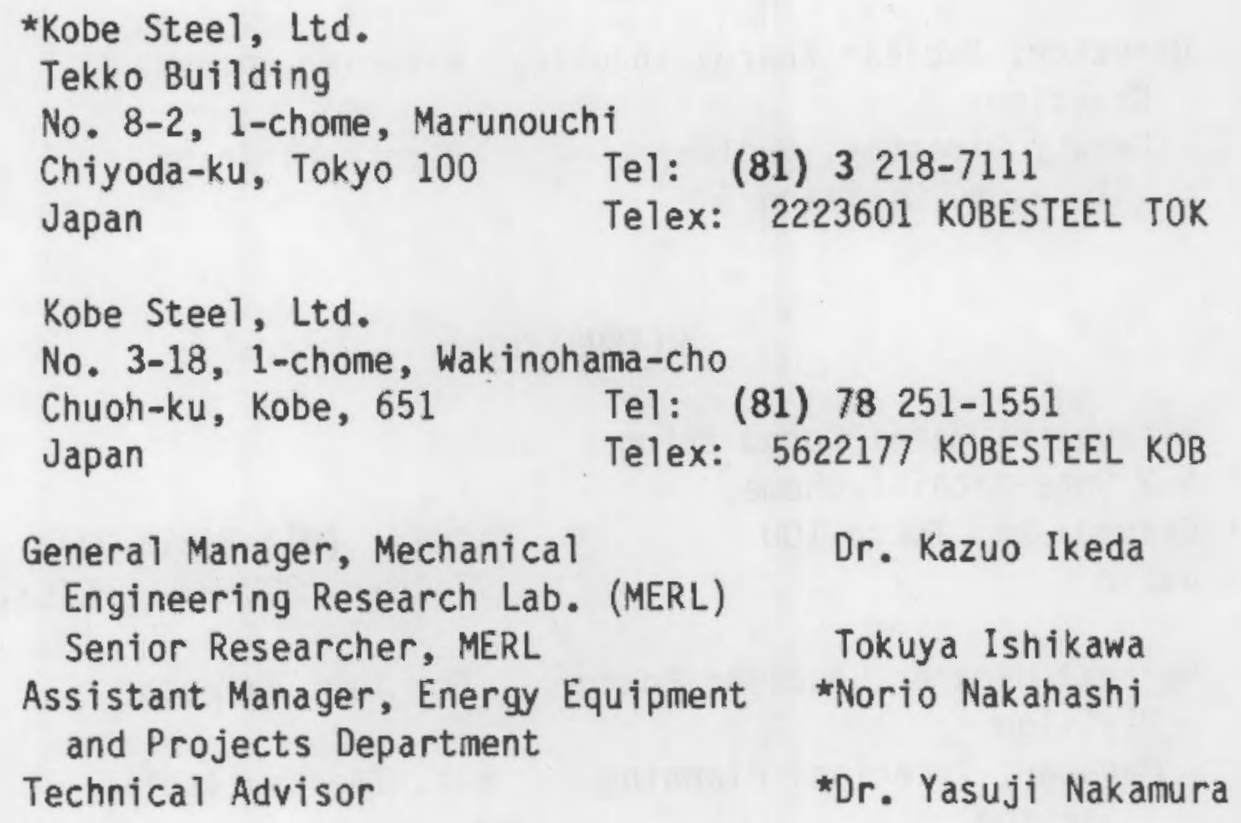

\section{ME IDENSHA}

Meidensha Electric Manufacturing Co., Ltd.

New Ohtemachi Building

2-1, 2-chome, Ohtemachi

Chiyoda-ku, Tokyo 100

Tel: (81) 3 246-7086

Japan

Telex: 02223507 Meiden J

Senior Managing Director and Director H. Hamoda of Development Division

R\&D. Remote maintenance systems for PNC fuel cycle activities.

\section{MITI (MINISTRY OF INTERNATIONAL TRADE AND INDUSTRY)}

Ministry of International Trade and Industry 3-1, Kasumi gasek $i$

Chiyoda-ku, Tokyo 100 Japan

Tel: (81) 3 501-1511 


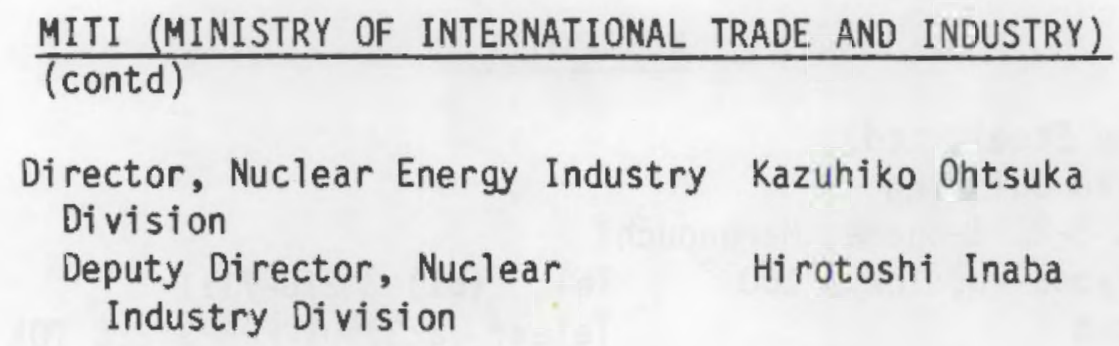

\section{MITSUBISHI}

Mitsubishi Metal Corporation

5-2 Onte-machi 1-chome,

Chiyoda-ku, Tokyo 100

Japan

Te1: (81) 3 213-2111

Japan

Telex: 2226533 MMCTOKJ

General Manager, Nuclear Energy

Dr. Yumi Akimoto

Division

Manager, Technical Planning

Section

General Manager, Technology

Department

General Manager, Nuclear

Resources Development and

Waste Management

Dr. Tamotsu Ishii

Eiji Yagi

Takaaki Kashiwagi

R\&D. Design and research on facilities for spent fuel storage and reprocessing, waste treatment and geologic disposal.

\section{NSC (NUCLEAR SAFETY COMMISSION)}

Nuclear Safety Commission

2-2-1, Kasumi gaseki

Chiyoda-ku, Tokyo 100

Japan

Tel: (81) 3 581-5271

Chairman

Tokuo Suita 
PNC

Power Reactor and Nuclear Fuel Development

Corporation

Sankaido Building

1-9-13 Akasaka

Minato-ku, Tokyo 107

Tel: (81) 35863311

Japan

Telex: J26462

President

M. Yoshida

Executive Director, FBR R\&D

K. Mochizuki

Executive Director; Director

K. Uematsu Nuclear Fuels Development

Deputy Director

T. Nishiya

Manager, Waste Management

T. Nishiya (Acting)

Director, Reprocessing

T. Koi zumi

Director, Technology Management

M. Koizumi

Manager, Internationa 1

C. Kinoshita

Cooperation

DOE Representative

D. H. Jones (HEDL)

Ext. 493

US Office:

Power Reactor and Nuclear Fuel

Development Corporation

Suite 908

2600 Virginia Avenue N.W.

Washington, DC 20037

Te1: (202) 338-3770

Telex: 892777

Manager, US Office

Kikuo Nagasawa

\section{PNC: 0-ARAI}

PNC 0-Arai Engineering Center

Oarai-machi, Higashi Ibaraki-gun

Ibaraki Pref. 311-13

Japan

Di rector

Waste Management
Te1: (81) $29267-4141$

Telex: $\mathbf{J} 26482$

K. Tanaka

S. Masuda 
PNC: TOKAI

PNC Tokai Works

Muramatsu 3371,

Tokai-mura, Naka-gun

Ibaraki-ken 319-11

Japan

Tel: (81) 2928 2-1111

Director

M. Ichikawa

Deputy Director

T. Watanabe

Director, Reprocessing Plant

G. Fukuda

Director, Processing

T. Yamanouchi

Director, Reprocessing Technology

K. Matsumoto

Development

Director, Fuel Cycle Technology

M. Yamamoto

Development

Director, Plutonium Fuel

Director, Conversion Technology

S. Watanabe

N. Tsujo

Facilities

1. Fuel Reprocessing Plant

Mission. Reprocess low-enriched $\mathrm{UO}_{2}$ and natural

uranium metal fuels.

Design Basis. Metal fuels: mechanical decladding.

Oxide fuels: chop-leach head-end. Purex flowsheet;

capacity, 0.7 tHM/day. Remote maintenance of chop-

leach equipment; contact maintenance of other

components.

History. Startup, September 1977; 170 tU in spent

fuel have been processed through December 1983.

2. Co-Conversion Facility

Mission. Demonstrate PNC microwave process for coConversion production of MOX.

Design Basis. $10 \mathrm{~kg} /$ day $\mathrm{MOX}\left(50 \% \mathrm{PuO}_{2}, 50 \% \mathrm{UO}_{2}\right)$.

Milestone. Start-up, 1984. 
PNC: TOKAI (contd)

3. Plutonium Fuel Fabrication Facility (PFFF)

Mission. Fabricate FBR and ATR fuels.

Design Basis. FBR fuels- $15 \mathrm{~kg} / \mathrm{d}\left(30 \% \mathrm{PuO}_{2}\right.$ in

enriched $\left.\mathrm{U0}_{2}\right)$; ATR fuels- $-10 \mathrm{t} / \mathrm{a}\left(2 \% \mathrm{PuO}_{2}\right.$ in $\left.\mathrm{UO}_{2}\right)$.

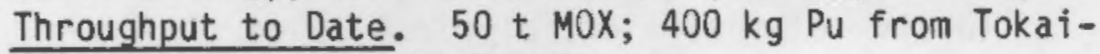
mura has gone into FUGEN ATR.

4. Fuel Fabrication Plant

Mission. Fabricate MOX fuel for FUGEN MONJU, new ATR, and LWRs.

Design Basis. $40 \mathrm{t}$ MOX/a, expandable to $140 \mathrm{t} / \mathrm{a}$.

MONJU fuel contains $30 \% \mathrm{PuO}_{2}$.

5. Vitrification Pilot Plant

Mission. Vitrify and store HLW from the Tokai-mura fuel reprocessing plant; demonstrate technology. Design Basis. Ceramic melter to produce a borosilicate glass (tentative); capacity, $0.79 \mathrm{~m}^{3} \mathrm{HLLW} /$ day or 1 canister (76 $\ell$ )/day of glass. Milestone. Startup, 1987.

6. Mockup Test Facilities

Mission. Nonradioactive, full-scale tests of vitrification and fuel reprocessing processes and equipment.

Milestone. Startup, March 1982 (vitrification); reprocessing, mid-1983.

\section{HLW Vitrification Facility}

Mission. Develop ceramic melter technology. Design Basis. Joule-heated melter-- $45 \mathrm{~kg} / \mathrm{hr}$ glass; induction-heated melter $-7.5 \mathrm{~kg} / \mathrm{hr}$ glass. 
PNC: TOKAI (contd)

8. Engineering Test Facility (reprocessing and HLW treatment)

Mission. Industrial-scale, nonradioactive studies of FBR spent fuel reprocessing and HLW solidification (pulse columns, electrolytic Pu/U partitioning, offgas treatment, solvent treatment, ceramic melters). Design Basis. Reprocessing--Purex; HLW--evaporation and denitration, vitrification (ceramic melter); melter capacity, 80 e/day glass. History. Facility startup, February 1980.

9. Chemical Processing Facility (reprocessing and HLW treatment)

Mission. Radioactive studies of spent fuel reprocessing (FBR and other advanced fuels) and HLW solidification processes. Design Basis. Five standard hot cells for breederfuel reprocessing R\&D, five cells for waste conditioning R\&D. Reprocessing--1 kg/batch; HLW solidification--30 $\mathrm{e} / \mathrm{hr} \mathrm{HLW}, 6 \mathrm{~kg} / \mathrm{hr} \mathrm{glass}$.

Milestones. Cold tests, 1981; hot tests, March 1982.

10. FBR Fuel Reprocessing Test Facility (reprocessing and HLW treatment)

Mission. Demonstrate FBR fuel reprocessing and HLW solidification. Design Basis. $120 \mathrm{~kg} \mathrm{M0X/day} \mathrm{(12} \mathrm{t/a).}$ History. Hot operation, 1981.

11. $85 \mathrm{Kr}$ Recovery Pilot Plant

Mission. Demonstrate $85 \mathrm{Kr}$ recovery from Tokai-mura reprocessing plant offgas. Design Basis. Cryogenic distillation and pressurized cylinder storage.

Milestone. Start of Operation, early 1983. 
PNC: TOKAI (contd)

12. Bitumization Facility

Mission. Immobilize PNC's LLW.

Design Basis. $200 \mathrm{l} / \mathrm{hr}$.

13. Incinerator (Tokai)

Mission. Burn solid LLW.

Design Basis. $100 \mathrm{~kg} / \mathrm{hr}$.

14. Incinerator (0-Arai)

Mission. Burn solid LLW.

Design Basis. Three chambers--pyrolysis, combustion, after-burning.

15. Acid Digestion Facility (nonradioactive)

Mission. Confirm laboratory-scale results on acid digestion process for TRU wastes.

Design Basis. 200- $\ell$ tantalum digester vessel and ancillary equipment, glass-lined steel where necessary; capacity, $4-8 \mathrm{~kg} / \mathrm{hr}$.

History. In operation.

16. Plutonium Waste Treatment Facility

Mission. Prepare PNC TRU wastes for disposal. Design Basis. Acid digestion of chloride-containing wastes; incineration of other combustibles; mechanical volume reduction.

Milestone. Acid digestion facility to be operational by 1986. 
Radioactive Waste Management Center

No. 15, Mori Building

2-B-10, Toranomon

Minato-ku, Tokyo, 105

Japan

, Tel: (81) 3 504-1081

President

Ryoichi Takeuchi

Managing Director

Syunichi Murakoshi

Sponsors. Japanese industry, MITI and STA.

Mission. Promote the study of safe and rational operation of low-level radioactive waste disposal.

\section{STA (SCIENCE AND TECHNOLOGY AGENCY)}

Science and Technology Agency

2-2-1 Kasumi gaseki

Chiyoda-ku, Tokyo 100

Japan

Te1: (81) 35815271

Telex: 2226720 STASGD

Minister of State for Science and Technology

Deputy Minister

Administrative Vice Minister

Director-General, Nuclear

Safety Bureau

Director, Nuclear Materials

Regulation Division

Director, Nuclear Safety

Division

Director-General, Atomic

Takaaki Yasuta

Hiroko Hayashi

Takao Ishiwatari

Eijchi Tsuji

Tetsushi Kuramochi

Hiroyoshi Kurihari

Yukinobu Takaoka

Energy Bureau

Director, Nuclear Fuel

Division

Deputy Director

Fujio Sakauchi

M. Nomura 


\section{US EMBASSY - TOKYO}

American Embassy

10-5 Akasaka 1-chome

Minato-ku, Tokyo 107

Japan

US DOE Representative
Tel: (81) 35834121

Telex: 2422118

Billy D. Hill 

KOREA

(Republic of Korea) 


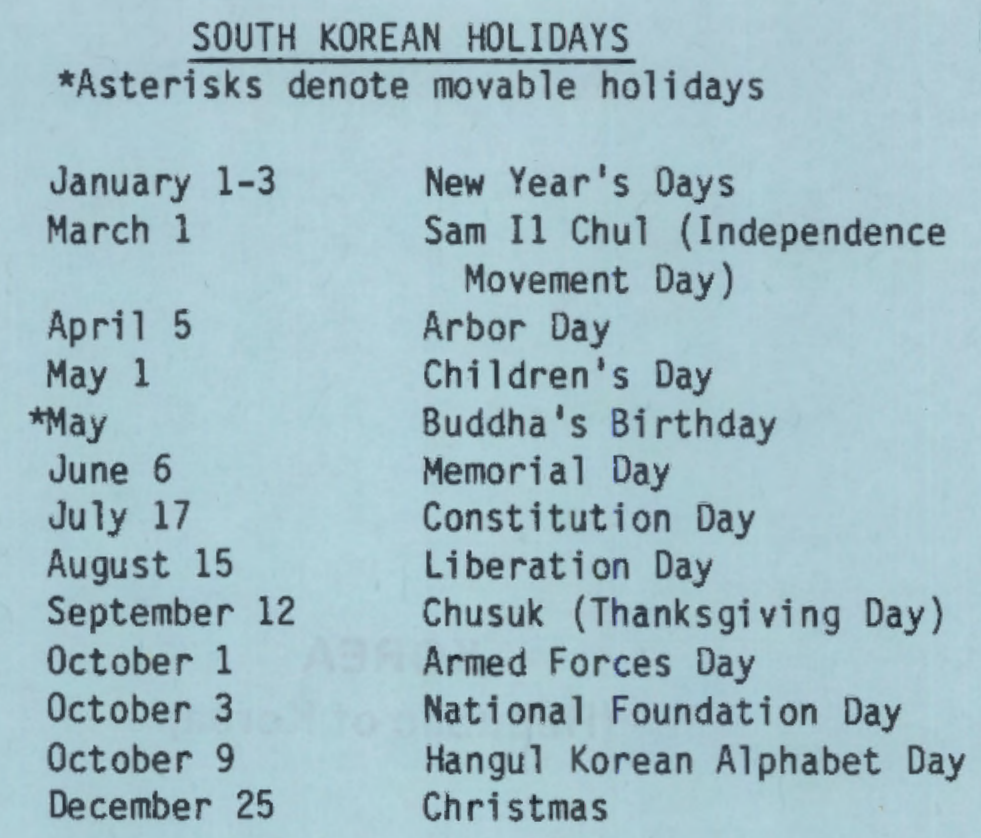

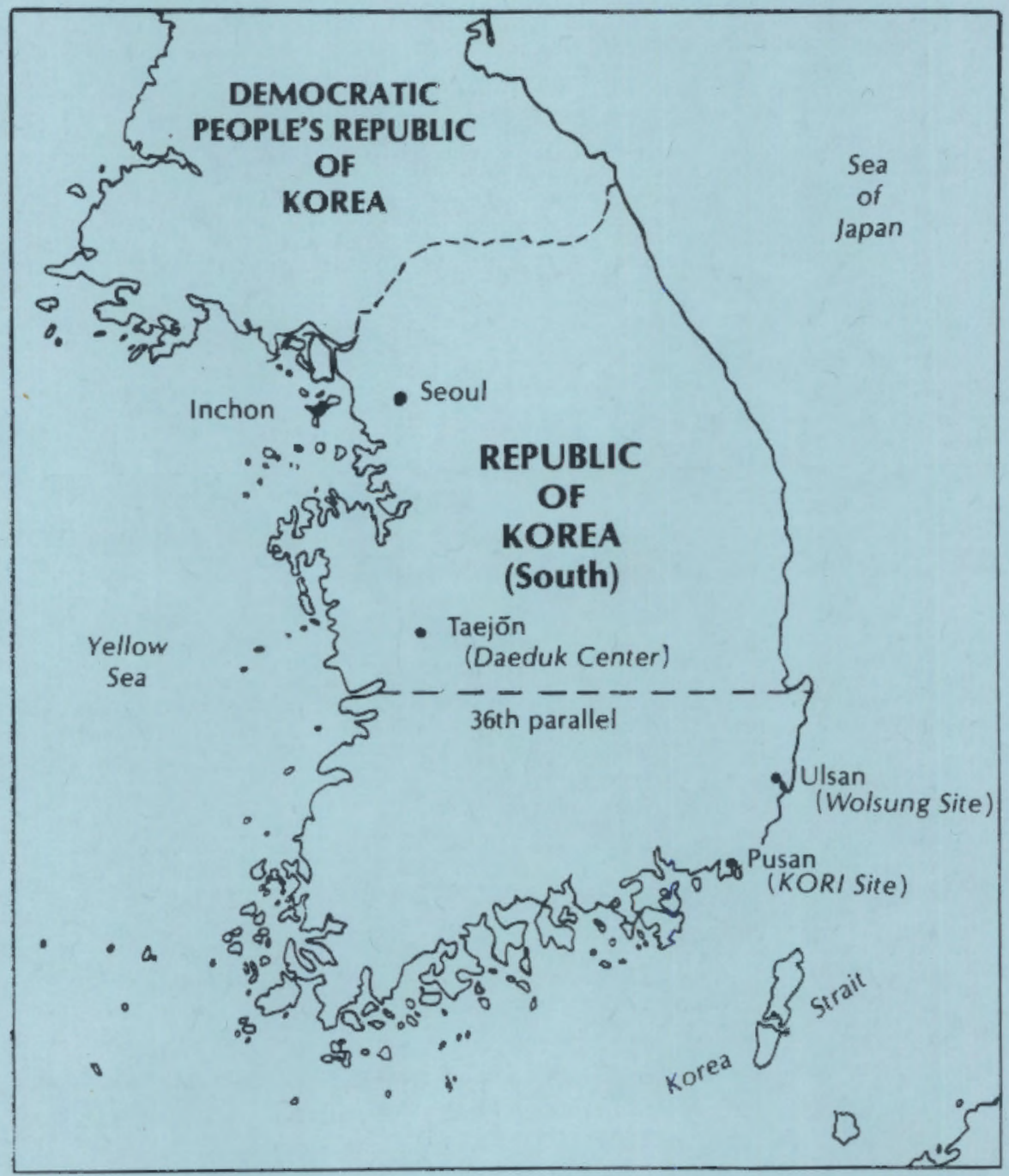




\section{KOREA (REPUBLIC OF KOREA)}

ENERGY

\begin{tabular}{|c|c|c|}
\hline Population & 1983 & 40 million \\
\hline Energy Demand & $\begin{array}{l}1980 \\
1985\end{array}$ & $\begin{array}{l}\text { 44.1 Mtoe } \\
\text { (73.6\% imported) } \\
58.1 \text { Mtoe } \\
\text { (7B.2\% imported) }\end{array}$ \\
\hline 0il Demand & 1980 & $\begin{array}{l}26.6 \text { Mtoe } \\
\text { (100\% imported) }\end{array}$ \\
\hline Electric Power Plant Capacity & 1990 & $\begin{array}{l}13.1 \text { GWe-- } \\
14.6 \% \text { nuclear } \\
54.8 \% \text { oil } \\
1.1 \% \text { hydro } \\
51 \% \text { nuclear }\end{array}$ \\
\hline lectric Power Production & 1986 & $\begin{array}{l}35.4 \text { TWh-- } \\
7.7 \% \text { nuclear } \\
1.5 \% \text { hydro } \\
57.1 \text { TWh-- } \\
46 \% \text { nuclear }\end{array}$ \\
\hline
\end{tabular}

NUCLEAR POWER

National Policy. Continue expansion in electric power capacity; reduce dependence on foreign oil by strong nuclear program; develop indigenous manufacturing capacity; develop FBR capability.

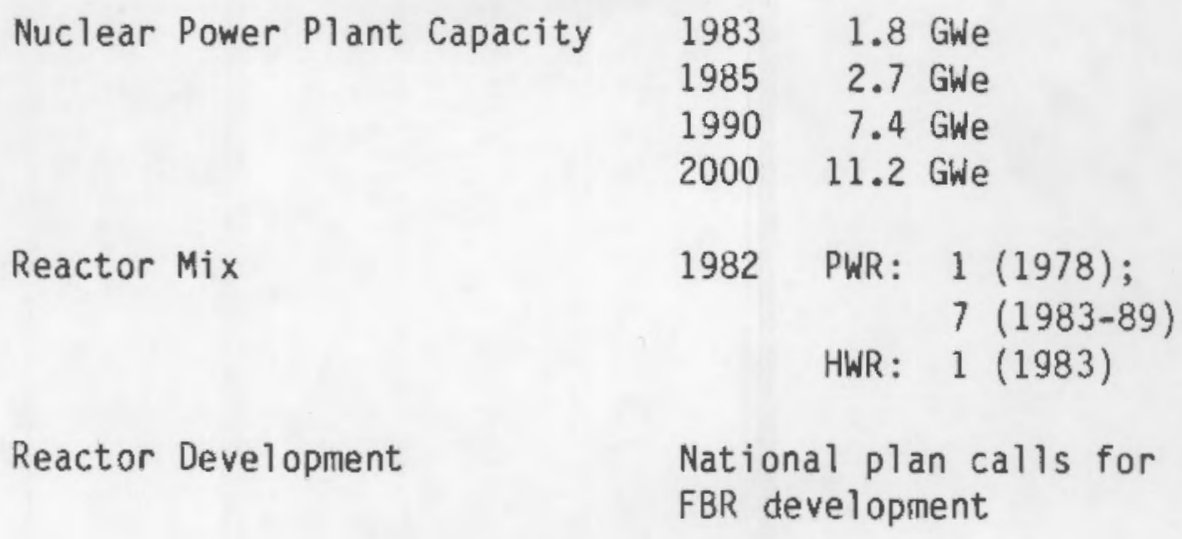


INDUSTRIAL FUEL CYCLE

Policy. Develop long-term contracts for fuel supplies;

holdings of foreign uranium resources; fabrication capability for PWP and CANDU fuels; "wait and see"-reprocessing and recycle of Pu to FBR, CANDU and LWRs.

Waste Management Strategy. Not yet defined.

Cumulative Spent Fue1

$1980 \quad 17 \mathrm{tU}$

Arisings

$1985 \quad 360 \mathrm{tU}$

$19901,450 \mathrm{tU}$

$2000 \quad 4,400 \mathrm{tU}$

Industrial-Scale Activities

1. Uranium milling--3 t ore/day pilot plant, under construction.

2. Uranium conversion, yellowcake to $\mathrm{UO}_{2}--150 \mathrm{tU} / \mathrm{a}$.

3. $\mathrm{UO}_{2}$ fuel fabrication--200 tU/a. Startup, 1988. 


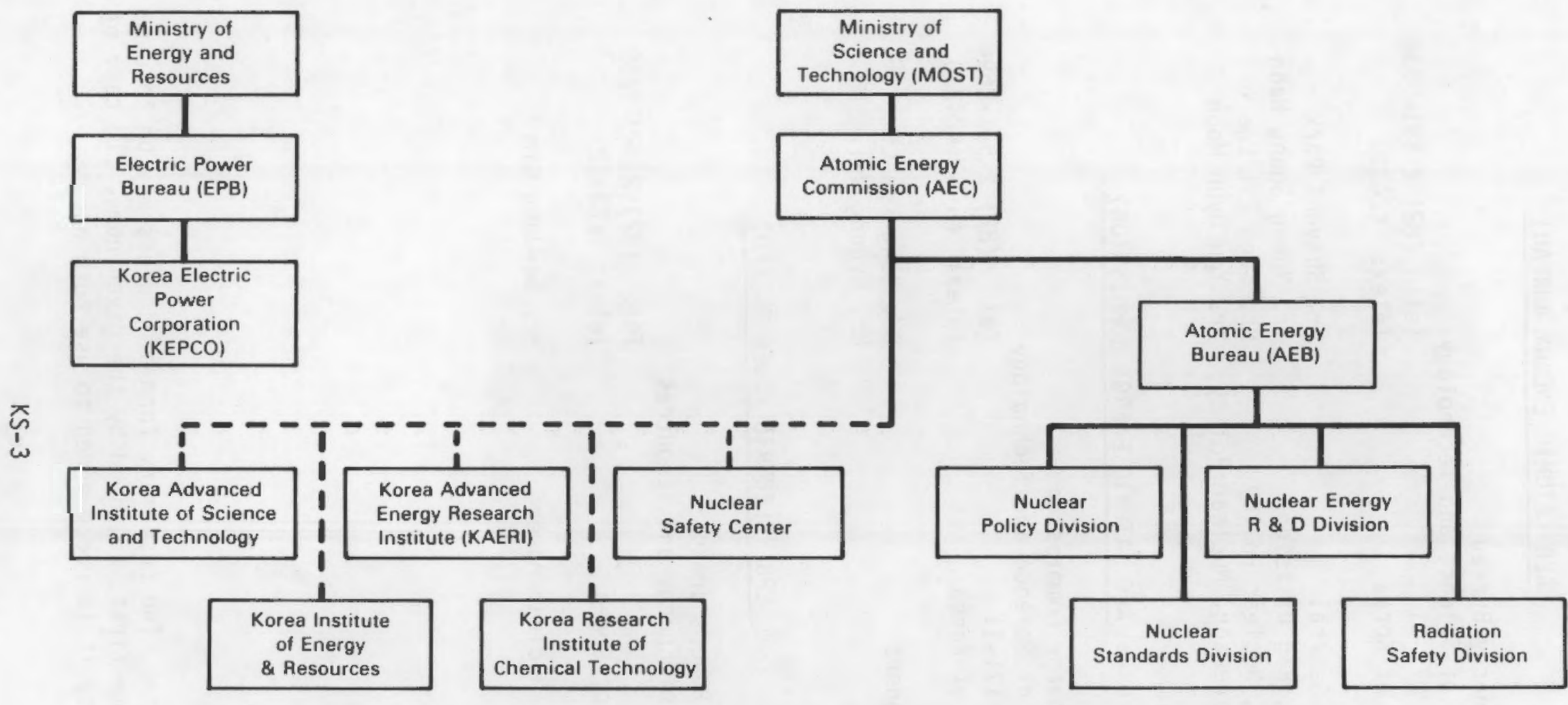

FIGURE KS-1. Partial Organization Chart for the Ministries of Science and Technology, and Energy and Resources 


\section{AEB (ATOMIC ENERGY BUREAU)}

Atomic Energy Bureau

Ministry of Science and Technology

GWACHEON 171-11

Tel: (82) 2 591-9038

Republic of Korea

Telex: $\mathrm{K} 24230$

Director-General

Mr. Shiyohl Park

Director, R\&D Division

Director, Nuclear Policy

Mr. Young Soung Hahn

Ass. Director, Nuclear Policy

Mr. Sang Tai Lee

$\mathrm{Mr}$. You Hyun Moon

\section{AEC (ATOMIC ENERGY COMMISSION)}

Atomic Energy Commission

Ministry of Science and Technology

GWACHEON 171-11

Tel: (82) 2 591-7694

Republic of Korea

Telex: MIOST K24230

Commissioners

Dr. Yong-Kyu Lim

Mr. Byoung Whie Lee

\section{EPB (ELECTRIC POWER BUREAU)}

Electric Power Bureau

Ministry of Energy and Resources

Seoul

Tel: (82) $2720-2369$

Republic of Korea

Telex: K23472

Director, Nuclear Power

Mr. Se-Jong Kim

Please Note: The customary form of address may be to use the surname first, followed by the given name. In case of uncertainty it is recommended to use both names. 


\section{KAERI (KOREA ADVANCED ENERGY RESEARCH INSTITUTE)}

Korea Advanced Energy Research Institute

Head Office and Seoul Research Center

P.0. Box 7, Cheong Ryang R1

Seoul 131

Republic of Korea

Tel: (82) 2 972-2081/9

President,

Vice President

Telex: KAERI K23241

Nuclear Policy

Mr. Jong Hee Cha

Dr. Pilsoon Han

Dr. Poong-Eil Juhn

Mission. Reactor engineering R\&D; radiation applications; basic nuclear research.

\section{KAERI： DAEDUK}

Daeduk Engineering Center, KAERI

P.0. Box 7

Daeduk Danji

Choongnam

Republic of Korea

Tel: (82) 42 822-6811

Republic of Korea

Telex: KAERI K 5553

Director

Dr. Pilsoon Han

Nuclear Fuel Cycle

(822-6821 Ext. 231)

Nuclear Fuel Cycle Process

Dr. Hyun-Soo Park

Development

Mission. R\&D on nuclear fuel cycle technology

- Fuel fabrication

- Uranium ore processing and conversion

- Radioactive waste management

- Post-irradiation examination.

Please Note: The customary form of address may be to use the surname first, followed by the given name. In case of uncertainty it is recommended to use both names. 


\section{KOPEC (KOREA POWER ENGINEERING COMPANY)}

Korea Power Engineering Co., Inc.

P.O. Box 109

YEOEUIDO

Seoul 150

Tel: (82) 2 783-7606

Republic of Korea

Telex: KOPEN K22562

Vice President, Engineering Mr. Kui Yong Park

KEPCO (KOREA ELECTRIC POWER CORPORATION)

Korea Electric Power Corporation

87, Samseong-Dong

Gangnam-Ku

Seoul,

Tel: (82) $2562-9926$

Republic of Korea

Telex: Kelecco K24287, K28350

Manager, Nuclear Fuel

Mr. Chang Kook Yang

\section{KOREA NUCLEAR FUEL COMPANY}

Korea Nuclear Fuel Company, Ltd.

P.0. Box 7

Daeduk Danji, Daejeon

Choongnam 300

Tel: (82) $42822-6820 / 9$

Republic of Korea

Telex: $\mathrm{K} 5553$

President

Dr. Pilsoon Han

Please Note: The customary form of address may be to use the surname first, followed by the given name. In case of uncertainty it is recommended to use both names. 
MEXICO 


\begin{tabular}{|c|c|}
\hline January 1 & New Year's Day \\
\hline January 6 & Three Kings Day \\
\hline February 5 & Promulgation of Constitution \\
\hline March 21 & Birthday of Benito Juarez \\
\hline \#arch/April & Maundy Thursday \\
\hline *March/April & Good Friday \\
\hline May 1 & Labor Day \\
\hline May 5 & Victory of Gen. Zaragosa \\
\hline September 16 & Independence Day \\
\hline October 12 & Columbus Day \\
\hline November 2 & Al1 Souls' Day \\
\hline November 20 & Anniv. of the Revolution \\
\hline December 3 & Immaculate Conception \\
\hline December 12 & Our Lady of Guadalupe's Day \\
\hline December 25 & Christmas \\
\hline
\end{tabular}

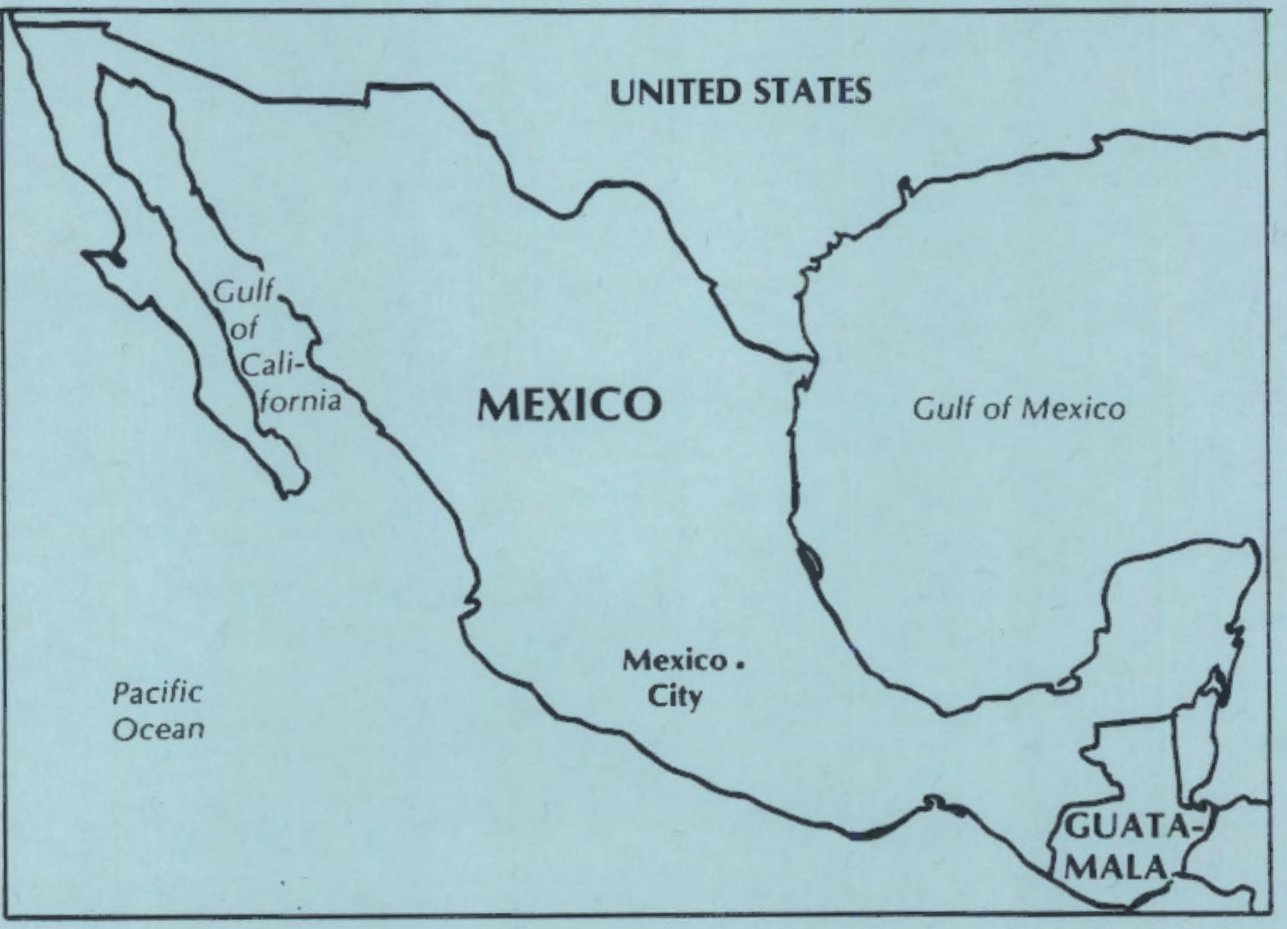




\section{MEXICO}

ENERGY

\begin{tabular}{|c|c|c|}
\hline Population & 1982 & 76 million \\
\hline Energy Demand & 1979 & 77 Mtoe \\
\hline Electric Power Plant Capacity & 1983 & $18 \mathrm{GHe}$ \\
\hline Electric Power Production & 1978 & $\begin{array}{l}53 \text { TWh-- } \\
69 \% \text { oil \& gas } \\
30 \% \text { hydro } \\
1 \% \text { geothermal } \\
64.2 \text { TWh }\end{array}$ \\
\hline
\end{tabular}

NUCLEAR POWER

National Policy. Future is cloudy; ambitious plans to use extensive uranium reserves in domestic nuclear power production have been thwarted by national economic problems, cost overruns with nuclear plant construction, etc.

$\begin{array}{lll}\text { Nuclear Power Plant Capacity } & 1986 & 0.7 \mathrm{GWe} \\ & 1990 & 1.3 \mathrm{GWe} \\ & 2000 & 1.3 \mathrm{GWe}\end{array}$

Reactor Mix 1982 BWR: 2 (1984-85)

INDUSTRIAL FUEL CYCLE

Policy. Future is cloudy; plans for pilot reprocessing and waste management facilities have been dropped, but uranium mining/milling and fuel fabrication work continues.

Waste Management Strategy. Not announced.

Cumulative Spent Fuel $\quad 1990 \quad 100$ tU

Arisings (LWR) $2000 \quad 500 \mathrm{tU}$ 
ORGANIZATION

All nuclear activities controlled by federal government through:

- National Atomic Energy Commission (CNEA)

- URAMEX--fuel cycle activities, including exploitation of uranium resources

- National Nuclear Research Institute (ININ)--R\&D

- National Commission of Nuclear Security and Safeguards (CNSNS)--safety R\&D and licensing of nuclear facilities.

\section{CNSNS (NATIONAL COMMISSION OF NUCLEAR}

$$
\text { SECURITY AND SAFEGUARDS) }
$$

Comisión Nacional de Seguridad

Nuclear y Salvaguardias

Av. Insurgentes Sur No. 1806

Colonia Florida, Delegación Alvaró obregón

01030 Mexico, D.F. (Mexico City) Tel:

(52) 55341401

\section{ININ (NATIONAL NUCLEAR RESEARCH INSTITUTE)}

Instituto Nacional de Investigaciones

Nucleares

Benjamin Franklin No. 161

Colonia Condesa Delagacion Cuauh'temóc

06140 Mexico, D.F. (Mexico City) Te1:

(52) 52710547

Director General

Manager, Radiological Safety

R. Bello

Jose Roberto Martinez

Salinas

(ININ's Salazar Plant is located about $35 \mathrm{~km}$ from Mexico City. Mail should be addressed to the Mexico City headquarters.) 
Mexico

\section{URAMEX}

Uranio Mexicano

Insurgentes Sur No. 1079

Colonia Noche Buena Delegación Benito Juárez

03720 Mexico, D.F. (Mexico City) Tel: (52) 55637752

Director General

Dr. Alberto Escofet

Director, Fuel Cycle

Dr. Carlos Graef Fernandez 

NETHERLANDS 


$\begin{array}{ll}\text { *Asterisks } & \text { DUTCH HOLIDAYS } \\ \text { January 1 } & \text { New Year's Day } \\ \text { *March/April } & \text { Good Friday } \\ \text { *March/April } & \text { Easter Monday } \\ \text { April 30 } & \text { Queen's Birthday } \\ \text { *May } & \text { Ascension Day } \\ \text { *May } & \text { Whit Monday } \\ \text { December 25-26 } & \text { Christmas }\end{array}$

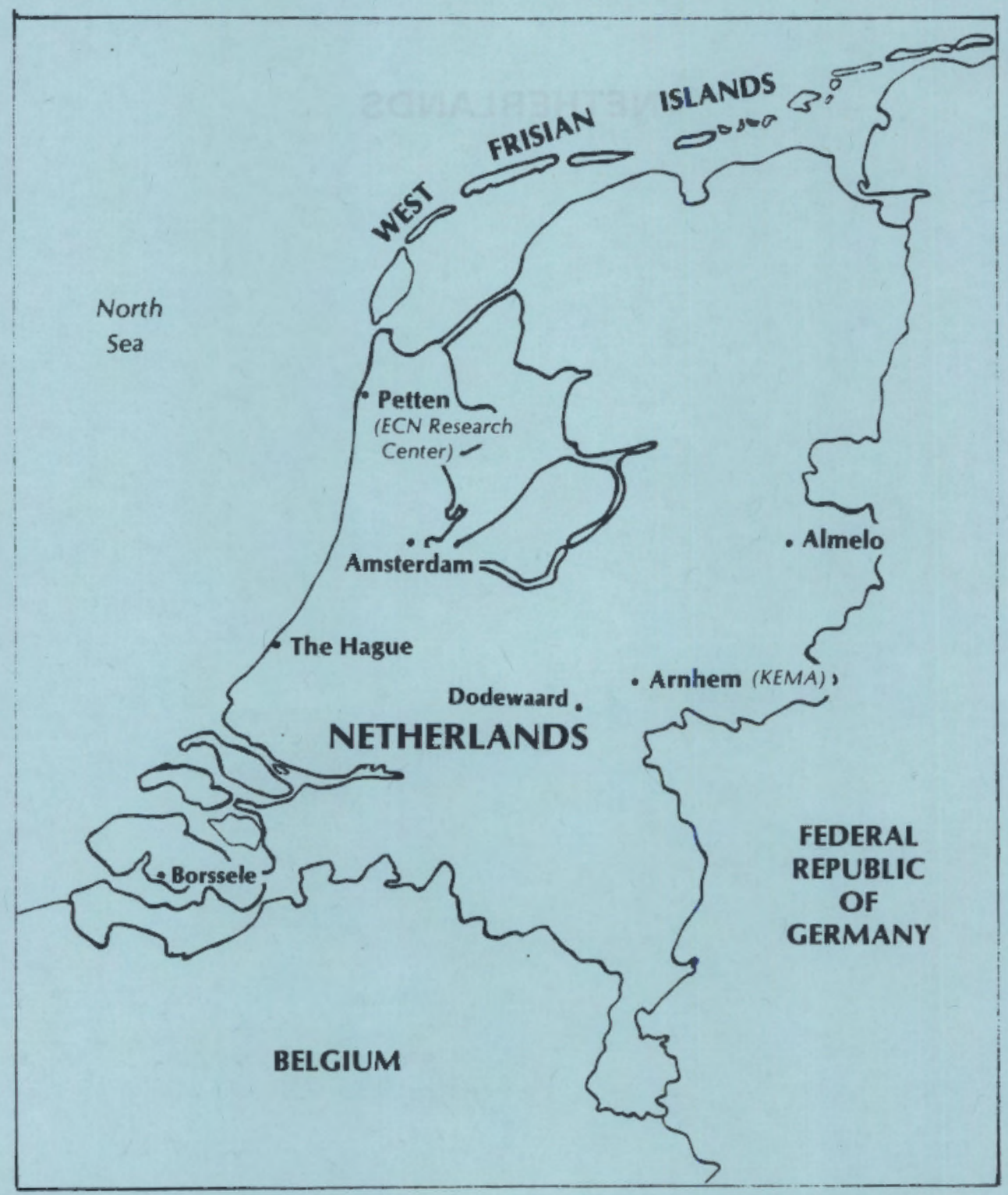




\section{NETHERLANDS}

\section{ENERGY}

\begin{tabular}{|c|c|c|}
\hline Population & 1980 & 14.1 million \\
\hline Energy Demand & 1980 & 65.5 Mtoe \\
\hline 0i1 Demand & 1979 & $\begin{array}{l}40.1 \text { Mtoe } \\
\text { (>90\% imported) }\end{array}$ \\
\hline Electric Power Plant Capacity & 1980 & 17 GWe \\
\hline Electric Power Production & 1980 & $\begin{array}{l}64.8 \text { Th-- } \\
6.5 \% \text { nuclear } \\
15.2 \% \text { solid fuels } \\
38.3 \% \text { petroleum } \\
\text { products } \\
39.7 \% \text { gas } \\
53 \text { TWh-- } \\
6.8 \% \text { nuclear }\end{array}$ \\
\hline
\end{tabular}

NUCLEAR POWER

National Policy. The government favors expansion of nuclear power capacity, but faces public opposition; the utilities want three more plants $(1,000$ MWe each). Polls in a lengthy series of lightly attended public meetings have shown a large number of those attending as being opposed to nuclear power.

$\begin{array}{lll}\text { Nuclear Power Plant Capacity } & 1982 & 0.5 \text { GWe } \\ & 2000 & 0.5 \text { GWe } \\ \text { Reactor Mix } & 1982 & \text { BWR: } 1(1969) \\ & & \text { PWR: } 1(1973) \\ \text { Reactor Development } & & \text { Participation in FRG } \\ & \text { Kalkar SNR-300 FBR project }\end{array}$


Netherlands

INDUSTRIAL FUEL CYCLE

Policy. Use foreign services (fuel fabrication, reprocessing).

Waste Management Strategy. LLW--resume ocean dumping, if political climate improves; HLW--develop interim storage capability and evaluate geologic disposal on land, in a salt dome under the North Sea, or under the seabed.

$\begin{array}{ccc}\text { Cumulative Spent Fuel } & 1980 & 103 \mathrm{tU} \\ \text { Arisings (LWR) } & 1985 & 190 \mathrm{tU} \\ & 1990 & 270 \mathrm{tU} \\ & 2000 & 420 \mathrm{tU}\end{array}$

\section{INTERNATIONAL RELATIONSHIPS}

1. Member state of European Communities, OECD/NEA, IAEA.

2. Partnership with FRG and Belgium in SNR-300 FBR.

3. Partnership with FRG and UK in Urenco (uranium enrichment consortium).

ORGANIZATION

1. Overall control of nuclear matters is exercised by the Ministries of Economic Affairs, Public Health and Environmental Control, and Social Affairs; Parliament approves decisions of the ministries.

2. Waste management $R \& D$ is integrated under a program called ILONA, directed by a policy committee which includes representatives from the ministries and the Netherlands energy research center at Petten (ECN), ILONA is partly financed by the CEC Indirect Action Program. Supporting R\&D studies are conducted at:

- ECN (in situ experiments at the Asse Mine in Germany--borehole convergence at elevated temperatures) 
- Delft University, Department of Mining (stress cracking in gallery walls, repository design, borehole closure, safety assessment)

- Utrecht University, Department of Earth Sciences (salt rheology under repository conditions).

\section{ECN (NETHERLANDS ENERGY RESEARCH FOUNDATION)}

Stichting Energieonderzoek Centrum Nederland

Scheveningse Weg 112

P.0. Box 80404

2508 GK The Hague

The Netherlands

Tel: (31) 70514581

Telex: 31459

Chairman, Governing Board

G. W. zen Stein Callensels

Chairman, ECN Scientific

Prof. J. van Loef

Advisory Center

Chairman, Program Committee Prof. P. de Wolff

of the Energy Study Center

Mission. Organize and sponsor energy research and development.

Sponsor. Federal government.

\section{ECN: PETTEN}

Research Centre

ECN Netherlands Energy Research Foundation

P.0. Box 1

1755 ZG Petten

The Netherlands

Tel: (31) 22466262

Telex: 57211 Reacp NL 
Netherlands

\section{ECN: PETTEN (contd)}

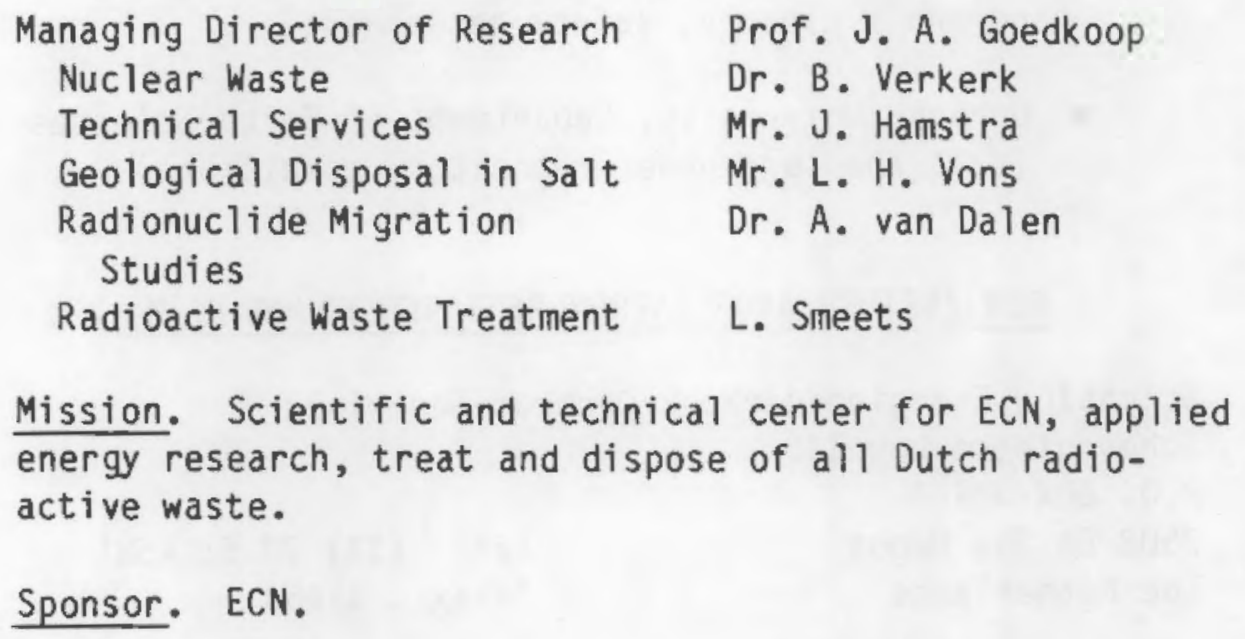

Mission. Scientific and technical center for ECN, applied energy research, treat and dispose of all Dutch radioactive waste.

Sponsor. ECN.

Waste Management R\&D. Geologic waste isolation--salt dome repositories (conceptual design, thermal studies, and radionuclide migration), seabed disposal, sea disposal of ${ }^{85} \mathrm{Kr}$, decontamination of large components.

\section{GEOLOGICAL SURVEY OF THE NETHERLANDS}

Geological Survey of the Netherlands

Nieuwe Gracht 13

Postbus 157

2000 AD Haarlem

The Netherlands

Tel: (31) 23319362

Telex: 71105 geold

Dr. H. M. van Montfrans

\section{KEMA (RESEARCH AND TESTING ELECTROCHEMICAL} MATER IALS COMPANY)

N.V. Tot Keuring van Elektrotechnische

Materialen Arnhem

Utrechtseweg 310

P.0. Box 9035

6800 ET Arnhem

Tel: (31) 85457057

The Netherlands

Telex: 45016 Kema $n 1$ 
Netherlands

KEMA (RESEARCH AND TESTING ELECTROCHEMICAL MATERIALS COMPANY) (contd)

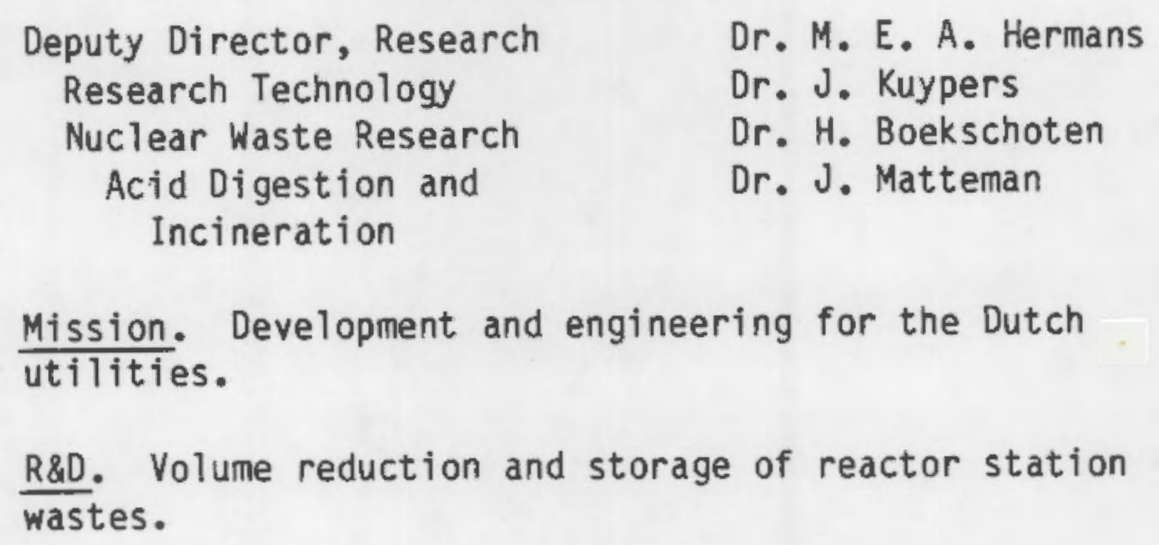

Mission. Development and engineering for the Dutch utilities.

R\&D. Volume reduction and storage of reactor station wastes.

\section{Facilities}

Acid Digestion Pilot Plant

Mission. Explore process parameters and establish equipment operability.

MINISTRY OF HOUSING, PHYSICAL PLANNING AND ENVIRONMENT

Ministry of Housing, Physical Planning and Environment

P.0. Box 5811

Koopmansstraat 1

2280 HV Rijswijk

Tel: (31) 70949505

The Netherlands

Telex: 32691

Deputy Chief

Director, Radiation

Dr. J. Ch. Cornelis

Protection

Dr. J. L. Baas 



\section{PAKISTAN}




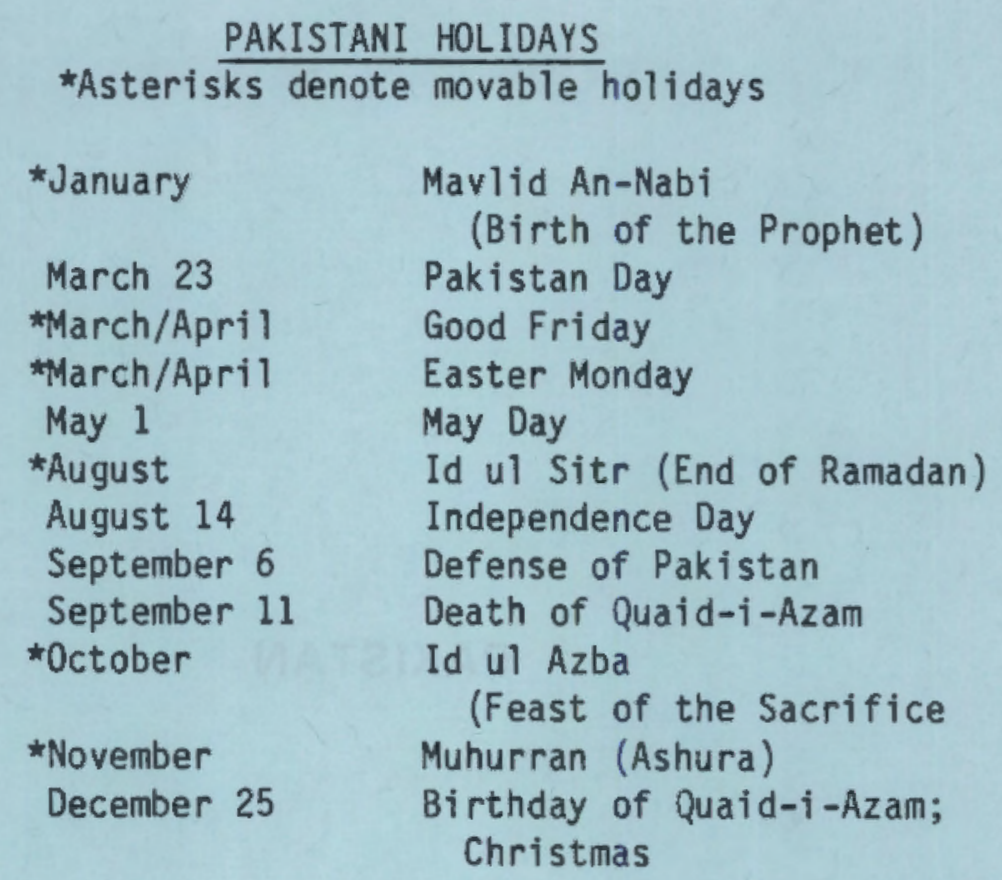

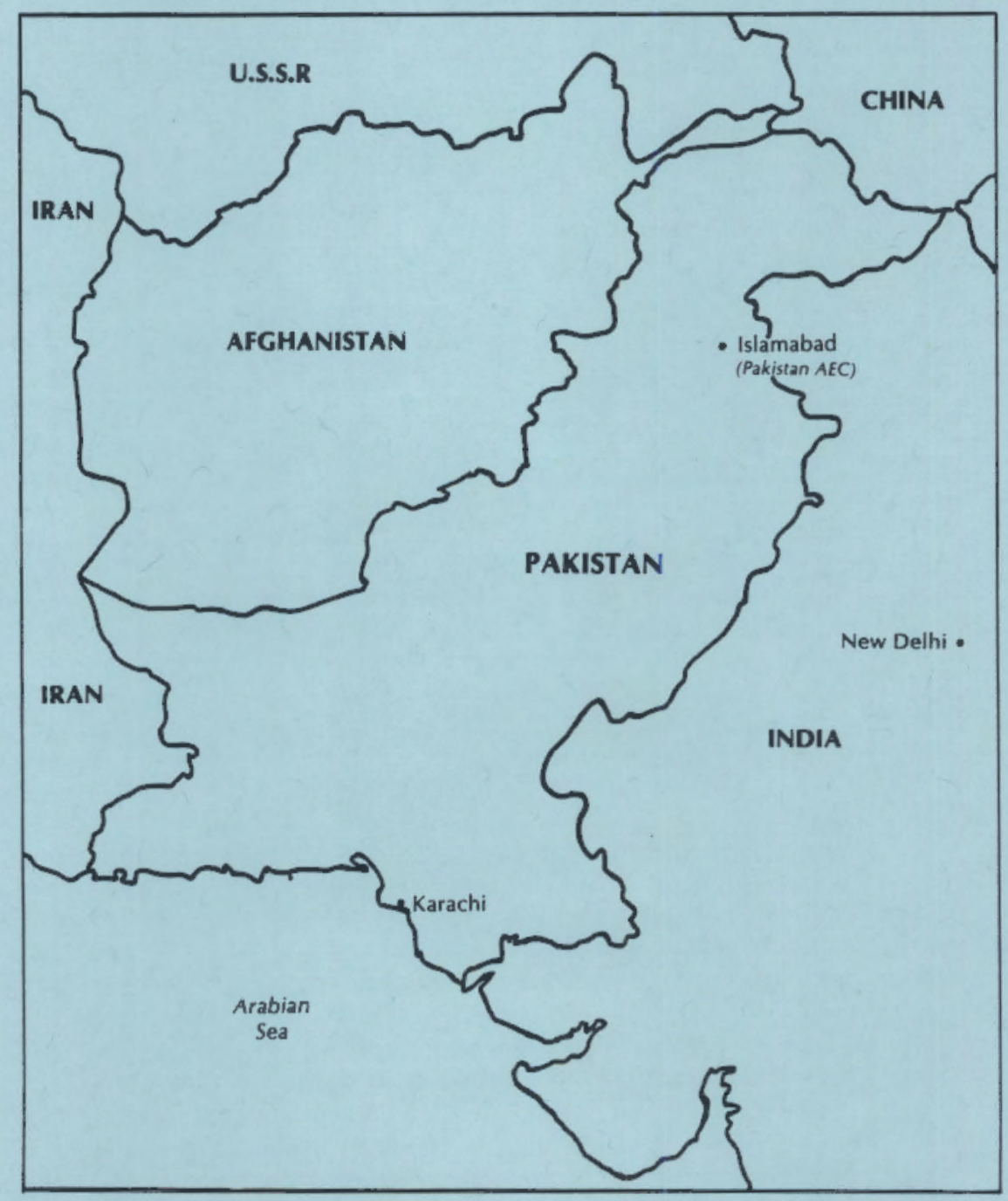




\section{PAKISTAN}

ENERGY

\begin{tabular}{|c|c|c|}
\hline Population & 1980 & 81.5 million \\
\hline Energy Demand & $\begin{array}{l}1980 \\
1981-82\end{array}$ & $\begin{array}{l}12.4 \text { Mtoe } \\
0.3 \% \text { nuclear } \\
42.6 \% \text { gas } \\
35.0 \% \text { oil } \\
17.2 \% \text { hydro } \\
4.9 \% \text { coal }\end{array}$ \\
\hline 0il Demand & 1980 & $90 \%$ imported \\
\hline $\begin{array}{l}\text { Electric Power Plant } \\
\text { Capacity }\end{array}$ & $1981-82$ & 4 GWe \\
\hline Electric Power Production & $1981-82$ & $\begin{array}{l}12.6 \text { TWh-- } \\
56 \% \text { hydro }\end{array}$ \\
\hline
\end{tabular}

NUCLEAR POWER

National Policy. Provide up to $50 \%$ of electrical power supply with nuclear.

$\begin{array}{lcc}\text { Nuclear Power Plant } & 1982 & 0.1 \text { GWe } \\ \text { Capacity (LWR and HWR) } & 1995 & 1.1 \text { GWe } \\ & 1982 & \text { HWR (CANDU): } \\ \text { Reactor Mix } & & 1 \text { (1972) }\end{array}$

PWR: 1 (1993)

\section{INDUSTRIAL FUEL CYCLE}

Policy. Develop complete domestic fuel cycle: uranium mining and milling; enrichment; fuel fabrication; reprocessing.

$\begin{array}{llr}\text { Cumulative Spent Fuel } & 1980 & 49 \mathrm{tU} \\ \text { Arisings } & 1985 & 110 \mathrm{tU} \\ & 1990 & 170 \mathrm{tU} \\ & 2000 & 440 \mathrm{tU}\end{array}$




\section{ORGANIZATION}

1. Pakistan Atomic Energy Commission--control of nuclear matters.

2. Pakistan Institute of Science and Technology (Rawalpindi)--fuel cycle R\&D, including lab-scale reprocessing facility.

\section{PAKISTAN ATOMIC ENERGY COMMISSION}

Pakistan Atomic Energy Commission

P.0. Box 1114

Islamabad, Pakistan

Chai rman

Dr. Munir Ahmad Khan

PK-2 
SOUTH AFRICA (Republic of) 


\section{SOUTH AFRICAN HOLIDAYS}

*Asterisks denote movable holidays

January 1

*April

* March/April

*March/Apri 1

*May

May 31

October 10

*December

December 25-26
New Year's Day

Founder's Day

Good Friday

Family Day

Ascension Day

Republic Day

Kruger Day

Covenant Day

Christmas

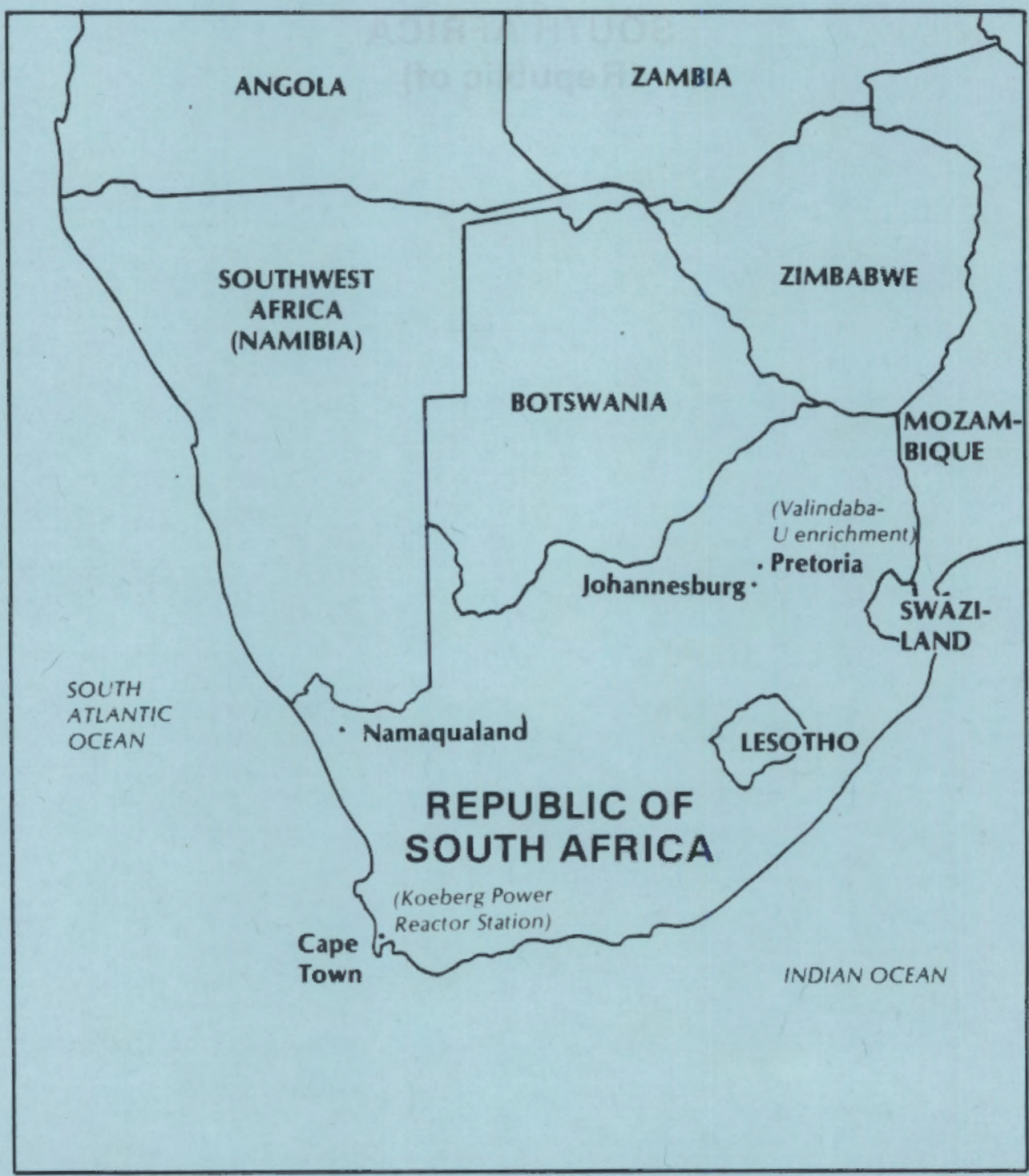




\section{SOUTH AFRICA}

\section{ENERGY}

$\begin{array}{lcc}\text { Population } & 1980 & 28 \text { million } \\ \text { Energy Demand } & 1980 & 85.7 \text { Mtoe } \\ \text { 0il Demand } & & 100 \% \text { imported } \\ \text { Electric Power Plant Capacity } & 1982 & 20 \mathrm{GWe} \\ & 2000 & 70 \mathrm{GWe}-- \\ 5 \% \text { nuclear }\end{array}$

National Policy. Expand electric power production capacity chiefly through coal-burning plants, but develop small nuclear capability.

$\begin{array}{lll}\text { Nuclear Power Plant Capacity } & 1984 & 0.9 \text { GWe } \\ & 1990 & 1.8 \text { GWe } \\ & 2000 & 2.8 \text { GWe }\end{array}$

Reactor Mix

1982 PWR: $2(1984-85)$

INDUSTRIAL FUEL CYCLE

Policy. Produce and export uranium (7,000-8,000 tU/a); develop enrichment capability (200-300 tSWU/a). No current plans for fuel fabrication or reprocessing.

Waste Management Strategy. Store reactor wastes at the reactor until a disposal site is operational (1994); build a repository for HLW or spent fuel in an area 800-900 miles west of Pretoria, south of the Kalahari Desert.

$\begin{array}{ccr}\text { Cumulative Spent Fuel } & 1985 & 40 \mathrm{tU} \\ \text { Arisings (LWR) } & 1990 & 310 \mathrm{tU} \\ & 2000 & 1,200 \mathrm{tU}\end{array}$


South Africa

ORGANIZATION

The Atomic Energy Corporation of South Africa (governmentowned) controls nuclear matters through:

- Atomic Energy Board--regulating and licensing

- Nuclear Development Corporation of South Africa (NUCOR)--R\&D, including waste disposal and repository work

- Uranium Enrichment Corporation of South Africa (UKOR).

\section{ATOMIC ENERGY CORPORATION}

Atomic Energy Corporation of South Africa Ltd. South African Atomic Energy Board

Private Bag X256

Pretoria 0001

Tel: (27) 12 21-3311

South Africa

Telex: 3-0253 SA

Chairman

J. W. de Villiers

\section{ESCOM (ELECTRICITY SUPPLY COMMISSION)}

Electricity Commission of South Africa

P.0. Box 1091

Johannesburg $2000 \quad T e 1:$ (27) 11 800-8111

South Africa

Telex: 4-24 481 SA

Chairman

Jan H. Smith

Senior General Manager

I. D. van der Walt 


\section{NUCOR (NUCLEAR DEVELOPMENT CORPORATION)}

Nuclear Development Corporation of South Africa (Pty), Ltd.

Private Bag X256

Pretoria 0001

South Africa

Tel: (27) 12 21-3311

Telex: 3-0253 SA

Managing Director

Dr. J. P. Hugo

Geology

P. D. Toens

Chemical Operations

H. J. Van der Westhuizen

UKOR/UCOR (URANIUM ENRICHMENT CORPORATION)

Uranium Enrichment Corporation

of South Africa (Pty), Ltd.

P.0. Box 4587

Pretoria 0001

South Africa

Tel: (27) 12 296-9111

Telex: 30805 SA

Managing Director

W. L. Grant 



$\begin{array}{ll}\text { *Asterisks denote movable } & \text { HOLIDAYS } \\ \text { January } 1 & \text { New Year's Day } \\ \text { January } 6 & \text { Epiphany } \\ \text { *March/Apri1 } & \text { Maundy Thursday } \\ \text { *March/April } & \text { Good Friday } \\ \text { May 1 } & \text { Labor Day } \\ \text { *June } & \text { Corpus Christi Day } \\ \text { July 25 } & \text { St. James Day } \\ \text { August } 15 & \text { Assumption Day } \\ \text { October } 12 & \text { Columbus Day } \\ \text { November } 1 & \text { All Saints' Day } \\ \text { December } 8 & \text { Immaculate Conception } \\ \text { December } 25 & \text { Christmas }\end{array}$

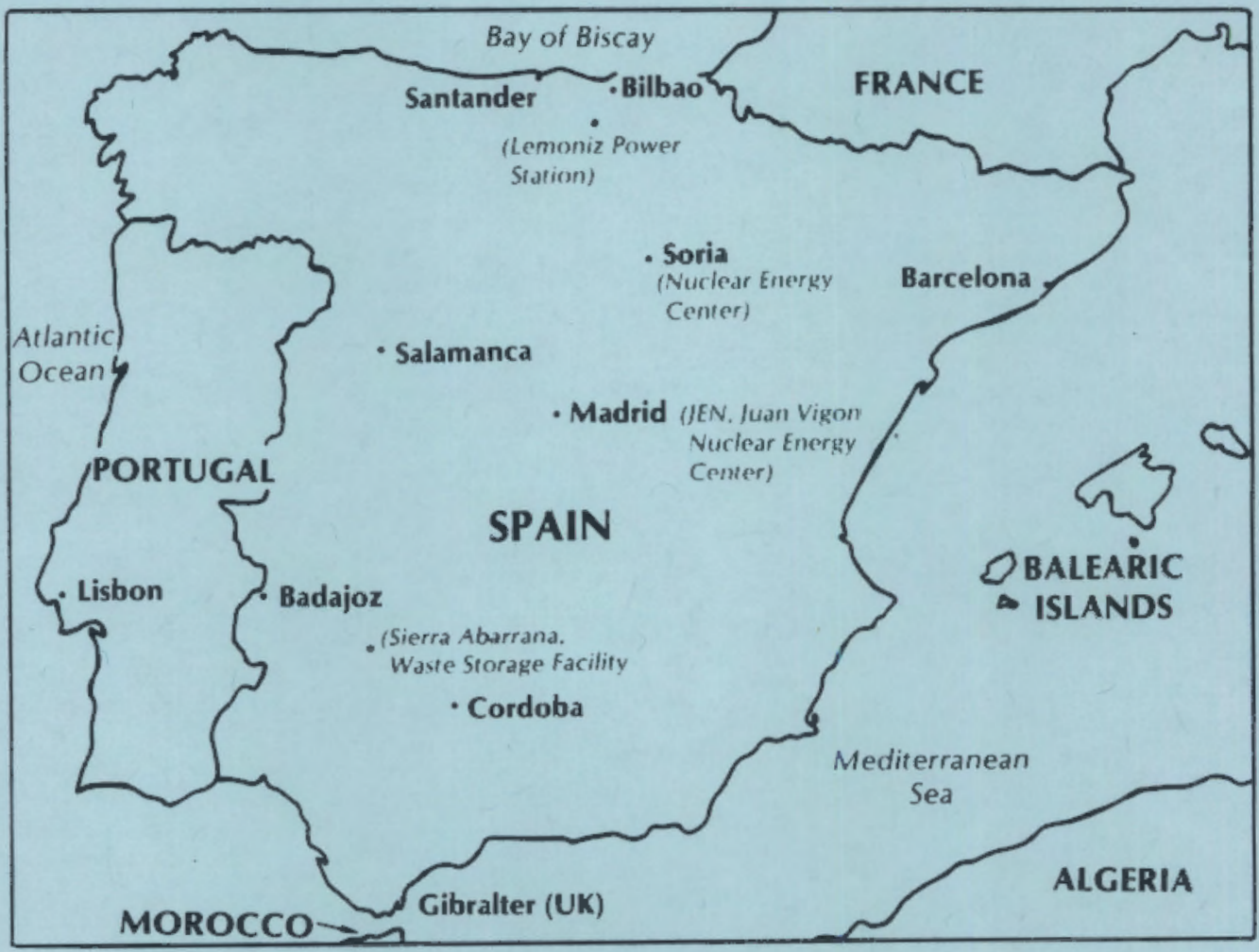


$\underline{\text { SPAIN }}$

ENERGY

\begin{tabular}{|c|c|c|}
\hline Population & 1982 & 38.3 million \\
\hline Energy Demand & $\begin{array}{l}1982 \\
1990\end{array}$ & $\begin{array}{r}64.3 \text { Mtoe } \\
106.5 \text { Mtoe }\end{array}$ \\
\hline Oil Demand & $\begin{array}{l}1982 \\
1990\end{array}$ & $\begin{array}{l}48.2 \text { Mtoe } \\
\text { (96\% imported) } \\
48.1 \text { Mtoe } \\
\text { (94.7\% imported) }\end{array}$ \\
\hline Electric Power Plant Capacity & $\begin{array}{l}1982 \\
1990\end{array}$ & $\begin{array}{l}33.5 \text { GWe } \\
44.2 \text { GWe }\end{array}$ \\
\hline Electric Power Production & 1982 & $\begin{array}{l}114.1 \text { Th-- } \\
7.68 \% \text { nuclear } \\
28.66 \% \text { oil \& gas } \\
39.65 \% \text { solid fuels } \\
24.01 \% \text { hydro/ } \\
\text { geothermal }\end{array}$ \\
\hline
\end{tabular}

NUCLEAR POWER

Nuclear Power Plant Capacity $1983 \quad 2.0$ GWe

$1990 \quad 7.5$ GWe

$2000 \quad 10.2$ GWe

Reactor Mix

1982 GCR: 1 (1972)

PWR: 2 (1969,1981)

$8(1983-90)$

BWR: 1 (1971)

3 (1984-88)

Reactor Development: breeder and fusion R\&D

INDUSTRIAL FUEL CYCLE

Policy. No domestic reprocessing and no further contracts for foreign reprocessing. 
Waste Management Strategy. Store spent fuels at the reactor sites for 10 years, then transfer to AFR engineered (dry) storage facility until geologic repository is ready to receive "high-level wastes" (spent fuels); locate AFR and fuel encapsulation facility at repository site (salt dome or granite formation). Shallow-land burial of LLW in trenches. Institutional radioactive wastes are currently placed in a worked-out uranium mine.

Cumulative Spent Fuel

$\begin{array}{rr}1980 & 176 \mathrm{tU} \\ 1985 & 490 \mathrm{tU} \\ 1990 & 1,300 \mathrm{tU} \\ 2000 & 3,400 \mathrm{tU}\end{array}$

Industrial-Scale Activities

1. Uranium mining and milling: $200 \mathrm{tU} / \mathrm{a}$.

2. Uranium enrichment: $11.1 \%$ interest in Eurodif.

3. Fuel fabrication: $200 \mathrm{tU} / \mathrm{a}$.

4. AFR spent fuel storage: $1500 \mathrm{tU}$.

\section{CSN (COUNCIL OF NUCLEAR SAFETY)}

Consejo de Seguridad Nuclear

Paseo de la Castellana 135

Madrid 16, Spain

Tel: (34) 14505062

Telex: 49051 CSNM E

President

Francisco Pascual

Counselor

Prof. L. Guitiérrez Jodra

Mission. Assess the safety of nuclear technology and environmental protection; perform plant inspections and issue licenses. 
ENUSA (NATIONAL FUEL CYCLE COMPANY)

Empresa Nacional del Uranio S.A. Santiago Rusiñol 12

Madrid 3, Spain

Tel: (34) 1 233-6207

Telex: 43402 URAN E

Director General

Manuel Isla

Mission. Supply fuel cycle services except waste management and disposal (uranium mining and milling; nuclear fuel) to all Spanish nuclear power plants.

\section{JEN (NUCLEAR ENERGY COMMISSION)}

Junta de Energía Nuclear

Avenida Complutense 22

Ciudad Universitaria

Tel: (34) 1 224-1200

Madrid 3, Spain

Telex: 23555 JUVIG E

President

General Director

Martín Gallego

Gonzalo Madrid

Director, Radioactive Waste

Baldomero López-Pérez

Waste Treatment

V. González

Spent Fuel Storage

A. Uriarte

Waste Storage

A. Martínez Martínez

Mission. R\&D: nuclear fuel cycle, nuclear reactor technology, waste treatment, and terminal storage.

JUAN VIGON NATIONAL NUCLEAR ENERGY CENTER

Centro Nacional de Energía

Nuclear Juan Vigon

Avenida Complutense 22

Madrid 3, Spain

MINISTRY OF INDUSTRY AND ENERGY

Minister

Director General of Energy

Carlos Solchaga

María del Carmen Mestre 



\section{SWEDEN}


SWEDISH HOLIDAYS

*Asterisks denote movable holidays

January 1

January 6

*March/April

*March/April

May 1

*May/June

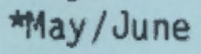

*June

* November

December 25-26
New Year's Day

Epiphany

Good Friday

Easter Monday

Labor Day

Ascension Day

Whit Monday

Midsummer Eve Day

All Saints' Day

Christmas

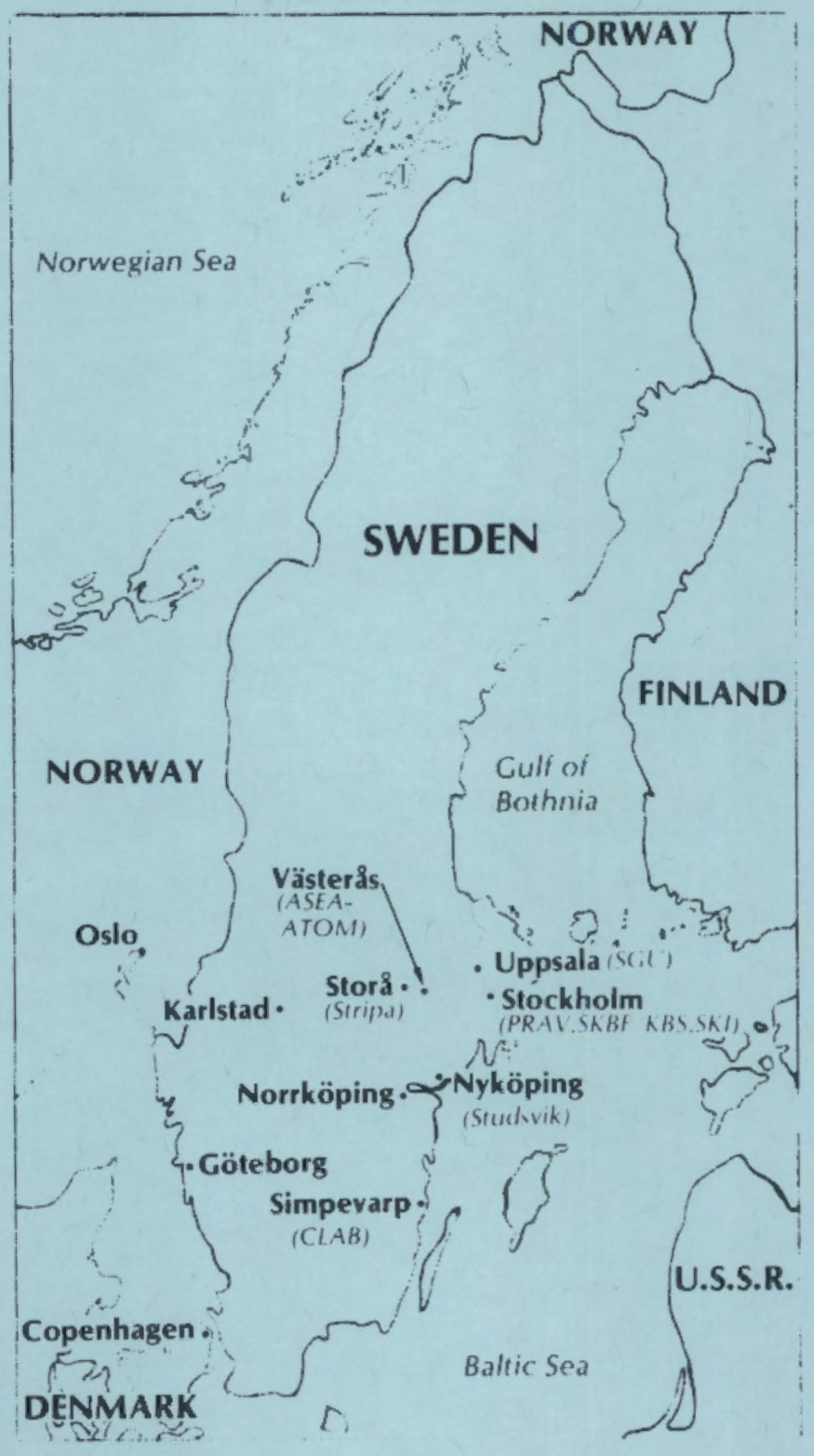




\section{SWEDEN}

ENERGY

\begin{tabular}{|c|c|c|}
\hline Population & 1980 & 8.3 million \\
\hline Energy Demand & 1980 & $\begin{array}{l}47.7 \text { Mtoe } \\
\text { (54\% imported) }\end{array}$ \\
\hline Oil Demand & 1980 & $\begin{array}{l}16 \text { Mtoe } \\
\text { (100\% imported) }\end{array}$ \\
\hline Electric Power Plant Capacity & 1980 & 29 GWe \\
\hline Electric Power Production & 1982 & $\begin{array}{l}96.3 \text { TWh-- } \\
27.7 \% \text { nuclear } \\
60.5 \% \text { hydro/geo. } \\
11.0 \% \text { oil } \\
0.8 \% \text { solid fuels } \\
96.5 \text { TWh-- } \\
38.7 \% \text { nuclear }\end{array}$ \\
\hline
\end{tabular}

NUCLEAR POWER

National Policy. Complete a total of 12 power stations by 1985-86, to provide $45 \%$ of Sweden's electricity; phase out al1 nuclear plants at the end of a 25-30 year operating life.

$\begin{array}{llll}\text { Nuclear Power Plant Capacity } & 1983 & 7.3 \text { GWe } \\ & 1985 & 8.4 \text { GWe } \\ & 1990 & 9.4 \text { GWe } & \\ & & & \\ & 1982 & \text { BWR: } & 7(1972-1981) \\ \text { Reactor Mix } & & & 2(1985) \\ & & \text { PWR: } & 2(1975,1981) \\ & & & 1(1983)\end{array}$

INDUSTRIAL FUEL CYCLE

Policy. Honor present commitments (870 tU) to foreign reprocessors, but put the rest of the spent fuel ( $6000 \mathrm{t}$ ) into a repository. No $\mathrm{Pu}$ recycle is planned. Waste management costs are paid from fees collected from the nuclear utilities. 
Waste Management Strategy. Store vitrified HLW (returned from reprocessors) and spent fuel for 30-40 years in an underground storage facility; encapsulate HLW glass in lead/titanium overpack, spent fuel in a copper canister; emplace in a deep geologic (crystalline rock) repository. New facilities: $3000 \mathrm{t}$ AFR; interim storage plants for conditioned reprocessing wastes; HLW and spent fuel encapsulation plants; repositories for reactor wastes, HLW glass, spent fuel, and other long-lived wastes.

$\begin{array}{rrr}\text { Cumulative Spent Fuel } & 1980 & 465 \mathrm{tU} \\ \text { Arisings (LWR) } & 1985 & 1,330 \mathrm{tU} \\ & 1990 & 2,360 \mathrm{tU} \\ & 2000 & 5,000 \mathrm{tU}\end{array}$

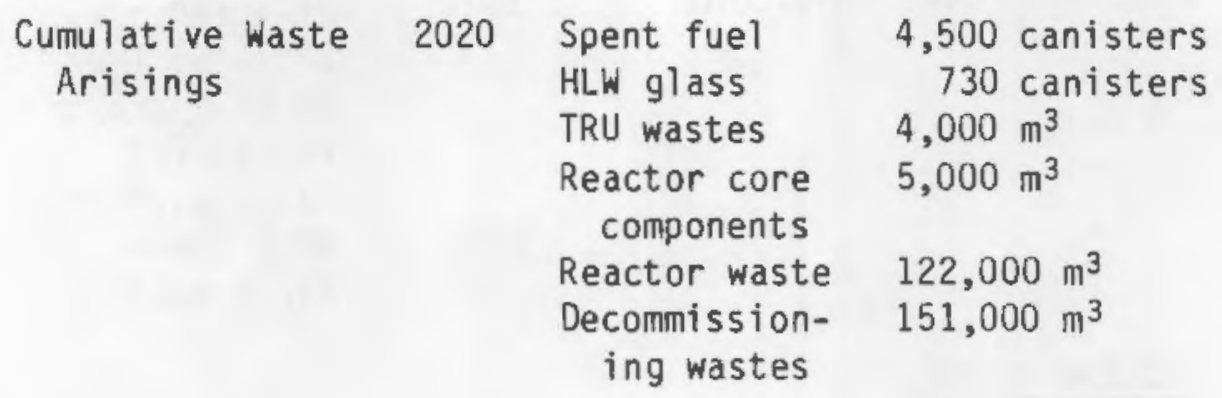

Industrial-Scale Activities

LWR fuel fabrication: $400 \mathrm{tU} / \mathrm{a}$.

Major Milestones

- CLAB AFR facility for spent fuels

- Repository for reactor wastes, SFR 1988

- HLW repository site selection 2000

- HLW repository operational 2020-2025

- Interim HLW dry storage facility 1990

- Encapsulated spent fuel repository 2026-2050

INTERNATIONAL RELATIONSHIPS

1. Membership in IAEA and OECD/NEA.

2. Waste management cooperative agreement with US.

3. Host country for Stripa project. 
ORGANIZATION

1. Waste Management

- National Board for Spent Fuel (NAK)--administers waste management fund collected from the nuclear utilities; oversees back-end-of-the-fuel-cycle activities

- Swedish Nuclear Fuel Supply Company (SKBF)-execute spent fuel and waste management program for the utilities; manage waste disposal R\&D programs.

2. Licensing Responsibilities

- Swedish Nuclear Power Inspectorate, SKI (erection and operation of nuclear facilities)

- Swedish National Institute of Radiation Protection

- National Swedish Franchise Board for Environment Protection

- Municipality where the facility is to be located (right of veto).

\section{ASEA-ATOM}

ASEA-Atom $A B$ (Headquarters)

P.0. Box 53

S-721 83 Västerås

Sweden

Te1: (46) 21100000

Telex: 40720

ASEA AB

High Pressure Laboratory

S-910 40 Robertsfors

Sweden

President

High Pressure Laboratory

Lars Halle Waste Treatment

H. T. Larker

Tore Waltersten 


\section{ASEA-ATOM (contd)}

Mission. Develop, design, and manufacture nuclear power plants and nuclear fuel components.

Sponsor. ASEA.

Waste Management R\&D. Application of hot isostatic pressing in spent fuel and waste treatment operations; AFR spent fuel storage facility design.

\section{CHALMERS UNIVERSITY OF TECHNOLOGY}

Chalmers Tekniska Högskola

Fack

S-402 20 Göteborg 5

Sweden

Nuclear Chemistry

Prof. Jan Rydberg

Dr. Bert Allard

R\&D. Radionuclide transport by groundwater, sorption on natural clays and rock minerals.

\section{KEMAKTA KONSULT AB}

Luntmahangatan 94

S-113 51 Stockholm

Sweden

Tel: (46) 8150380

Bertil Grundfelt.

Computer calculations on hydrology and nuclide migration. 
$\underline{\text { Sweden }}$

KTM (ROYAL INSTITUTE OF TECHNOLOGY)

KTM

S-100 44 Stockholm

Sweden

Department of Chemical Prof. Ivars Neretnicks

Engineering

Near- and far-field migration modeling, rock-matrix diffusion experiments.

NAK (NATIONAL BOARD FOR SPENT NUCLEAR FUEL)

Naenmden foer Hantering av Atvaent

Kaernbraensle

Kungsgatan 38

S-111 56 Stockholm

Sweden

Chairman

Sven Romanus

Director

Gerhard Rundquist

Chief Engineer

Ni Ts Rydell

Mission. Serve as the government's control agency to oversee back-end fuel cycle technology, financing and information.

Sponsor. Federal Government.

NATIONAL INSTITUTE OF RADIATION PROTECTION

National Institute of Radiation Protection

Fack

Box 60204

S-104 01 Stockholm 60

Sweden

Tel: (46) 8244080

Telex: 11771 SAFE RAD

Director

Department Director

Gunnar Bengtsson

Rad Waste Group

Dr. J. 0. Snihs

Ragnar Boge 
Sweden

\section{NUCLEAR SAFETY BOARD}

Radet for Kärnkräftsäkerhet

Nuclear Safety Board of the Swedish Utilities

Box 5864

S-102 48 Stockholm $\quad$ Tel: (46) 8679540

Sweden

Telex: 13108

Director

Thomas Eckered

Mission. Promote coordination and cooperation among the Swedish utilities in their nuclear power plant safety work.

Sponsors. The four Swedish nuclear power station owners.

SGU (GEOLOGICAL SURVEY OF SWEDEN)

Sveriges Geologiska Undersökning (SGU)

Vretgränd 18, Box 670

S-751 28 Uppsala

Sweden

Tel: (46) 18155280

Telex: 76154 GEOSWED $S$

Director-General

Gunnar Ekevärn

Hydrogeology

Geologic Waste Disposal

Leif Carlsson

Otto Brotzen

R\&D. Evaluation of rock formations for use as waste disposal sites (permeability; groundwater behavior, age and chemistry).

\section{STUOSVIK}

Studsvik Energiteknik $A B$

S-611 82 Nykoping

Sweden

Deputy Managing Director Nuclear Technology

Waste Management Department Waste Management Section
Te1: (46) 15580000

Telex: 64013 studs s

Stig Bergström

Lennart Devell

Àke Hultgren 


\section{STUDSVIK (contd)}

Mission. Nuclear energy R\&D to support Swedish power programs, contract research.

Sponsor. Federal government.

Fuel Cycle R\&D Programs. Waste isolation (field tracer migration tests in granite), HLW waste form characterization, LLW and ILW treatment, D\&D techniques, leaching of $\mathrm{UO}_{2}$ from spent fuel, biosphere migration, dosecalculations.

SWEDISH METEOROLOGICAL AND HYDROLOGICAL INSTITUTE

Swedish Meteorological and Hydrological

Institute

Folkborgsvägen 1

S-602 36 Norrköping

Sweden

Tel: (46) 11108000

SKBF (SWEDISH NUCLEAR FUEL SUPPLY COMPANY)

Svensk Kärnbränsleförsörjning $A B$ Box 5864

S-102 48 Stockholm

Tel: (46) 8679540

Sweden

Telex: 13108 SKBFS

President

Project Manager, CLAB Project

Manager, KBS Project

Chemistry

Geology and Hydrology

Material Sciences

Waste Forms

Engineered Barriers

Safety Analysis

Stripa Test Station

Foreign Activities and

Documentation

Repository for Reactor Waste

Sten Bjurstrom
Bo Gustafssten
Lars Bertil Nilsson
Fred Karlsson
Hans Carlşson/
Harald Ahagen
Lars Werme
Hans Forsström
Anders Bergstrom
Tönis Papp
Hans Carlsson/
Harald Ahagen
Claes Thegerström
Tommy Hedman


SKBF (SWEDISH NUCLEAR FUEL SUPPLY COMPANY) (contd)

Mission. Coordinate and arrange for nuclear fuel supply and reprocessing services for all Swedish nuclear power reactors; manage and fund $R \& D$ for the back end of the fuel cycle. Demonstrate that spent nuclear fuel and fuel reprocessing wastes can be disposed of safely and permanently.

Sponsors. State Power Board (50\%) and utilities

(Sydkraft, 25\% and Oskarshamn, 25\%).

Facilities 1. $\frac{\text { CLAB (Central Storage for Spent Fuel, to be located }}{\text { at Simpevarp) }}$

Mission. AFR storage facility. Design Capacity. Initially, 3000 t. Milestones. Start of construction, May 1, 1980; start of operation $(1500 \mathrm{t})$, January $1985 ; 1500$ additional, June 1985.

2. Stripa Mine

Stripa Mine Service $A B$

S-717 00 Stora

Sweden

Tel: (46) 58141420

Stripa Project Manager Hans Carlsson (SKBF/KBS)

Mine Operations Per-Axel Halen (Stallbergsbolagen)

Location. Near Kopparberg, $15 \mathrm{~km}$ north of Lindesborg and about $250 \mathrm{~km}$ west of Stockholm. Mission. Evaluate the use of granite formations as nuclear waste repositories, develop repository technology.

Description. Granite body, about $350-400 \mathrm{~m}$ below surface, adjacent to the Stripa iron mine. Sponsor. Project Sponsor: SKBF/KBS; Site Owner: Stallbergsbolagen. 


\section{SKI (SWEDISH NUCLEAR POWER INSPECTORATE)}

Statens Kärnkraftinspektion

Box 27106

S-102 52 Stockholm

Tel: (46) 8635560

Sweden

Telex: 11961 Sweatom S

Director

Waste Management

Lars Nordstrom

Alf Larsson

\section{SWEDISH STATE POWER BOARD}

Statens Vattensfallswerk

S-162 87 Vällingby

Tel: (46) 87395000

Sweden

Telex: 19653 Sutelvxs S

Thermal Engineering Development Per-Erik Åke Ahlstrom

Low- and Medium-Level Wastes Stig Pettarsson

Mission. Own and operate the power distribution grid in Sweden, produce power.

Sponsor. Swedish State (Ministry of Industry). 

SWITZERLAND 


$\begin{array}{ll}\text { *Asterisks } & \text { SWISS HOLIDAYS } \\ \text { January } 1 & \text { New Year's Day } \\ \text { *March/April } & \text { Good Friday } \\ \text { *March/April } & \text { Easter Monday } \\ \text { *May/June } & \text { Ascension Day } \\ \text { *ay/June } & \text { Whit Monday } \\ \text { August 1 } & \text { Day of Independence } \\ \text { December 25 } & \text { Christmas } \\ \text { December 26 } & \text { St. Stephen's Day }\end{array}$

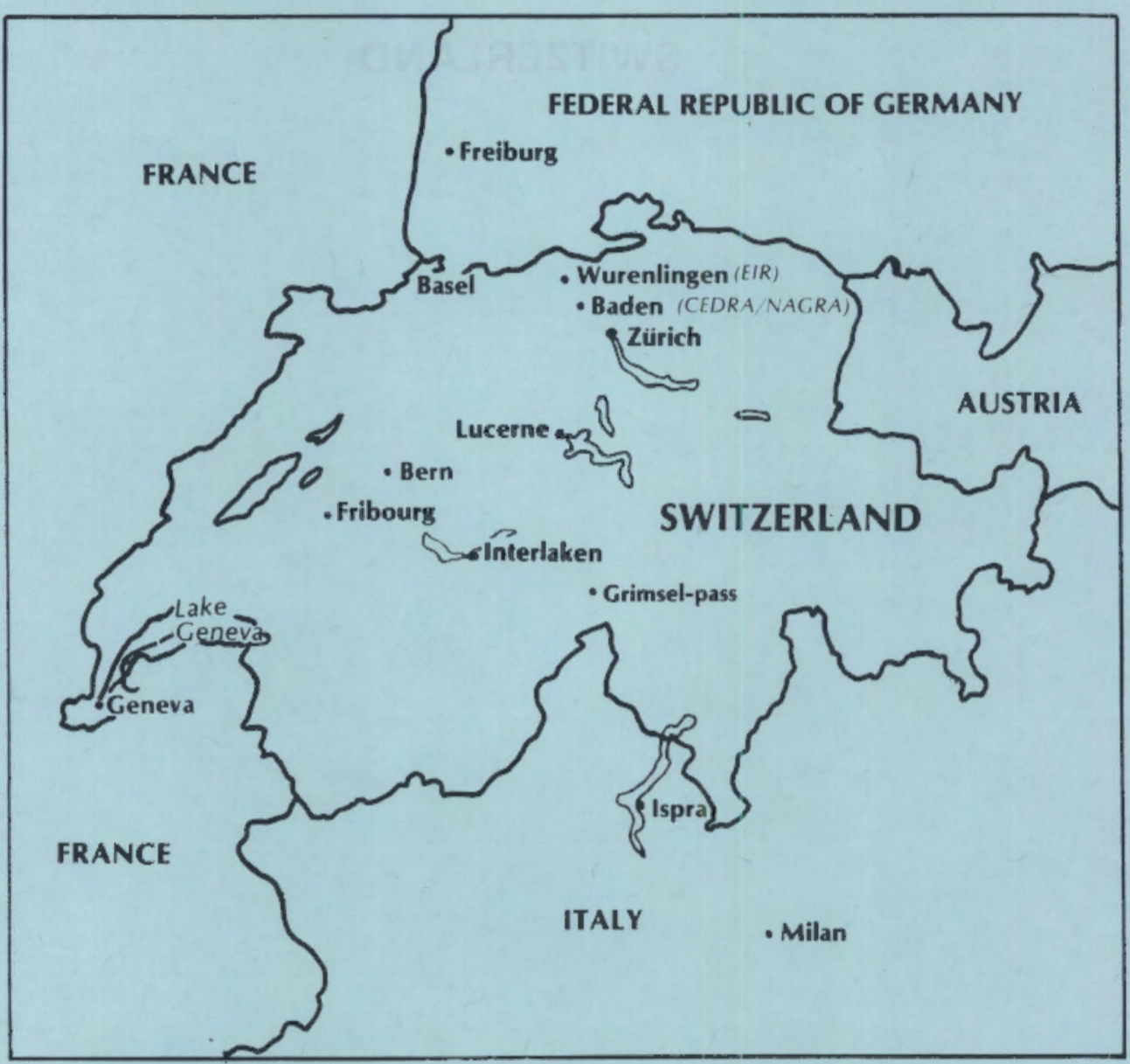




\section{SWITZERLAND}

ENERGY

Population

1982

6.4 million

Energy Demand

$1980 \quad 25.4$ Mtoe (57\% imported)

0i1 Demand

$1980 \quad 13.1$ Mtoe

(100\% imported)

Electric Power Plant Capacity 198015 GWe

Electric Power Production

198250.7 TWh--

$29 \%$ nuclear

$69 \%$ hydro/geo.

NUCLEAR POWER

National Policy. Government is pro-nuclear, but has encountered much public opposition. Policy now requires that by the end of 1985 the nuclear utilities establish a project guaranteeing safe waste management or lose their operating licenses.

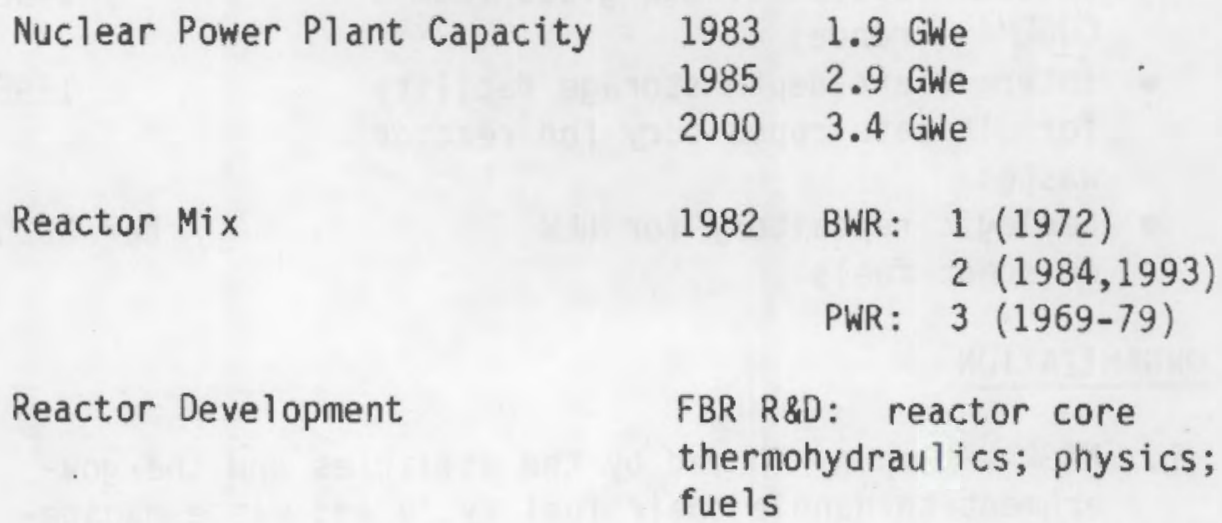

INDUSTRIAL FUEL CYCLE

Policy. Foreign reprocessing of spent fuels and $\mathrm{Pu}$ recycle to either LWRs or FBRs. 
Switzerland

Waste Management Strategy. Develop two waste repositories, one near the surface for LLW and a deep repository (crystalline rock) for HLW glass. Switzerland practiced sea-dumping of LLW until 1983.

$\begin{array}{crr}\text { Cumulative Spent Fuel } & 1980 & 380 \mathrm{tU} \\ \text { Arisings (LWR) } & 1985 & 650 \mathrm{tU} \\ & 1990 & 1,090 \mathrm{tU} \\ & 2000 & 2,000 \mathrm{tU}\end{array}$

Cumulative Waste Arisings: After 40 years' operation of their proposed nuclear power system:

$\begin{array}{lc}\text { LLW } & 100,000 \mathrm{~m}^{3} \\ \text { LLW/ ILW } & 70,000 \mathrm{~m}^{3} \\ \text { HLW glass } & 1,000 \mathrm{~m}^{3} \\ & \text { or } \\ \text { Spent fuel } & 15,000 \mathrm{~m}^{3}\end{array}$

Major Milestones

- Field laboratory in a granite formation

- Feasibility of safe and final 1984 disposal demonstrated

- Initial receipt of HLW glass from 1992 COGEMA (France)

- Intermediate-depth storage facility 1995 for ultimate repository for reactor wastes

- Geologic repository for HLW After 2020 or spent fuels

\section{ORGANIZATION}

1. NAGRA--company formed by the utilities and the government to handle their fuel cycle and waste management activities. Budget for 1980-1985 is about US $\$ 90$ million

2. Federal Institute for Reactor Research--nuclear R\&D

3. Federal Energy Office--sets criteria for waste management practices, including geologic disposal. 


\section{EIR (SWISS FEDERAL INSTITUTE FOR REACTOR RESEARCH)}

Eidgenठ̋ssisches Institut für

Reaktorforschung (EIR)

CH-5303 Würenlingen

Switzerland

Director

Deputy Director

Staff Assistant, Research

Materials Technology

Hot Laboratory

Metallurgy

Chemistry

Fuel Division (Waste Disposal

Concept, 1985)

Radioactive Waste Project

Fuel Program
Tel: (41) 56992111

Telex: 53714 eir ch

Prof.-Dr. H. Gränicher

M. Breu

Dr. U. Flứckiger

D. H. P. Alder

Dr. W. Hausmann

G. Ullrich

Or. P. Baertschi

H. Flury

Dr. G. Bart

R. W. Stratton

Mission. Nuclear research and development, including fuel cycle and waste management R\&D to support national programs.

Sponsor. Federal government--Department of Interior.

Waste Management R\&D. Incineration of TRU wastes, modeling of radionuclide migration through heterogeneous media, chemical behavior of radionuclides during migration, hydrological studies, sorption constants on different rocks, immobilization of LLW and ILW in cements, leaching rates on LLW and ILW waste forms, and long-term corrosion tests on waste package materials.

Facilities

ADA (Acid Digestion Plant for TRU Wastes)

Mission. Treat Pu-contaminated solids. Design Basis. Carbonization/digestion in $\mathrm{H}_{2} \mathrm{SO}_{4} / \mathrm{HNO}_{3}$ at $250^{\circ} \mathrm{C}$; capacity, $1 \mathrm{~kg} / \mathrm{hr}$ solid wastes. Milestones. Non-Pu runs, late 1981; Pu runs, 1982. 
Switzerland

\section{FEDERAL OFFICE OF ENERGY}

Federal Office of Energy

Nuclear Safety Department

$\mathrm{CH}-5303$ Würenlingen

Switzerland

Waste Management

Mission. License nuclear installations.
Tel: (41) 56982853

Telex: 59058

Or. U. Niederer

\section{FEDERAL OFFICE OF PUBLIC HEALTH}

Office Fédéral de la Santé Publique

Bollwerk 27

$\mathrm{CH}-3001$ Berne

Switzerland

Te1: (41) 31619620

Telex: 33880

Manager, Radioprotection Section Dr. H.-R. Stadelmann

NAGRA/CEDRA (NATIONAL COOPERATIVE FOR THE STORAGE OF RADIOACTIVE WASTE)

Nationale Genossenschaft fưr die Lagerung radioaktiver Abfalle (NAGRA)

or

Société Coopérative Nationale pour l'Entreposage de Déchets Radioactifs (CEDRA)

Parkstrasse 23

CH-5401 Baden

Switzerland

Tel: (41) 56205511

Telex: 57333

President

Dr. Rudolph Rometsch

Managing Director

Hans Issler

Geology

Nuclear Technology

Dr. Marc F. Thury

Dr. Charles McCombie

Mission. Provide for the safe disposal of radioactive wastes produced by the Swiss nuclear industry.

Sponsors. Swiss utilities and government. 
TAIWAN REPUBLIC OF CHINA 


\begin{tabular}{|c|c|}
\hline January 1-2 & Founding Day \\
\hline *January & Lunar New Year \\
\hline March 29 & Youth Day \\
\hline ^April & Tomb Sweeping Day \\
\hline $\begin{array}{l}\text { *June } \\
\text { September } 28\end{array}$ & $\begin{array}{l}\text { Dragon Boat Festival } \\
\text { Teachers' Day }\end{array}$ \\
\hline $\begin{array}{l}\text { September } 28 \\
\star 0 \text { ctober }\end{array}$ & $\begin{array}{l}\text { Teachers Day } \\
\text { Moon Festival }\end{array}$ \\
\hline October 10 & National Day \\
\hline October 25 & Taiwan Retrocession Day \\
\hline October 31 & $\begin{array}{l}\text { President Chiang Kai-Shek's } \\
\text { Birthday }\end{array}$ \\
\hline November 12 & Dr. Sun Yat-Sen's Birthday \\
\hline December 25 & Constitution Day \\
\hline
\end{tabular}

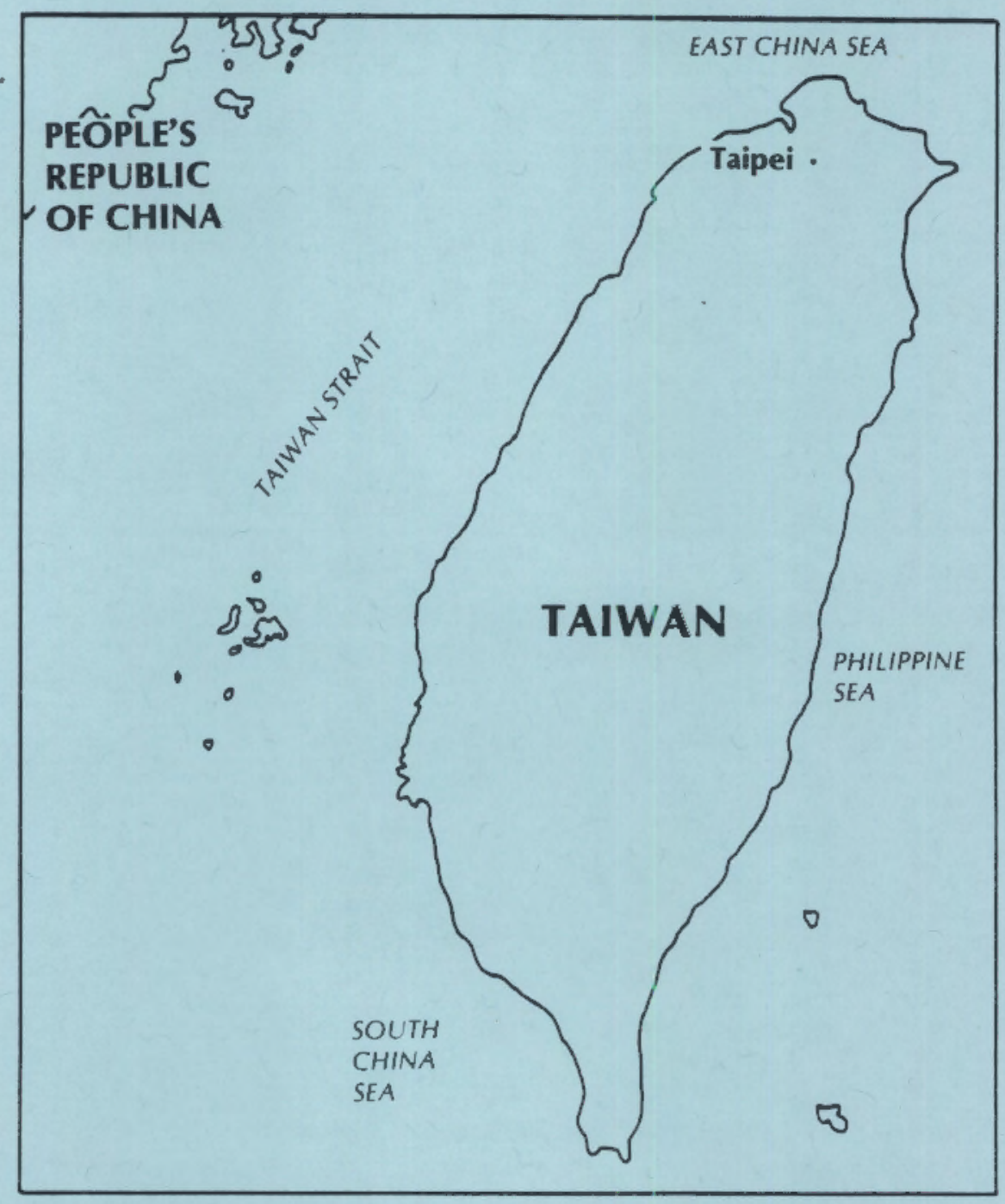




\section{TAIWAN}

\section{ENERGY}

\begin{tabular}{|c|c|c|}
\hline Population & 1982 & 18 million \\
\hline Energy Demand & 1980 & $79 \%$ imported \\
\hline Oil Demand & 1980 & $100 \%$ imported \\
\hline Electric Power Plant Capacity & 1991 & $\begin{array}{l}10.16 \text { GWe-- } \\
21.4 \% \text { nuclear } \\
18.17 \text { GWe-- } \\
29.9 \% \text { nuclear }\end{array}$ \\
\hline Electric Power Production & 1982 & $\begin{array}{l}40.9 \text { TWh-- } \\
30 \% \text { nuclear } \\
12 \% \text { hydro } \\
48 \% \text { oil } \\
10 \% \text { coal } \\
44.7 \text { TWh-- } \\
55 \% \text { nuclear }\end{array}$ \\
\hline
\end{tabular}

NUCLEAR POWER

National Policy. Look to nuclear power to meet rapidly growing demand for electric energy.

Nuclear Power Plant Capacity 1983

3.1 GWe

19854.0 GWe

$1990 \quad 4.9$ GWe

20008.7 GWe

Reactor Mix

1983 BWR: $4(1978-83)$

PWR: 2 (1984-85)

INDUSTRIAL FUEL CYCLE

Policy. Develop indigenous fuel production capability; fuel assembly (1986); fuel hardware; U0 2 pellets; $U_{6}$ conversion. May choose to reprocess.

Waste Management Strategy. Policy not yet defined on domestic reprocessing, HLW/spent fuel disposal; reactor wastes are to go to new National Waste Storage Facility on nearby Orchid Island. 


$\begin{array}{crr}\text { Cumulative Spent Fuel } & 1980 & 70 \mathrm{tU} \\ \text { Arisings (LWR) } & 1985 & 430 \mathrm{tU} \\ & 1990 & 1,140 \mathrm{tU} \\ & 2000 & 2,600 \mathrm{tU}\end{array}$

ORGANIZATION

- Nuclear power plants are owned by the government, operated by Taiwan Power

- Atomic Energy Council--regulatory functions; waste disposal

- Institute of Nuclear Energy Research--nuclear R\&D.

\section{AEC (ATOMIC ENERGY COUNCIL)}

Atomic Energy Council

67, Lane 144

Keelung Road, Section 4

Taipei, Taiwan

Republic of China

Tel: (56) $2392-4180$

Secretary General.

Telex: 26554

Director, Radwaste Administration

Dr. Victor Cheng

Director, Planning Division

Dr. Chao-Ming Tsai

$\mathrm{Mr}$. Yi-Ching Yang

Please Note: The customary form of address may be to use the surname first, followed by the given name. In case of uncertainty it is recommended to use both names. 


\section{INER (INSTITUTE OF NUCLEAR ENERGY RESEARCH)}

Institute of Nuclear Energy Research

P.0. Box 3

Lung tan Taiwan

Tel: (56) 2 381-4014

Republic of China

Telex: 34154 CAEC

Deputy Director

Dr. Jen-Chang Chou

Health Physics

(Ext. 2302)

Engineering Development and

W. L. Chen

Implement ation

Tise-Sheng Chou

(Ext. 2525)

Waste Management and Decon-

tamination

S. L. Hwang

Fuel Cycle R\&D. Solvent extraction technology; yellowcake conversion to $\mathrm{UO}_{2}$; production of $\mathrm{Zr}, \mathrm{TiO}_{2}$ and $\mathrm{TiCl}_{4}$; cement and thermoplastic waste forms for reactor wastes; HLW conditioning processes; irradiation of sewage sludge with spent fuels; burial of LLW.

\section{TAIPOWER (TAIWAN POWER COMPANY)}

Taiwan Power Company

242 Roosevelt Rd., Sec. 3

Taipei, Taiwan 107

Tel: (56) 2 396-7777

Republic of China

Telex: 2564 TPCAPD

Deputy Directors, Atomic Power

Mr. Eng Lin

(Ext. 2521)

Mr. R.C.T. Hsu

(Ext. 2522)

Please Note: The customary form of address may be to use the surname first, followed by the given name. In case of uncertainty it is recommended to use both names. 



\section{UNION OF SOVIET SOCIALIST REPUBLICS}




\section{SOVIET HOLIDAYS}

$\begin{array}{ll}\text { January } 1 & \text { New Year's Day } \\ \text { March } 8 & \text { Women's Day } \\ \text { May 1-2 } & \text { May Day } \\ \text { May } 9 & \text { Victory over Fascism } \\ \text { November 7-8 } & \text { October Revolution } \\ \text { December 5 } & \text { Constitution Day }\end{array}$

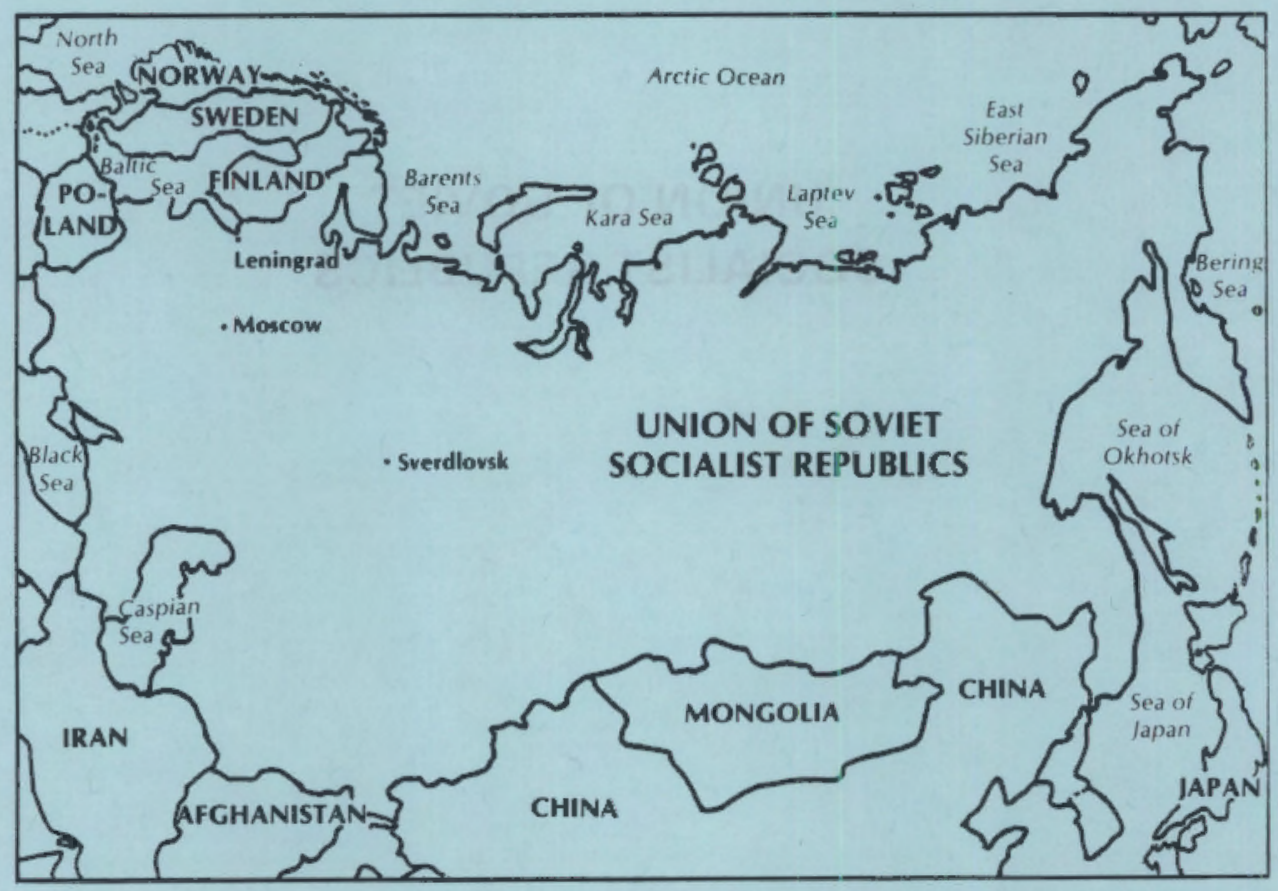




\section{ENERGY}

$\begin{array}{lcc}\text { Population } & 1980 & 265 \text { million } \\ \text { Electric Power Plant Capacity } & 1982 & 281 \text { GWe-- } \\ & & 6 \% \text { nuclear } \\ & 27 \% \text { oil } \\ & & 27 \% \text { gas } \\ & 25 \% \text { coal } \\ & & 15 \% \text { hydro } \\ & & 20-22 \% \text { nuclear } \\ & 1993 \quad 460 \text { GWe } \\ & & \\ \text { Electric Power Production } & 1982 & 1277 \text { TWh-- } \\ & & 6.5 \% \text { nuclear } \\ & 1985 & 1550 \text { TWh-- } \\ & & 14 \% \text { nuclear }\end{array}$

NUCLEAR POWER

National Policy. Major program to develop nuclear power, to avoid transport of fossil fuels from east of the Ural Mountains to European Russia.

Nuclear Power Plant Capacity 1982 198525 GWe

1992 90-100 GWe

Reactor Mix

1982 LGR: $18(1958-82)$

1 (1984)

PWR : $12(1964-82)$

$3(1983-85)$

LMFBR: $2(1973,1980)$

Reactor Development LMFBRs, 1,500-MWe PWRs

INDUSTRIAL FUEL CYCLE

Policy. Complete domestic fuel cycle capability, including enrichment, fuel fabrication $\left(\mathrm{UO}_{2}\right.$ and $\left.\mathrm{MOX}\right)$; develop commercial reprocessing capability.

Waste Management Strategy. Vitrify HLW, provide terminal storage in geologic repository. 
ORGANIZATION

Nuclear Program Control

- USSR State Committee on Utilization of Atomic Energy

- Ministry of Energy and Electrification.

$\underline{R \& D}$

- Institute of Physical Chemistry, Moscow, a branch of the USSR Academy of Sciences (geologic waste disposal; waste form properties; personnel:

V. I. Spitsyn,(a) N. E. Brezneva, M. K. Pimenov)

- V. G. Khlopin Radium Institute, Leningrad (chemical separation; fuels reprocessing; geochemistry)

- All-Union Scientific Research Institute for Inorganic Materials, Moscow (properties of solid waste forms; personnel: A, $\{$. Polyakov, (a) V. V. Kulichenko, (a) N. V. Krylova $(a)$ )

- Chemical Plant Research Institute, Sverdlovsk (vitrification pilot plants).

\section{STATE COMMITTEE ON THE UTILIZATION}

OF ATOMIC ENERGY

State Committee for Atomic Energy

Staromonetny Pereulok 26

Moscow 109180

USSR

Chai rman

A. M. Petrosyants

Deputy Chairman

Prof. I.D. Morokhov

Waste Management R\&D. USSR laboratories are developing processes for treating spent fuel (cladding, thermal decladding, meltdown of hulls), improved partitioning of TRU wastes, handling off-gases, and storing $85 \mathrm{~K} r$.

(a) Also a member of the State Committee on the Utilization of Atomic Energy

UR-2 
Facilities $^{(a)}$

1. Reprocessing Pilot Plant (radioactive)

Owner. Khlopin Radium Institute, Leningrad. Mission. Develop LWR fuel reprocessing technology. Design Basis. Chop-leach head-end; Purex flowsheet; capacity, $3 \mathrm{~kg} /$ day uranium. History. Startup, 1973.

2. Cold Pilot Plant-Vitrification

Mission. Develop waste vitrification technology. Design Basis. Liquid-fed ceramic melter, two-chamber unit; $150 \mathrm{l} / \mathrm{hr}$ HLLW; $30 \mathrm{l} / \mathrm{hr}$ glass; product, phosphate glass blocks. History. Startup, 1974.

3. KS-KT-100 (cold pilot plant-vitrification)

Location. Chemical Plant Research Institute, Sverdlovsk. Mission. Develop vitrification technology for high-AT, high-Na HLw. Design Basis. Fluid bed calciner; in-crucible melter (two-stage process); capacity, $20 \mathrm{~kg} / \mathrm{hr}$ glass; 160-180 kg glass/batch; product, phosphate glass blocks. Personnel. V. I. Davydov; v. v. Dolgov.

\section{KHLOPIN RADIUM INSTITUTE}

Khlopin Radium Institute

U1. Rentgena 1

Leningrad 22

USSR

Chief of Laboratory

Yergeniy Shashukov

(a) Because there is only limited information available, it is not always known at which nuclear agency a facility is located. 



\section{UNITED KINGDOM}




\begin{tabular}{|c|c|}
\hline \multicolumn{2}{|c|}{ BRITISH HOLIDAYS } \\
\hline *Asterisks & movable holidays \\
\hline January 1 & New Year's Day \\
\hline \# March/April & Good Friday \\
\hline \#arch/April & Easter Monday \\
\hline May 3 & May Day \\
\hline *May & $\begin{array}{l}\text { Whitsuntide or Spring } \\
\text { Bank Holiday }\end{array}$ \\
\hline August 30 & Summer Bank Holiday \\
\hline December 25 & Christmas \\
\hline December 26 & Boxing Day \\
\hline
\end{tabular}

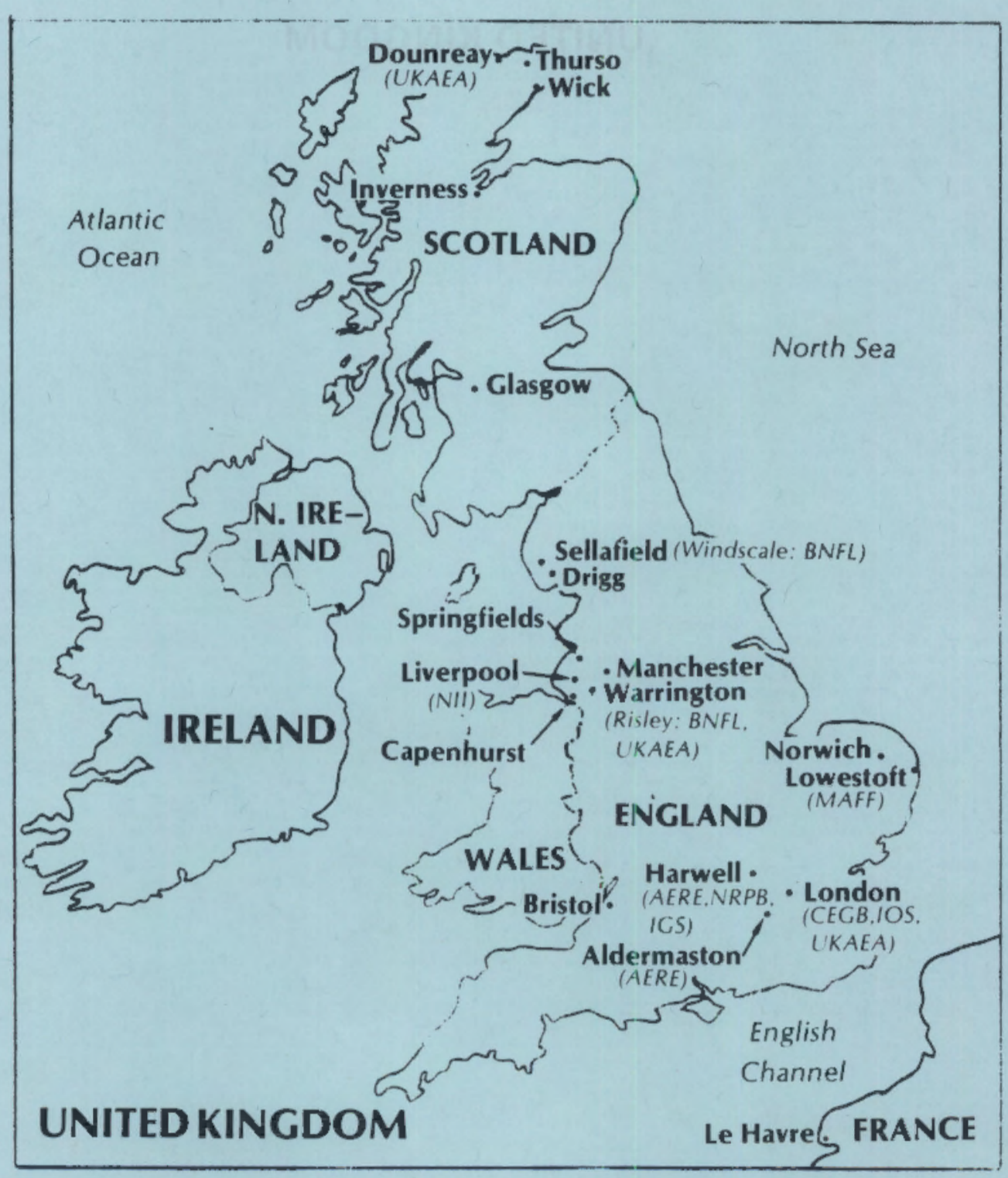




\section{UNITED KINGDOM}

ENERGY

Population

Energy Demand

Dil Demand

Electric Power Plant Capacity

Electric Power Production
198260 million

1980201.5 Mtoe

198081 Mtoe

(1\% imported)

198082 GWe

1980285 TWh--

$13 \%$ nuclear

$73 \%$ solid fuels

$12 \%$ oil \& gas

$2 \%$ hydro

1982254 TWh--

$16.4 \%$ nuclear

NUCLEAR POWER

National Policy. Aggressive development of nuclear power, based to date on gas-cooled reactors but, diversifying to PWRs and eventually to FBRs.

Nuclear Power Plant Capacity
1983
6.4 GWe
1985
10.0 GWe
200013.6 GWe
1982 GCR: $26(1956-72)$
AGR: $5(1976-82)$
$9(1983-88)$
LMFBR: 1 (1976)
Major LMFBR development program, including fuel cycle

Reactor Mix

Reactor Development

INDUSTRIAL FUEL CYCLE

Policy. Reprocess and recycle Pu to FBR system; develop and maintain complete fuel cycle capability (enrichment, $\mathrm{UO}_{2}$ and MOX fuel fabrication, spent fuel reprocessing); sell fuel cycle services to foreigners. 
Waste Management Strategy. Reprocess spent fuels as rapidly as plant capacity permits; vitrify HLW (French technology); long-term interim storage of HLW glass, deferring decision on disposal; shallow-land burial or sea-dump of LLW and ILW.

Cumulative Spent Fuel

Arisings (LWR and AGR)

$\begin{array}{rr}1980 & 250 \mathrm{tU} \\ 1985 & 830 \mathrm{tU} \\ 1990 & 2,000 \mathrm{tU} \\ 2000 & 5,330 \mathrm{tU}\end{array}$

Industrial-Scale Activities

1. Power production (GWe): 1981--9.8; 1990--14.8.

2. Uranium conversion (Springfields)

- UF 6 production--9500 t/a

- UF ${ }_{6}-\mathrm{UO}_{2}$ conversion--700 t/a.

3. Uranium enrichment (Capenhurst)

- gas diffusion plant--400 tSWU/a

- centrifuge plant--225 tSWU/a.

4. Fuel fabrication

- U metal (Magnox)--2500 t/a (Springfields); $\mathrm{UO}_{2}$ fuels $--500 \mathrm{t} / \mathrm{a}$ (Springfields)

- MOX fuels for recycle to FBRs (BNFL/Sellafield): current--10 t/a; future new capacity--20 t/a, 1984 and $50 \mathrm{t} / \mathrm{a}, 1987$.

5. Fuel reprocessing

- Magnox fuels (Sellafield)--5 to $7 \mathrm{t} / \mathrm{a}$

- $\mathrm{UO}_{2}$ fuels (THORP) $--6 t /$ day (1990)

- FBR fuels (PFR reprocessing pilot plant, Dounreay) $--50 \mathrm{~kg} \mathrm{HM} /$ day

- FBR fuels (CDFR reprocessing plant, 1993).

6. HLW vitrification. Design and construction of Windscale Vitrification Plant (1988).

7. Waste disposal. Underground repository for conditioned ILW, 1987-88. 


\section{INTERNATIONAL RELATIONSHIPS}

1. Member of European Communities, IAEA and OECD/NEA.

2. LMFBR exchange agreement with US DOE.

3. BNFL has partnership in URENCO (uranium enrichment), United Reprocessors GmbH (reprocessing), and Nuclear Transport Limited (spent fuel transport) and Pacific Nuclear Transport Limited.

\section{ORGANIZATION}

1. UKAEA--nuclear research; laboratories at Harwell, Risley, Springfields, Dounreay

2. Department of Envi ronment--develops waste management strategy and coordinates waste management R\&D

3. BNFL--commercial fuel cycle for domestic and foreign customers

4. NIREX--disposal of LLW and ILW

5. Institutes of Geological and Oceanographic Sciences-supporting R\&D for the waste management program

6. NRPB--environmental R\&D

7. Nuclear Installations Inspectorate--licensing. 


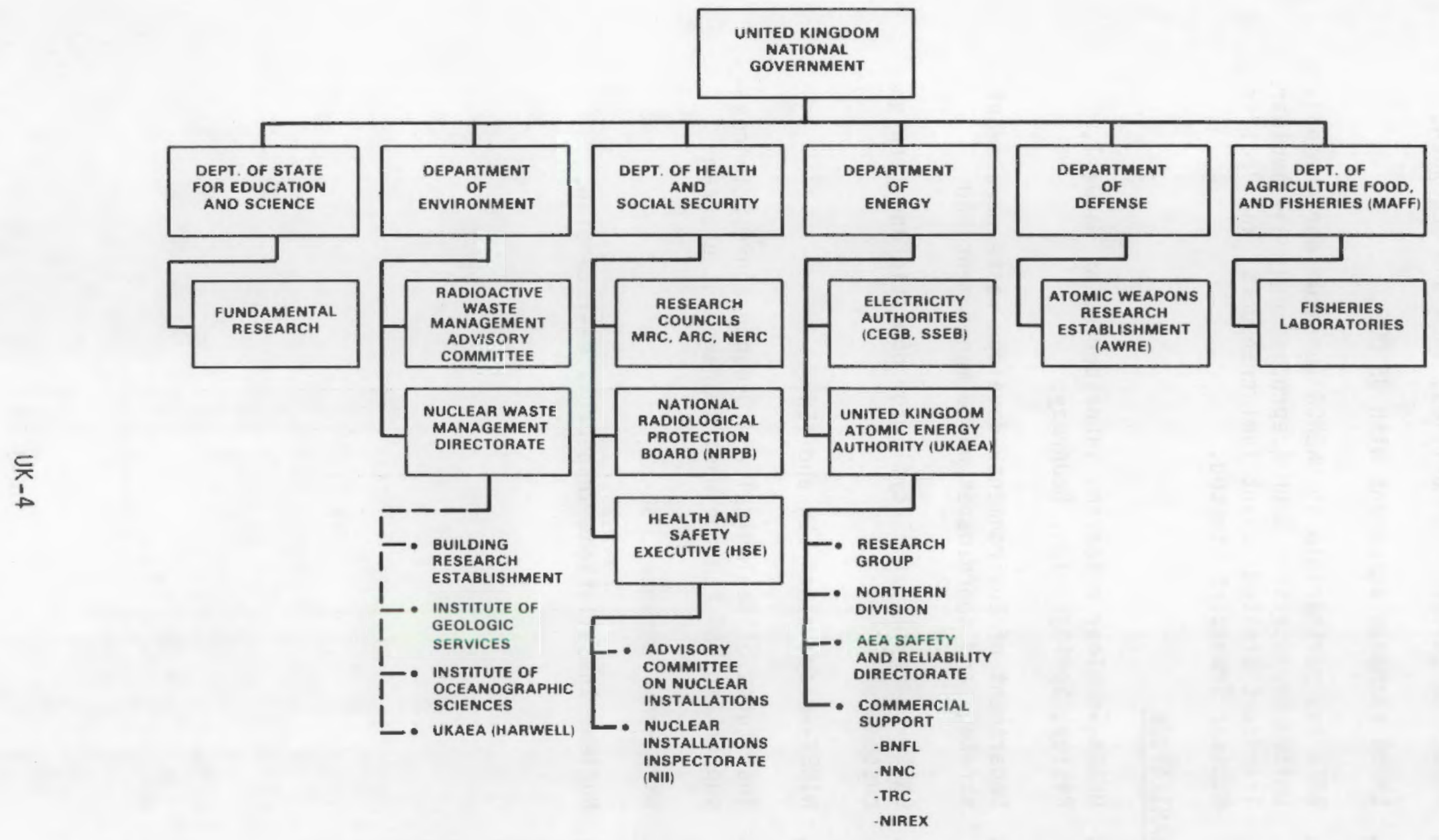

FIGURE UK-1. Nuclear Fuel Cycle Responsibilities 


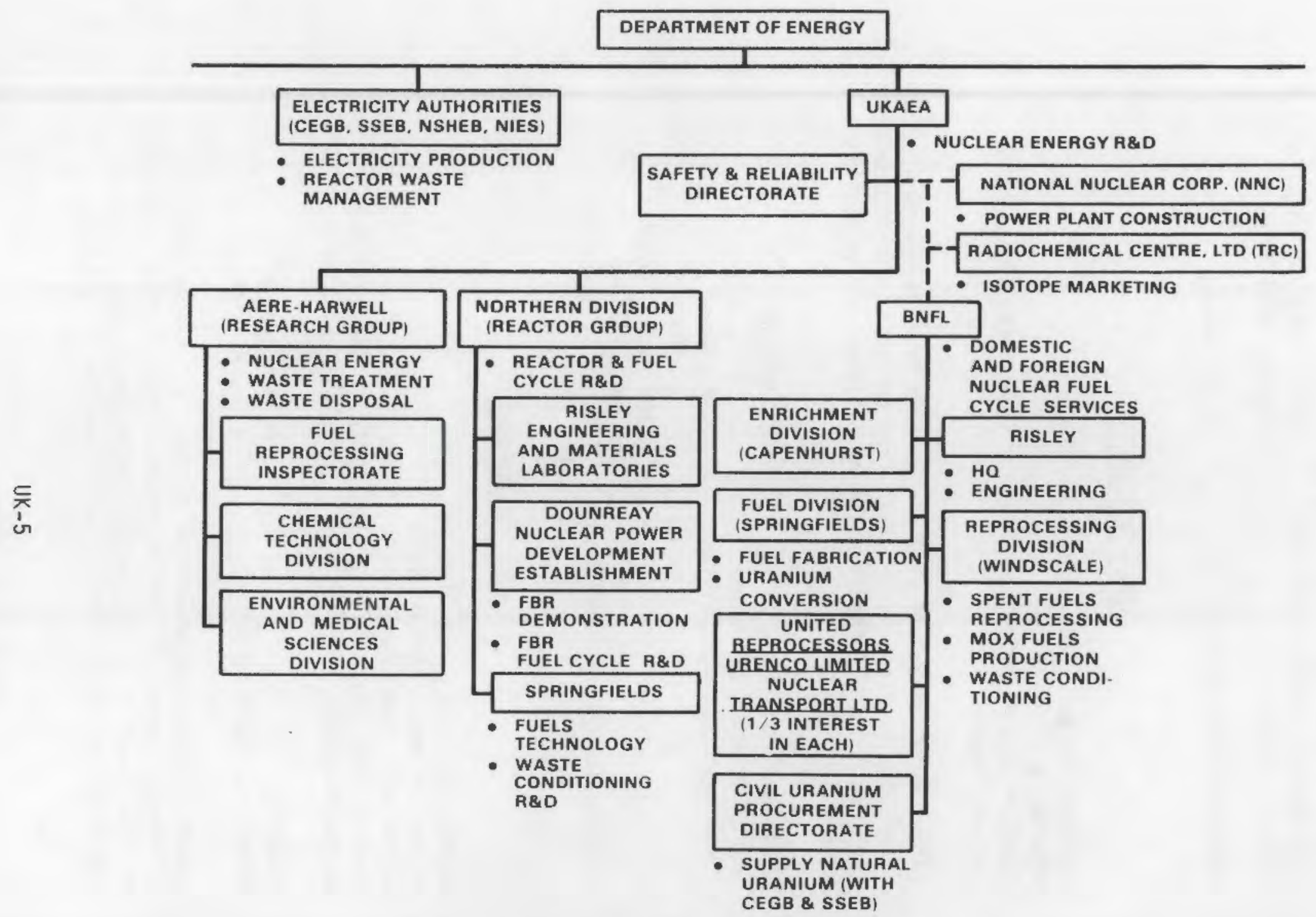

FIGURE UK-2. Nuclear Fuel Cycle Responsibilities Department of Energy 
United Kingdom

\section{AWRE (ALDERMASTON)}

Atomic Weapons Research Establishment

Aldermaston, Reading RG7 4PR

Te1: (44) 73564111

United Kingdom

Telex: $848104 / 5$

Manager, Waste Management

E. Whitehead

BNFL: RISLEY (HQ)

British Nuclear Fuels Limited

Risley, Warrington

Cheshire WA3 6AS

Tel: (44) 92535953

United Kingdom

Telex: 627581

[About 20 miles by official car or taxi from Manchester International Airport; or train from London to Warrington (approx. 3 hours), then 6 miles by official car or taxi to Risley.]
Chairman, BNFL
C. Allday
Deputy Managing Director
Dr. D. G. Avery
R\&D Reprocessing Division
N. R. Geary
L. P. Shortis
Alan D. W. Corbet
D. W. Clelland
Waste Management Development
Dr. K. R. Schneider

\section{BNFL: SELLAFIELD}

British Nuclear Fuels Limited

Windscale and Calder Works

Sellafield, Seascale

Cumbria CA20 1PG

United Kingdom

Tel: (44) 94028333

Telex: $64237 / 8$

Director

Gordon Steele

Director, Reprocessing

W. L. Wilkinsen

[By train from London-Euston Station to Carlisle Station (4 hours); transport can be arranged by BNFL from Carlisle to site (approx. 1-1/2 hours). From Manchester International Airport to site by car is approx. 3 hours.] 
BNFL: SELLAFIELD (contd)

\section{Reprocessing Facilities}

1. B204-Windscale

Mission. Reprocess military fuels.

Design Basis. BUTEX process; "no-maintenance" concept.

History. Dn-line, 1952-1964. Converted to oxide fuel head-end plant for B205-Windscale, 1969.

2. B205-Windscale

Mission. Reprocess Magnox (magnesium-clad, U metal) fuels from UK GCRs. Oxide head-end added in 1969 to handle AGR and LWR fuels.

Design Basis. Magnox fuels: mechanical declad;

PUREX flowsheet; "no-maintenance" concept; nominal capacity, $8 \mathrm{tHM} /$ day. New oxide head-end: shearleach and one BUTEX cycle in B204; solution transferred to B205 for further processing; remote maintenance; nominal capacity, $1.3 \mathrm{tHM} /$ day. Liquid HLW storage: SS tanks, $70 \mathrm{~m}^{3}$ and $150 \mathrm{~m}^{3}$, in SS-lined concrete cells. History. Magnox fuels: B205 startup, 1967; annual throughput of Magnox fuels, 1000-1200 thM. Oxide head-end operation, 1969-1973; 90 t oxide fuel processed before the plant was shut down after a contamination release incident.

3. Windscale Reprocessing Test Unit (nonradioactive)

Mission. Test the THORP first solvent extraction cycle.

Design Basis. Purex flowsheet; nominal capacity, $4 \mathrm{t} / \mathrm{a}$ LWR fuels. History. Startup, 1979. 


\section{BNFL: SELLAFIELD (contd)}

4. THORP (Thermal Dxide Reprocessing Plant)

Mission. Reprocess AGR and domestic or foreign LWR fuels.

Design Basis. Purex flowsheet, pulsed columns and mixer-settlers. AVM process for HLW vitrification. Nominal capacity, 6 tU/day.

Milestone. Startup, 1989 or later.

5. Windscale Reprocessing Pilot Plant (radioactive)

Mission. Support THORP design and operation. Design Basis. 1/5000 scale.

MoX Fuel Fabrication Facilities

1. MoX Fuel Fabrication Plant

Design Capacity. $10 \mathrm{t} / \mathrm{a}$ FBR fuels.

2. MoX Fuel Fabrication Plant

Design Basis. Gel precipitation; capacity, $0.5 \mathrm{~kg} /$ day. Milestone. Startup, 1981-82.

3. MOX Fuel Fabrication Production Plants

Design Basis. Mechanical mixing or coprecipitation. $20 \mathrm{t} / \mathrm{a}$ (startup, 1984); $50 \mathrm{t} / \mathrm{a}$ (startup, 1987).

\section{Waste Treatment Facilities}

1. FSIF (cold demonstration plant-vitrification)

Mission. Test prototype equipment and maintenance techniques. Design Basis. French AVM process (rotating-tube calciner/metal melter); capacity, $11 \mathrm{~kg} / \mathrm{hr}$ glass; product, borosilicate glass blocks. Milestone. Startup, 1982. 


\section{BNFL: SELLAFIELD (contd)}

2. Windscale Vitrification Plant

Mission. Solidify Windscale HLW.

Design Basis. AVM process; product, borosilicate glass blocks. Milestone. Startup, 1987.

3. Incinerator Pilot Plant (radioactive)

Mission. Volume reduction of contaminated waste. Design Basis. Pyrolysis-incineration process; capacity, $5 \mathrm{~kg} / \mathrm{hr}$. History. Startup, 1973.

4. Windscale Waste Treatment Complex

Mission. Prepare non-HLW for ocean dumping; extract Pu from process residues. Construction. Ten-year period, starting in 1981 (estimated cost: $\left.\$ 225 \times 10^{6}\right)$.

\section{BNFL: SPRINGFIELDS}

British Nuclear Fuels Limited

Springfields Works

Salwick, Preston, Lancashire PR4 OXJ

United Kingdom

Tel: (44) 772728262

Chief Technical Manager

Telex: $\quad 67526 / 7$

A. Naylor 
United Kingdom

BRE (BUILDING RESEARCH ESTABLISHMENT--DOE)

Building Research Establishment

(Department of the Environment)

Building Research Station

Garston, Watford WD2 7JR

Tel: (44) 927374040

United Kingdom

Telex: 923220

Assistant Director, Geotechnics

Dr. J. B. Menzies

and Structural Engineering

Head, Geotechnics Division

Seabed Disposal

J. B. Boden

Continental Disposal

T. Freeman

Ms. C. M. Cooling

Waste Management Mission. Emplacement engineering and related activities; rock mechanics R\&D.

\section{CEGB (CENTRAL ELECTRICITY GENERATING BOARD)}

Central Electricity Generating Board

Sudbury House

15 Newgate Street

London, EC1A 7AU

United Kingdom

Tel: (44) 1 248-1202

Telex: 883141

Chairman

Director, Plant Engineering

Sir W. Marshall

Director, Research

P. M. Billam

Fuel and Core Division

Or. J. K. Wright

Dr. B. C. Masters

DOE (DEPARTMENT OF THE ENVIRONMENT)

Department of the Environment

Nuclear Waste Management Division

43 Marsham Street

London SWI 3py

Tel: (44) $1212-6315$

United Kingdom

Telex: 22221 
DOE (DEPARTMENT OF THE ENVIRONMENT) (contd)

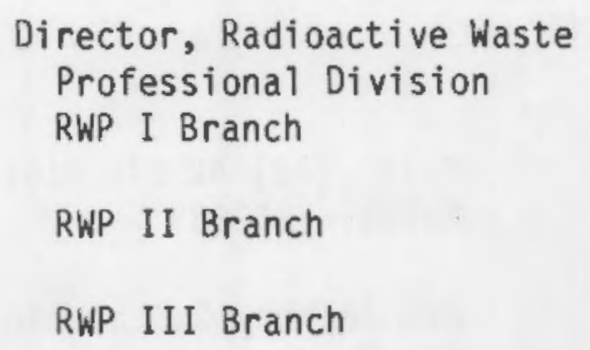

Dr. Frank S. Feates

(44) 1 212-8804

Brian Hookway

(49) 1 212-6937

Dr. Allan G. Duncan

(49) $1212-8673$

Dr. Haydn Richards

(49) 1 212-6937

Waste Management Responsibility. Administer UK waste management programs; fund and coordinate waste treatment and waste isolation R\&D at Harwell, IGS, NRPB, etc.; regulate discharge of radioactive materials to the environment.

\section{$\underline{\text { IGS (INSTITUTE OF GEOLOGICAL SCIENCES) }}$}

Institute of Geological Sciences (HQ) Keyworth

Nottingham, NG12 5GG

United Kingdom

Tel: (44) 60776111

Telex: 378173 ISGKEY G

Director

Fluid Processes

Dr. G. M. Brown

Dr. John D. Mather

Institute of Geological Sciences

Harwell Laboratory

Building 151

Harwe11, 0xon 0x11 ORA

United Kingdom

Tel: (49) 23524141

Telex: 83135 ATOMHA G

Institute of Geological Sciences

Exhibition Road

London, SW7 2DE

Tel: (44) 01 589-3444

Telex: 8812180 GEOSCI G

Assistant Director, Geophysics David A. Gray and Hydrogeology 
United Kingdom

\title{
IOS (INSTITUTE OF OCEANOGRAPHIC SCIENCES)
}

\author{
Institute of Oceanographic Sciences \\ Brook Road \\ Wormley, Godalming \\ Surrey GU8 5UB \\ United Kingdom \\ Te1: (49) 428794141 \\ Telex: 858833 \\ Director \\ Nuclear Waste \\ Dr. Anthony S. Laughton \\ Dr. T. J. G. Francis

\section{MAFF FISHERIES LABORATORIES} \\ Fisheries Radiobiological Laboratories \\ Ministry of Agriculture, Fisheries, and Food \\ Pakefield Road \\ Lowestoft, Suffolk NR32 OHT \\ Tel: (49) 5024381 \\ United Kingdom \\ Telex: 97470 \\ Director, Fisheries Research \\ Alan Preston \\ Directorate \\ Head, Aquatic Environment Harry Hill \\ Protection Division $\quad$ (49) 50262244 \\ NRPB (NATIONAL RADIOLOGICAL PROTECTION BOARD) \\ National Radiological Protection Board \\ Chilton Didcot \\ Oxfordshire OX11 ORQ Tel: (49) 235831600 \\ United Kingdom Telex: 837124 \\ Director \\ Secretary \\ H. J. Dunster \\ Assistant Director, Nuclear \\ Dr. R. H. Clarke \\ Assessments \\ Nuclear Assessments \\ G. A. M. Webb \\ Waste Management \\ Ms. M. D. Hill \\ Environmental Transfer \\ Dr. M. J. Clark \\ Dr. G. S. Linsley
}


NIREX (NUCLEAR INDUSTRY RADIOACTIVE WASTE EXECUTIVE)

Nuclear Industry Radioactive Waste Executive

Harwell

Didcot, 0xon 0x11 ORA

United Kingdom

Tel: (49) 23524141

Managing Director

Repository Development

M. E. Ginniff

Disposal at Sea; International

H. Beale

Relations; Packaging and

Transport

Waste Inventory R\&D; Packaging

and Transport

Dr. J. Grover

Mission. Locate, develop and operate facilities and sites for disposal of LLW and ILW.

Sponsors. UKAEA, BNFL, regional electricity generating boards.

\section{NII (NUCLEAR INSTALLATIONS INSPECTORATE)}

Nuclear Installations Inspectorate

Thames House North

Millbank, London SW1P 4QJ Tel: (44) 1 211-3000

United Kingdom

Telex: 918777 ENERGY G

Nuclear Installations

R. Anthony

Overseas Liaison

J. S. MacLeod

RWMAC (RADIOACTIVE WASTE MANAGEMENT ADVISORY COMMITTEE)

Mission. Advise Secretaries of State for the Environment, Scotland and Wales, on waste management.

Organization. Eleven independent scientists and representatives from four nuclear and electric utilities and three labor unions form the committee that reports progress to Parliament every year. 
RWMAC (RADIOACTIVE WASTE MANAGEMENT ADVISORY COMMITTEE) (contd)

Chairman (also Vice-Chancellor, Sir Denys Wilkinson University of Sussex, Brighton, United Kingdom)

\section{SRD (SAFETY AND RELIABILITY DIRECTORATE)}

UKAEA Safety and Reliability Directorate

Wigshaw Lane, Culcheth

Warrington WA3 6AT

Tel: (44) 92531244

United Kingdom

Telex: 629301 atonry-g

Mission. Advise the UKAEA on safety, coordinate reactor safety research, and provide advice and services on safety to government and industry.

\section{UKAEA (UNITED KINGDOM ATOMIC ENERGY AUTHORITY)}

United Kingdom Atomic Energy Authority

11 Charles II Street

London SWI 4QP

United Kingdom

Tel: (44) 19305454

Telex: 22565

Chairman

Sir Peter Hirsch

\section{UKAEA: HARWELL}

UKAEA Atomic Energy Research Establishment

AERE, Harwell

Didcot, 0xon Ox11 ORA Tel: (44) 23524141

United Kingdom

Telex: 83135 
UKAEA: HARWELL (contd)

$\begin{array}{ll}\begin{array}{l}\text { Director (and Member of UK } \\ \text { Radioactive Waste Management }\end{array} & \text { Dr. L. E. J. Roberts } \\ \text { Advisory Council, RWMAC) } & \\ \begin{array}{ll}\text { Authority's Fuel Processing } \\ \text { Director }\end{array} & \text { Dr. R. H. Flowers } \\ \text { Authority's Underlying Research } & \text { Dr. J. Williams } \\ \text { Director and Harwell Research } & \\ \text { Director, Nuclear Research } & \\ \begin{array}{l}\text { Chemical Technology } \\ \text { Radioactive Waste }\end{array} & \text { Dr. R. L. Nelson } \\ \text { Management } & \text { M. J. Smyth } \\ \text { Fuel Cycle } & \text { Dr. J. R. Findlay } \\ \text { Reactor Chemistry } & \text { R. L. Faircloth } \\ \text { Industrial Chemistry } & \text { Dr. K. W. Carley- } \\ \text { Liquid Processes } & \text { Macauly } \\ \text { Technology } & \text { B. Partridge } \\ \text { Materials Processing } & \text { M. J. S. Smith }\end{array}$

Waste Management Facilities

1. HARVEST Pilot Plant (nonradioactive)

Mission. Develop waste vitrification technology. Design Basis. Rising-level pot calcination/ vitrification process; capacity, $240 \mathrm{~kg}$ glass/run; product, borosilicate glass blocks. History. Startup, 1975.

2. Harwell Ceramic Melter Test Unit (nonradioactive)

Mission. Develop ceramic melter capability for Design Basis. Liquid-fed ceramic melter; capacity, $700 \mathrm{~kg} /$ day glass; product, borosilicate glass. Milestone. Startup, Fall 1981-Spring 1982. 


\title{
UKAEA: RISLEY
}

\author{
Risley Nuclear Power Development Establishment \\ UKAEA (Northern Division) \\ Risley, Warrington \\ Cheshire WA3 6AT \\ United Kingdom \\ Tel: (44) 92531244 \\ Telex: 629301
Director, Process Technology and Safety Waste Management (General)
Decommissioning \\ R. H. Allardice \\ Ext. 2181 \\ H. Barton \\ Ext. 2451 \\ D. F. Rawson
}

UKAEA: DOUNREAY

Dounreay Nuclear Power Development

Establishment

UKAEA (Northern Division)

Thurso, Caithness KW14 7TZ

Scotland

Tel: (44) 84762121

United Kingdom

Telex: 75297

[From London by air to Wick (via Aberdeen), then approx. 30 miles by car to Dounreay; or by train from London to Thurso (via Inverness), then approx. 10 mi les by car to Dounreay.]
Director
Clifford W. Blumfield
Assistant Director, Fuels
0 . Pugh

\section{Reprocessing Facilities}

PFR Reprocessing Plant

Mission. Reprocess Dounreay Prototype Fast Reactor (MOX) fuels.

Design Basis. Shear single pins and leach; PUREX process; capacity $9-10 \mathrm{tHM} / \mathrm{a}$ of 180 -day cooled PFR assemblies with $8-10 \%$ burnup.

History. Dounreay Fast Reactor fuels processed from 1961 to 1975; plant rebuilt to handle PFR oxide fuels, resumed operation in October 1980. 
United Kingdom

\section{UKAEA: SPRINGFIELDS}

UKAEA Springfields Nuclear Power Development Laboratories

Salwick, Preston

Lancashire PR4 ORR

United Kingdom

Te1: (44) 772728262

Telex: 67545

Chemical Engineering Division

Chemical Plant Development

Process Engineering Technology

Head-End and Solid Waste

Management

John V. Shennan

P. Laurie Allen

Cliff Etherington

Brian E. Meredith 
0

○

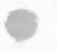

.

. 


\section{INTERNATIONAL AGENCIES}


CMEA (COUNCIL FOR MUTUAL ECONOMIC ASSISTANCE)

\section{MEMBER STATES}

Bulgaria

Mongolia

Cuba

Poland

Czechos lovakia

Rumania

German Democratic Republic

USSR

Hungary

Yugos lavia

MISSION

Promote economic and industrial cooperation among the countries with centrally-controlled economies.

ORGANIZATION

CMEA has a Standing Commission on the Use of Atomic Energy for Peaceful Purposes. The Commission holds meetings to review national waste management $R \& D$ programs and defines areas for additional cooperation.

Council for Mutual Economic Assistance

Prospekt Kalinina 56

Moscow

USSR 


\section{EC (EUROPEAN COMMUNITIES)}

MEMBER STATES

\author{
Belgium \\ Denmark \\ France \\ Germany (FRG) \\ Greece
}

Ireland

Italy

Luxembourg

Netherlands

United Kingdom

\section{NUCLEAR MISSIDN}

Promote growth in nuclear industries of member countries, operate joint research center sites, sponsor and fund R\&D, exchange information and establish safety standards.

FUEL CYCLE PRDGRAM ADMINISTRATION

1. Financing--R\&D programs supported by a tax on the member states.

2. Two types of R\&D programs

- Direct action--fully funded by EC, conducted by Euratom Joint Research Centre establishments at Ispra (Italy) and Karlsruhe (FRG)

- Indirect action--partly funded by EC under costsharing contracts, conducted by research centers in the member states.

3. Cooperation with US DOE--waste management cooperative agreement runs from 0ctober 6, 1982 to 0ctober 6, 1987.

\section{ORGANIZATION}

The Commission of the European Communities (CEC) is the executive body for the combined communities (Euratom, Coal and Steel, Common Market).

Commission of the European Communities

200 Rue de la Loi

B-1049 Brussels

Tel: (32) 22351111

Bel gium

Telex: 21877 COMEU B 


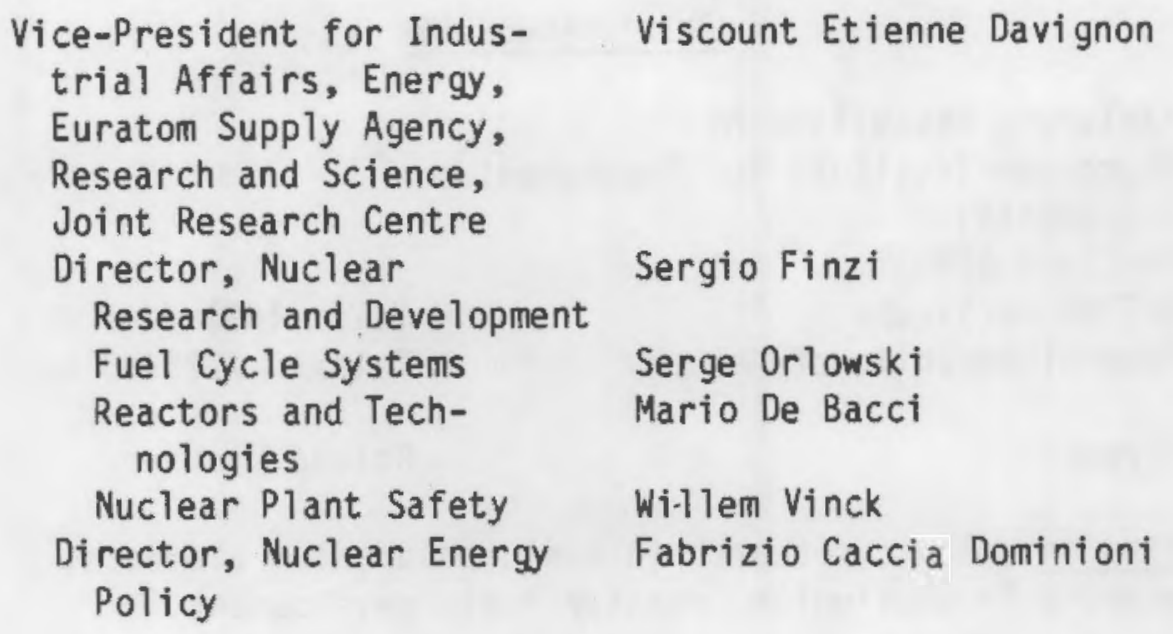

\section{CEC-JRC (JOINT RESEARCH CENTRE)}

Director Genera1, CEC Joint Jean-A1bert Dinkespiler Research Centre (JRC)

\section{JRC: ISPRA}

Ispra Establishment

I-21020 Ispra (Varese) Tel: (39) $332780131 / 780271$ Italy

Telex: $380042 / 38058$ euratom

(Northern Italy; it may be reached by air travel to Milan and ground transport to Ispra, about $50 \mathrm{~km}$.)

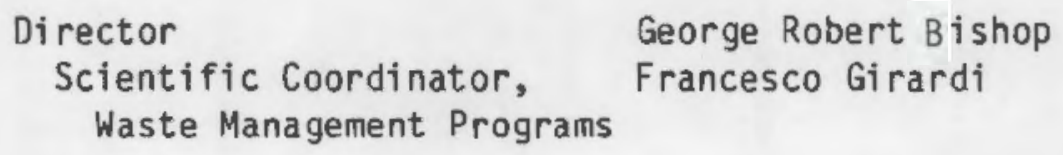

Mission. R\&D in the areas of reactor and nuclear plant safety, treatment and storage of radioactive wastes, standards and reference substances, and training.

Waste Management R\&D. TRU wastes--volume reduction and actinide transmutation; waste disposal--risk analysis, nuclide migration, and waste form properties. 


\section{JRC: KARLSRUHE}

Karlsruhe Establishment

(European Institute for Transuranium

Elements)

Postfach 2266

D-7500 Karlsruhe

Federa1 Republic of Germany

Tel: (49) 7247841

Director

Telex: 7825483 Eu D

Roland Lindner

Mission. Basic research in the transuranium elements, especially plutionium, reactor fuels development.

Fuel Cycle R\&D. Plutonium conversion and plutonium fuels, characterization of waste forms. 


\section{IAEA (INTERNATIONAL ATOMIC ENERGY AGENCY)}

\section{MEMBER STATES}

Over 100 nations (UN members), including USA.

\section{MISSION}

Develop the peaceful use of atomic energy: safeguards, nuclear safety and standards, information exchange, and technical assistance.

\section{Waste Management Activities}

- Development of mutually agreed upon safety standards and criteria for the management and disposal of radioactive waste arising from all stages of the nuclear fuel cycle.

- Promotion of information exchanges in the radioactive waste management area through internationa1 conferences and symposia, technical committees and advisory groups, and specialized training courses and seminars.

- Coordination of research programs in specific technical areas.

- Issuance of publications in the field of waste management, including the annual "Waste Management Research Abstracts."

ORGANIZATION

International Atomic Energy Agency

P.0. Box 100

A-1400 Vienna

Austria

Tel: (43) 22223600

Telex: 112645 
Director General Deputy Director General Director, Division of Nuclear Fuel Cycle Waste Management U.S. National on Waste Management Staff
Hans $B 1 i x$

Boris Semenov

Syed Fareeduddin

J. Hirling

A. Hagan

Int $1-6$ 
ICRP (INTERNATIONAL COMMISSION ON RADIOLOGICAL PROTECTION)

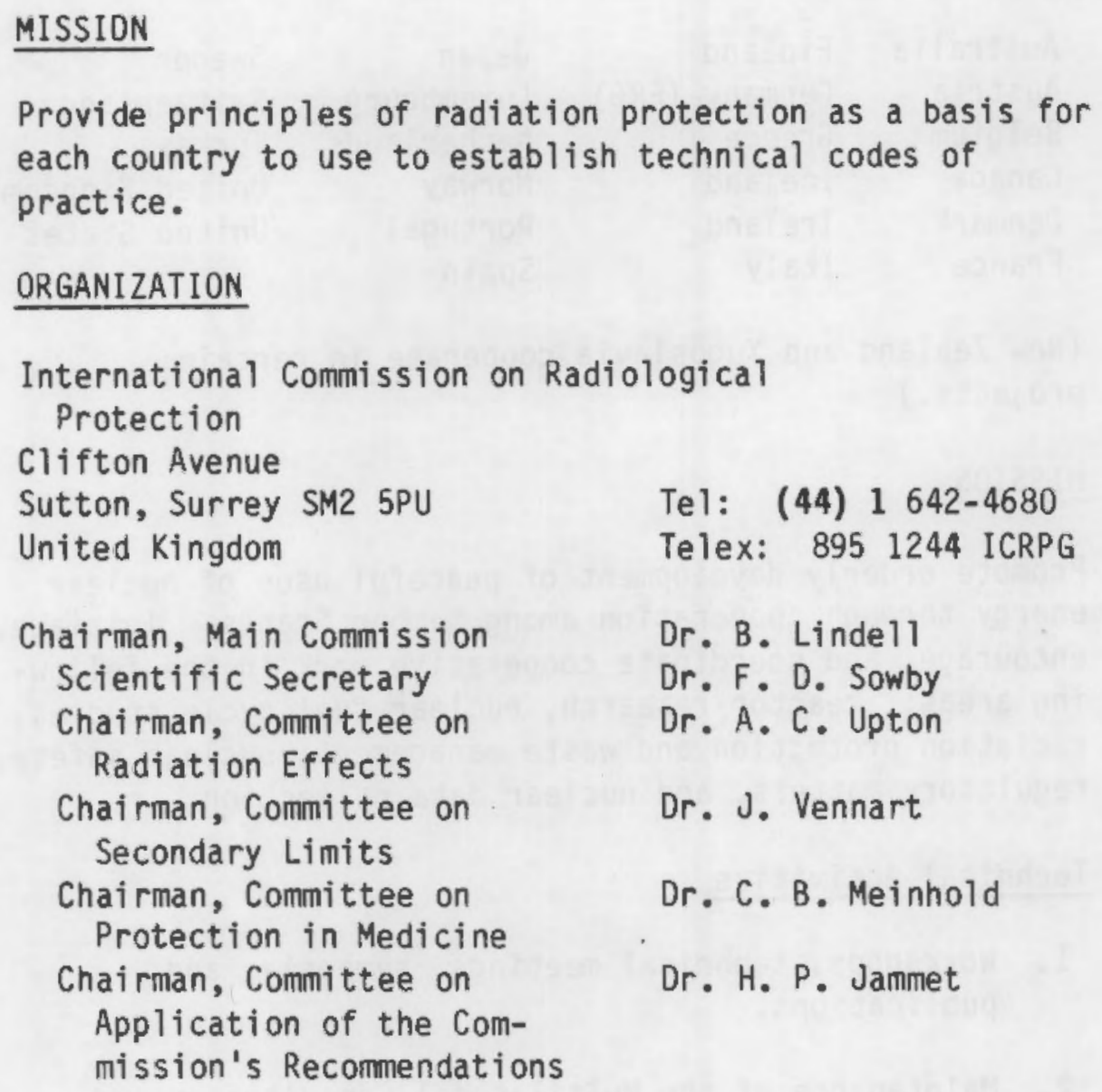


Internationa 1

OECD-NEA (NUCLEAR ENERGY AGENCY)

MEMBER STATES

$\begin{array}{llll}\text { Australia } & \text { Finland } & \text { Japan } & \text { Sweden } \\ \text { Austria } & \text { Germany (FRG) } & \text { Luxembourg } & \text { Switzerland } \\ \text { Belgium } & \text { Greece } & \text { Netherlands } & \text { Turkey } \\ \text { Canada } & \text { Iceland } & \text { Norway } & \text { United Kingdom } \\ \text { Denmark } & \text { Ireland } & \text { Portugal } & \text { United States } \\ \text { France } & \text { Italy } & \text { Spain } & \end{array}$

(New Zealand and Yugoslavia cooperate in certain projects.)

MISSION

Promote orderly development of peaceful uses of nuclear energy through cooperation among Member States. Initiate, encourage, and coordinate cooperative work in the following areas: reactor research, nuclear fuel cycle studies, radiation protection and waste management, nuclear safety, regulatory matters, and nuclear data collection.

Technical Activities

1. Workshops, technical meetings, symposia, and publications.

2. Maintenance of the Multilateral Consultation and Surveillance Mechanism for Sea Disposal, which monitors sea dumping of LLW/ILW in the Northeast Atlantic.

3. Joint projects: International Stripa Mine Project (Sweden); International Sogption Information Retrieval System (ISIRS); Agesta International Decontamination Project (Sweden).

ORGANIZATION

OECD Nuclear Energy Agency

38 Boulevard Suchet

F-75016 Paris

Tel: (33) 15248200

France

Telex: 630668 


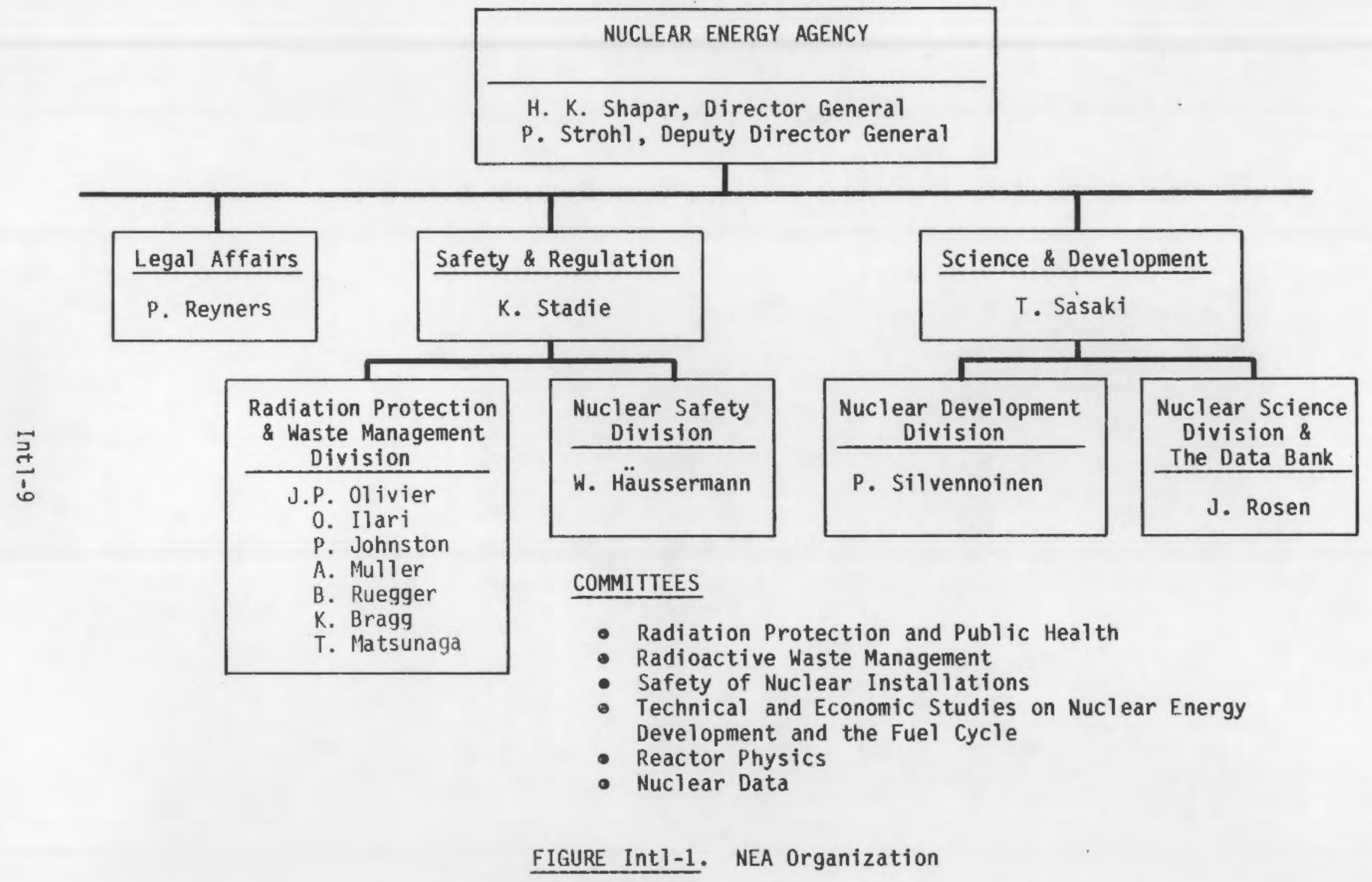





\section{ENERGY SUPPLY AND DEMAND}




\section{ENERGY SUPPLY AND DEMAND}

\section{ENERGY RESERVES}

The world's measured, recoverable, nonrenewable energy reserves (as of 1974) are shown in Figure E-1 and listed by resource in Table E-1. The numbers are relatively old estimates but they do provide an order-of-magnitude picture. (The North American numbers are approximately $1 / 3$ of US-ERDA 1976 estimates for the US alone.)

\section{ENERGY DEMAND}

According to the reference for Figure E-1, in 1974 the world consumed 1 percent of its recoverable, nonrenewable energy resources with the US accounting for one-third of that consumption. In 1978, the world consumed about 250 quads of energy, and the rate of energy use is accelerating, particularly for the developing countries (see Table E-2). The supply form of this 250 quads varied enormous ly from country to

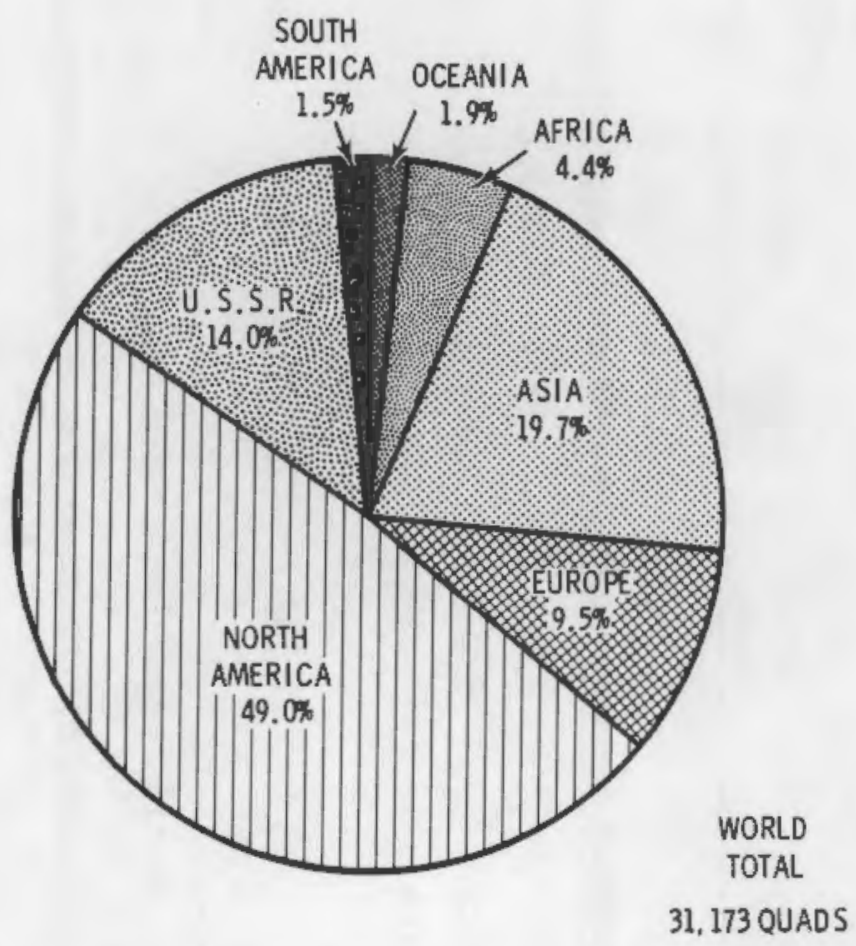

FIGURE E-1. Wor ld Recoverable, Nonrenewable Energy Reserves

Source: Energy Perspectives 2, June 1976, U.S. Department of Interior 
TABLE E-1. Measured World Recoverable Energy Reserves, 1974 (Quads)(a)

\begin{tabular}{|c|c|c|c|c|c|c|}
\hline Area & $\begin{array}{l}\text { Solid } \\
\text { Fuels } \\
\end{array}$ & $\begin{array}{c}\text { Crude } \\
0 \text { il }\end{array}$ & $\begin{array}{c}\text { Natural } \\
\text { Gas } \\
\end{array}$ & $\begin{array}{c}0 i 1 \text { Shale } \\
\text { and } \\
\text { Tar Sands (b) } \\
\end{array}$ & $\begin{array}{c}\text { Uranium } \\
\text { (nonbreeder) }\end{array}(\mathrm{c})$ & Total \\
\hline Africa & 361.7 & 526.6 & 201.7 & 81.4 & 198.1 & $1,359: 5$ \\
\hline Asia (less USSR) & $2,608.7$ & $2,204.4$ & 432.6 & 870.2 & 3.1 & $6,126.0$ \\
\hline Europe (less USSR) & $2,581.5$ & 57.1 & 153.6 & 117.0 & 46.4 & $2,955.6$ \\
\hline North America & $5,070.9$ & 301.0 & 380.6 & $9,111.0$ & 422.7 & $15,286.2$ \\
\hline South America & 49.8 & 311.5 & 60.6 & 23.7 & 11.9 & 457.5 \\
\hline Oceania & 459.8 & 9.4 & 24.9 & 9.2 & 99.1 & 602.4 \\
\hline Total & $11,132.4$ & $3,407.6$ & 1,254 & $10,212.5$ & $>781.3$ & $>26,803.2$ \\
\hline
\end{tabular}

(a) Source: World Energy Conference, Survey of Energy Resources, New York, 1974.

(b) According to the U.S. Department of the Interior, Bureau of Mines, North American tar sands and shale oil reserves may be severely overstated. Development of most of these reserves is not economic at present.

(c) Energy content using breeders 60-100 times as great. Thorium resources neglected. 
TABLE E-2. World Energy Usage (Quads)(a)

\begin{tabular}{|c|c|c|}
\hline & 1980 & 2000 \\
\hline $\begin{array}{l}\text { Industrialized Countries } \\
\text { (including CPEs) }\end{array}$ & 224.8 & $340.4 / 386.1^{(b)}$ \\
\hline Developing Countries & 69.8 & $173.1 / 215.7$ \\
\hline
\end{tabular}

(a) Source: IAEA Book: "Energy, Electricity, and Nuclear Power Estimates Up to 2000," April 1981.

(b) Low/high projections are given.

enormously from country to country. In the major WOCA countries, the supply pattern was as shown in Figure E-2. This figure illustratesthe relatively high dependence on imported oil for Japan, West Germany, and France.

\section{NUCLEAR POWER SUPPLY}

Electricity generation by commercial nuclear power began in the early 1950s in a few highly industrialized countries. From that beginning it has grown to the point where 24 nations (19 WOCA countries) now operate commercial nuclear power plants, and at least a dozen others have active nuclear power programs. Installed electric grid and nuclear power capacities as of the end of 1981 are shown in Table E-3, while Table E-4 provides high/low growth projections into the twenty-first century. Table E-4 utilizes the numbers generated for the INFCE study (1978 estimates) and also lists the nuclear projections obtained in 1980 from NEA Member States in response to a NEA/IAEA questionnaire. The INFCE projections shown in this table are currently too high. For example, the US now estimates a 125 GWe level for the year 2000, as opposed to the 255-395 GWe projection shown in the table.

The relative nuclear power capacities of the countries with major nuclear programs, as of 1979 and as projected to the year 2000, are depicted in Figures E-3 and E-4, respectively. Table E-5 provides low/high estimates of total electrical and nuclear capacities for regions of the world, for 1980 and for the year 2000. 


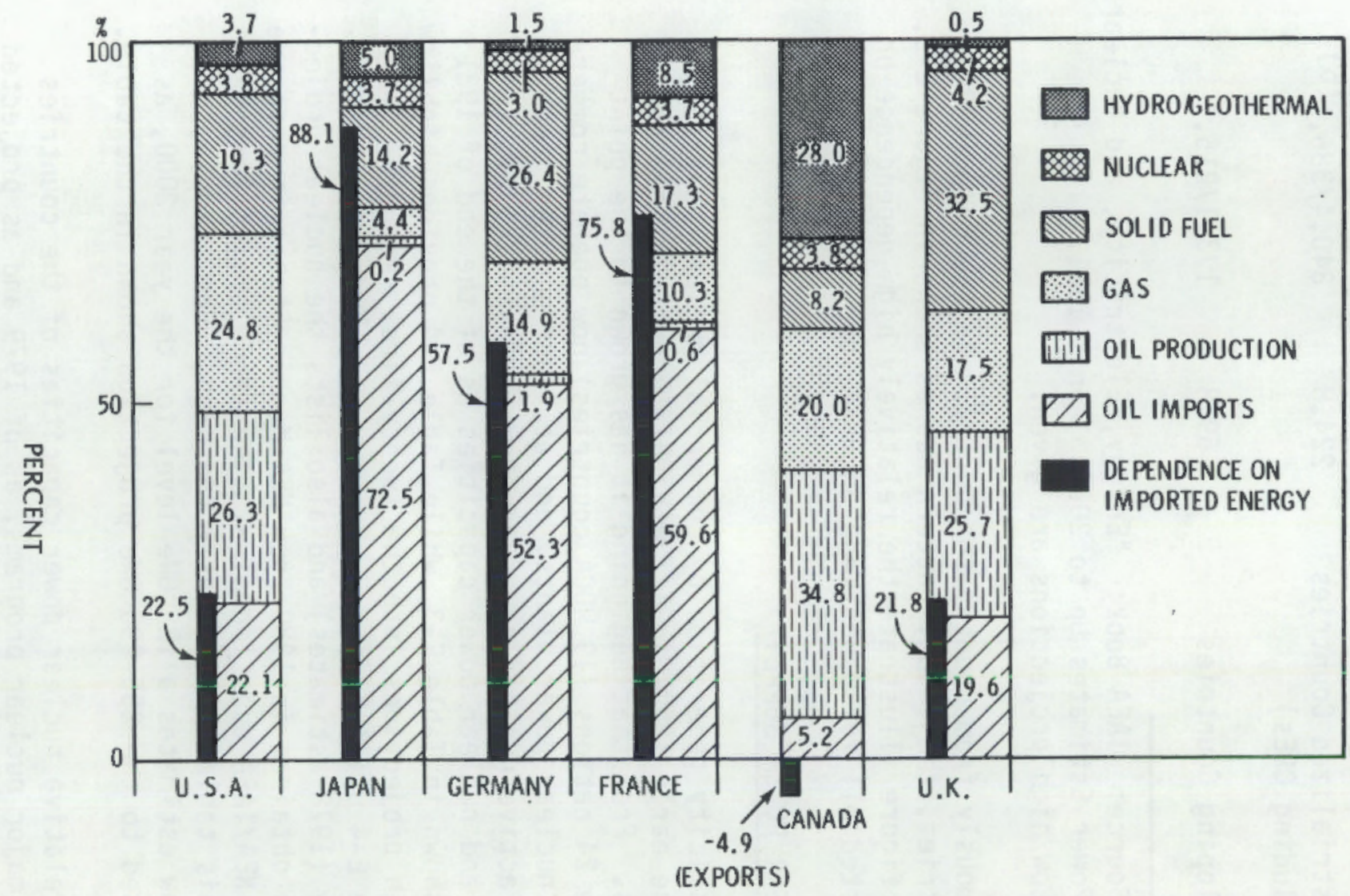

FIGURE E-2. Primary Energy Supply Patterns (1978)

Source: Japan and the 0il Problem, February 1980 
TABLE E-3. Installed Electrical Grid Capacities in Countries with Nuclear Power Programs

\begin{tabular}{|c|c|c|c|c|c|}
\hline \multirow[b]{2}{*}{ Country } & \multirow{2}{*}{$\begin{array}{l}\text { Populations } \\
\text { (millions, } \\
1980)\end{array}$} & \multirow{2}{*}{$\begin{array}{c}\text { Total } \\
\text { Electric } \\
\text { Power Plant } \\
\text { Capacity } \\
\text { (GWe, 1980) } \\
\end{array}$} & \multicolumn{3}{|c|}{$\begin{array}{c}\text { Primary Energy (a) } \\
\text { Demand }\end{array}$} \\
\hline & & & Mtoe & Mtce & Year \\
\hline Argentina & 27 & 13 & 40 & & 1979 \\
\hline Austria & 7.1 & 13 & 27.0 & & 1979 \\
\hline Belgium & 9.8 & 13 & 49.5 & & 1980 \\
\hline Brazil & 119 & 31 & 95 & & 1980 \\
\hline Canada & 23.9 & 79 & 231.3 & & 1980 \\
\hline $\begin{array}{l}\text { China, People's } \\
\text { Republic of }\end{array}$ & 972 & 60 & & 766 & 1978 \\
\hline Egypt & 42 & 4 & & 18.3 & 1978 \\
\hline Finland & 4.8 & 12 & 25.3 & & 1981 \\
\hline France & 53.5 & 73 & 193 & & 1981 \\
\hline $\begin{array}{l}\text { Germany, Federal } \\
\text { Republic of }\end{array}$ & 61.5 & 86 & 257 & & 1982 \\
\hline India & 700 & 29 & 93 & & 1979 \\
\hline Italy & 56.7 & 47 & 142 & & 1980 \\
\hline Japan & 117 & 148 & 386 & & 1980 \\
\hline Korea (South) & 38 & 6 & 41.4 & & 1981 \\
\hline Mexico & $69^{\circ}$ & 17 & 80 & & 1979 \\
\hline Netherlands & 14.1 & 17 & 75.7 & & 1980 \\
\hline Pakistan & 81.5 & 2 & & 13 & 1978 \\
\hline Philippines & 48 & 4.6 & & & \\
\hline South Africa & 28 & 19 & 40.1 & & 1981 \\
\hline Spain & 38.3 & 36 & 73.4 & & 1979 \\
\hline Sweden & 8.4 & 29 & 50.6 & & 1979 \\
\hline Switzerland & 6.3 & 15 & 25.4 & & 1980 \\
\hline Taiwan & 17.7 & 9 & & & \\
\hline United Kingdom & 56 & 82 & 201.5 & & 1980 \\
\hline USA & & 623 & 1853 & & 1980 \\
\hline USSR & 265.5 & 270 & & & \\
\hline
\end{tabular}

(a) 1 Mtoe $=1.6-1.9 \mathrm{Mtce}$ and is equivalent to 3.75 to 4.5 terawatt hrs electric (TWh). 
TABLE E-4. Nuclear Power Growth Projections, GWe (a)

\begin{tabular}{|c|c|c|c|c|c|c|}
\hline \multirow[b]{2}{*}{ Country } & \multicolumn{5}{|c|}{ Nuclear Power Capacity } & \multirow[b]{2}{*}{ Reactor Mix } \\
\hline & 1983 & 1985 & 1990 & 1995 & 2000 & \\
\hline Argentina & 0.3 & 0.9 & 1.6 & 2.3 & 3.0 & PHWR \\
\hline Belgium & 3.5 & 5.4 & 5.4 & 6.7 & 8.0 & PWR \\
\hline Brazil & 0.6 & 0.6 & 1.9 & 3.1 & 4.4 & PWR \\
\hline Bulgaria* & 1.6 & 2.6 & 3.5 & & & PWR \\
\hline Canada & 7.0 & 10.1 & 13.4 & 15.1 & 14.9 & PHWR \\
\hline Cuba* & -- & - & 0.4 & & & PWR \\
\hline Czechos lovakia* & 0.8 & 3.3 & 3.3 & & & PWR \\
\hline Egypt & -- & $\cdots$ & -- & 1.8 & 2.7 & LWR \\
\hline Finland & 2.2 & 2.2 & 2.2 & 3.2 & 3.2 & BWR, PWR \\
\hline France & 24.6 & 35.6 & 51.0 & 57.4 & 61.2 & PWR, GCR, FBR \\
\hline Germany (DR)* & 1.7 & 1.7 & 1.7 & & & PWR \\
\hline Germany (FR) & 9.8 & 16.4 & 22.9 & 25.4 & 28.8 & $\begin{array}{l}\text { BWR, PWR, } \\
\text { HTGR, FBR }\end{array}$ \\
\hline Hong Kong & & & & 1.8 & 1.8 & \\
\hline Hungary* & 0.4 & 1.2 & 1.6 & & & PWR \\
\hline India & 1.0 & 1.2 & 1.9 & 3.0 & 4.4 & BWR, PHWR \\
\hline Italy & 1.3 & 1.3 & 1.3 & 4.8 & 6.7 & BWR, PWR, GCR \\
\hline Japan & 16.6 & 21.2 & 32.0 & 39.6 & 49.8 & $\begin{array}{l}\text { BWR, PWR, } \\
\text { HWR, GCR }\end{array}$ \\
\hline Korea (ROK) & 1.8 & 2.7 & 7.4 & 9.3 & 11.2 & PWR, PHWR \\
\hline Mexico & -- & -- & 1.3 & 1.3 & 1.3 & BWR \\
\hline Netherlands & 0.5 & 0.5 & 0.5 & 0.5 & 0.5 & BWR, PWR \\
\hline Pakistan & 0.1 & 0.1 & 0.1 & 1.1 & 1.1 & PHWR, LWR \\
\hline Philippines & -- & 0.6 & 0.6 & 0.6 & 1.2 & PWR \\
\hline Romania* & & & 1.3 & & & PHWR \\
\hline South Africa & -- & 1.8 & 1.8 & 1.8 & 2.8 & PWR \\
\hline Spain & 2.0 & 5.5 & 7.5 & 8.3 & 10.2 & $\begin{array}{l}\text { BWR, PWR, } \\
\text { GCR }\end{array}$ \\
\hline Sweden & 7.3 & 8.4 & 9.4 & 9.4 & 9.4 & BWR, PWR \\
\hline Switzerland & 1.9 & 2.9 & 2.9 & 3.8 & 3.4 & BWR, PWR \\
\hline Taiwan & 3.1 & 4.0 & 4.9 & 5.8 & 8.7 & BWR, PWR \\
\hline United Kingdom & 6.4 & 10.0 & 10.4 & 10.8 & 13.6 & GCR, PWR, FBR \\
\hline USA & 60.3 & 77.9 & 110.2 & 114.3 & 114.4 & $B W R, P W R, H T R$ \\
\hline USSR* & -- & 25 & $90-100$ & & & PWR, FBR, LGR \\
\hline Yugoslavia & 0.6 & 0.6 & 0.6 & 0.6 & 2.6 & PWR \\
\hline
\end{tabular}

(a) Capacity figures are taken from "NUKEM Market Report on the Nuclear Fuel Cycle," February 1984, unless marked with an asterisk; the latter are taken from Nuclear Engineering International, August 1983, p. 2. 



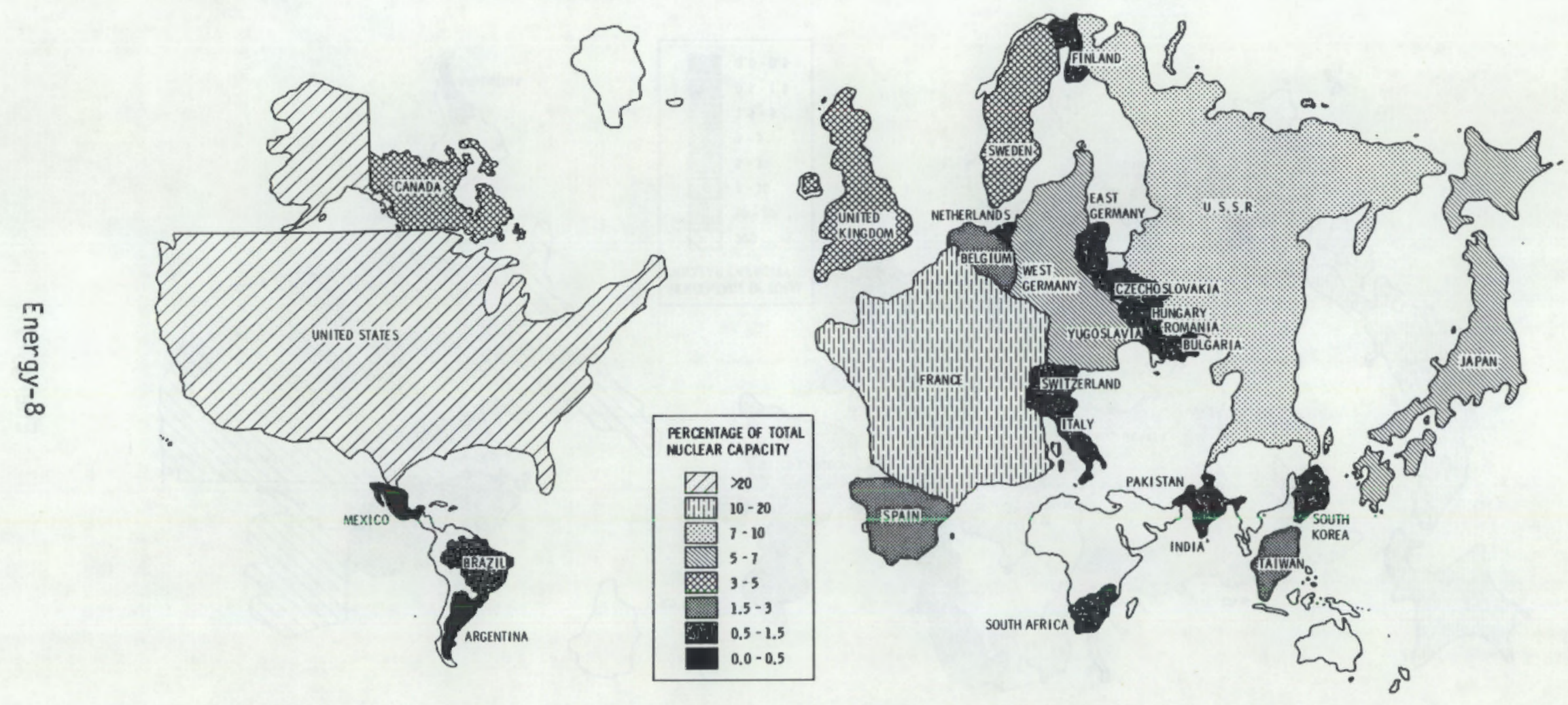

FIGURE E-4. Proportionate World Nuclear Capacity for Named Countries, As of 12/85 Courtesy of HEDL 
TABLE E-5. Estimates of Total and Nuclear Electrical Generating Capacity(a)

\begin{tabular}{|c|c|c|c|c|c|}
\hline & $\cdots$ & 198 & & 2000 & \\
\hline & Country Group & $\begin{array}{l}\text { Total } \\
\text { Electrical } \\
\text { GWe } \\
\end{array}$ & $\begin{array}{c}\text { Nuc lear, } \\
\%\end{array}$ & $\begin{array}{l}\text { Total } \\
\text { Electrical } \\
\text { GWe } \\
\end{array}$ & $\begin{array}{c}\text { Nuc lear, } \\
\%\end{array}$ \\
\hline (1) & OECD North America & 713 & 8.0 & $\begin{array}{l}1213 \\
1600\end{array}$ & $\begin{array}{l}13.9 \\
14.9\end{array}$ \\
\hline (2) & OECD Europe & 441 & 10.1 & $\begin{array}{r}985 \\
1213\end{array}$ & $\begin{array}{l}29.6 \\
29.3\end{array}$ \\
\hline (3) & OECD Pacific & 178 & 8.4 & $\begin{array}{l}437 \\
578\end{array}$ & $\begin{array}{l}22.9 \\
29.4\end{array}$ \\
\hline (4) & $\begin{array}{l}\text { Centrally-Planned } \\
\text { Europe }\end{array}$ & 368 & 4.3 & $\begin{array}{l}1051 \\
1344\end{array}$ & $\begin{array}{r}9.2 \\
15.7\end{array}$ \\
\hline (5) & Asia & 131 & 2.0 & $\begin{array}{r}945 \\
1132\end{array}$ & $\begin{array}{l}4.7 \\
7.1\end{array}$ \\
\hline (6) & Latin America & 101 & 0.3 & $\begin{array}{l}304 \\
386\end{array}$ & $\begin{array}{r}7.6 \\
11.2\end{array}$ \\
\hline (7) & $\begin{array}{l}\text { Africa and } \\
\text { Middle East }\end{array}$ & 63 & & $\begin{array}{l}232 \\
311\end{array}$ & $\begin{array}{l}8.0 \\
8.8\end{array}$ \\
\hline Wor 1 & 1d Total & 1996 & 6.8 & $\begin{array}{l}5167 \\
6554\end{array}$ & $\begin{array}{l}14.4 \\
17.1\end{array}$ \\
\hline $\begin{array}{l}\text { Indu } \\
\text { Cour }\end{array}$ & $\begin{array}{l}\text { ustrialized } \\
\text { ntries }\end{array}$ & 1720 & 7.7 & $\begin{array}{l}3716 \\
4790\end{array}$ & $\begin{array}{l}17.8 \\
20.5\end{array}$ \\
\hline Deve & eloping Countries & 276 & 1.1 & $\begin{array}{l}1451 \\
1774\end{array}$ & $\begin{array}{l}5.7 \\
8.1\end{array}$ \\
\hline
\end{tabular}

(a) Source: IAEA Book "Energy, Electricity and Nuclear Power Estimates for the Period up to 2000," April 1981.

(b) The top and bottom figures are low and high estimates, respectively. 


\section{FAST BREEDER REACTOR STATUS}

The relative status of FBR development worldwide is provided in Figures E-5 and E-6. The US clearly led the world in the initial stages of development. The Experimental Breeder Reactor, EBR-1, provided the first electricity ever generated by nuclear power. The US has maintained its lead in research and development but three countries, France, the Soviet Union and the United Kingdom are now operating large prototype reactors. The US prototype, CRBR, is not expected to be at full power until the end of the decade, at the earliest. A near commercial plant, the 1200 MWe Super Phenix, is scheduled to be connected to the electrical grid in France in 1985.

CONVERSION FACTORS

Tables E-7 and E-8 provide heat content and energy conversion factors. 


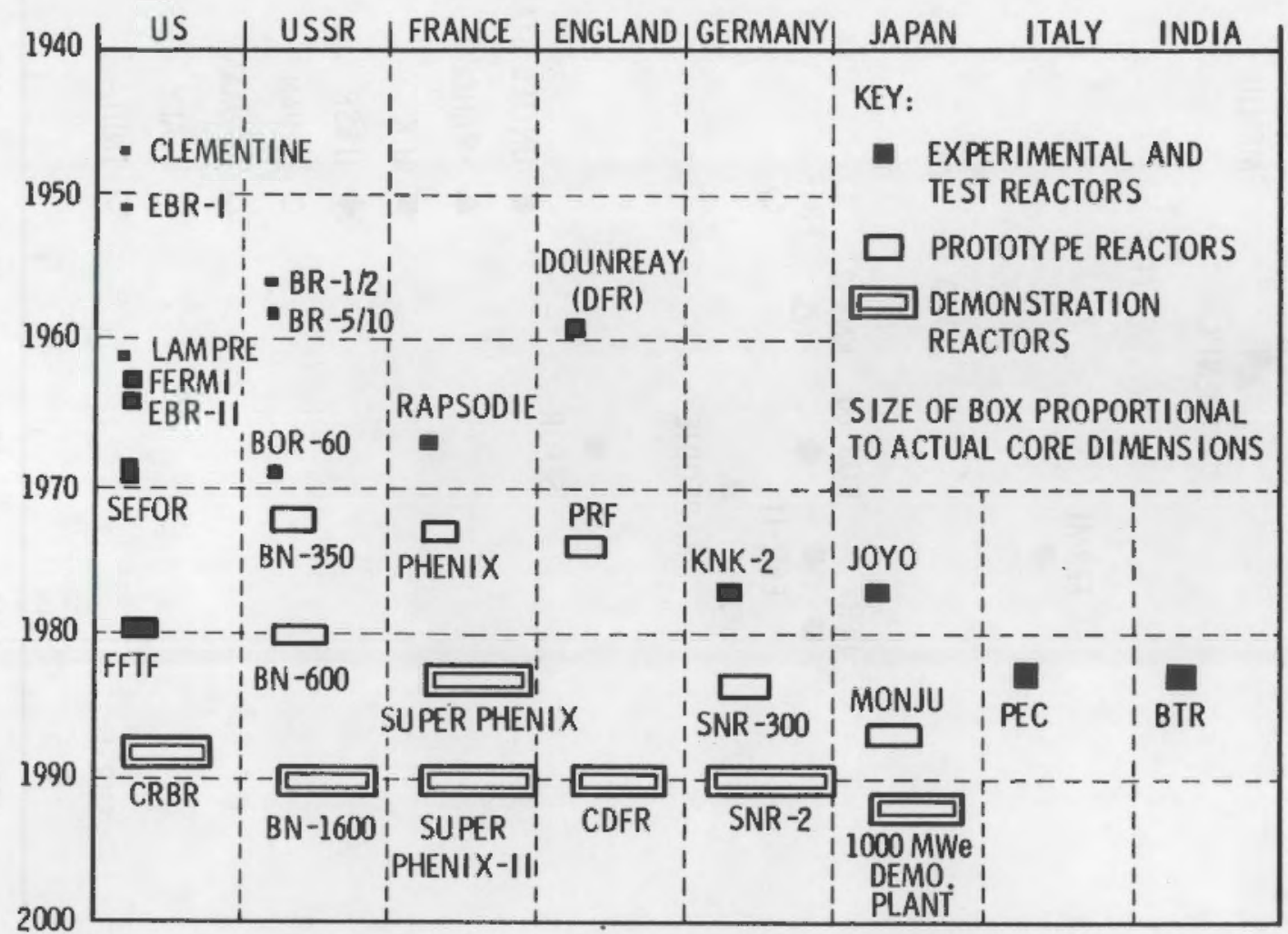

FIGURE E-5. International Fast Reactor Development 


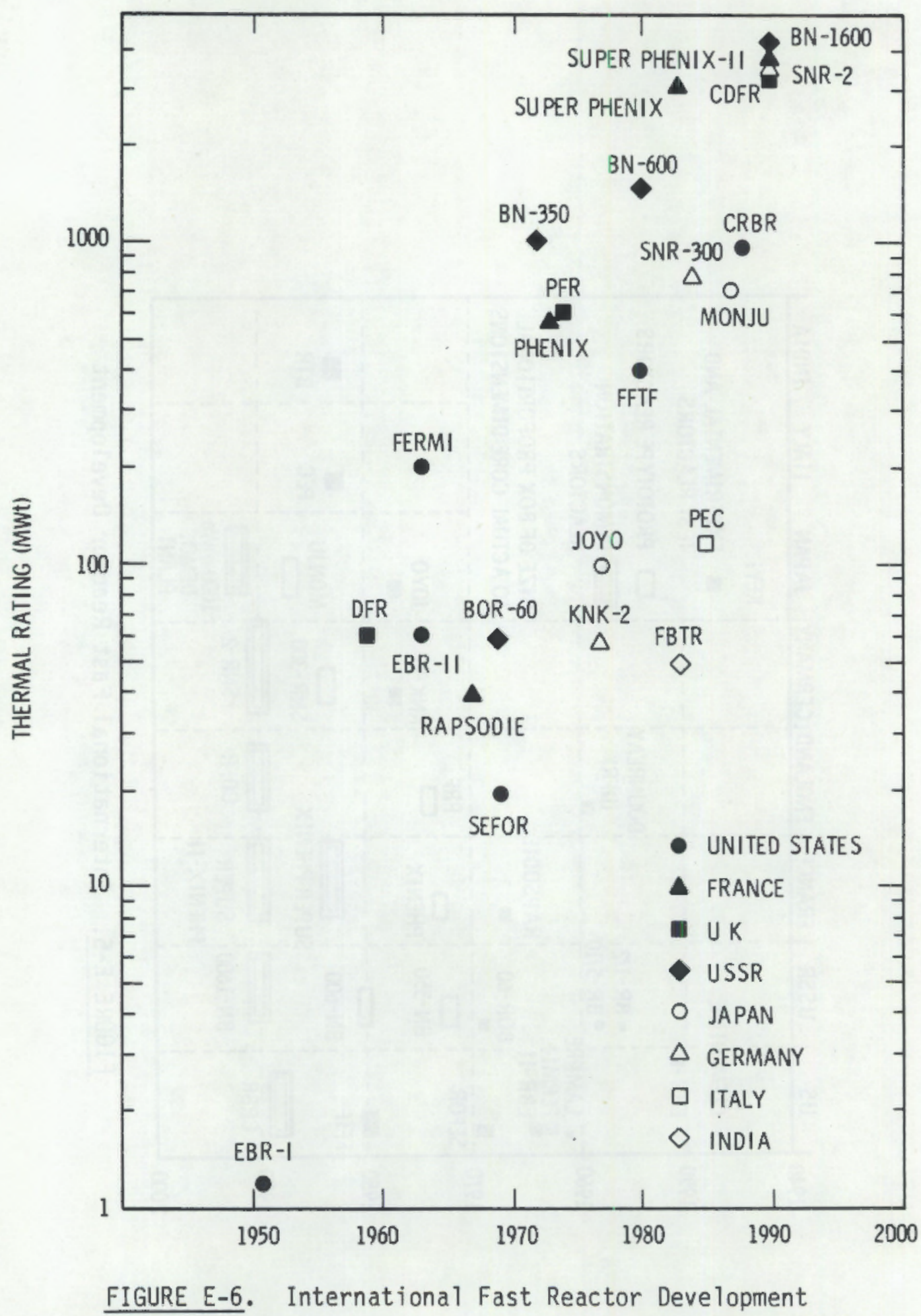

Energy-12 


\section{TABLE E-6. Heat Content Conversion Facțors}

To convert from million tonnes oil equivalent (Mtoe), multiply by the following conversion factors.

Canada U.S. $\underline{\begin{array}{c}\text { OECD } \\ \text { Europe }\end{array}}$

Million tonnes

coal equivalent(a)

Hard Coal

0.69

0.70

0.73

Lignite

0.34

0.20

Terawatt hours (a)

12.5

thermal (TWh[t])

Terawatt hours (a)

electrical (TWh[e])

$\begin{array}{llll}4.22 & 3.86 & 3.9-4.4 & 4.08\end{array}$

Million therms $(b)$

425

Terajoules(b) 44,800

Barrels of oil

1 Barrel = 42 gallons

1 Terawatt hour $=10^{9}$ kilowatt hours

1 Kilowatt hour $=3415 \mathrm{Btu}=8.60 \times 10^{5}$ calories

1 Therm $=10^{5}$ Btu

1 Quad $=10^{15}$ Btu

(a) World Energy Outlook, OECD, 1977.

(b) The World Energy Book, Nichols Publishing Co., 1980. 


\section{TABLE E-7. Energy Conversion}

1 Mile of Supply Train

$\approx 10,000 \mathrm{~T}$ coal

$\approx 50,000$ bbl oil

Quads/Yr (input)

$\approx 1.7$ GWe capacity ( $32 \%$ thermal efficiency $a$

0.63 capacity factor)

1 GWe power plant requires

$\approx 51,000 \mathrm{~T} \mathrm{coal} /$ week (at $32 \%$ thermal efficiency, 100\% capacity)

U.S. coal-fired plants

$\approx 56,900$ miles of supply

Actual coal consumption (1980) train or $569 \times 10^{6} \mathrm{~T}$ coal

U.S. oil-fired plants $\approx 8,420$ miles of supply Actual coal consumption (1980) train or $421 \times 10^{6}$ bbl oil 
FUEL CYCLE 
TABLE F-1. Values of Selected Reactor Parameters(a)

\begin{tabular}{|c|c|c|c|}
\hline Power Station & $\begin{array}{c}\text { Core Power } \\
\text { MWe(net)/MWt }\end{array}$ & $\begin{array}{l}\text { Fuel } \\
\text { Inventory } \\
\text { (Mg HM) } \\
\end{array}$ & $\begin{array}{l}\text { Fuel } \\
\text { Element } \\
\text { Weight } \\
\text { (Mg HM) }\end{array}$ \\
\hline $\begin{array}{l}\text { Tricastin-2 } \\
\text { (PWR-France) }\end{array}$ & $925 / 2785$ & 72.46 & 0.46 \\
\hline $\begin{array}{l}\text { Novo Voronezh } \\
\text { (PWR-USSR) }\end{array}$ & $420 / 1375$ & 42 & 0.12 \\
\hline $\begin{array}{l}\text { Krümmel KKK } \\
\text { (BWR-FRG) }\end{array}$ & $1260 / 3690$ & 155.8 & 0.185 \\
\hline $\begin{array}{l}\text { Bruce } 4 \\
\text { (PHWR-C anada) }\end{array}$ & $740 / 2515$ & 115 & 0.018 \\
\hline $\begin{array}{l}\text { Wylfa } 1 \\
\text { (GCR-UK) }\end{array}$ & $420 / 1650$ & 595 & 0.012 \\
\hline $\begin{array}{l}\text { Hinck ley Point Bl } \\
\text { (AGR-UK) }\end{array}$ & $621 / 1494$ & 113.7 & 0.046 \\
\hline $\begin{array}{l}\text { Super Phenix } \\
\text { (LMFBR-France) }\end{array}$ & $1200 / 3000$ & 32 & 0.089 \\
\hline
\end{tabular}

(a) Information gathered from Nuclear Engineering International, July/August 1980 supplement; "World List of Nuclear Power Plants, Nuclear News, June 30 , 1980; and Power Reactors in Member States, International Atomic Energy Agency, 1978. 
TABLE F-2. Values of Selected Spent Fuel Parameters(a)

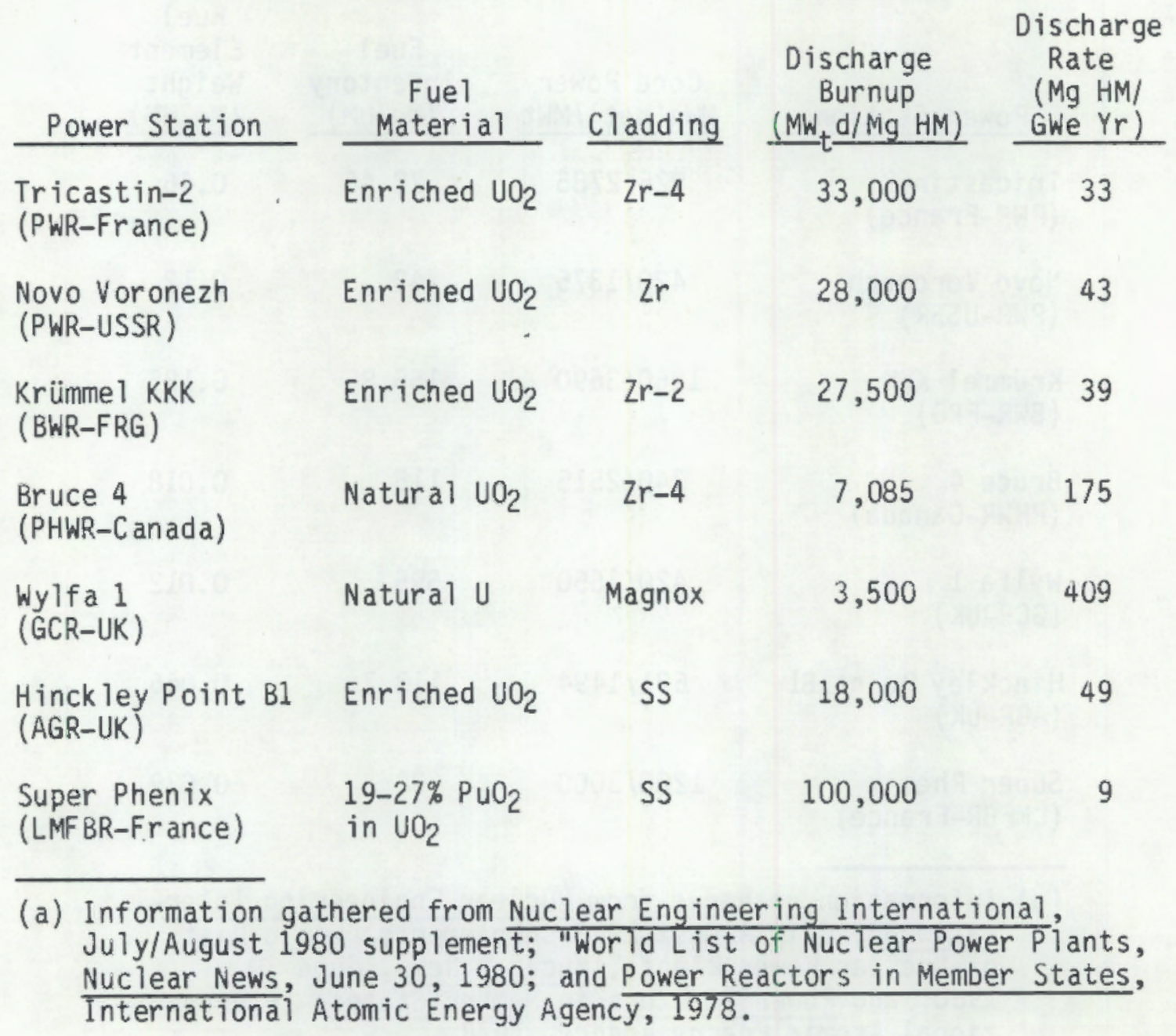

Fuel-2 
TABLE F-3. Forecast of Spent Fuel Arisings(a)

\begin{tabular}{|c|c|c|c|c|}
\hline \multirow[b]{2}{*}{ Country } & \multicolumn{4}{|c|}{ Fuel Discharged (cumulative $t U$ ) } \\
\hline & 1980 & 1985 & 1990 & 2000 \\
\hline Argentina & 416 & 940 & 1,900 & 5,800 \\
\hline Belgium & 196 & 560 & 1,290 & 3,000 \\
\hline Brazil & -- & 32 & 180 & 1,000 \\
\hline Canada & 3,650 & 8,800 & 17,700 & 38,000 \\
\hline Egypt & -- & -- & -- & 360 \\
\hline Finland & 48 & 350 & 650 & 1,400 \\
\hline France & 248 & 2,700 & 7,700 & 22,000 \\
\hline Germany (FR) & 960 & 2,250 & 4,550 & 11,000 \\
\hline Hong Kong & & & & 250 \\
\hline India & 370 & 780 & 1,580 & 5,000 \\
\hline Italy & 160 & 330 & 520 & 2,000 \\
\hline Japan & 1,450 & 4,100 & 8,200 & 20,000 \\
\hline Korea (ROK) & 17 & 360 & 1,450 & 4,400 \\
\hline Mexico & -- & -- & 100 & 500 \\
\hline Netherlands & 103 & 190 & 270 & 420 \\
\hline Pakistan & 49 & 110 & 170 & 440 \\
\hline Philippines & -- & -- & 80 & 270 \\
\hline South Africa & -- & 40 & 310 & 1,200 \\
\hline Spain & 176 & 490 & 1,300 & 3,400 \\
\hline Sweden & 465 & 1,330 & 2,360 & 5,000 \\
\hline Switzerland & 380 & 650 & 1,090 & 2,000 \\
\hline Taiwan & 70 & 430 & 1,140 & 2,600 \\
\hline United Kingdom & 250 & 830 & 2,000 & 5,300 \\
\hline USA & 8,989 & 13,811 & 27,073 & 57,886 \\
\hline Yugoslavia & -- & 50 & 130 & 420 \\
\hline
\end{tabular}

(a) Only $\mathrm{UO}_{2}$ fuels from LWRs, British AGRs, HWRs and Russian oxide-fueled graphite-moderated reactors are included.

For all countries except the US, the spent fuel forecasts are estimated from data in NAC Reports C-8023, "Worldwide Spent Fuel Disposition Analysis," September 1980; and T-8105, "Light-Water Reactor Spent Fuel Storage and Reprocessing in Europe in the 1.980s, "December 1981; corrected to reflect current projections of nuclear power plant capacity.

US data is from "Spent Fuel and Radioactive Waste Inventories, Projections and Characteristics," DOE/NE-0017-2, May 1983. 
TABLE F-4. Spent Fuel Reprocessing Activities and HLW Inventories

\begin{tabular}{|c|c|c|c|}
\hline Country (Site) & $\begin{array}{c}\text { Operation } \\
\text { Dates }\end{array}$ & $\begin{array}{c}\text { Fuel } \\
\text { Reprocessed } \\
\end{array}$ & $\begin{array}{c}\text { HLW } \\
\text { Inventory }\end{array}$ \\
\hline $\begin{array}{l}\text { Eurochemic } \\
\qquad \text { (Mol, Belgium) }\end{array}$ & $1966-1974$ & $\begin{array}{c}1,360 \mathrm{~kg} \mathrm{HEU} \\
181 \mathrm{t} \text { LEU }\end{array}$ & $\begin{array}{r}800 \mathrm{~m}^{3} \text { HEWC } \\
67 \mathrm{~m}^{3} \text { LEWC }\end{array}$ \\
\hline Canada & -1979 & Lab studies & $16 \mathrm{~m}^{3}$ \\
\hline FRG (WAK) & $1971-1980$ & $\begin{array}{l}100 t \\
(43 t \text { LWR })\end{array}$ & $65 \mathrm{~m}^{3}$ \\
\hline $\begin{aligned} \text { France } & (\text { UP }-1) \\
& \text { (AVM) }\end{aligned}$ & $\begin{array}{l}1958-1983 \\
6 / 78-3 / 83\end{array}$ & $12,000 \mathrm{t}(\mathrm{GCR})$ & $\begin{array}{l}286 \text { t HLW } \\
\text { glass ( } 836 \\
\text { canisters) }\end{array}$ \\
\hline $\begin{array}{c}\text { France (UP-2, } \\
\text { La Hague) }\end{array}$ & $\begin{array}{l}1966-1977 \\
1976-1983\end{array}$ & 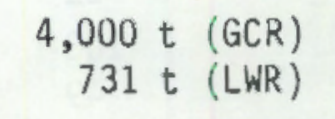 & \\
\hline $\begin{array}{r}\text { India (Trombay) } \\
\text { (Tarapur) }\end{array}$ & $\begin{array}{l}1965-1974 \\
1982-\end{array}$ & & \\
\hline $\begin{array}{l}\text { Italy } \\
\text { (Saluggia) } \\
\text { (Trisaia) }\end{array}$ & & & $\begin{array}{r}100 \mathrm{~m}^{3} \\
3 \mathrm{~m}^{3}\end{array}$ \\
\hline Japan (Tokai) & $1977-1983$ & $170 t$ & \\
\hline United Kingdom & $\begin{array}{l}1979 \\
1952-1980 \\
1969-1973\end{array}$ & 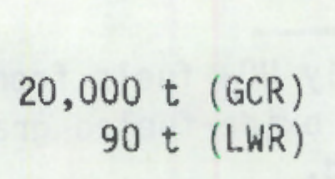 & $1000 \mathrm{~m}^{3}$ \\
\hline $\begin{array}{l}\text { United States } \\
\text { (Nuclear Fuel } \\
\text { Services) }\end{array}$ & $1966-1971$ & $\begin{array}{l}244 \text { t oxides } \\
375 \text { t metal }\end{array}$ & $2100 m^{3}$ \\
\hline
\end{tabular}


TABLE F-5. Foreign Fuel Reprocessing Contracts (tHM)

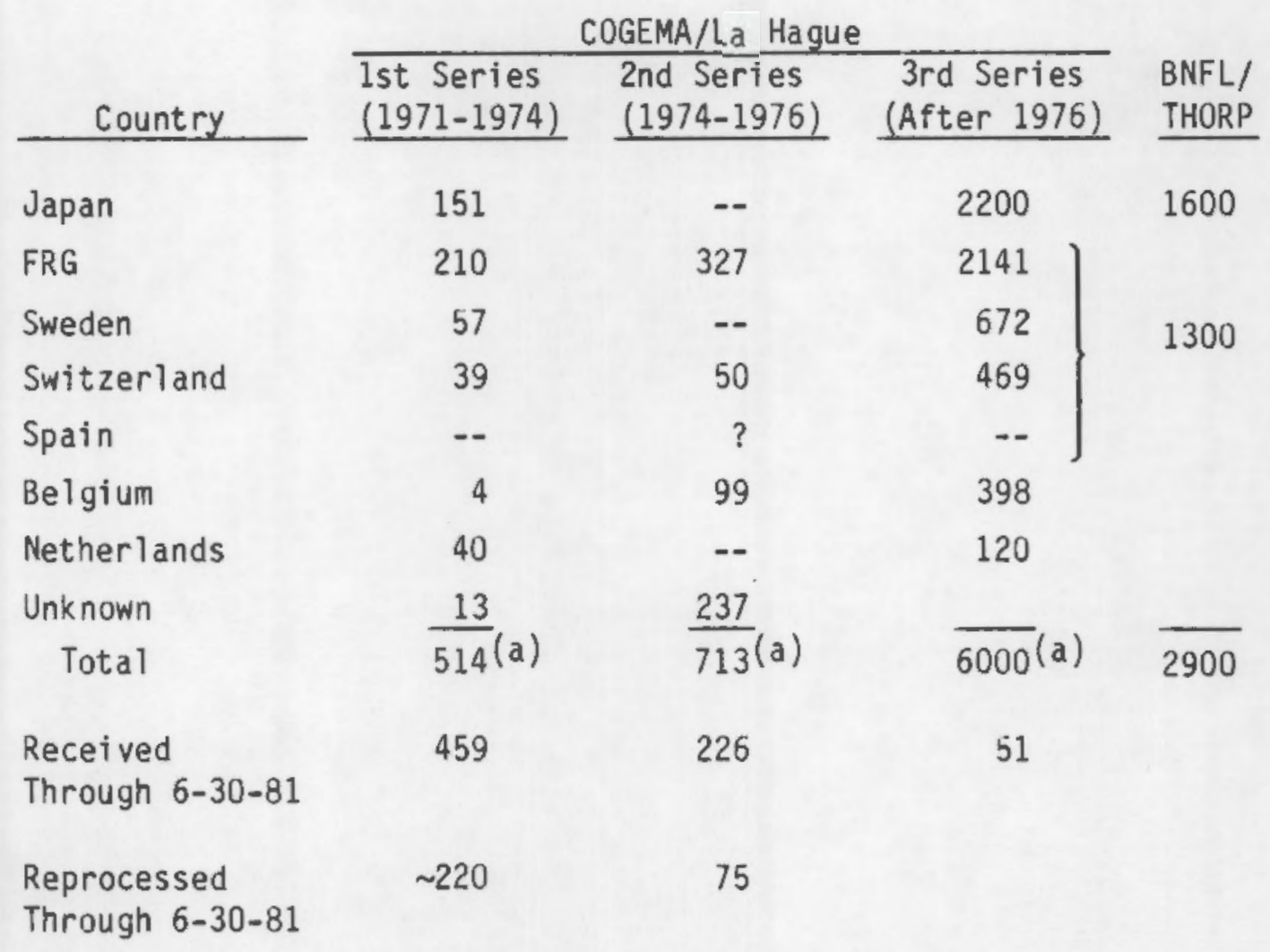

(a) Total contracted by COGEMA--each series under different terms. Reference: The Energy Daily, September 10, 1981. 

TRAVEL AIDS 


\section{INTERNATIONAL DIALING INSTRUCTIONS}

Telephone numbers in the International Nuclear Fuel Cycle Fact Book show country code, city code and local number, however, direct dial is not available in all areas and operator assistance may be necessary.

\begin{tabular}{|l|} 
TO DIAL \\
OIRECT
\end{tabular} $011+\begin{gathered}\text { COUNTRY } \\
\text { CODE } \\
2-3 \text { Digits }\end{gathered}+$\begin{tabular}{|c|} 
CITY \\
COOE \\
$1-5$ Digits
\end{tabular}$+$\begin{tabular}{|l} 
LOCAL \\
NUMBER \\
$2-9$ Digits
\end{tabular}

\begin{tabular}{|c|c|c|}
\hline TO DIAL WITH \\
OPERATOR \\
ASSISTANCE
\end{tabular}$\quad 0+\quad \begin{gathered}\text { REQUEST THE OVERSEAS } \\
\text { OPERATOR FOR THE COUNTRY } \\
\text { YOU ARE CALLING }\end{gathered}$

Dial "0" and ask for an overseas operator to obtain:

- numbers

- country and city codes

- rates

- other assistance.

Points to remember:

- It may take up to 45 seconds for the overseas phone to ring

- Overseas ringing and busy signals may vary

- There are time differences

- Rate discounts may apply. Call the overseas operator for information. 


\section{INTERNATIONAL STANDARD TIME CHART}

\begin{tabular}{|c|c|c|}
\hline & $\begin{array}{l}\text { Hours From } \\
\text { Wash., DC } \\
\end{array}$ & $\begin{array}{c}\text { Daylight Saving Time } \\
\text { Effective Period } \\
\text { (first and last day) }\end{array}$ \\
\hline Argentina & +2 & -- \\
\hline \multicolumn{3}{|l|}{ Australia } \\
\hline (New South Wales) & $+151 / 2$ & $10 / 30 / 83-03 / 03 / 84$ \\
\hline Austria & +6 & $03 / 25-09 / 29 / 84$ \\
\hline Belgium & +6 & $03 / 25-09 / 29 / 84$ \\
\hline $\begin{array}{l}\text { Brazil } \\
\quad \text { East-including }\end{array}$ & & \\
\hline all coast) & +2 & - \\
\hline \multicolumn{3}{|l|}{ Canada } \\
\hline (At lantic) & +1 & \\
\hline (Eastern) & 0 & $04 / 29-10 / 07 / 84$ \\
\hline (Pacific) & -3 & \\
\hline China (People's Republic) & +13 & $10 / 03 / 83-03 / 10 / 84$ \\
\hline Denmark & +6 & $03 / 25-09 / 29 / 84$ \\
\hline Finland & +7 & $03 / 25-09 / 29 / 84$ \\
\hline France & +6 & $03 / 25-09 / 29 / 84$ \\
\hline Germany (FRG) & +6 & $03 / 25-09 / 29 / 84$ \\
\hline India & $+10 \frac{1}{2}$ & -- \\
\hline Italy & +6 & $03 / 25-09 / 29 / 84$ \\
\hline Japan & +14 & -- \\
\hline Korea (Republic of) & +14 & -- \\
\hline \multicolumn{3}{|l|}{ Mexico } \\
\hline (Baja Calif. Norte) & -3 & $04 / 29-10 / 27 / 84$ \\
\hline Netherlands & +6 & $03 / 25-09 / 29 / 84$ \\
\hline Pakistan & +10 & -- \\
\hline South Africa & +7 & -- \\
\hline Spain & +6 & $03 / 25-09 / 29 / 84$ \\
\hline Sweden & +6 & $03 / 25-09 / 29 / 84$ \\
\hline Switzerland & +6 & $03 / 25-09 / 29 / 84$ \\
\hline Taiwan (Republic of China) & +13 & -- \\
\hline \multicolumn{3}{|l|}{ USA } \\
\hline (Central) & -1 & \\
\hline (Mountain) & -2 & \\
\hline (Pacific) & -3 & $04 / 29-10 / 27 / 84$ \\
\hline (Alaska) & -4 & \\
\hline (Hawaii) & -5 & \\
\hline USSR (Moscow time) & +8 & $04 / 01-09 / 30 / 84$ \\
\hline United Kingdom & +5 & $03 / 25-10 / 27 / 84$ \\
\hline
\end{tabular}




\section{PASSPORTS}

Passports are needed to depart and re-enter the United States. To obtain current information and passport application/renewal forms contact your county courthouse. Most travel agencies also provide current information and necessary forms.

$\underline{\text { VISAS }}$

While many foreign countries no longer have visa requirements for U.S. citizens, some still do. Most travel agencies can provide up-to-date information on visa requirements as well as the necessary forms. It is necessary to obtain most visas prior to departure, and they should be obtained well in advance from the nearest consulate of the country to be visited.

Visas are currently required for visits to the following countries:

- Argentina

- Australia

- Brazil

- China (People's Republic)

- India

- Japan

- Korea (Republic of)

- Mexico

- Pakistan

- South Africa

- Taiwan (Republic of China)

- USSR 


\section{CURRENCY EXCHANGE RATES}

The following table illustrates the US dollar equivalent on 3-7-84. (a) As rates fluctuate daily, it is recommended that current rates be obtained from local banks or newspapers prior to departure.

US \$

\begin{tabular}{|c|c|c|}
\hline Country & Currency & Equivalent \\
\hline Argentina & Peso & 0.034 \\
\hline Australia & Dollar & 0.95 \\
\hline Austria & Shilling & 0.055 \\
\hline Belgium & Franc & 0.019 \\
\hline Brazil & Cruzeiro & 0.008 \\
\hline Canada & Dollar & 0.79 \\
\hline China (People's Republic) & Yuan & 0.49 \\
\hline Denmark & Krone & 0.10 \\
\hline Finland & Markka & 0.18 \\
\hline France & Franc & 0.12 \\
\hline Germany (FRG) & Mark & 0.39 \\
\hline India & Rupee & 0.09 \\
\hline Italy & Lira & 0.0006 \\
\hline Japan & Yen & 0.004 \\
\hline Korea (South) & Won & 0.0012 \\
\hline Netherlands & Guilder & 0.34 \\
\hline South Africa & Rand & 0.84 \\
\hline Spain & Peseta & 0.0067 \\
\hline Sweden & Krona & 0.13 \\
\hline Switzerland & Franc & 0.47 \\
\hline Taiwan (Republic of China) & Dollar & 0.024 \\
\hline United Kingdom & Pound & 1.47 \\
\hline
\end{tabular}

(a) Wall Street Journal, 3/8/84. 


\section{DISTRIBUTION}




\section{DISTRIBUTION}

No. of

Copies

OFFSITE

DOE HEADQUARTERS

X. D. Izel1, GC-42

Office of the General Counsel

U.S. Department of Energy

Forrestal

Washington, DC 20585

S. K. Kuznick, GC-32

office of the General Counsel

U.S. Department of Energy Forrestal

Washington, OC 20585

S. N. Ceja, IA-21

U.S. Department of Energy Forrestal

Washington, DC 20585

J. A. Dugger, IA-22

U.S. Department of Energy

Forrestal

Washington, DC 20585

S. Griffith, IA-22

U.S. Department of Energy

Forrestal

Washington, DC 20585

H. Jaffe, IA-20

U.S. Department of Energy Forrestal

Washington, DC 20585

M. D. MCDonough, IA-21

U.S. Department of Energy Forrestal

Washington, DC 20585

T. K. Lau, IA-22

U.S. Department of Energy

Forrestal

Washington, DC 20585

W. Porter, $[A-2]$

U.S. Department of Energy

Forrestal

Washington, DC 20585

2 R. E. Robertson, $I A-21$

U.S. Department of Energy Forrestal

Washington, DC 20585
No. of

Copies

K. W. Hentzel, $I A-20$

U.S. Department of Energy

Forrestal

Washington, DC 20585

A. K. Flieger, $\mathrm{DP}-333$

U.S. Department of Energy

Forrestal

Washington, DC 20585

R. F. Kott, $D P-333$

U.S. Department of Energy

Forrestal

Washington, DC 20585

R. L. Morgan, $D P-1$

U.S. Department of Energy Forrestal

Washington, DC 20585

R. D. Nelson, DP-331

U.S. Department of Energy

Forrestal

Washington, DC 20585

G. H. Daly, DP-10

U.S. Department of Energy

GTN

Washington, DC 20545

J. E. Dieckhoner, DP-122

U.S. Department of Energy GTM

Washington, DC 20545

R. K. Heusser, DP- 132

U.S. Department of Energy GTN

Washington, DC 20545

J. J. Jicha, DP-123

U.S. Department of Energy GTN

Washington, DC 20545

D. B. LeC1aire, DP-12

U.S. Department of Energy GTN

Washington, DC 20545

R. S. Scott, $D P-121$

U.S. Department of Energy GTN

Washington, DC 20545
No. of

Copies

A. L. Taboas, OP-123

U.S. Department of Energy GTN

Washington, DC 20545

R. D. Walton, Jr, $D P-123$

U.S. Department of Energy GTN

Washington, DC 20545

L. R. Willett, OP-132

U.S. Department of Energy GTN

Washington, DC 20545

S. T. Brewer, NE-1

U.S. Department of Energy Forrestal

Washington, DC 20585

T. A. Dillon, NE-2

U.S. Department of Energy Forrestal

Hashington, DC 20585

P. A. Garon, NE-72

U.S. Department of Energy Forrestal

Washington, DC 20585

2 S. Rosen, $N E-75$

U.S. Department of Energy Forrestal

Hashington, DC 20585

D. E. Bailey, NE-42

U.S. Department of Energy GTN

Washington, DC 20545

W. W. Ballard, Jr, NE-22 U.S. Department of Energy GTN

Washington, DC 20545

C. B. Bastin, Jr, NE-42

U.S. Department of Energy GTN

Washington, DC 20545

J. E. Baublitz, NE-24

U.S. Department of Energy GTN

Washington, DC 20545 
No. of

Copies

R. B. Chitwood, NE-43

U.S. Department of Energy GTN

Washington, DC 20545

2 F. E. Coffman, $N E-20$

U.S. Department of Energy GTN

Washington, DC 20545

2 J. A. Coleman, NE-25

U.S. Department of Energy GTN

Washington, DC 20545.

R. Coleman, NE-22

U.S. Departinent of Energy GTN

Washington, DC 20545

E. G. Del aney, NE-24

U.S. Department of Energy GTN

Washington, DC 20545

N. Goldenberg, NE-74

U.S. Department of Energy GTN

Washington, OC 20545

F. Goldner, NE-75

U.S. Department of Energy GTN

Washington, DC 20545

24 K. E. Horton, NE-75

U.S. Department of Energy GTN

Washington, DC 20545

E. A. Jordan, NE-25

U.S. Department of Energy GTN

Washington, DC 20545

K. 0. Laughon, NE-40

U.S. Department of Energy GTN

Washington, DC 20545

D. J. McGoff, NE-23

U.S. Department of Energy GTN

Washington, DC 20545

W. H. McVey, NE-42

U.S. Department of Energy GTN

Washington, OC 20545
No. of

Copies

H. H. Rohm, NE-50

U.S. Department of Energy GTN

Washington, DC 20545

R. H. Bauer, RW-30

U.S. Department of Energy Forrestal

Washington, DC 20585

K. A. Klein, RW-31

U.S. Department of Energy Forrestal

Washington, DC 20585

M. J. Lawrence, RW-2

U.S. Department of Energy Forrestal

Washington, DC 20585

11 A. F. Perge, RW-3

U.S. Department of Energy

Forrestal

Washington, DC 20585

R. Stein, RW-21

U.S. Department of Energy Forrestal

Washington, OC 20585

J. W. Bennett, RW-20

U.S. Department of Energy GTN

Washington, OC 20545

C. R. Cooley, RW-24

U.S. Departinent of Energy GTN

Washington, DC 20545

J. J. Fiore, RH-22

U.S. Department of Energy GTN

Washington, DC 20545

B. Hill

Tokyo Office, American Embassy

U.S. Department of Energy

c/o U.S. Department of State

Washington, DC 20520

DOE OPERATIONS

D. G. Boyer

Albuquerque Operations office

U.S. Department of Energy

P.0. Box 5400

Albuquerque, NM 87115
No. of

Copies

D. Campbell

Albuquerque Operations Office

U.S. Department of Energy

P.0. Box 5400

Albuquerque, NM 87115

W. W. Cooper (WIPP

Project office)

Albuquerque Operations office

U.S. Department of Energy

P.0. Box 5400

Albuquerque, NM 87115

5 J. McGough

Albuquerque Operations office

U.S. Department of Energy

P.0. Box 5400

Albuquerque, NM 87115

J. Morley

Albuquerque Operations 0 ffice

U.S. Department of Energy P.0. Box 5400

Albuquerque, NM 87115

D. T. Schueler

Albuquerque Operations office

U.S. Department of Energy P.0. Box 5400

Albuquerque, NM 87115

H. J. Rauch (Manager)

Chicago Operations Office

U.S. Department of Energy

9800 South Cass Avenue

Argonne, IL 60439

S. A. Mann

Chicago Operations Office U.S. Department of Energy 9800 South Cass Avenue Argonne, IL 60439

M. Bl anchard

National Waste Program office

U.S. Department of Energy

$505 \mathrm{King}$ Avenue

Columbus, $\mathrm{OH} 43201$

L. Casey

National Waste Proyram office

U.S. Department of Energy

$505 \mathrm{King}$ Avenue

Columbus, $O H 43201$ 
No. of

Copies

D. E. Cattran

National Waste Program office

U.S. Department of Energy

$505 \mathrm{King}$ Avenue

Columbus, $\mathrm{OH} 43201$

J. Engl and

National Waste Program office

U.S. Department of Energy

$505 \mathrm{King}$ Avenue

Columbus, OH 43201

I. Lahoti

National Waste Program office

U.S. Department of Energy

$505 \mathrm{King}$ Avenue

Columbus, $\mathrm{OH} 43201$

J. 0. Neff

National Waste Program office

U.S. Department of Energy

$505 \mathrm{King}$ Avenue

Columbus, OH 43201

P. Vanloan

National Waste Program office

U.S. Department of Energy

$505 \mathrm{King}$ Avenue

Columbus, OH $\mathbf{4 3 2 0 1}$

R. Wunderlich

National Waste Program Office

U.S. Department of Energy

$505 \mathrm{King}$ Avenue

Columbus, $\mathrm{OH} \quad 43201$

H. J. Argyle

Idaho Operations office

U.S. Department of Energy

550 Second Street

Idaho Falls, I0 83401

M. J. Barainca

Idaho Operations office

U.S. Department of Energy

550 Second Street

Idaho Falls, ID 83401

E. Maestas

Idaho Operations Office

U.S. Department of Energy

550 Second Street

Idano Falls, 1083401
No. of

Copies

R. Nelson

Idaho Operations office

U.S. Department of Energy

550 second Street

Idaho Falls, ID 83401

T. E. Wade, II (Manager)

Idaho Operations office

U.S. Department of Energy

550 second Street.

Idaho Falls, I0 83401

C. P. Gertz

Idaho Operations office

U.S. Department of Energy

550 Second Street

Idaho Falls, ID 83401

R. D. Duncan

Nevada Operations Office

U.S. Department of Energy

Las Vegas, NV 89114-4100

A. R. Hakl

Nevada Operations Office

U.S. Department of Energy

Las Vegas, NV 89114-4100

D. L. Vieth

Nevada Operations Office

U.S. Department of Energy

Las Vegas, NV 89114-4100

S. Ahrends

Oak Ridge Operations office

U.S. Department of Energy

P.0. Box E

Oak Ridge, TN 37830

J. LaGrone (Manager)

Oak Ridge Operations office

U.S. Department of Energy

P.0. Box E

0ak Ridge, TN 37830

D. E. Large

Oak Ridge Operations Office

U.S. Department of Energy

P.0. BOX E

Oak Ridge, TN 37830

C. L. Matthews (Chief, Fission Reactor Branch)

0 ak Ridge Operations office

U.S. Department of Energy

P.0. Box E

Oak Ridge; TN 37830
No. of

Copies

R. Philippone

0 ak Ridge Operations Office

U.S. Department of Energy

P.0. Box E

Oak Ridge, TN 37830

L. Lanni

San Francisco Operations office

U.S. Department of Energy

1333 Broadway, Hells Fargo Building

Oakl and, CA 94612

W. J. Brumley

Savannah River Operations office

U.S. Department of Energy

P.0. Box A

Aiken, SC 29801

G. K. Dertel

Savannah River Operations office

U.S. Department of Energy

P.0. Box A

Aiken, SC 29801

H. B. Saucier

Savannah River Operations office

U.S. Department of Energy P.0. Box A

Aiken, SC 29801

W. Hannum West Valley Project Office U.S. Department of Energy P.0. Box 191

West Valley, NY 14171-0191

Technical Library

West Valley Project Office

U.S. Department of Energy

P.0. Box 191

West Valley, NY 14171-0191

27

Office of Technical and Scientific Information P.0. Box 62

0ak Ridge, TN 37830

DOE CONTRACTORS

L. Burris

Argonne National

Laboratory

9700 South Cass Avenue

Argonne, IL 60439 
No. of

Copies

J. H. Kittel, OTD, Bldg. 208

Argonne National Laboratory

9700 South Cass Avenue Argonne, IL 60439

M. J. Steindler, CMT-205 Argonne National Laboratory

9700 South Cass Avenue

Argonne, IL 60439

10 Jo Ellen Balon

Office of Nuclear Waste Isolation

Battelle Menorial

Institute

505 King Avenue

Columbus, $\mathrm{OH} 43201$

F. Djahanguiri

Office of Nuclear Waste Isolation

Battelle Memorial

Institute

$505 \mathrm{King}$ Avenue

Columbus, $\mathrm{OH} 43201$

B. Rawles

Office of Nuclear Waste Isolation

Battelle Memorial

Institute

505 King Avenue

Columbus, $\mathrm{OH} 43201$

R. A. Robinson

Office of Crystalline Repository Development

Battelle Memorial Institute

$505 \mathrm{King}$ Avenue

Columbus, $\mathrm{OH} 43201$

P. Colombo, Bldg. 701

Brookhaven National

Laboratory

Upton, NY 11973

P. W. Levy

Brookhaven National

Laboratory

Upton, NY 11973

NWM Library

ATTN: S.G. Lane, Bldg. 830

Brookhaven National Laboratory

Upton, NY 11973
No. of

Copies

G. R. Vineyard

Brookhaven National

Laboratory

Upton, NY 11973

G. F. Curtin

Atomic Energy Division

E. I. Du Pont de Nemours \& Co., Inc.

6600 Montchanin Building

Wilmington, DE 19898

Document Custodian

Atomic Energy Division

E. I. Du Pont de Nemours \& Co., Inc.

6600 Montchanin Building

wilmington, DE 19898

R. J. Carpenedo

EG\&G Idaho, Inc.

P.0. Box 1625

Idaho Falls, ID 83415

E. A. Jennrich

EG\&G Idaho, Inc.

P.0. Box 1625

Idaho Falls, ID 83415

D. L. Uhl, WCB E-2

EG\&G Idaho, Inc.

P.0. Box 1625

Idaho Falls, ID 83415

C. R. Schuller

Battelle

Human Affairs Research

Centers

4000 NE 41 st Street

Seattle, WA 98105

Library

ATTN: Barbara Keen

Battelle

Human Affairs Research Centers

4000 NE 41 st Street

Seattle, WA 98105

L. Borduin, MS 517

Los Al amos National

Laboratory

P.0. Box 1663

Los Alamos, NM 87545

D. M. Kerr (Director)

Los Alamos National

Laboratory

P.0. Box 1663

Los Alamos, NM 87545
No. of

Copies

2 Report Library, MS-P364

Los Alamos National Laboratory

P.0. Box 1663

Los Alamos, NM 87545

D. T. Oakley, MS 671

Los Alamos National

Laboratory

P.0. Box 1663

Los Alamos, NM 87545

B. Perkins, MS 495

Los Alamos National Laboratory

P.0. Box 1663

Los Alamos, NM 87545

P. A. Witherspoon

Lawrence Berkeley Laboratory

Earth Science Division

1 Cyclotron Road

Berkeley, CA 94720

R. E. Batzel (Director)

Lawrence Livermore

National Laboratory

University of California

P.0. Box 808

Livermore, CA 94550

J. Campbel1, L-207

Lawrence Livermore

National Laboratory

University of California

P.0. Box 808

Livermore, CA 94550

R. Eddy, L-389

Lawrence Livernore

National Laboratory

University of California

P.0. Box 808

Livermore, CA 94550

L. D. Ramspott, L-204

Lawrence Livermore

National Laboratory

University of California

P.0. Box 808

Livermore, CA 94550

Technical Information Department, L-53

Lawrence Livermore

National Laboratory

University of California

P.0. Box 808

Livermore, CA 94550 
No, of

Copies

J. S. Baldwin

Oak Ridge National Laboratory

P.0. Box $X$

Oak Ridge, TN 37831

J. 0. Blomeke

Oak Ridge National Laboratory

P.0. Box $X$

Oak Ridge, TN 37831

L. R. Dole

Oak Ridge National Laboratory

P.0. Box X

Oak Ridge, TN 37831

M. Feldman

Oak Ridge National Laboratory

P.0. Box X

Oak Ridge, TN 37831

H. W. Godbee

Oak Ridge National Laboratory

P.0. Box $X$

Oak Ridge, TN 37831

L. J. Mezga, Bidg. $1505 / 106$

0ak Ridge National Laboratory

P.0. Box $X$

Oak Ridge, TN 37831

H. Postma

Oak Ridge National Laboratory

P.0. Box $X$

0ak Ridge, TN 37831

T. H. Row, Bldg. $4500-\mathrm{N}, \mathrm{C}-36$ Oak Ridge National Laboratory

P.D. Box X

Oak Ridge, TN 37831

J. G. Stradley, Bldg. 7601, MS 002

Oak Ridge National Laboratory

P.0. Box X

Oak Ridge, TN 37831
No. of

Copies

D. B. Trauger

Oak Ridge National Laboratory

P.0. Box X

Oak Ridge, TN 37831

Central Research Library, Acquisitions Section, Room I-103, Bldg. $4500 \mathrm{~N}$

Oak Ridge National Laboratory

P.0. Box X

Oak Ridge, TN 37831

G. W. Meyers

Atomics International Division

Rockwell International

8900 De Soto Avenue

Canoga Park, CA 91304

E. V. Ramirez

Atomics International Division

Rockwell International

8900 De Soto Avenue

Canoga Park, CA 91304

L. R. Crisler

Rocky Flats Plant

Rockwell International Corporation

P.0. Box 464

Golden, CD 80401

K. V. Gilbert

Rocky Flats Plant

Rockwell International Corporation

P.0. Box 464

Golden, CO 80401

P. G. Hagen

Rocky Flats Plant

Rockwell International Corporation

P.0. Box 464

Golden, CO 80401

R. H. Karlsson

Rocky Flats Plant

Rockwell International Corporation

P.0. Box 464

Golden, CO 80401
No. of

Copies

L. Smith

Rocky Flats Plant

Rockwell International

Corporation

P. 0 . Box 464

Golden, CO 80401

D. L. Ziegler Rocky Flats Plant Rockwell International

Corporation

P.0. Box 464

Golden, CO 80401

M. E. Spaeth

Science Applications, Inc. 2769 South Highland

Las Vegas, NV 89109

4 D. R. Anderson

Sandia National Laboratories

P.0. Box 5800

Albuquerque, NM 87185

G. C. Dacey

Sandia National Laboratories

P.0. Box 5800

Albuquerque, NM 87185

T. D. Hunter, NNWSI-6310

Sandia National

Laboratories

P.0. Box 5800

Albuquerque, NM

87185

R. M. Jefferson

Sandia National Laboratories

P.0. Box 5800

Aibuquerque, NM

87185

R. Lincoln

Sandia National Laboratories

P.0. Box 5800

Albuquerque, NM 87185

Technical Library

Sandia National

Laboratories

P.0. Box 5800

Albuquerque, NM 87185

R. W. Lynch

Sandia National

Laboratories

P.0. Box 5800

Albuquerque, NM 87185 
No. of

Corpies

2 L. Sheppard, Div. 9734

Sandia National

Laboratories

P.0. Box 5800

Albuquerque, NM 87185

D. M. Talbert

Sandia National

Laboratories

P.0. Box 5800

Albuquerque, NM 87185

W. D. Weart, Dept. 6330

Sandia National

Laboratories

P.0. Box 5800

Albuquerque, NM 87185

E. L. Albenesius, Research Manager

Savannah River Laboratory

E. I. du Pont - SRP

Aiken, SC 29808

J. L. Crandall

Savannah River Laboratory

E. I. du Pont - SRP

Aiken, SC 29808

2 Document Control Group TIS, Bldg. 773A, Room A-0281

Savannah River Laboratory

E. I. du Pont - SRP

Aiken, SC 29808

2 E. J. Hennelly, 773-19A

Savannah River Laboratory

E. I. du Pont - SRP

Aiken, SC 29808

W. R. Stevens, III

Savannah River Laboratory

E. I. du Pont - SRP

Aiken, SC 29808

S. Mirshak

Savannah River Laboratory

E. I. du Pont - SRP

Aiken, SC 29808

M. J. Plodinec

Savannah River Laboratory

E. I. du Pont - SRP

Aiken, SC 29808

H. J. Groh

Savannah River Plant

Aiken, SC 29808
No. of

Copies

R. Maher

Savannah River Plant

Aiken, SC 29808

J. B. Mellen

Savannah River Plant

Aiken, SC 29808

J. H. Saling, Manager, HLW/SFP

Westinghouse Electric Corp.

Waste Technology, Services Division

P.0. Box 10864

Pittsburgh, PA 15236

2 R. A. Brown

Westinghouse Idaho Nuclear Co., Inc.

P.0. Box 4000

Idaho Falls, ID 83401

M. P. Johnson

Westinghouse Idaho Nuclear Co., Inc.

P.0. Box 4000

Idaho Falls, I0 83401

B. R. Wheeler

Westinghouse Idaho Nuclear Co.., Inc.

P.0. Box 4000

Idaho Falls, ID 83401

A. Metry

Weston

2301 Research Boulevard

Rockville, MD 20850

2 J. F. Strahl

Weston

2301 Research goulevard

Rockville, MD 20850

J. L. Knabenschuh

west Valley Nuclear

Services Co., Inc.

P.0. Box 191

West Valley, NY 14171-0191

E. Meyers

West Valley Nuclear

Services Co., Inc.

P.0. Box 191

West Valley, NY 14171-0191
No. of

Copies

\section{OTHER AGENCIES}

2 R. Balent

Electric Power Research Institute

P.0. Box 10412

3412 Hillview Avenue

Palo Alto, CA 94304

3 C. Braun

Electric Power Research Institute

P.0. Box 10412

3412 Hillvien Avenue

Palo Alto, CA 94304

F. Rahn

Electric Power Research Institute

P.0. Box 10412

3412 Hillview Avenue

Palo Alto, CA 94304

R. F. Williams

Electric Power Research Institute

P.0. 80x 10412

3412 Hillview Avenue

Palo Alto, CA 94304

Library

General Atomics

Technologies, Inc.

P.0. Box 81608

San Diego, CA 92138

R. N. Drake

General Atomics Technologies, Inc.

P.0. Box 81608

San Diego, CA 92138

H. W. Paige

International Energy Associates, Limited

600 New Hampshire

Avenue, $\mathrm{NW}$

Washington, DC 20037

J. C. Young

International Energy Associates, Limited

600 New Hampshire

Avenue, NW

Washington, DC 20037

E. R. Johnson

E. R. Johnson Associates, Inc.

11702 Bowman Green Drive

Reston, VA 22090 
No. of Copies

J. A. McBride

E. R. Johnson Associates, Inc.

11702 Bowman Green Drive

Reston, VA 22090

G. R. Heath

Oregon State University

School of Oceanography

Corvallis, OR 97331

M. Eisenbud

New York University

Medical Center

Laboratory for

Environmental Studies

Box 817

Tuxedo, NY 10987

FEDERAL AGENCIES

A. Dravo

House Interior and Insular Compittee

1327 Longworth Office Bldg.

Washington, DC 20515

J. Duggan

U.S. House of Representatives

Subcommittee on Energy Research and Production

B-374 Rayburn House Office Building

Washington, DC 20515

J. Freiwald

U.S. House of Representatives

Subcomnittee on Energy Research and Production

B-374 Rayburn House Office Building

Washington, DC 20515

R. Ketchan

U.S. House of Representatives

Subcomittee on Energy Research and Production

B-374 Rayburn House Office Building

Washington, DC 20515
No. of

Copies

L. Ventre

U.S. House of

Representatives

Subcomittee on Energy

Research and Production

B-374 Rayburn House Office Building

Washington, DC 20515

R. Civiak

Congressional Research Services/SPRO

Library of Congress

Washington, DC 20540

2 J. P. Boright

O.S. Embassy - Paris, France

Science Counselor

APO New York, NY 09777

G. Helfrich

US Embassy - Tokyo, Japan

Science Counselor

APO San Francisco, CA 96503

J. Bloom

U.S. Embassy - London, UK

Science Counselor

FPO New York, NY 09510

D. M. Marshall

U.S. Mission to EC Brussels

Energy and Resources 0 fficer

APO New York, NY 09667

A. H. Reynolds

U.S. Mission to OECD

c/o US Embassy - Paris, France

APO New York, NY 09777

W. Grieder, A-106

Environmental Protection Agency

Office of International Activities

Washington, DC 20460

F. L. Galpin, MC ANR

Environmental Protection Agency

Waste Management Standards Branch

Criteria and Standards

Division

Washington, DC 20460
No. of

Copies

W. F. Holcomb, MC ANR-460

Environmental Protection Agency

Office of Radiation

Programs

401-M Street, S.W.

Washington, DC 20460

G. L. Sjoblom, MC ANR -460

Environmental Protection

Agency

Office of Radiation

Programs

401-M Street, S.W.

Washington, DC 20460

T. Tolson, MC ANR -458

Environmental Protection Agency

Office of Radiation Programs

401-M Street, S.W.

Washington, DC 20460

P. Myers

National Academy of Sciences

Board on Radioactive Waste Management

Room JH-826

2101 Constitution

Avenue, Nw

Washington, DC 20418

D. M. Rohrer, MS-359-EW/W

U.S. Nuclear Regulatory Commission

Office of Inspection and Enforcement

Washington, DC 20555

K. D. Burke, MS 406-EWS

U.S. Nuclear Regulatory Commission

Office of International Programs

Washington, DC 20555

4 M. R. Peterson, MS 414A-EWS

U.S. Nuclear Regulatory Commission

office of International Programs

Washington, DC 20555 
No. of

Copies

D. Al exander, MS 1130-SS

U.S. Nucl ear Regul atory Commission

Office of Nuclear Regulatory Research

Division of Health, Siting, and Waste Management

Washington, DC 20555

F. Arsenault, MS 1130-SS

U.S. Nuclear Regulatory Commission

Office of Nuclear Regulatory Research

Division of Health, Siting, and Waste Management

Washington, DC 20555

Dr. Jose L. M. Cortez, MS 1130 -SS

U.S. Nuclear Regulatory Commission

Office of Nuclear Regulatory Research

Division of Health, Siting, and Waste Management

Washington, DC 20555

3 J. J. Davis, MS 1130-SS

U.S. Nuclear Regulatory Commission

Office of Nuclear Regulatory Research

Division of Health, Siting, and Waste Management

Washington, DC 20555

Docket Control Center, MS 623-SS

U.S. Nuclear Regulatory Cormission

Office of Nuclear Regulatory Research

Division of Health, Siting, and Waste Management.

Washington, DC 20555
No. of

Copies

Reference Library ATTN: M Conyngham, MS 160

U.S. Nuclear Regulatory Conmission

Office of Nuclear Regulatory Research

Division of Health, Siting, and Waste Management

Washington, DC 20555

J. C. MCKinley, MS H-1016

U.S. Nuclear Regulatory Commission

Advisory Committee on Reactor Safeguards

Washington, DC 20555

L. Wirfs, MS 881-SS

U.S. Nuclear Regulatory Commission

Advisory Conmittee on Reactor Saf eguards

Washington, DC 20555

R. D. Bennett

U.S. Department of Interior

Waterways Experiment Station

P.0. Box 631

Vicksburg, MS 39180

P. Brush

U.S. Department of State

c/o U.S. Mission to IAEA/Vienna

Counselor for Atomic Energy Af fairs

Washington, DC 20520

A. Sessoms

U.S. Department of State OES/NET/EST

Room 7828

22nd and C Streets, NW

Washington, DC 20520

S. Patterson

U.S. Department of State OES/SAT

22nd and $C$ Streets, NW

Washington, $O C 20520$

J. Williamson

U.S. Department of State OES/ENH

Room 7820, Mail Stop 27

22 nd and $C$ Streets, NW

washington, OC 20520
No. of

Copies

J. B. Robertson, MS 410

U.S. Geological Survey

Office of Radiohydrology

Reston, VA 22092

J. M. Clevel and

U.S. Geological Survey

Denver Federal Center

MS 412

Denver, CO 80225

STATE AGENCY

L. Slosky

Office of the Governor

Assistant to the Governor for Science Technology

127 State Capitol,

Room 127

Denver, CO 80222

FOREIGN AGENCIES

Mr. W. Drent

Eurochenic

B-2400 Mol

Belgium

Prof.-Dr. P. Delonghe

Laboratory of the

S.C.K./C.E.N.

Boeretang 200

8-2400 Mol

Belgium

2 T. E, Rumnery

Atomic Energy of Canada, Ltd.

Whiteshell Nuclear Research Establishment

Waste Management Division Pinawa, Manitoba, ROE ILO Canada

Dr. Serge Orlowski

Commission of the European Communities (CEC)

200 , rue de la Loi

B-1049 Brussels

Belgium

J. Gaunt

British Embassy

3100 Massachusetts

Avenue, NH

Washington, DC 20008 
No. of

Copies

2 J. M. Lavie

General Manager

Agence Nationale pour

la Gestion des Dechets Radioactifs

Commissariat a 1 'Energie Atomique

$31-33$, rue de la

Federation

75752 Paris, Cedex 15

France

2 J. Lefevre

General Manager

Agence Nationale pour la Gestion des Dechets Radioactifs

Comnissariat a l'Énergie Atomique

31-33, rue de la

Federation

75752 Paris, Cedex 15

France

2 P. Jourde

International Programs

Nuclear Waste Management

Fontenay-aux-Roses Centre d'Etudes Nucléaires (CEN-F aR)

B.P. 6

F-92260 Fontenay-aux-Roses

France

G. Baudin

International Programs

Nuclear Waste Management

Fontęnay-aux-Roses Centre d'Etudes Nucleaires (CEN-FaR)

B.P. 6

F-92260 Fontenay-aux-Roses

France

2 Dr. Rolf-Peter Rand

Bundesministerium für

Forschung und Technologie

Heinemannstrasse 2

D-5300 Bonn-Bad Godesberg

Postfach $20 \quad 07 \quad 06$

Federal Republic of Germany
No. of

Copies

Dr. Klaus Kuhn

Gesellschaft für Strahlen-und Umwelt forschung mbH Munchen/Institut für Tieflagerung (GSF/IfT)

Theodor-Heuss-Strasse 4

D-3300 Braunschweig

Federal Republic of Germany

Dr. Reinhard H. Kroebel

Kernforschungszent rum $\mathrm{Karl}$ sruhe GmbH

Postfach 3640

D-7500 Karlsruhe 1

Federal Republic of Germany

A. Hagen

International Atomic Energy Agency

Waste Management Section

Division of Nuclear Fuel Cycle

Wagramerstrasse 5,

P.0. Box 100

A-1400, Vienna Austria

K. T. Thomas

International Atomic Energy Agency

Waste Management Section

Division of Nuclear Fuel Cycle

Wagramerstrasse 5 ,

P.0. Box 100

A-1400, Vienna Austria

D. E. Saire, Scientific Secretary

International Atomic Energy Agency

Waste Management Section

Division of Nuclear Fuel Cycle

Wagramerstrasse 5 ,

P.O. Box 100

A-1400, Vienna Austria

G. Delcoigne

International Atomic Energy Agency

Division of Public Information

Wagramerstrasse 5 ,

P. 0. Box 100

A-1400, Vienna Austria
No. of

Copies

2 K. Uematsu, Director

Power Reactor and Nuclear Fuel Development Corporation (PNC)

Sankaido Building

1-9-13 Akasaka

Minato-ku, Tokyo 107

Japan

S. Godwin

OECD Nuclear Energy Agency

38 Boulevard Suchet

F-75016 Paris

France

J.-P. Olivier

OECD Nuclear Energy Agency

38 Boulevard Suchet

F-75016 Paris

France

H. Shapar

OECD Nuclear Energy Agency

38 Boulevard Suchet

F-75016 Paris

France

G. Rundquist

Naenmden foer Hantering av Atvaent Kaernbraensle

Kunysgat an 38

S-111 56 Stockholm

Sweden

2 C. Thegerstrom

Svensk

Kärnbränsleförsörjning $A B$ (SKBF)

BOX 5864

S-102 48 Stockholm

Sweden

2 Dr. Rudolph Rometsch

President

National Cooperative for the Storage of

Radioactive Waste (NAGRA/CEDRA)

Parkstrasse 23

CH-5401 Baden

Switzerl and

2 Dr. Frank S. Feates

Nuclear Waste Management Division

Department of the Environment

2 Marsham Street London SWIP 3EB

United Kingdom 
No. of

Copies

Dr. R. H. Flowers UKAEA Atomic Energy Research Establishment, AERE

Harwell, Didcot

OXON OXI1 ORQ

United Kingdom

\section{ONSITE}

13 DOE Richland Operations Office

T. A. Bauman

M. Dayani (6)

A. G. Fremling

R. A. Holten

P. Brimhall

H. E. Ransom

F. R. Standerfer

J. D. White
No. of

Copies

56 Pacific Northwest Laboratory

W. F. Bonner

E. C. Corley

D. B. Cearlock

J. M. Davidson (10)

L. R. Dodd

K. M. Harmon (20)

A. B. Johnson, Jr.

R. J. Kofoed

L. T. Lakey

I. W. Leigh

R. C. Liikala

J. L. MCEl roy

J. E. Mendel

D. R. Oden

A. M. Platt

D. E. Robertson, Jr.

J. V. Robinson

K. J. Schneider

R. J. Serne

H. L. Templeton

L. D. Willians

Publishing Coordination (2)

Technical Information (5)
No. of

Copies

5 Rockwell-Hanford

C. L. Brown

P. G. Lorenzini

J. K. Patterson

M. J. Smith

V. V. Ufkes, Technical Information Center

UNC Nuclear Industries

W. J. Kyriazis

3 Westinghouse Hanford Corporation

A. G. Blasewitz

E. A. Evans

S. J. Mayhan 